\title{
Investigation of the Structure and Dynamics
}

\section{of Multiferroic Systems by Inelastic Neutron}

\section{Scattering and Complementary Methods}

\author{
Dissertation \\ for the award of the degree \\ "Doctor rerum naturalium" (Dr.rer.nat.) \\ of the Georg-August-Universität Göttingen \\ within the doctoral program Chemistry \\ of the Georg-August University School of Science (GAUSS)
}

submitted by

Fabian Ziegler

from Kassel

Göttingen, 2018 
Thesis Committee

Prof. Dr. Götz Eckold, Institut für Physikalische Chemie

Prof. Dr. Dietmar Stalke, Institut für Anorganische Chemie

\section{Members of the Examination Board}

Reviewer: $\quad$ Prof. Dr. Götz Eckold, Institut für Physikalische Chemie

Second Reviewer: $\quad$ Prof. Dr. Dietmar Stalke, Institut für Anorganische Chemie

Further members of the Examination Board:

Prof. Dr. Jörg Behler, Institut für Physikalische Chemie Prof. Dr. Martin Suhm, Institut für Physikalische Chemie Prof. Dr. Franc Meyer, Institut für Anorganische Chemie Prof. Dr. Burkhard Geil, Institut für Physikalische Chemie 


\section{Table of content}

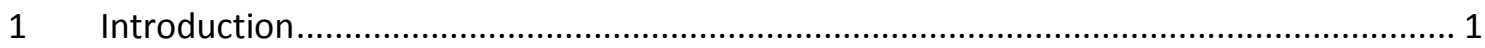

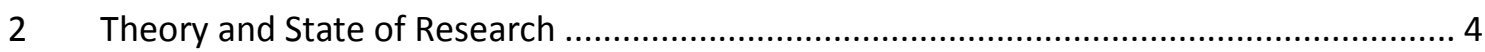

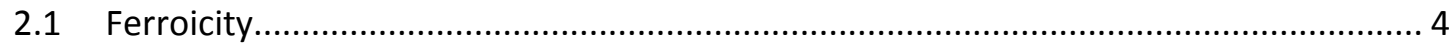

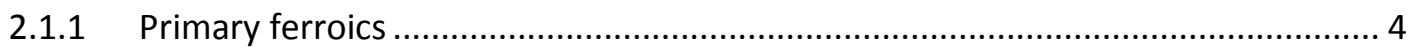

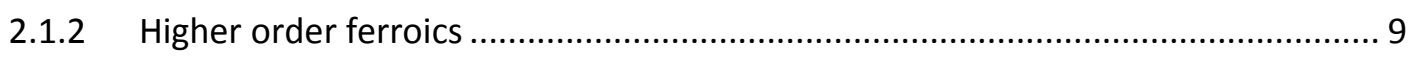

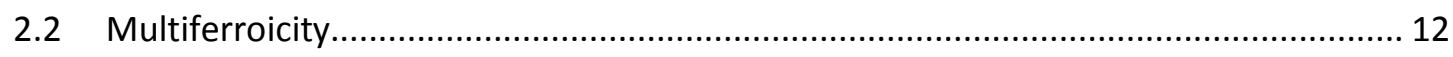

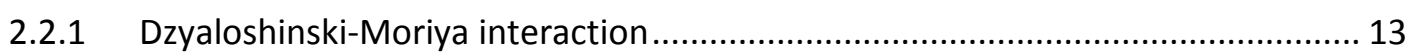

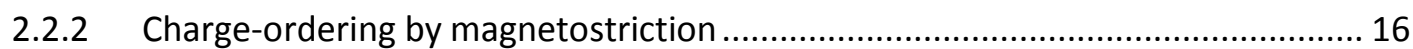

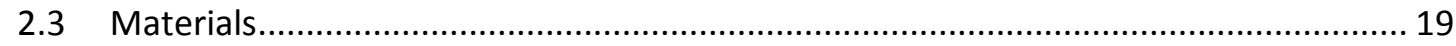

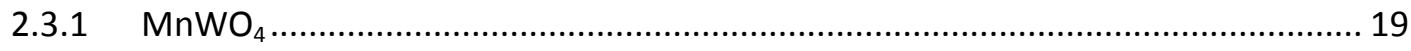

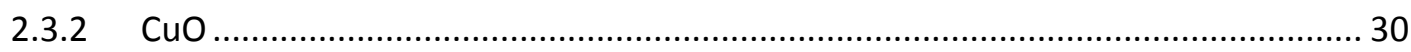

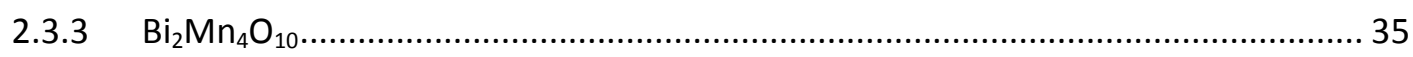

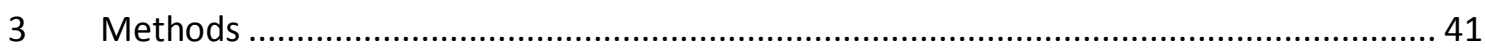

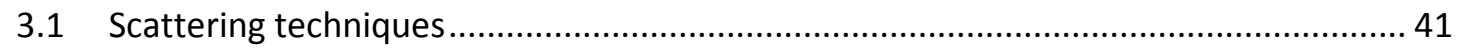

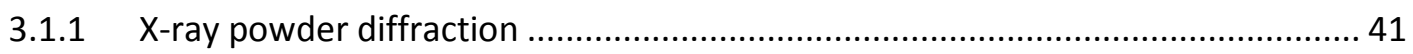

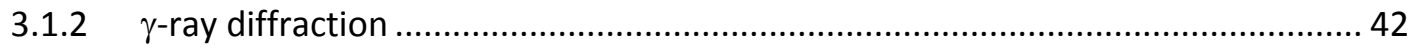

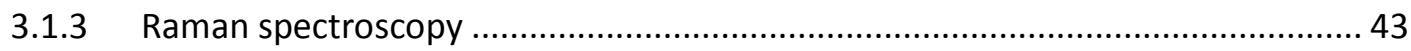

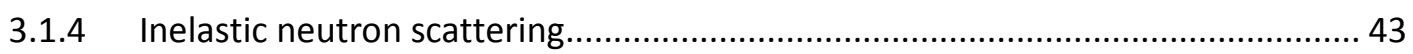

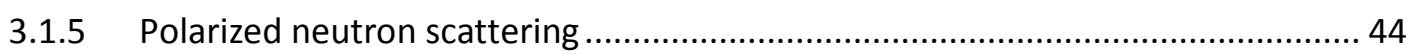

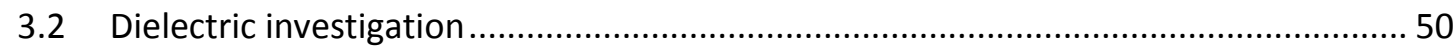

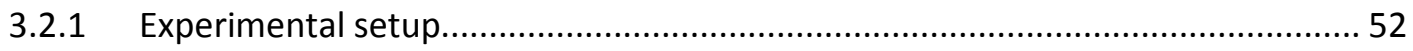

3.2.2 Circuit diagrams and data evaluation.............................................................. 53

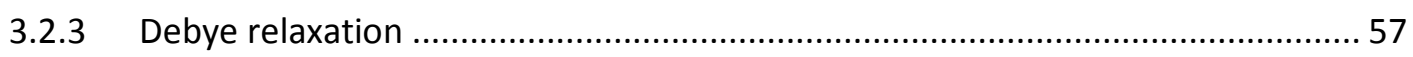

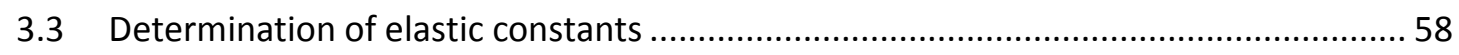

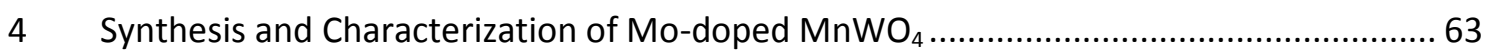

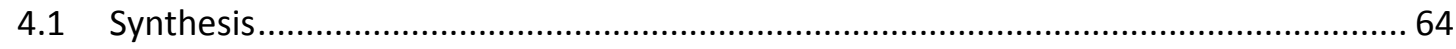

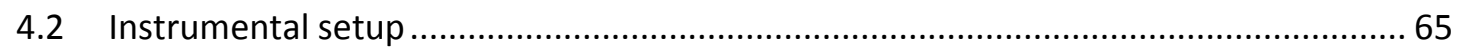




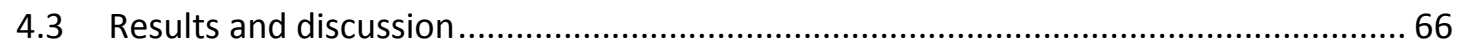

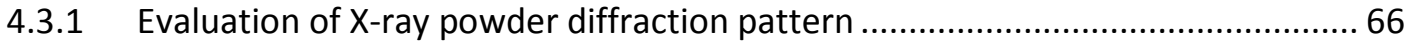

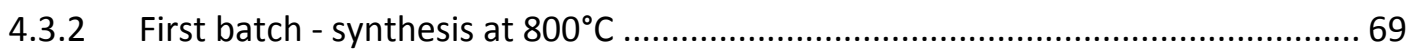

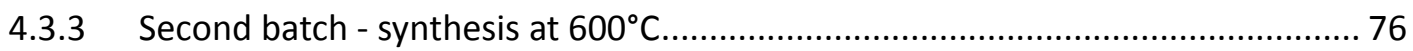

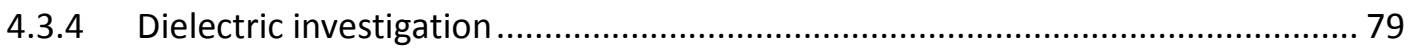

4.3.5 Magnetic susceptibility of selected samples ................................................... 86

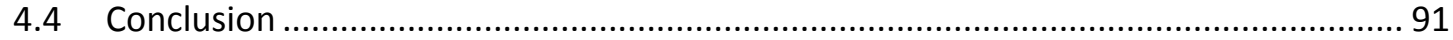

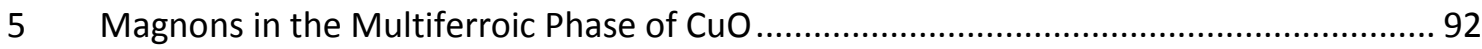

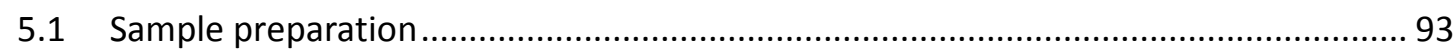

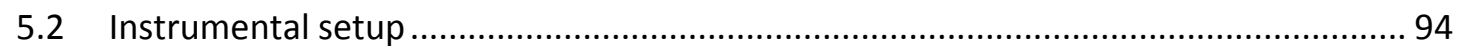

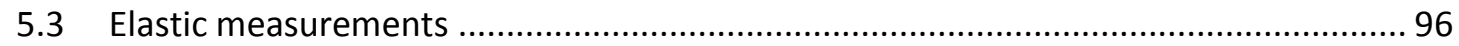

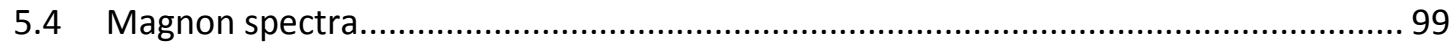

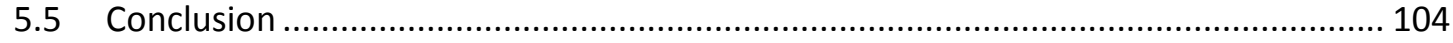

$6 \quad$ Dielectric and Mechanical Investigation of BMO ....................................................... 105

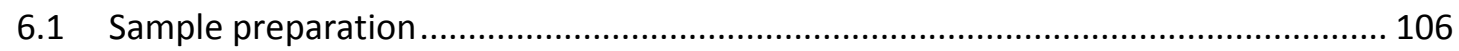

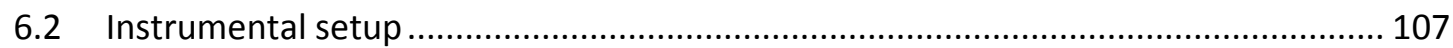

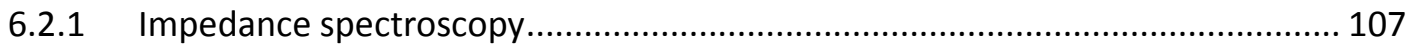

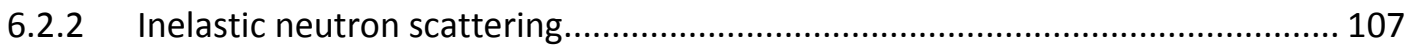

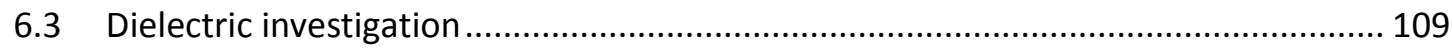

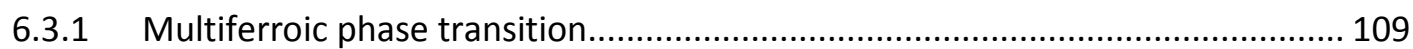

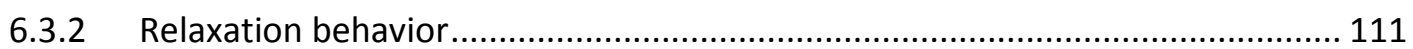

6.3.3 Amendment after submission of the thesis ................................................... 116

6.4 Determination of elastic constants .................................................................... 117

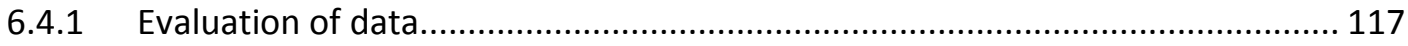

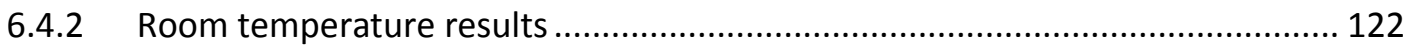

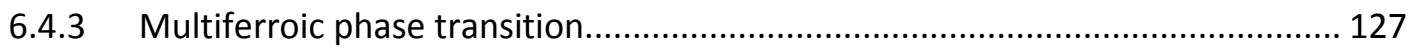

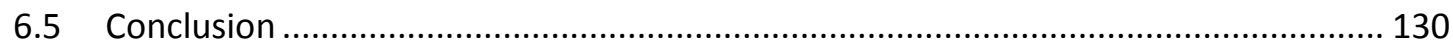

7 Summary. 


\section{Introduction}

With the beginning of modern science, mankind started to formulate complex theories with the aim to understand nature. The ultimate goal for generations of scientists is the search for a "theory to describe everything". The closest equivalent in modern day physics that could satisfy this urge is the unified field theory that got its first impetus with the description of light as an electromagnetic wave in the middle of the $19^{\text {th }}$ century by Maxwell [1]. The combination of magnetic and electric properties in solid materials, on the other hand, is not straightforward and proves to be a continuous endeavor. It took nearly hundred years from the formulation of Maxwell's equations until solid state physicists discovered the linear magnetoelectric (ME) effect [2], which initiated a whole new field of research.

At that time, it was well known that the application of an electric field $\boldsymbol{E}$ on a material induces an electric polarization $\boldsymbol{P}$. The same is true for the magnetic field $\boldsymbol{H}$ and the magnetization $\boldsymbol{M}$. The ME effect, on the other hand, allows the induction of polarization or magnetization by the respective opponent field [3]. Astrov presented the experimental evidence for the ME effect 1960 in the Soviet Union as he measured a non-vanishing magnetization in $\mathrm{Cr}_{2} \mathrm{O}_{3}$ while applying an electric field [2]. This effect was rather weak why further effort was put into the search for substances with stronger ME effect. One particular kind of material caught the interest. Ferromagnets with spontaneous magnetization $\boldsymbol{M}_{(\mathrm{s})}$ and ferroelectrics with spontaneous polarization $\boldsymbol{P}_{(\mathrm{s})}$ were well known at that time, but materials with both types of ordering in a single phase emerged as a new field of research [4]. The hope was that this static coexistence of electric and magnetic properties could lead to a strong ME effect.

More of these "ferroelectromagnets", as they were called back then, were discovered [5] but after a while the interest in this topic faded and it took a few decades until it was revived in the year 1994 by Schmid [6]. He pointed out the importance of a third kind of ordering, called ferroelasticity, and therefore coined the term "multiferroic". Ferroelasticity was first introduced by Aizu [7] and describes the occurrence of spontaneous strain $\varepsilon_{(s)}$. Schmid also provided a reference point in terms of symmetry with a list of point groups and their allowed ferroic properties [6], based on Aizu's work. This was complemented by Hill with an elaboration about the problems of designing multiferroics from a chemical point of view [8]. From this point on, the search for new multiferroics and the understanding of the intrinsic mechanisms became a "hot topic" in solid state research.

The first multiferroics that were discovered showed ferroelectricity and ferromagnetism (in most cases it is rather ferri- or antiferromagnetism) in a single phase, but both phenomena had different origins and were not directly coupled [9]. They could not fulfill the initial hope to find 
materials with a strong ME effect and were later labeled "type-I" multiferroics [10]. The great breakthrough came in 2003 when Kimura presented an experiment on $\mathrm{TbMnO}_{3}$ where it is possible to alter the magnitude of both, the dielectric constant and the electric polarization, by applying a magnetic field [11]. This is possible because the ferroelectricity is directly caused by magnetic ordering [12-14] and therefore leading to the desired magnetoelectric coupling. These multiferroics are called "type-II" [10] and became a new focus of research.

The expectation that all type-II multiferroics exhibit a strong ME effect is, however, not satisfied. In some cases, this is true but it is rather a coincidence than a rule [3]. At this point it is important to distinguish two terms. If, for example, the polarization of a material can be switched off [15] or tilted [11] when a magnetic field is applied, this is sometimes referred to as "magnetoelectric effect" $[11,16]$, but it is something different than the aforementioned "linear magnetoelectric effect". While research on the latter nowadays rather focusses on strain mediated multiple-phase-systems and nanocomposites [16], "multiferroics" became a topic on its own due to their rich diversity and wide range of physical discoveries.

The magnetic ordering in type-II multiferroics follows the same mechanisms like in any common non-ferroelectric (anti-)ferromagnet, but the cause for electric ordering is completely different compared to conventional ferroelectrics. In fact, there is not the one "multiferroic coupling" but rather a variety of different mechanisms were identified so far [10]. Comparing a range of different multiferroics, there are a few common properties that can be observed in many materials, but there are also members of the same substance class that exhibit distinct differences. In the end, each multiferroic deserves its own attention and has to be treated as unique.

For this reason, current research on multiferroics focusses on three tasks. First, there is the search for new multiferroic materials. In fact, more and more classes of substances are found until today (one recent example: [17]). The "holy grail" would be a type-II multiferroic with a stable multiferroic phase at room temperature because this is the necessary condition for industrial applications, especially for wide spread consumer electronics. The second task is to characterize all relevant properties of each material. This is important for the understanding of the multiferroic coupling mechanisms and goes hand in hand with the third task: tailoring multiferroics according to own needs. This is usually done by substitution of an ion by another one to a certain degree in order to purposefully alter certain properties, for example to increase the phase transition temperature.

The research of this thesis engages with the latter two tasks and can be divided in three distinct parts, each focusing on one specific substance. Mo-doped $\mathrm{MnWO}_{4}$ is investigated In the first part, because it was reported that the rather low multiferroic transition temperature can be 
increased when tungsten atoms are substituted by molybdenum [18]. The system $\mathrm{MnW}_{1-x} \mathrm{Mo}_{x} \mathrm{O}_{4}$ exhibits a miscibility gap, which is not very well characterized, and there are contradicting results about the maximum amount of molybdenum that can be solved in $\mathrm{MnWO}_{4}$. Consequently, it is unclear how far the phase transition temperature can be increased. Samples with different amount of Mo-doping are therefore synthesized in order to characterize the miscibility gap and to determine the maximum transition temperature that is achievable.

The second part of this thesis focusses on $\mathrm{CuO}$ that has a similar magnetic structure like $\mathrm{MnWO}_{4}$, but exhibits a higher ferroelectric phase transition temperature than many other multiferroics [19]. Furthermore, an electromagnon was recently discovered in the multiferroic phase of $\mathrm{CuO}$ [20], which can be regarded as an elementary excitation of the multiferroic coupling. The characterization of this magnon can therefore help to better understand the underlying mechanism that causes multiferroicity in CuO. Currently, there are several publications about the determination of magnons by inelastic neutron scattering, but only in the non-multiferroic low-temperature phase. Therefore, magnon spectra are measured in the multiferroic phase with neutron polarization analysis in order to characterize the eigenvector of all measured magnons.

The third part of this thesis is about $\mathrm{Bi}_{2} \mathrm{Mn}_{4} \mathrm{O}_{10}$, which has a magnetic structure that differs significantly from $\mathrm{MnWO}_{4}$ and $\mathrm{CuO}$ and so does the mechanism that is believed to cause multiferroicity [21]. However, unlike for other members of the $\mathrm{R}_{2} \mathrm{Mn}_{4} \mathrm{O}_{10}$-family, there is no experimental evidence that $\mathrm{Bi}_{2} \mathrm{Mn}_{4} \mathrm{O}_{10}$ is multiferroic at all. Therefore, the dielectric permittivity is measured in the vicinity of the magnetic phase transition temperature in order to definitely answer the question whether $\mathrm{Bi}_{2} \mathrm{Mn}_{4} \mathrm{O}_{10}$ is a type-II multiferroic. The proposed multiferroic coupling mechanism of this substance class is based on magnetostriction and the mechanical properties are therefore expected to change in the multiferroic phase. In order to clarify if this mechanism can also be applied to $\mathrm{Bi}_{2} \mathrm{Mn}_{4} \mathrm{O}_{10}$, all elastic constants were determined in the multiferroic phase as well as at room temperature for comparison. 


\section{Theory and State of Research}

This chapter will provide an overview over the theoretical foundations necessary for the understanding of multiferroics as well as the current state of research. The basic concept of ferroics and their different types are explained in the first subchapter while mechanisms for multiferroic coupling are discussed in the second subchapter. In the last subchapter, all three investigated materials and their relevant properties are presented.

\subsection{Ferroicity}

Before the theoretical basics of multiferroics can be explained, it is necessary to clarify the meaning of ferroicity in itself. The first subchapter will focus on primary ferroics which include commonly known ordering phenomena like ferromagnetism, ferroelectricity and ferroelasticity but also the recently discovered ferrotoroidicity. These primary ferroics are responsible for the properties that sparked the interest in multiferroics. Most quantities that are experimentally determined in this thesis, however, fall in the category of higher order ferroics that will be discussed in the second subchapter.

\subsubsection{Primary ferroics}

Primary ferroics are characterized by a contribution to the total free energy $F$ described by a macroscopic order parameter $\boldsymbol{O}$ and an external field $\boldsymbol{A}$ as [22]:

$$
F_{\text {ferroic }}=-\mathbf{O A}
$$

A further requirement is the existence of a high-temperature non-ferroic phase whose point group is in a group-subgroup-relationship with the low-temperature ferroic phase [23]. If the material experiences a transition into the ferroic phase, domains with different orientations of $\boldsymbol{O}$ will develop that are energetically degenerate. This degeneracy is lifted by applying $\boldsymbol{A}$ since the energy of each domain, according to equation (2-1), depends on the relative orientation of $\boldsymbol{O}$ and $\boldsymbol{A}$. It is therefore possible to switch all domains to one orientation of $\boldsymbol{O}$ with a strong enough $\boldsymbol{A}$ and thereby create a mono-domain crystal. This switching process can be reversed if an external field with another orientation is applied. In the one-dimensional case with two orientations (+ and -), this leads to a typical hysteretic behavior as shown in Figure 2-1.

There are four different types of primary ferroic order which transform according to different irreducible representations of the space-time-inversion group (see Table 2-1) [24]. This group consists of the four symmetry elements unity 1 , space-inversion $i$, time-inversion $1^{\prime}$ and their combination i' [25]. Ferroelasticity is totally symmetric and has the order parameter spontaneous strain $\varepsilon_{(s)}$ that can be altered by the external stress field $\sigma$. It is quite exceptional because both 
quantities are a second rank tensor while $\boldsymbol{O}$ and $\boldsymbol{A}$ of all other ferroic types are first rank vectors. Two further examples for $\boldsymbol{O}$ are the spontaneous polarization $\boldsymbol{P}_{(\mathrm{s})}$ and the spontaneous magnetization $\boldsymbol{M}_{(\mathrm{s})}$ which are invariant under time-inversion and space-inversion, respectively. This difference in symmetry is one of the reasons why it was believed that ferroelectricity and ferromagnetism were mutually exclusive in a single substance [8].

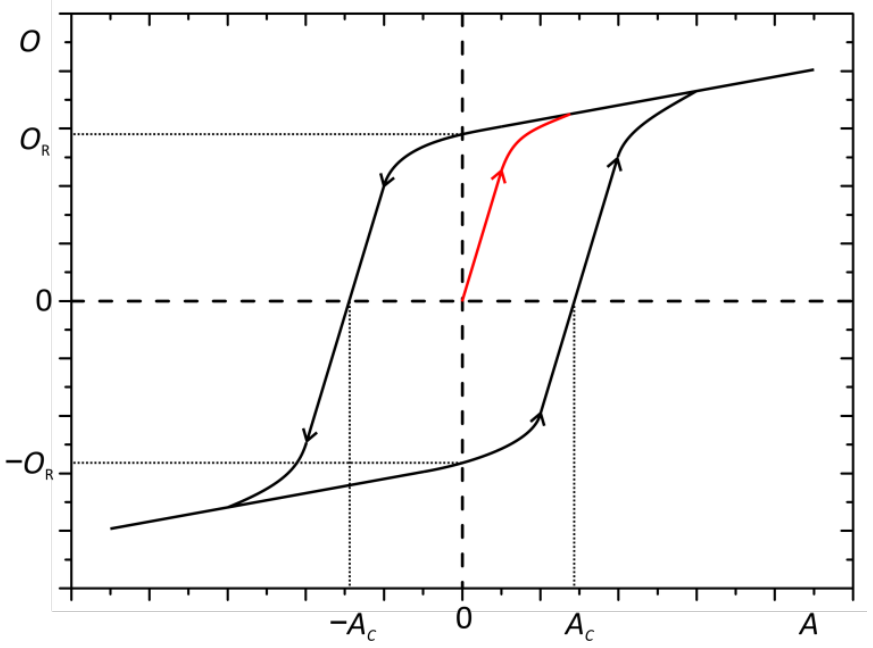

Figure 2-1: Schematic representation of a typical hysteresis loop of primary ferroics. The red curve describes the first poling. When the external field is turned off, a certain magnitude of the order parameter remains called remanence $\left(O_{R}\right)$. The external field that is needed to reverse the sign of the order parameter is called coercive field $A_{\mathrm{R}}$.

Table 2-1: Character table for the transformation under spatial inversion i, time inversion $1^{\prime}$ and the combination i'. Each of the four primary ferroics transforms according to one of the four irreducible representations. The order parameter $\boldsymbol{O}$ and the external field $\boldsymbol{A}$ of all four primary ferroics are listed $[24,25]$.

\begin{tabular}{|c|c|c|c|c|c|c|}
\hline $\mathbf{1}$ & $\mathbf{i}$ & $\mathbf{1}^{\prime}$ & $\mathbf{i}^{\prime}$ & type of order & $\boldsymbol{O}$ & $\boldsymbol{A}$ \\
\hline 1 & 1 & 1 & 1 & ferroelasticity & $\boldsymbol{\varepsilon}_{(\mathrm{s})}$ & $\boldsymbol{\sigma}$ \\
\hline 1 & -1 & 1 & -1 & ferroelectricity & $\boldsymbol{P}_{(\mathrm{s})}$ & $\boldsymbol{E}$ \\
\hline 1 & 1 & -1 & -1 & ferromagnetism & $\boldsymbol{M}_{(\mathrm{s})}$ & $\boldsymbol{H}$ \\
\hline 1 & -1 & -1 & 1 & ferrotoroidicity & $\boldsymbol{T}_{(\mathrm{s})}$ & $\boldsymbol{G}$ \\
\hline
\end{tabular}

After this general introduction, the following subchapters will describe some unique characteristics of primary ferroics that are relevant for this thesis.

\subsubsection{Ferromagnetism and antiferromagnetism}

The most common primary ferroic is ferromagnetism because it is present at room temperature in iron which is not only the second most abundant element on earth, but also lends its Latin name for the whole concept of ferroicity. The spontaneous magnetization $\boldsymbol{M}_{(\mathrm{s})}$ is explained on a microscopic level by the alignment of spins of unpaired electrons. This is driven by exchange 
interactions between two Spins $S_{\mathrm{i}}$ and $S_{\mathrm{j}}$ on neighboring atoms. The contribution to the total free energy can be described as [26]:

$$
F_{\mathrm{ex}}=J_{i j} \boldsymbol{S}_{\mathrm{i}} \cdot \boldsymbol{S}_{\mathrm{j}}
$$

If the exchange integral is $J_{i j}<0$, only a parallel alignment of spins can lower the free energy. A positive $J_{\mathrm{ij}}$, on the other hand, would favor an antiparallel ordering (please compare Figure 2-3 (a) and (b)). This latter phenomenon is called antiferromagnetism and is often observed in ionic substances were the magnetic metal atoms are separated by a diamagnetic ion like oxygen or halogens. The interaction between two spins is in this case mediated via the diamagnetic ion and therefore it is called "superexchange". In fact, this interaction has a different physical origin than direct exchange and can be described by the quantum mechanical approach of Anderson [26]. Since antiferromagnets are usually insulators, they have localized orbitals and are treated according to ligand field theory while classical ferromagnets are usually conducting metals. The localized $d$-orbitals of one metal ion overlap with the $p$-orbitals of surrounding diamagnetic ions and therefore also with the $d$-orbitals of the neigboring metal ions. In the unperturbed ground state of a metal with $d^{1}$-electron configuration, for example, there would be one unpaired electron per metal atom. Perturbation theory allows this ground state to be mixed with excited states where the unpaired electron of one metal ion occupies the orbital of an unpaired electron of the neighboring metal ion (see Figure 2-2). This "hopping" stabilizes the ground state, but it is only possible when both electrons have opposite spin because they would otherwise violate the Pauli exclusion principle. This leads to an antiferromagnetic ordering.

Despite the difference in its nature, superexchange interaction can also be described by equation (2-2). Only, the physical meaning of $J$ is different compared to its exchange interaction counterpart. Now it might be important to point out a caveat. It is true that exchange interaction always favors a parallel alignment and superexchange always favors an antiparallel alignment, but this does not mean that there is only superexchange in antiferromagnets. In fact, both interactions

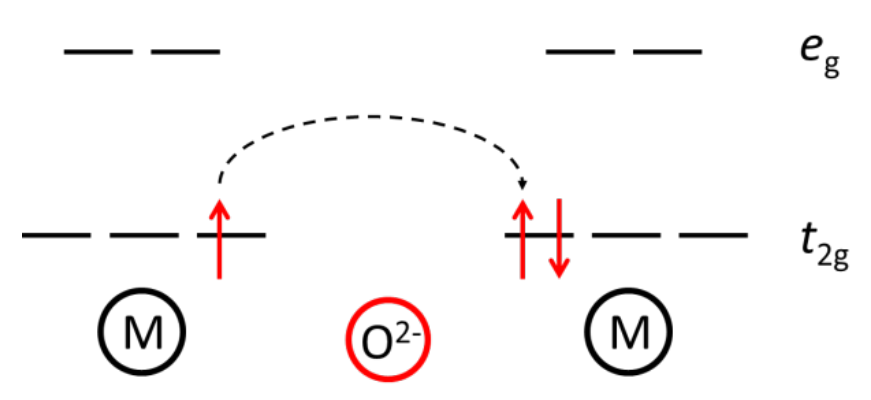

Figure 2-2: Schematic representation of the superexchange interaction as described by Anderson [26]. The degeneracy of the five $d$-orbitals of each metal ion (represented as black circle) is split according to an octahedral coordination as arbitrary example. Since the metal has $d^{1}$-configuration, there is one unpaired electron per metal ion in the unperturbed ground state. This state is mixed with excited states where one electron is "hopping" into an occupied neighboring orbital as represented by the dashed black arrow. 
simultaneously occur and Anderson also formulated two further but minor interactions which also have the form of equation (2-2) [26]. In the end, the different $J$ 's of all four interactions add up and if the sum is positive, the material is an antiferromagnet.

The simplest type of ferromagnetism or antiferromagnetism, respectively, occurs when only one nearest neighbor superexchange integral exists, as represented in panel (a) and (b) of Figure 2-3. For the sake of simplicity, this figure uses only 1D-spin-chains as example. If $J<0$, all spins are parallel aligned (a) to lower the free energy as much as possible according to equation (2-2). The same is true for $J>0$ where all neighboring spins are antiparallel aligned (b). Unfortunately, many materials have more than one relevant superexchange integral, resulting in a complex magnetic order. In fact, it is this complexity that is responsible for many properties of type-II multiferroics. The next step to enhance the simple model is to introduce a second superexchange integral $J$ ' which acts between next-nearest neighbors and has an opposite sign compared to $J$. It is not possible anymore to find a spin arrangement that gains the maximum possible energy for both interactions. Materials that exhibit this effect are called frustrated magnets. The resulting magnetic order depends on the relative magnitudes of $J$ and $J$ '. One possible example is an "up-up-down-down"-pattern that is shown in panel (c) of Figure 2-3. Each spin has one parallel and one antiparallel nearest neighbor, resulting in a vanishing contribution of $J$ to the free energy. The only energy gain is due to the nextnearest neighbor interactions where all spins are perfectly antiparallel aligned. If $J$ is stronger, a spin cycloid might have the lowest free energy as displayed in panel (d) of Figure 2-3. This kind of magnetic order is responsible for ferroelectricity in many type-II multiferroics as described in detail in chapter 2.2.1. In reality, crystals are three-dimensional and usually have more than two distinct superexchange interactions. The magnitude of the interactions and the resulting magnetic order strongly depends on the involved metals and the crystal structure. All materials that are investigated in this thesis are frustrated magnets. Details about their magnetic properties can be found in the according sections of chapter 2.3 .

(a)

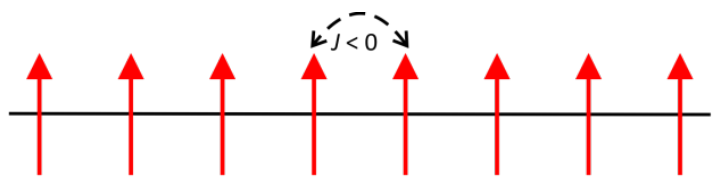

(c)

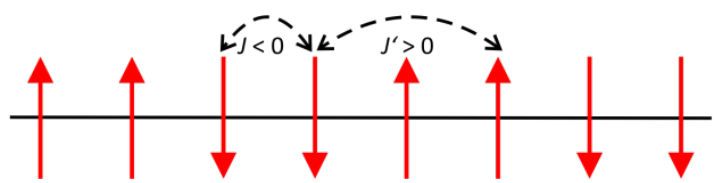

(b)

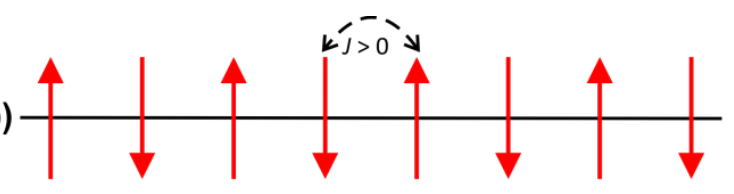

(d)

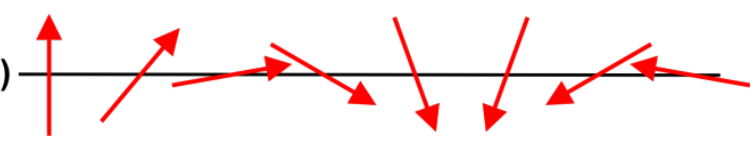

Figure 2-3: Schematic 1D-representation for different types of magnetic order. Red arrows are spins on different magnetic ions. The upper two panels display the simplest cases of ferromagnetism (a) and antiferromagnetism (b), respectively, with only one exchange/superexchange-integral $J$. If there are two competing J's with opposite sign, the resulting magnetic structure is frustrated and tends to be more complex. An "up-up-down-down"-pattern is presented in panel (c) while panel (d) shows a spin cycloid which is characteristic for many type-II multiferroics. This figure is inspired by [27]. 
The degree of magnetic frustration can be determined by the Curie-Weiß-law, which describes the magnetic susceptibility in the paramagnetic phase:

$$
\chi_{\mathrm{m}}=\frac{C_{\mathrm{CW}}}{T-T_{\mathrm{CW}}}
$$

$C_{\mathrm{CW}}$ is the Curie-Weiß-parameter while $T_{\mathrm{CW}}$ is the Curie-Weiß-temperature that indicates at which temperature magnetic interactions become relevant. This parameter has a negative sign and its absolute value is significantly higher than the actual ordering temperature $T_{\mathrm{N}}$ in the case of frustrated magnets. A so called frustration parameter is therefore introduced, which is the ratio of the absolute value of the Curie-Weiß-temperature and the Néel-temperature :

$$
\eta=\frac{\left|T_{\mathrm{CW}}\right|}{T_{\mathrm{N}}}
$$

\subsubsection{Ferroelectricity}

The textbook example for ferroelectrics is $\mathrm{BaTiO}_{3}$ which crystallizes at high temperatures in the cubic perovskite structure whose spatial center of inversion forbids a spontaneous polarization. There are several structural phase transitions at lower temperatures that break inversion symmetry and therefore allow electric polarization to occur [8]. This happens on a microscopic level by the displacement of ions with opposite charge in different directions, creating parallel aligned electric dipoles in each unit cell. This mechanism is of purely structural origin and cannot explain ferroelectricity in most multiferroics. Detailed explanations for several causes of ferroelectricity in multiferroics can be found in chapter 2.2.

\subsubsection{Ferrotoroidicty}

The existence of the fourth irreducible representation suggests that there is also a fourth primary ferroic order which is nowadays called ferrotoroidicity $[22,23]$. This concept is not much older than a decade and only a recent experiment might have actually confirmed it [28]. The order parameter is called toroidization $\boldsymbol{T}$ and is invariant under simultaneous space- and time-inversion but not under each inversion individually. In analogy to the magnetization $\boldsymbol{M}$ which can be induced by a circular current, the toroidization $\boldsymbol{T}$ is induced by a circular magnetization which in turn is induced by a toroidal current [22]. The role of ferrotoroidicity and its importance in the context of multiferroics is still under debate. Spaldin pointed out that many phenomena of type-II multiferroics, like the tilting of electric polarization under a magnetic field, belong to the same irreducible representation 
as ferrotoroidicity. Its introduction could also solve the exclusion problem that ferroelectricity and ferromagnetism have different inversion symmetries [27]. Schmid meanwhile admires ferrotoroidicity as "aesthetically satisfying" in terms of symmetry because it completes the group of examples for all four irreducible representations. On the other hand, he points out that the formulation of ferrotoroidicity might be rather unnecessary because all its effects could be explained as higher order expansions of the other three ferroics [23]. Due to this controversy and because it is not important for the experiments in this thesis, the concept of ferrotoroidicity will not be further pursued.

\subsubsection{Higher order ferroics}

The contribution to the free energy, as described by equation (2-1), can be viewed as the first term in a series expansion of the external field. Taking the actual four fields of Table 2-1 and expanding them to higher orders yields a number of cross-coupling expressions, some of which might be quite familiar [23]:

$$
\begin{aligned}
-F & =P_{(s) i} E_{i}+M_{(s) i} H_{i}+T_{(s) i} G_{i}+\varepsilon_{(s) i j} \sigma_{i j}+ \\
& +\frac{1}{2} \chi_{(e) i j} E_{i} E_{j}+\frac{1}{2} \chi_{(m) i j} H_{i} H_{j}+\frac{1}{2} \tau_{i j} G_{i} G_{j}+s_{i j k l} \sigma_{i j} \sigma_{k l}+ \\
& +\alpha_{i j} E_{i} H_{j}+\theta_{i j} E_{i} G_{j}+\zeta_{i j} H_{i} G_{j}+d_{i j k} E_{i} \sigma_{j k}+q_{i j k} H_{i} \sigma_{j k}+g_{i j k} G_{i} \sigma_{j k} \\
& +\beta_{i j k l} \sigma_{i j} H_{k} H_{l}+\beta_{i j k} E_{i} H_{j} H_{k}+\gamma_{i j k} H_{i} E_{j} E_{k} \cdots
\end{aligned}
$$

This equation describes field induced properties of the low-symmetry and low-temperature ferroic phase. The four expressions of the first line are the aforementioned primary ferroics. The index (s) denotes all spontaneously occurring order parameters. The second line contains all second order ferroics with uniform external field, namely electric permittivity, magnetic susceptibility, toroidic susceptibility and elastic compliance. All second-order cross-coupling terms are listed in line three. They are called linear magnetoelectric effect, electrotoroidic effect, magnetotoroidic effect, piezoelectric effect, piezomagnetic effect and piezotoroidic effect. Since the number of third-order ferroics is too long to be reasonably listed here, only three prominent examples are shown in line four. The first is magnetostriction, secondly the magnetobielectric effect and lastly the electrobimagnetic effect. All higher-order contributions containing the toroidic field are pure speculation till today. There is no experimental evidence for their existence and no microscopic theory. These terms only exist for symmetry reasons [23] and neglecting them simplifies equation (2-5): 


$$
\begin{aligned}
-F & =P_{(s) i} E_{i}+M_{(s) i} H_{i}+T_{(s) i} G_{i}+\varepsilon_{(s) i j} \sigma_{i j}+ \\
& +\frac{1}{2} \chi_{(e) i j} E_{i} E_{j}+\frac{1}{2} \chi_{(m) i j} H_{i} H_{j}+s_{i j k l} \sigma_{i j} \sigma_{k l}+\alpha_{i j} E_{i} H_{j}+d_{i j k} E_{i} \sigma_{j k}+q_{i j k} H_{i} \sigma_{j k}+ \\
& +\beta_{i j k l} \sigma_{i j} H_{k} H_{l}+\beta_{i j k} E_{i} H_{j} H_{k}+\gamma_{i j k} H_{i} E_{j} E_{k} \cdots
\end{aligned}
$$

In order to gain an expression for one of the four ferroic order parameters, the free energy has to be differentiated by the according external field. The electric polarization and magnetization, for example, can be derived as [3]:

$$
\begin{aligned}
& P_{i}=-\frac{\partial F}{\partial E_{i}}=P_{(s) i}+\frac{1}{2} \chi_{(e) i j} E_{j}+d_{i j k} \sigma_{j k}+\alpha_{i j} H_{j}+\beta_{i j k} H_{j} H_{k}+\gamma_{i j k} H_{j} E_{k} \cdots \\
& P_{(s) i}, E_{j}, \sigma_{j k}=0 \\
& =\alpha_{i j} H_{j}+\beta_{i j k} H_{j} H_{k} \\
& M_{i}=-\frac{\partial F}{\partial H_{i}}=M_{(s) i}+\frac{1}{2} \chi_{(m) i j} H_{j}+q_{i j k} \sigma_{j k}+\beta_{i j k l} \sigma_{j k} H_{l}+\alpha_{i j} E_{j}+\beta_{i j k} E_{j} H_{k}+\gamma_{i j k} E_{j} E_{k} . \\
& M_{(s) i}, H_{j}, \sigma_{j k}=0 \\
& =\alpha_{i j} E_{j}+\gamma_{i j k} E_{j} E_{k}
\end{aligned}
$$

The second line of equation (2-7) shows the contributions to the electric polarization when the only external field is a magnetic one and the material is not ferroelectric. A similar expression can be derived for the magnetization when only an electric field is applied, as shown in the second line of equation (2-8). In both cases, the remaining terms are the linear and second-order magnetoelectric effect, respectively [3]. This example demonstrates how expressions for the different interactions can be derived from the series expansion of equation (2-6).

It is important to point out that some names for the terms in equation (2-5) are used rather ambiguously. Two examples are "magnetoelectric effect" and "magnetostriction". The expression linear magnetoelectric effect was originally used for the induction of an electric polarization or magnetization by the respective opponent field according to equation (2-7) and (2-8). The discovery of Kimura that it is possible to tilt the electric polarization when a magnetic field is applied [11] was also called magnetoelectric effect, but is something fundamentally different on a microscopic level. In this case, the magnetic field induces a phase transition which alters the magnetic structure and therefore leads to a different orientation of the electric polarization. The only similarity in both cases is that the magnetic field changes the electric properties. This confusion is also surrounding the name "magnetostriction" which originally meant the deformation of a crystal when a magnetic field is applied (see equation (2-5)). The same expression, however, is used when the deformation happens during a magnetic phase transition. The onset of the magnetic ordering can change the crystal structure because the magnitude of the superexchange interaction between two spins depends on 
the relative orientation of the respective ions. More about this aspect of magnetostriction can be found in chapter 2.2.2. 


\subsection{Multiferroicity}

Single phase multiferroics can be divided into two categories that are distinguished by the mechanism that causes ferroelectricity $[9,10,27,29]$. Type-I multiferroics formally exhibit magnetic as well as electric order in one phase, but the origins for both are quite different and therefore there are two distinct phase transitions at remote temperatures. The ferroelectric transition usually takes place at temperatures hundreds of Kelvin higher than the magnetic transition and, even more important, the electric properties do not significantly change with the onset of magnetic ordering. Unsurprisingly, the coupling between electric and magnetic properties is not stronger than in any ordinary material. In Type-II multiferroics, on the other hand, ferroelectricity emerges during a magnetic phase transition what indicates a close connection between both ordering phenomena.

Both categories of multiferroics are no homogeneous groups, but can be further subdivided. Since type-I multiferroics are not the focus of this thesis, only three examples shall be mentioned at this point (which are summarized in more detail in [10]). First, there are "lone pair" multiferroics containing $\mathrm{Bi}$ - or $\mathrm{Pb}$-atoms whose closed $6 s$-shell is highly polarizable and can therefore create local dipoles that order to a macroscopic polarization. Prominent examples are $\mathrm{BiFeO}_{3}$ and $\mathrm{BiMnO}_{3}$ that both show huge differences between their respective ferroelectric $\left(T_{\mathrm{FE}}=1100\right.$ and $\left.760 \mathrm{~K}\right)$ and antiferromagnetic phase transition temperatures $\left(T_{\text {AFM }}=643\right.$ and $\left.105 \mathrm{~K}\right)[9]$.

The second group is called charge-ordered multiferroics, what can be interpreted in two ways. On the one hand, there are ordered charges on the atoms because these materials, like $\mathrm{Pr}_{0.5} \mathrm{Ca}_{0.5} \mathrm{MnO}_{3}$ and $\mathrm{LuFe}_{2} \mathrm{O}_{4}$, simply contain differently charged ions. Furthermore, the electron density of chemical bonds between ions is ordered in such a way that successive bonds are differently charged. This leads to an alternating contraction and elongation of bonds, resulting in dimers which act as local dipoles because of the differently charged ions. Collective ordering of these dipoles can break spatial inversion symmetry and lead to ferroelectricity. This mechanism is similar to that of a group of type-II multiferroics which will be discussed in more detail in chapter 2.2.2.

Lastly, there are hexagonal manganites like $\mathrm{YMnO}_{3}$ which exhibit a structural phase transition where a rotation of $\mathrm{MnO}_{6}$-octahedra breaks spatial inversion symmetry and thereby induces spontaneous polarization. This mechanism is similar to classic ferroelectricity like in $\mathrm{BaTiO}_{3}$.

The ferroelectricity is in all three cases of electronic and structural origin while magnetic ordering rather coincidently appears. This is in contrast to type-II multiferroics where magnetism is the reason for ferroelectricity. According to the current state of research, there are two mechanisms that can describe this coupling: Anisotropic exchange interaction in cycloidal spin arrangements and 
charge-ordering due to magnetostriction of collinear antiferromagnets. Both phenomena are explained in the following two sub-chapters.

\subsubsection{Dzyaloshinski-Moriya interaction}

Equation (2-2) is a valid description of both, exchange and superexchange interaction, but it is only a first approximation, depending on the point group. There are many phenomena that can only be explained with a relativistic correction as introduced by Moriya $[30,31]$ :

$$
F_{\mathrm{ex}}=\sum_{\mathrm{i}, \mathrm{j}} \mathrm{J}_{\mathrm{ij}}\left(\boldsymbol{S}_{\mathrm{i}} \cdot \boldsymbol{S}_{\mathrm{j}}\right)+\boldsymbol{D} \cdot\left(\boldsymbol{S}_{\mathrm{i}} \times \boldsymbol{S}_{\mathrm{j}}\right)
$$

In order to distinguish both interactions, they are usually referred to as isotropic superexchange interaction $\left(F_{\mathrm{ex}, \mathrm{sso}}\right)$ and anisotropic superexchange interaction $\left(F_{\mathrm{ex}, \text { aniso }}\right)$. The relativistic correction can also be applied to the exchange interaction which leads to the same expression as in equation (2-9). This correction is a result of spin-orbit-coupling and therefore much smaller than the isotropic term.

At this point it might be important to make some notes about the names of these interactions. Since the exchange integral $J$ is a scalar quantity, it is possible to equally rotate all spins in the same direction with respect to the crystal lattice without changing the energy contribution $F_{\mathrm{ex}, \text { iso }}$ because the relative orientation of the spins remains constant and therefore also the product $S_{i} \cdot S_{j}$. Hence, this interaction has the name isotropic exchange. Anisotropic exchange, on the other hand, also depends on the orientation of the spins towards the vector $\boldsymbol{D}$ which is in turn dictated by crystal symmetry. A uniform rotation of all spins will therefore alter $F_{\mathrm{ex}, \text { aniso. }}$. This is also true for a third interaction called "single-ion anisotropy" (SIA) that should not be confused with anisotropic exchange. SIA is a product of the interaction of orbitals with the crystal field since the coordination geometry will lift the degeneracy of, for example, $d$-orbitals. Those orbitals that are occupied by unpaired electrons will determine the preferred orientation of spins, called "easy-axis". SIA contributes to the free energy by a term like [32]:

$$
F_{\mathrm{SIA}}=\sum_{\mathrm{i}} b_{\mathrm{i}} S_{\mathrm{i}, \text { easy }}^{2}
$$

where $S_{i, \text { easy }}$ is the component of the $\mathrm{i}^{\text {th }}$ spin along the easy-axis.

Anisotropic superexchange was formulated by Moriya to build a quantum mechanical foundation for a phenomenological approach by Dzyaloshinsky to explain the occurrence of weak ferromagnetism in the trigonal antiferromagnet $\alpha-\mathrm{Fe}_{2} \mathrm{O}_{3}$ [33]. Isotropic superexchange dominates the magnetic structure and favors a perfect antiparallel alignment of spins. The anisotropic contribution, 


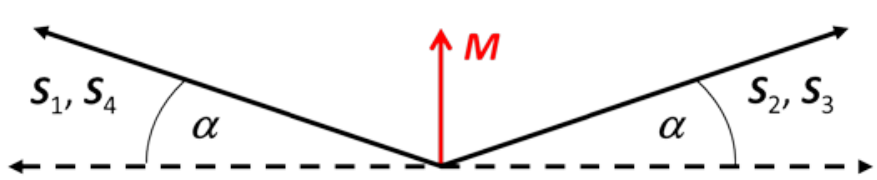

Figure 2-4: Schematic representation of the spin tilt in antiferromagnetic $\alpha-\mathrm{Fe}_{2} \mathrm{O}_{3}$, viewing along the trigonal [111]-axis. All four spins $\left(\boldsymbol{S}_{\mathrm{i}}\right)$ of the unit cell are antiparallel aligned in an up-down-down-up manner if only isotropic superexchange exists (dashed black arrows). Anisotropic superexchange slightly tilts the spins (solid black arrows) and creates a macroscopic magnetization (solid red arrows). The tilt angle $\alpha$ is exaggerated for demonstration purposes. This figure is inspired by [33].

on the other hand, vanishes as long as the spins are collinear and can therefore only lower the total free energy if all spins are slightly tilted in the same direction (see Figure 2-4). The isotropic term is proportional to the cosine of the tilt angle $\alpha$ and remains almost constant if $\alpha$ is small. The minimum of the total free energy is therefore reached at small but finite values of $\alpha$. This uniform tilt gives rise to a small macroscopic magnetization $\boldsymbol{M}_{(\mathrm{s})}$. Dzyaloshinski showed that the tilt is permitted by symmetry and he could also estimate the magnitude of $\boldsymbol{M}_{(\mathrm{s})}$ in the framework of Landau's theory.

Anisotropic superexchange is used as an explanation for the occurrence of ferroelectricity in non-collinear antiferromagnets and named Dzyaloshinsky-Moriya interaction (DMI). In fact, there are three theories that make slightly different assumptions but all come to the same conclusion:

$$
\boldsymbol{P} \propto \boldsymbol{e}_{\mathrm{ij}} \times\left(\boldsymbol{S}_{\mathrm{i}} \times \boldsymbol{S}_{\mathrm{j}}\right)
$$

Katsura developed the "spin current model" [13] where he assumed that two magnetic ions are bonded via an oxygen atom (see Figure 2-5) and carry the spins $\boldsymbol{S}_{\mathrm{i}}$ and $\boldsymbol{S}_{\mathrm{j}}$. Due to the angle between both spins, a spin current $j_{\mathrm{s}}$ is induced, which is proportional to $S_{\mathrm{i}} \times S_{\mathrm{j}}$ and flows from one magnetic ion to the next along the connecting vector $\boldsymbol{e}_{\mathrm{ij}}$. In analogy to moving charges that create a magnetic field, this spin current induces an electric polarization [34]. This theory is quite elegant in a way as it does not rely on any structural changes in form of atomic displacements since the polarization is of pure electronic origin.

This is in contrast to the second theory, proposed by Sergienko [14], who explains the polarization by a displacement of negatively charged oxygen ions due to magnetostriction. He used the paramagnetic crystal structure of $\mathrm{TbMnO}_{3}$ and constructed a Hamiltonian that describes the superexchange interaction of the cycloidal spin ordering. The next step was minimizing the total free energy by shifting oxygen atoms. The isotropic superexchange favors an alternating displacement

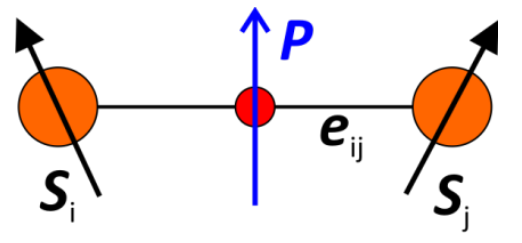

Figure 2-5: Schematic representation of the electric polarization $\boldsymbol{P}$ (blue vector) induced by Dzyaloshinsky-Moriya interaction. Orange spheres are magnetic metal ions that carry the spins $\boldsymbol{S}_{\mathrm{i}}$ and $\boldsymbol{S}_{\mathrm{j}}$ (black vectors). The red sphere is an oxygen ion located on the connection vector $\boldsymbol{e}_{\mathrm{ij}}$. This figure is inspired by [13]. 
pattern of successive atoms that does not change the balance point of negative charges (see Figure 2-6, upper panel). This is different when considering the anisotropic contribution that prefers a shift of all oxygens in the same direction which breaks inversion symmetry and gives rise to macroscopic polarization (see Figure 2-6, lower panel).

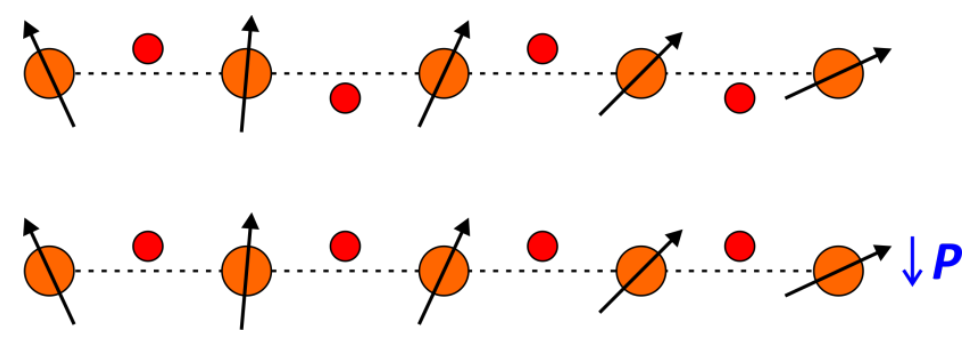

Figure 2-6: Schematic representation of the oxygen displacement pattern induced by isotropic (upper panel) and anisotropic superexchange (lower panel). Orange spheres are magnetic metal ions with black arrows as spins. Red spheres represent oxygen ions.

While the first two models rely on quantum mechanics to explicitly incorporate the anisotropic superexchange, Mostovoy used Landau's theory like Dzyaloshinsky [12]. This model yields the same expression like equation (2-11), but it technically does not include DMI as an interaction between spins. It only relies on isotropic superexchange to explain the spin cycloid due to magnetic frustration as previously mentioned in chapter 2.1.1.1. In a second step, the cycloid breaks spatial inversion symmetry and an energy term arises that induces polarization and has the same form like DMI. This is in contrast to the models of Katsura and Sergienko, who both explicitly mention that the anisotropic exchange plays a vital role in stabilizing the spin cycloid, like in $\alpha-\mathrm{Fe}_{2} \mathrm{O}_{3}$ where DMI stabilizes the misalignment of spins.

Although Mostovoy's theory might not be as accurate on a microscopic level, it makes a prediction that is true for many multiferroics materials. Since electric polarization is only induced by a non-collinear spin arrangement, at least two basis vectors are needed to describe the magnetic ordering. They are called primary and secondary easy-axis and span the cycloidal plane. Both axes are separate order parameters in Landau's theory and so there are also two successive phase transitions. Consequently, there is the first transition at $T_{\mathrm{N}}$ with a collinear antiferromagetic ordering along the primary easy-axis. An additional magnetic component along the secondary easy-axis emerges at the second transition giving rise to the spin cycloid. If there is no strong magnetic anisotropy, which would manifest in an elliptic cycloid, these temperatures are close together.

If the previous considerations about the direction of the polarization shall be applied to a real sample with three-dimensional crystal structure, equation (2-11) leaves an important question unanswered: what is the direction of $\boldsymbol{e}_{\mathrm{ij}}$ ? It is quite obvious when all atoms are schematically drawn on a simple line, but in reality there are neighboring atoms in different directions. $\boldsymbol{e}_{\mathrm{ij}}$ rather 
corresponds to the propagation wave vector of the spin spiral $\boldsymbol{k}[10,27]$. Equation (2-11) can therefore be rewritten as:

$$
\boldsymbol{P} \propto \boldsymbol{k} \times\left(\boldsymbol{S}_{\mathrm{i}} \times \boldsymbol{S}_{\mathrm{j}}\right)=\boldsymbol{k} \times \boldsymbol{C}
$$

Here, $\boldsymbol{C}$ is the normal vector of the cycloidal plane and therefore perpendicular to both easyaxes of the magnetic structure. There are two extreme cases for the relative orientation of $\boldsymbol{k}$ and $\boldsymbol{C}$. In the case of a proper cycloid, as represented in Figure 2-7-a, $\boldsymbol{k}$ lies in the cycloidal plane and is therefore perpendicular to $\boldsymbol{C}$. If $\boldsymbol{k}$ is, on the other hand, perpendicular to the rotational plane and therefore parallel to $\boldsymbol{C}$ as depicted in Figure 2-7-b, it is usually referred to as "proper helix" or "proper screw". Obviously, spontaneous polarization vanishes in this case. Generally, arbitrary angles between $\boldsymbol{C}$ and $\boldsymbol{k}$ are possible.

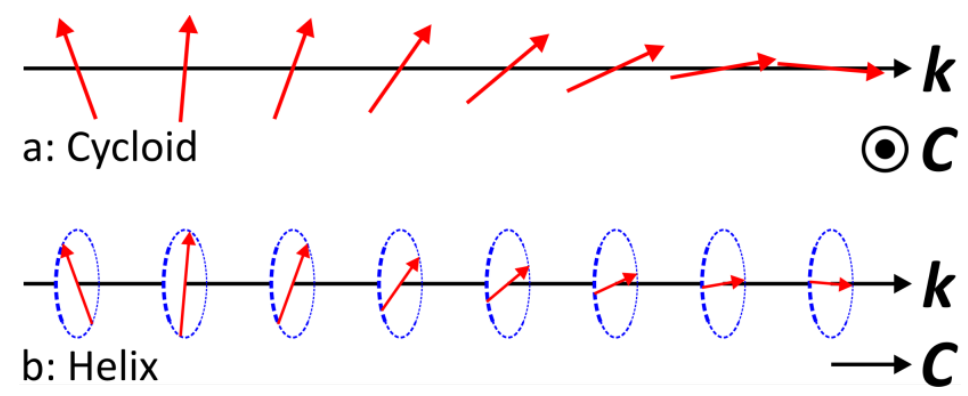

Figure 2-7: Schematic representation of a spin cycloid (upper panel) and a spin helix (lower panel). Red arrows are spins and long black arrows are the propagation vectors of the spiral $\boldsymbol{k}$. The normal vector of the spiral rotation plane $\boldsymbol{C}$ is perpendicular to $\boldsymbol{k}$ in the case of the cycloid and parallel to $\boldsymbol{k}$ in the case of the helix, respectively. This figure is inspired by [35].

The sign of $\boldsymbol{C}$ is an indicator for the chirality of the spin cycloid and directly determines the direction of $\boldsymbol{P}$. This means that the chirality has to change when $\boldsymbol{P}$ is reversed by an applied electric field. Similarly, $\boldsymbol{P}$ is reversed when the chirality is switched with a magnetic field. This underlines the tight connection of magnetic and electric properties in these type-II multiferroics.

\subsubsection{Charge-ordering by magnetostriction}

Dzyaloshinsky-Moriya interaction cannot be the driving force for ferroelectricity of type-II multiferroics with collinear antiferromagnetic ordering. In this case, another mechanism comes into play which bases on magnetostriction and is similar to charge-ordering of type-I multiferroics $[27,29]$. These materials need two or more differently charged magnetic ions as necessary requirement, either different elements or one element with different oxidation states. The easiest way to explain charge-ordering is the one-dimensional chain as shown in Figure 2-8. The upper panel is the undistorted chain without magnetostriction where all ions have the same bond distance and no 
macroscopic polarization can occur due to a centers of inversion located on each atom. In this exemplary case, the nearest-neighbor interactions are ferromagnetic while the next-nearestneighbor interaction favors antiparallel spins. This leads to a frustrated compromise structure with characteristic up-up-down-down pattern (see also chapter 2.1.1.1). The contribution of the magnetic order to the total free energy $F$ can then be expressed by [29]:

$$
F=\sum_{i} J_{F M}\left(r_{i, i+1}\right)\left(S_{i} \cdot S_{i+1}\right)+J_{A F M}\left(r_{i, i+2}\right)\left(S_{i} \cdot S_{i+2}\right)
$$

Please note, that $J_{\mathrm{FM}}<0$ and $J_{\mathrm{AFM}}>0$ are in general a function of the distance $\boldsymbol{r}_{\mathrm{i}, \mathrm{j}}$ between the according pair of ions. Since each spin has one parallel and one antiparallel nearest neighbor spin, all contributions of $J_{\mathrm{FM}}$ cancel out. This magnetic degeneracy can be lifted by displacing the ion towards one of its neighbors. If the distance to the ion with parallel spin is decreased, the absolute value of the according $J_{F M}$ will increase and therefore $F$ is lowered. The bond to the ion with antiparallel spin is consequently elongated, resulting in an absolute decrease of $J_{\mathrm{FM}}$ and a further lowering of $F$. The magnetic ordering therefore favors a dimerization of ions with parallel spins as it is presented in the lower panel of Figure 2-8. Each dimer is also an electric dipole since neighboring ions carry different charges. The dimerization breaks inversion symmetry and therefore creates a macroscopic polarization. The upper panel can be regarded as the paramagnetic crystal structure while the dimers in the lower panel form during the antiferromagnetic phase transition. This displacement of atoms induced by magnetic ordering is called magnetostriction and highlights the type-II nature of this multiferroic chain.

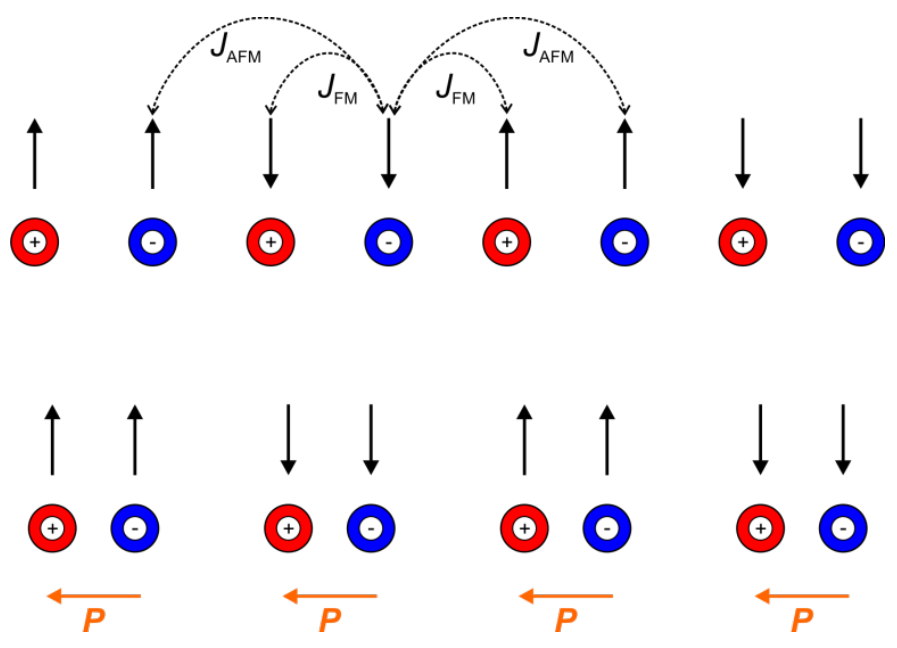

Figure 2-8: Schematic representation of charge-ordering by magnetostriction applied to a linear chain with ferromagnetic nearest-neighbor $\left(J_{\mathrm{FM}}<0\right)$ and antiferromagnetic next-nearest-neighbor superexchange interactions $\left(J_{\text {AFM }}>0\right)$. Red and blue spheres are magnetic ions with different charges and black arrows represent the according spins. Ions with parallel spins decrease their distance and form dimers with local dipole moment which add up to a macroscopic polarization $\boldsymbol{P}$. This figure is inspired by [29]. 
This 1D model is of course a crude oversimplification with the sole purpose of demonstrating the concept. In the real 3D case, there are J's between ion pairs in all three directions and they do not only depend on distances, but also bond angles between magnetic ions and diamagnetic atoms like oxygen [36]. Possible displacements induced by magnetostriction can therefore also include the distortions or rotation of whole building blocks of the crystal structure. Hence, there is no simple blueprint explanation that can be applied to every material because the crystal and magnetic structure is different in each case. Ferroelectricity in $\mathrm{R}_{2} \mathrm{Mn}_{4} \mathrm{O}_{10}$-compounds is commonly ascribed to originate from this mechanism and will be further explained in chapter 2.3.3.3. 


\subsection{Materials}

Three different materials were investigated in this thesis: manganese tungstate $\left(\mathrm{MnWO}_{4}\right)$, cupric oxide $(\mathrm{CuO})$ and bismuth manganate $\left(\mathrm{Bi}_{2} \mathrm{Mn}_{4} \mathrm{O}_{10}\right)$. The next subchapters will provide an overview over important properties and will discover similarities and differences between the substances.

\subsection{1 $\mathrm{MnWO}_{4}$}

$\mathrm{MnWO}_{4}$ is also known under its mineral name Hübnerite. It serves as a model substance for the investigation of multiferroicity induced by spiral spin order. All relevant properties like crystal structure, magnetic structure, phase transitions and the origin of ferroelectricity will be discussed in the following subsections.

\subsubsection{Crystal structure}

The crystal structure of $\mathrm{MnWO}_{4}$ was described in detail 50 years ago [37]. It crystallizes in the monoclinic space group $P 2 / c$ with lattice parameters $a=4.8226(3) \AA, b=5.7533(6) \AA, c=4.9923(5) \AA$ and $\beta=91.075(7)^{\circ}$ and with two chemical formula units per unit cell [38]. The relative coordinates of all atoms of the asymmetric unit cell can be found in Table 2-2. The oxygen ions form a distorted hexagonal close packed structure with some octahedral sites filled by manganese and tungsten ions. $\mathrm{Mn}^{2+}$ has $3 d^{5}$ high-spin electron configuration with a total spin of $S=2.5$ and $\mathrm{W}^{6+}$ has noble gas configuration without a magnetic moment. The coordination octahedra of both metals share common edges with neighboring octahedra of the same type of metal ion and form infinite zig-zag chains along the $c$-axis (see Figure 2-9, left and right) which are connected to chains of the other type of metal ion via single oxygen atoms at corners (see Figure 2-9, bottom). All metal atoms are part of layers with normal vector along the $\boldsymbol{a}$-axis which alternatingly consist of manganese and tungsten, respectively (see Figure 2-9, right and bottom). Furthermore, there are glide mirror planes perpendicular to the $\boldsymbol{b}$-axis with translation in $\boldsymbol{c}$-direction (see Figure 2-9, left).

The crystal structure is reflected by the anisotropy of the isothermal compressibility: $\kappa_{\mathrm{b}}>\kappa_{\mathrm{a}}>\kappa_{\mathrm{c}}$ [39]. With increasing pressure, the octahedral coordination around $\mathrm{Mn}^{2+}$ becomes distorted and compressed while the distance between $\mathrm{W}^{6+}$ and its surrounding oxygen atoms is much less pressure dependent. The stiff $\mathrm{W}$-octahedra chains propagate along the $\boldsymbol{c}$-axis which turns out to be the most rigid direction. In $\boldsymbol{b}$-direction on the other hand, the chains are only loosely interconnected, making this the most easily compressible axis. The distance between $\mathrm{Mn}$ - and W-ions therefore decreases with increasing pressure. 
Table 2-2: Relative coordinates of all atoms of the asymmetric unit cell of $\mathrm{MnWO}_{4}$ [38].

\begin{tabular}{|c|c|c|c|}
\hline & $\boldsymbol{x}$ & $\boldsymbol{y}$ & $\boldsymbol{z}$ \\
\hline $\mathrm{Bi}$ & 0.5 & $0.6853(4)$ & 0.25 \\
\hline $\mathrm{Mn}^{4+}$ & 0 & $0.1795(4)$ & 0.25 \\
\hline $\mathrm{O}_{1}$ & $0.2108(3)$ & $0.1024(2)$ & $0.21(3)$ \\
\hline $\mathrm{O}_{2}$ & $0.2516(2)$ & $0.3752(2)$ & $0.25(2)$ \\
\hline
\end{tabular}
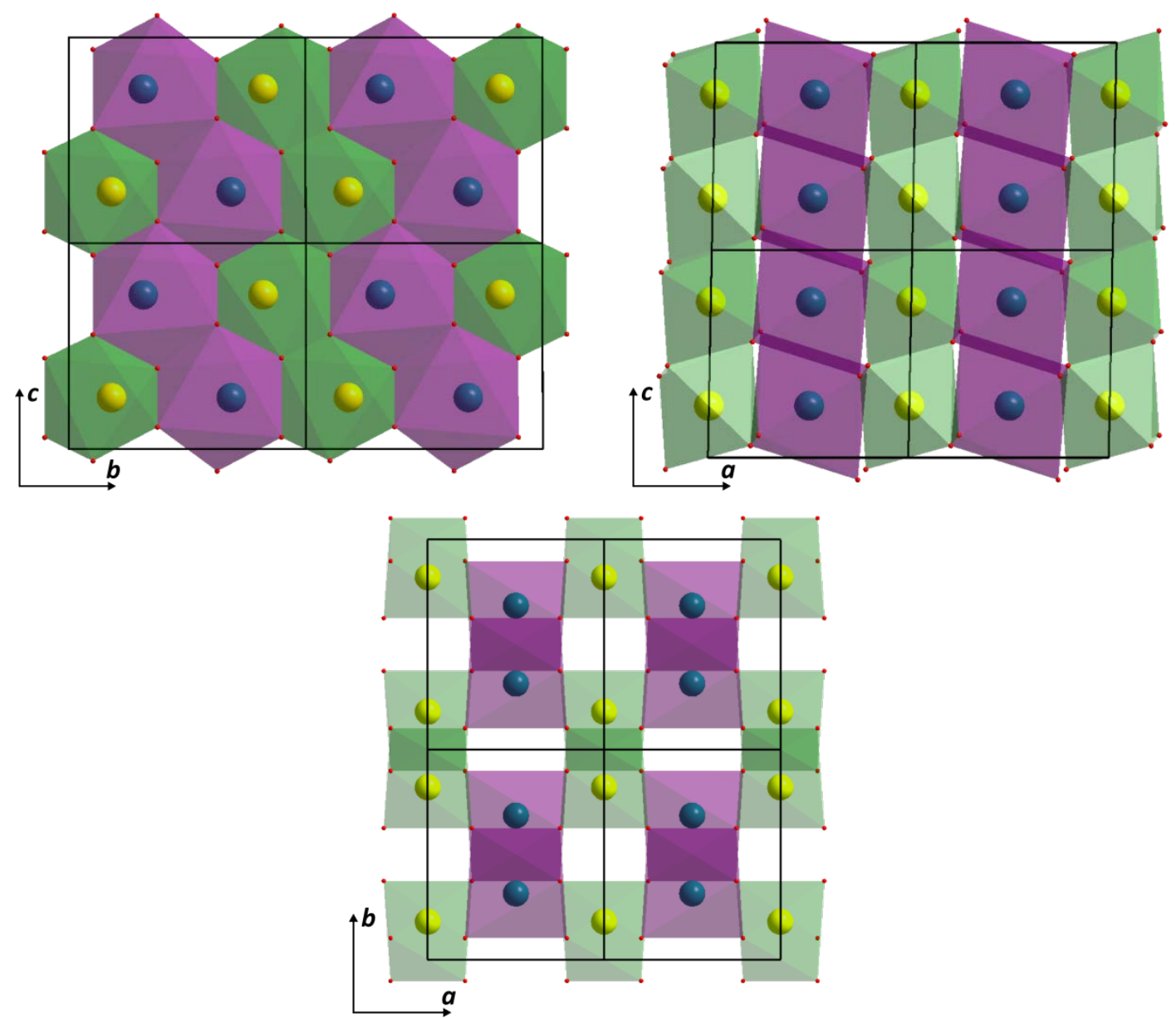

Figure 2-9: Crystal structure of $\mathrm{MnWO}_{4}$ with view along the $\boldsymbol{a}$-axis (left), $\boldsymbol{b}$-axis (right) and $\boldsymbol{c}$-axis (bottom), respectively. Yellow spheres are $\mathrm{W}^{6+}$-ions, octahedrally coordinated by oxygen atoms (small red spheres) as indicated by green color. Purple octahedra represent the coordination geometry around $\mathrm{Mn}^{2+}$-ions (green spheres). Crystallographic data are taken from [38].

\subsubsection{Magnetic structure}

$\mathrm{MnWO}_{4}$ exhibits three successive antiferromagnetic phase transitions at temperatures $T_{\mathrm{N} 1}=6.4-8.0 \mathrm{~K}, T_{\mathrm{N} 2}=12.3 \mathrm{~K}$ and $T_{\mathrm{N} 3}=13.5 \mathrm{~K}[35,38,40]$. The first two transitions on cooling at $T_{\mathrm{N} 3}$ and $T_{\mathrm{N} 2}$ are of second order nature while a first order transition takes place at $T_{\mathrm{N} 1}[35,38]$. There are several different values reported for $T_{\mathrm{N} 1}$ in a range of two Kelvin, depending on the quality of the sample. Impurities generally tend to shift this transition to lower temperatures [35]. 
There are two major axes for the magnetic moments to align. The primary easy-axis lies in the $\boldsymbol{a c}$-plane with an angle of $37^{\circ}$ towards the $\boldsymbol{a}$-axis while the secondary easy-axis coincides with the crystallographic $\boldsymbol{b}$-axis [38]. The direction perpendicular to both easy-axes is called "hard-axis". Since the ligand field splitting is weaker than the spin-pairing energy, $\mathrm{Mn}^{2+}$ occurs in high-spin configuration where each $d$-orbital is occupied by a single unpaired electron. The electron configuration therefore has, in first approximation, octahedral symmetry $O_{\mathrm{h}}$, leading to a weak single-ion anisotropy in the same order of magnitude like the anisotropic superexchange. Both interactions are weaker than the isotropic superexchange. The directions of the easy-axes are therefore primarily dictated by superexchange interactions [41].

The antiferromagnetic phase between $T_{\mathrm{N} 2}$ and $T_{\mathrm{N} 3}$ is called AF3 and exhibits an incommensurate magnetic modulation with $\boldsymbol{k}_{\mathrm{CC}}=(-0.2165,0.5,0.4585)[38,42]$. The spins are pointing along the primary easy-axis with a sinusoidal modulation of the amplitude (see Figure 2-10, right) [38]. Furthermore, a recent neutron diffraction study revealed that the spins also have a slight $\boldsymbol{b}$-component giving rise to a minor cycloid. Since there are two $\mathrm{Mn}$-ions in the crystallographic unit cell, the magnetic structure can be regarded as two spin sublattices. The chirality of both sublattices is of opposite sign and the overall magnetic structure therefore still obeys spatial inversion symmetry [43].

Between $T_{\mathrm{N} 1}$ and $T_{\mathrm{N} 2}$, there is the antiferromagnetic phase AF2 with the same temperatureindependent modulation vector $\boldsymbol{k}_{\mathrm{IC}}$ like AF3 $[38,42]$. An additional spin-component arises along the secondary easy-axis which reaches $90 \%$ of the magnitude along the primary easy-axis $[38,44]$. The components of both easy-axes have an anti-phase relation that creates a slightly elliptic cycloid

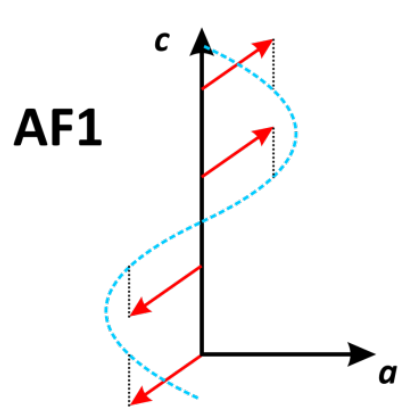

commensurate sinoidal

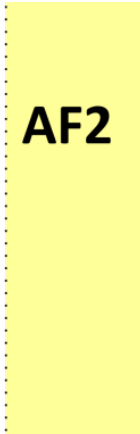

AF2

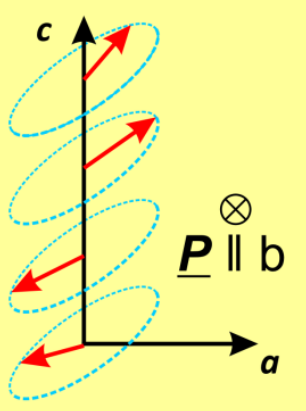

incommensurate

cycloidal

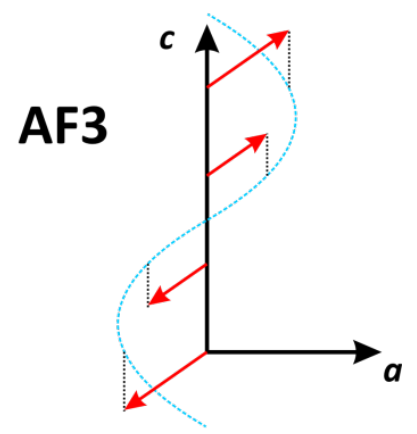

sinoidal

$$
T_{\mathrm{N} 1} \approx 8 \mathrm{~K}
$$

$$
T_{\mathrm{N} 3} \approx 13.5 \mathrm{~K}
$$

Figure 2-10: Schematic representation of the magnetic structure of all three antiferromagnetic phases. Red arrows are spins while the origin of the arrow is the position of the according $\mathrm{Mn}^{2+}$-ion. Spins of two crystallographic unit cells in $c$-direction are presented. Please note that the cycloidal plane in the middle panel is slightly tilted for better visualization. 
rotating in the plane perpendicular to the hard-axis (see Figure 2-10, middle). In contrast to AF3, the chirality of both $\mathrm{Mn}$-ions in the unit cell is the same and therefore inversion symmetry is broken [43], giving rise to spontaneous polarization as described in detail in chapter 2.3.1.3.

At the first order phase transition at $T_{\mathrm{N} 1}$, the modulation of the spins becomes commensurate with $\boldsymbol{k}_{\mathrm{C}}=(-0.25,0.5,0.5)[37,38,42]$. This low-temperature phase AF1 is the magnetic ground state of $\mathrm{MnWO}_{4}$ with a characteristic up-up-down-down pattern along the Mn-octahedra chains in c-direction (see Figure 2-10, left).

The commensurate modulation vector $\boldsymbol{k}_{\mathrm{c}}$ describes a $4 \times 2 \times 2$-fold magnetic supercell. The spin of an arbitrary $\mathrm{Mn}$-ion has the same phase and therefore same direction as the spin of a translational equivalent $\mathrm{Mn}$-ion in any other supercell. This is not true anymore in the incommensurate phases. The misfit vector $\Delta \boldsymbol{k}=\boldsymbol{k}_{\mathrm{C}}-\boldsymbol{k}_{\mathrm{C}}=(-0.034,0,0.042)$ induces a phase shift between former equivalent spins which results in a change of amplitude in the AF3-phase or a rotation in the cycloidal plane for AF2, respectively. This can be seen in the left panel of Figure 2-11 where the magnetic structure of the AF2-phase is depicted. The upper two unit cells would be identical to the lower two unit cells in the commensurate phase, but their respective spins are
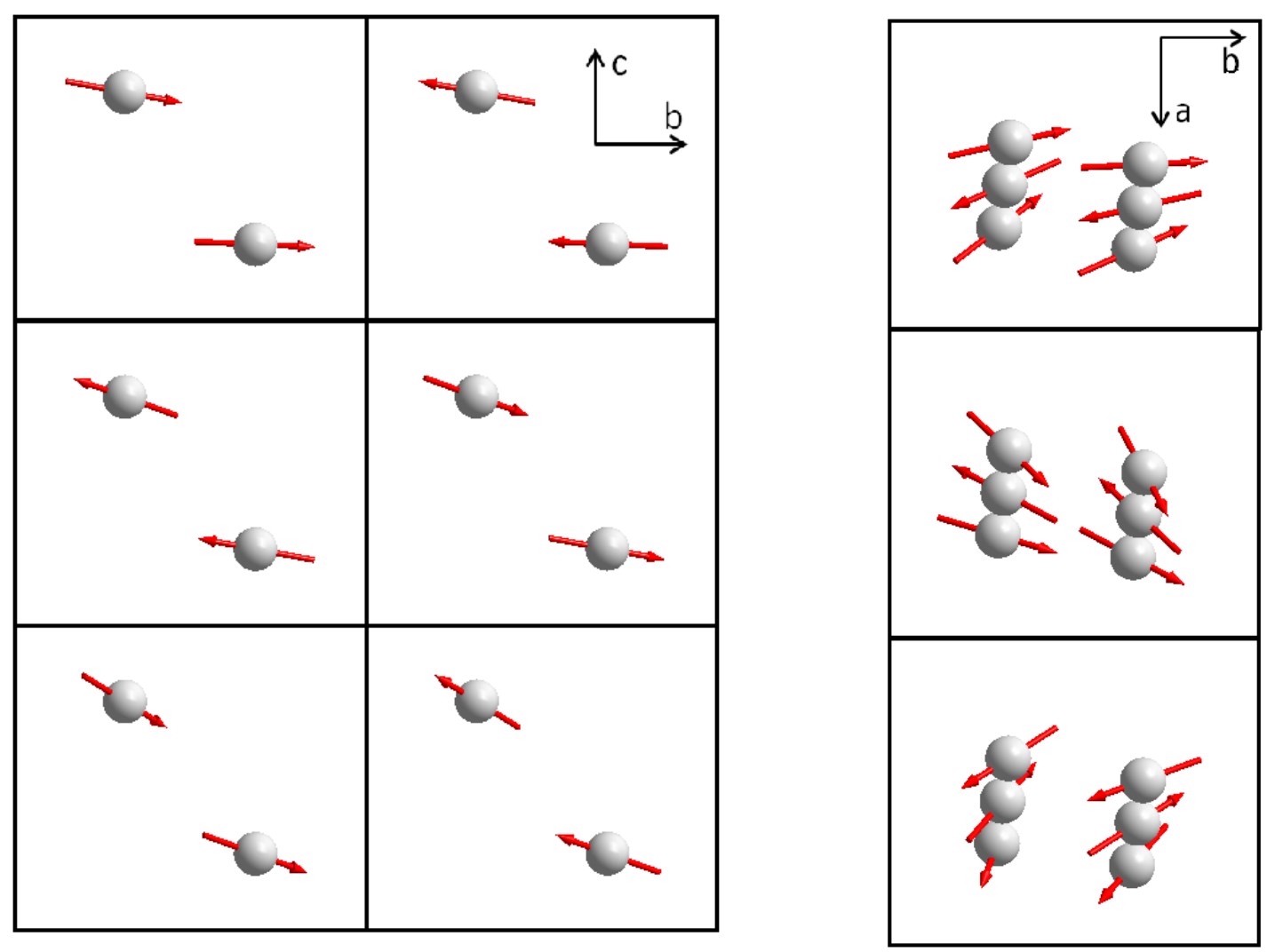

Figure 2-11: Magnetic structure of $\mathrm{MnWO}_{4}$ in the AF2-phase with view along the $\boldsymbol{a}$-axis (left) and approximately along the $\boldsymbol{c}$-axis (right), respectively. White spheres are $\mathrm{Mn}$-atoms and their according spins are represented as red arrows. Please note, that the viewing direction in the right panel is slightly tilted away from the $\boldsymbol{c}$ - to the $\boldsymbol{a}$-axis because rear $\mathrm{Mn}$-atoms would otherwise be hidden. Crystallographic data are taken from [38]. 
slightly rotated in the AF2-phase. Please note that the effect of the discommensuration is only a minor alteration of the commensurate structure (see also Figure 2-11, right). Spins in, for example, the upper unit cell are still roughly antiparallel and parallel to spins in the middle and lower unit cell, respectively. Spins of neighboring unit cells in $\boldsymbol{b}$-direction, however, are exactly antiparallel since the $\boldsymbol{b}$-component of $\boldsymbol{k}_{\mathrm{IC}}$ is still commensurate.

Several inelastic neutron scattering studies determined spectra of magnons with different propagation directions and fitted a theoretical model to the experimental data to determine the magnitude of superexchange $[32,45,46]$. The model consists of an energy term for the single-ion anisotropy and up to 12 isotropic superexchange integrals. It is not sufficient to restrain the model to interactions only along the Mn-chains in c-direction, but it is necessary to take even next-nextnearest neighbor interactions between neighboring chains into account, especially in $\boldsymbol{a}$-direction. All superexchange integrals favor an antiparallel alignment of spins. It can be concluded, that $\mathrm{MnWO}_{4}$ has a highly frustrated, three-dimensional spin system with long-ranging interactions. Frustration primarily appears along the $\boldsymbol{a}$ - and $\boldsymbol{c}$-axis which is evident by the incommensurate $\boldsymbol{a}$ - and $c$-component of $\boldsymbol{k}_{\mathrm{IC}}$ in the AF2 and AF3-phase [32]. The interactions between neighboring chains in $\boldsymbol{b}$-direction, on the other hand, are rather weak and less frustrated. The $\boldsymbol{b}$-component of $\boldsymbol{k}_{\mathrm{IC}}$ is therefore still commensurate. The overall magnetic frustration is especially apparent in measurements of the magnetic susceptibility where the Curie-Weiß-behavior at high temperatures yields frustration parameters of $f=4.25-6[37,47]$.

Furthermore, anisotropic thermal expansion due to magnetostriction was reported in all antiferromagnetic phases [48]. The unit cell shrinks continuously with decreasing temperature between $T_{\mathrm{N} 3}$ and $T_{\mathrm{N} 1}$ along the $\boldsymbol{a}$ - and $\boldsymbol{c}$-axis while it expands in $\boldsymbol{b}$-direction. Another step-like anomaly can be observed during the first order transition at $T_{\mathrm{N} 1}$ where a contraction of the $\boldsymbol{a}$ - and $\boldsymbol{b}$-axis takes place while this time $c$ is increased. The effect of magnetic ordering on the lattice can not only be observed by the dimensions of the unit cell, but also by a modulation of the crystal structure that follows the magnetic modulation. Superlattice reflections were found by $\mathrm{X}$-ray diffraction that contain no contribution of magnetic scattering and appear to be a higher order of the magnetic modulation vector: $\boldsymbol{k}_{\text {lat }}=2 \boldsymbol{k}_{\mathrm{mag}}$ [15]. The lattice modulation is therefore also commensurate in the AF1-phase and incommensurate in AF2 and AF3.

\subsubsection{Ferroelectricity}

The multiferroic nature of $\mathrm{MnWO}_{4}$ was independently discovered by Taniguchi [47] and Arkenbout [35] in the year 2006. Both groups found a sharp peak in the real part of the dielectric 
permittivity at $T_{\mathrm{N} 2}$ and a step-like anomaly at $T_{\mathrm{N} 1}$. Measurements of the spontaneous polarization confirmed that only the AF2-phase is ferroelectric with $P=60 \mu \mathrm{C} \mathrm{m}^{-2}$ along the $\boldsymbol{b}$-axis. The temperature-dependence of the ferroelectric hysteresis loop shows a maximum of the remanence polarization as well as the coercive field at $T=10 \mathrm{~K}$ [49].

Soon, striking discoveries about to the polarization were made. Using polarized neutron scattering, it is possible to determine the chirality $\boldsymbol{C}=\boldsymbol{S}_{\mathrm{i}} \times \boldsymbol{S}_{\mathrm{j}}$ of the spin-cycloid. It turned out that an external electric field can not only switch the direction of the polarization, but also changes the sign of the chirality [44]. Since the absolute values of both quantities are directly proportional, ferroelectric hysteresis loops can be determined with $C$ instead of $P$. This is not only evidence for the type-II multiferroic nature of $\mathrm{MnWO}_{4}$, but also an indicator that ferroelectricity is induced by $\mathrm{DMI}$ which can be described according to (for more details see chapter 2.2.1):

$$
P \propto k \times\left(S_{\mathrm{i}} \times S_{\mathrm{j}}\right)=k \times C
$$

$\boldsymbol{C}$ is perpendicular to both easy-axes and therefore lies in the $\boldsymbol{a c}$-plane. Since $\boldsymbol{k}$ also lies in the $\boldsymbol{a c}$-plane, $\boldsymbol{P}$ has to be parallel to the $\boldsymbol{b}$-axis, which is in accordance to experimental results. This approves DMI to be the origin of ferroelectricity in $\mathrm{MnWO}_{4}$. It is further supported by the fact that the direction of $\boldsymbol{P}$ can be reversed by an applied magnetic field [50].

$\mathrm{MnWO}_{4}$ also exhibits a pronounced memory-effect where the first poling with an electric field after cooling to the ferroelectric phase creates a preferred orientation for the polarization. If a sample is cooled down to the paralectric AF1-phase after poling and then heated up to AF2 again, the polarization has still the same sign and amplitude as before [35]. The orientation of ferroelectric domains is restored by cycling through AF1 although there are no ferroelectric domains present in the AF1-phase [51]. This effect is also observed when the AF1 $\rightarrow$ AF2 transition is induced by a magnetic field [50] (more about magnetic field induced phase transitions in chapter 2.3.1.4) and even when the sample is heated up slightly above $T_{\mathrm{N} 3}$ [52]. After reversing the direction of the polarization with an applied electric field and then cycling through AF1, $\boldsymbol{P}$ is again oriented along the first poling direction like the reversal never happened [52]. The aforementioned chirality hysteresis loops are asymmetric according to the preferred direction of $\boldsymbol{C}$. This asymmetry is more pronounced at temperatures close to the phase transitions, especially at $T_{\mathrm{N} 1}$ where it is not even possible any more to change the sign of $\boldsymbol{C}$ [44]. Furthermore, a stroboscopic polarized neutron scattering study investigated the time-dependence of domain switching with applied electric field and found that the reorientation of domains into the preferred direction is considerably faster than into the opposite direction [53]. 


\subsubsection{Phase transition by external magnetic field}

One of the most intriguing properties of multiferroics like $\mathrm{MnWO}_{4}$ is the possibility to induce phase transitions with an external magnetic field, especially since the electric properties differ from phase to phase. It is therefore possible, depending on the particular transition, to switch the spontaneous polarization on and off or to change its sign and direction, respectively. This discovery was a big step forward on the way to design magnetoelectric materials whose electric properties can be influenced by an external magnetic field.

The individual transitions strongly depend on the direction of the applied field. There are studies on $\mathrm{MnWO}_{4}$ with fields along the primary and secondary easy-axis as well as the hard-axis while different physical properties were measured like specific heat, magnetic susceptibility [42], dielectric permittivity, spontaneous polarization $[15,35,47,49,54]$ and sound velocity $[55]$.

When a magnetic field is applied along the primary easy-axis, it destabilizes the AF1- and AF3phase where all spins are parallel or antiparallel to the field. AF2 on the other hand, with spincomponents along the $\boldsymbol{b}$-axis perpendicular to the field, is stabilized and becomes the magnetic ground state above $2 \mathrm{~T}$ (see Figure 2-12, left) until a paraelectric high-field phase (called HF) emerges around $14 \mathrm{~T}[15,42]$. Applying a magnetic field of the same strength but at higher temperatures, it appears another phase AFIV which is ferroelectric with polarization along the $\boldsymbol{b}$-axis, but with opposite sign compared to AF2. AFIV becomes the magnetic ground state above $38 \mathrm{~T}$ until a final phase transition into the paraelectric AFV-phase takes place [54]. The magnetic structure of all three new phases is unknown. So, applying a magnetic field at $T=5 \mathrm{~K}$ yields following phase transitions (see also Figure 2-12, left):

$$
\operatorname{AF} 1(\boldsymbol{P}=0) \stackrel{2 \mathrm{~T}}{\longrightarrow} \mathrm{AF} 2(\boldsymbol{P} \| \boldsymbol{b}) \stackrel{14 \mathrm{~T}}{\longrightarrow} \mathrm{HF}(\boldsymbol{P}=0) \stackrel{38 \mathrm{~T}}{\longrightarrow} \operatorname{AFIV}(\boldsymbol{P} \|-\boldsymbol{b}) \stackrel{50 \mathrm{~T}}{\longrightarrow} \operatorname{AFV}(\boldsymbol{P}=0)
$$

At this temperature, it is possible to switch the polarization on, change its sign and switch it off, depending on the strength of the magnetic field.

The observation that phases with spin-components parallel to the applied field become unstable is also true when the field is applied along the secondary easy-axis. In this case, the AF2phase is destabilized [42]. The temperature range with spontaneous polarization becomes smaller with stronger fields (see Figure 2-12, right) while the amplitude of $\boldsymbol{P}_{\mathrm{s}}$ as well as the $b$-component of the spins is decreasing. The cycloid forms a highly asymmetric ellipse. At $10 \mathrm{~T}, \mathrm{MnWO}_{4}$ transitions from AF2 into another ferroelectric phase with an asymmetric cycloid perpendicular to the $\boldsymbol{b}$-axis $[56,57]$. This phase is called AF2' or X-phase and has the same modulation vector as AF2, but the 
polarization points along the $\boldsymbol{a}$-axis [47]. At $T=10.5 \mathrm{~K}$, it is therefore possible to induce a so called "spin-flop transition", changing the orientation of the polarization (see also Figure 2-12, right):

$$
\mathrm{AF} 2(\boldsymbol{P} \| \boldsymbol{b}) \stackrel{10.5 \mathrm{~T}}{\longrightarrow} \mathrm{AF}^{\prime} 2(\boldsymbol{P} \| \boldsymbol{a})
$$

A magnetic field along the hard-axis does not have any significant influence on the sequence of phase transitions [42].
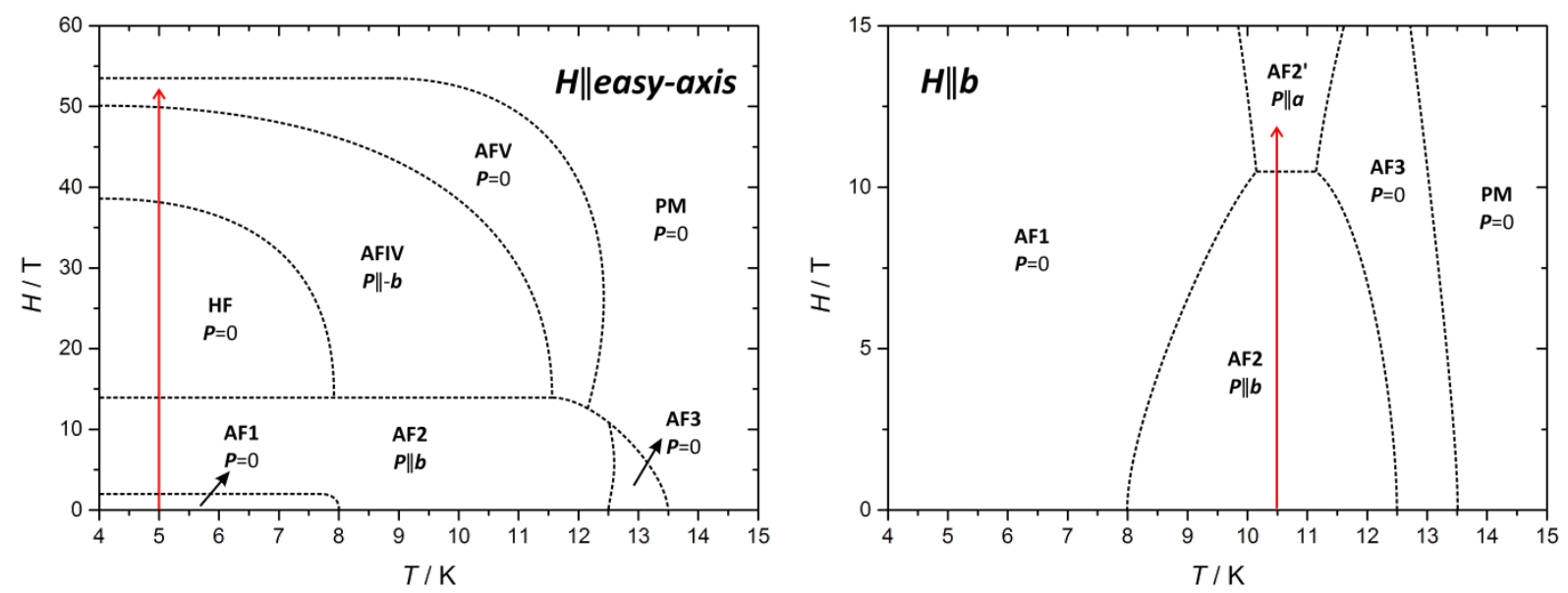

Figure 2-12: Schematic representation of the magnetic phase diagram of $\mathrm{MnWO}_{4}$ with magnetic field applied along the primary easy-axis (left) and along the secondary easy-axis (right), respectively. Red arrows are the sequences of phase transitions described in the text. Figures are inspired by [55,57]. 


\subsubsection{5 $\mathrm{MnW}_{1-\mathrm{x}} \mathrm{Mo}_{\mathrm{x}} \mathrm{O}_{4}$}

Chemical doping is another widely employed tool to investigate different properties of substances, but it can also be used to tailor materials according to own requirements. Since most type-II multiferroics have a highly frustrated spin order that is sensitive to small changes of the superexchange interactions, it is possible to modify the magnetic, and consequently the electronic, properties by small amounts of dopants. It is usually the magnetic atom that is replaced by another magnetic ion with different electron configuration or even by a diamagnetic ion. Another way is the substitution of ions with different radii that might induce lattice distortions and therefore indirectly alter superexchange.

$\mathrm{Mn}^{2+}$ in $\mathrm{MnWO}_{4}$ has so far been doped by a wide range of different elements and there are some interesting similarities in some cases but also striking differences in others. A few examples shall be mentioned at this point. There is a general tendency that $T_{\mathrm{N} 1}$ rapidly decreases with increased amount of dopant until AF2 becomes the new magnetic ground state. This is the case for $\mathrm{Cu}$ [58], Ni [59,60], Co [61], Mg and Zn [62]. This effect was already mentioned in chapter 2.3.1.2 and can also be observed for pure $\mathrm{MnWO}_{4}$ where the increased amount of grain boundaries in powder compared to single crystals decreases $T_{\mathrm{N} 1}$ and broadens the phase transition [38]. In contrast, Fedoping stabilizes the AF1-phase until AF2 completely vanishes with amounts of only $10 \% \mathrm{Fe}$. Applying a magnetic field of $5 \mathrm{~T}$ parallel to the primary easy-axis completely recovers AF2. The other two magnetic phase transition temperatures, $T_{\mathrm{N} 2}$ and $T_{\mathrm{N} 3}$, are linearly dependent on the degree of doping in most cases. They increase with $\mathrm{Cu}$-substitution [58] and decrease for $\mathrm{Ni}[59,60], \mathrm{Co}[61]$, $\mathrm{Mg}$ and $\mathrm{Zn}$ [62]. Furthermore, $10 \%$ of Co induces the appearance of a new magnetic phase which is absent in pure $\mathrm{MnWO}_{4}[61]$.

There are numerous studies that focus on the substitution of the magnetic $\mathrm{Mn}$-ion, but doping of the diamagnetic W-ion is rather rare. Until today, there is only a handful of publications about the system $\mathrm{MnW}_{1-\mathrm{x}} \mathrm{Mo}_{\mathrm{x}} \mathrm{O}_{4}$ where $\mathrm{W}^{6+}$ is replaced by the lighter equivalent $\mathrm{Mo}^{6+}$ that also has $d^{0}$-electron configuration and is diamagnetic $[18,63,64]$. The phase transition temperatures $T_{\mathrm{N} 2}$ and $T_{\mathrm{N} 3}$ increase linearly with $x$ (see Figure 2-13), making Mo-doping interesting as a small step on the path towards designing room-temperature multiferroics. The increase of magnetic phase transition temperatures is a result of stronger superexchange interactions which is in turn a consequence of the different electronic properties of molybdenum and tungsten, respectively. Since Mo has a lower electronegativity than $\mathrm{W}$ [65], Mo-O-bonds are more ionic than W-O-bonds. This has the effect that Mn-O-bonds, on the other hand, become more covalent with increased Mo-content, leading to stronger superexchange. There are no considerable distortions of the crystal structure expected by Mo-doping since the ionic radii of six-fold coordinated $\mathrm{Mo}^{6+}$ and $\mathrm{W}^{6+}$ are nearly identical $[66,67]$. 
Nevertheless, a slight linear decrease of the unit cell volume is observed [18] which could further strengthen superexchange.

The increase of superexchange interactions is not only evident by the phase transition temperatures, but also by the high-temperature behavior of the magnetic susceptibility. The extrapolated Curie-Weiß-temperature increases with Mo-content while the frustration parameter remains constant. The magnetic phase transition temperatures were determined by measurements of magnetic susceptibility [18,63,64], dielectric permittivity [18], electric polarization [64] and heat capacity [63]. $T_{\mathrm{N} 1}$ decreases drastically (see Figure 2-13) and the AF2 $\rightarrow$ AF1 transition is absent for $x \geq 0.1$. As previously mentioned, this effect is observed for a wide range of different dopants. A neutron diffraction study confirmed that the AF2-phase of an $x=0.2$ sample persists until at least $T=1.5 \mathrm{~K}$ [63]. The spontaneous polarization decreases with $x$ and could only be determined for $x \leq 0.1$ samples [64]. In accordance, the real part of the dielectric permittivity exhibits a peak at $T_{\mathrm{N} 2}$ whose height also decreases with $x$, but is still detectable at $x>0.1$. Interestingly, there appears another peak at the temperatures $T_{\mathrm{x}}$ which is linearly dependent of $x$ with nearly the same slope as $T_{\mathrm{N} 2}$ and $T_{\mathrm{N} 3}$ [18] (see Figure 2-13). When $T_{\mathrm{x}}$ is extrapolated to $\mathrm{x}=0$, it coincides with $T_{\mathrm{N} 1}$. This temperature is, however, neither connected to the AF2 $\rightarrow$ AF1 phase transition, the onset of polarization nor any change of magnetic ordering, but is also observed by Cu-doping [58].

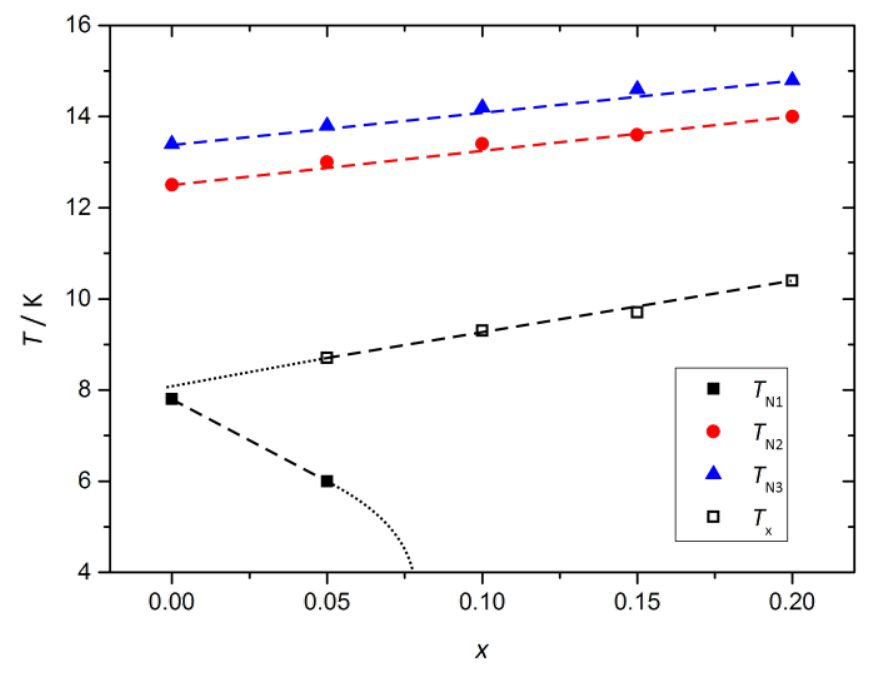

Figure 2-13: Influence of Mo-doping on the magnetic phase transition temperatures $\left(T_{\mathrm{N} 1}-T_{\mathrm{N} 3}\right)$ and the low-temperature peak in the real part of the dielectric permittivity $\left(T_{\mathrm{x}}\right)$. All lines are guides to the eye. This figure bases on data published in [63].

Although there are some studies about $\mathrm{MnW}_{1-x} \mathrm{Mo}_{x} \mathrm{O}_{4}$, the maximum possible amount of Mo-doping still remains ambiguous. It is not possible to completely replace $\mathrm{Mn}$ by Mo because the room-temperature crystal structure of $\mathrm{MnMoO}_{4}$ is considerably different than $\mathrm{MnWO}_{4}$. Both compounds crystallize in the space group $P 2 / c$, but $\mathrm{W}$ is octahedrally coordinated in $\mathrm{MnWO}_{4}$ while 
the oxygen atoms around $\mathrm{Mo}$ in $\mathrm{MnMoO}_{4}$ form tetrahedra that are not connected to each other (see Figure 2-14) [68]. The Mo-O-bonds are shorter than in Mo-doped $\mathrm{MnWO}_{4}$ which is accordance with the ionic radius that is smaller for the four-fold coordinated $\mathrm{Mo}^{6+}$ compared to the six-fold equivalent $[66,67]$. The system $\mathrm{MnW}_{1-\mathrm{x}} \mathrm{Mo}_{x} \mathrm{O}_{4}$ therefore exhibits a miscibility gap that was already investigated decades ago. It was found that $10 \% \mathrm{Mo}$ can be solved in $\mathrm{MnWO}_{4}$ at $600^{\circ} \mathrm{C}$ while this value increases up to $15 \%$ at $1100^{\circ} \mathrm{C}$ [69]. This is in contrast to recent studies where samples of $x=0.3$ could be synthesized that did not show any sign of a $\mathrm{MnMoO}_{4}$-phase [18]. These opposing results might be explained by differences in the synthesis methods [70]. Another study claims that it is even possible to solve up to $50 \%$ molybdenum by extensive ball milling after synthesis [71].

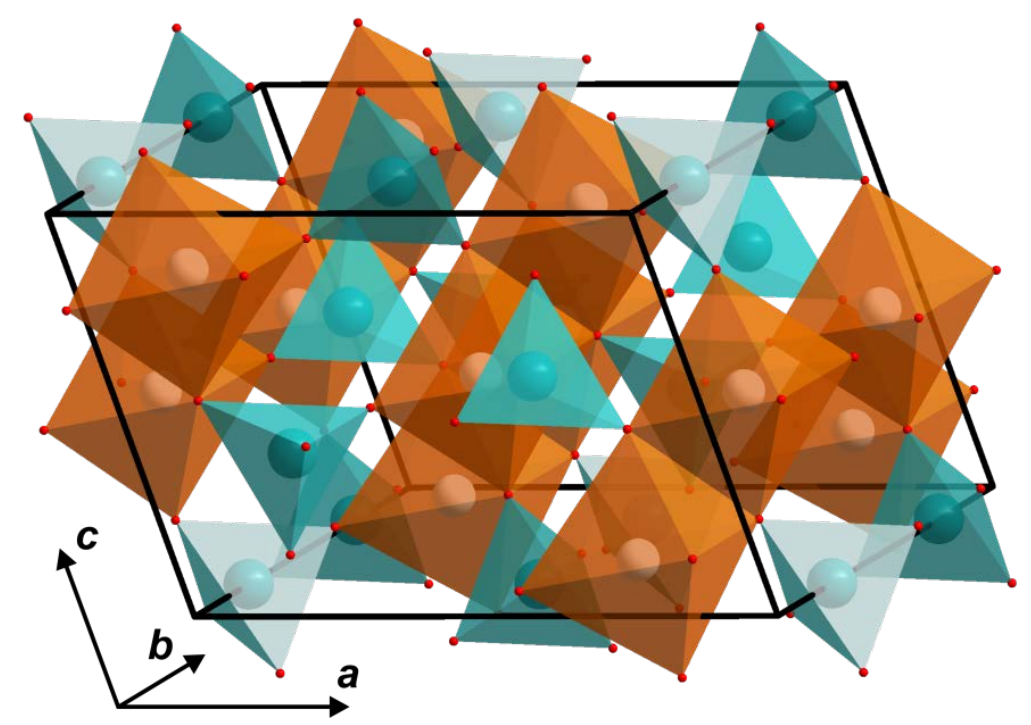

Figure 2-14: Crystal structure of $\mathrm{MnMoO}_{4}$. White spheres are $\mathrm{Mn}$-ions octahedrally surrounded by oxygen atoms (red spheres) as indicated by orange color. Mo-ions are represented by green spheres with tetrahedral coordination geometry as indicated by green color. Crystallographic data for this figure are taken from [68]. 


\subsubsection{CuO}

$\mathrm{CuO}$ has a rather simple chemical composition, but it nevertheless attracted a lot of attention during $80 \mathrm{~s}$ and $90 \mathrm{~s}$ of the last century as model substance for high-temperature superconductors. The interest faded away after a while, but since the discovery of spontaneous electric polarization in the antiferromagnetic phase, $\mathrm{CuO}$ became again a focus of research as model substance for hightemperature multiferroics.

Although $\mathrm{CuO}$ has a completely different chemical and crystal structure than $\mathrm{MnWO}_{4}$, both materials share several common features like magnetic structure, sequence of phase transitions and origin of ferroelectricity. The next subchapters will therefore mainly focus on properties unique to $\mathrm{CuO}$ while all similarities will be only briefly addressed since they have been discussed in the previous chapter.

\subsubsection{Crystal structure}

Like $\mathrm{MnWO}_{4}, \mathrm{CuO}$ crystallizes in the monoclinic space group P2/c [72] with lattice parameters $a=4.6837(5), b=3.4226(5), c=5.1288(6), \beta=99.54(1)^{\circ}$ [73]. There are four chemical formula units per crystallographic unit cell. The relative coordinates of all atoms of the asymmetric unit cell are listed in Table 2-3. Each oxygen atom is surrounded by four $\mathrm{Cu}$-atoms, forming a distorted tetrahedron. $\mathrm{Cu}^{2+}$ has $3 d^{9}$ electron configuration with total spin of $S=0.5$ and is therefore prone to Jahn-Teller- displacement. This is reflected by a highly distorted octahedral coordination geometry of surrounding oxygen ions where the average bond length in the basal plane is considerably smaller $(\approx 1.96 \AA)$ than the distance to apical oxygen atoms $(2.79 \AA)$. The apical bond is also tilted by $17^{\circ}$ with respect to the normal vector of the basal plane. The coordination can rather be described as rectangles of oxygen atoms that form infinite edge shared chains (see Figure 2-15, left). There are two types of chains propagating in different directions, [ 110$]$ and $\left[\begin{array}{lll}1 & -1 & 0\end{array}\right]$, respectively. The direction alternates along the normal vector of the $\boldsymbol{a b}$-plane while each chain is corner-connected to its neighboring chains.

The bonding between neighboring $\mathrm{Cu}$-atoms is mediated via oxygen atoms, forming infinite ...-Cu-O-Cu-O-... zig-zag chains in [ $\left[\begin{array}{lll}1 & 0 & 1\end{array}\right]$ - and $\left[\begin{array}{lll}1 & 0 & -1\end{array}\right]$-direction, respectively. The Cu-O-Cu-bond angles vary significantly for both types of chains, with $108.5^{\circ}$ along [ 10101 and $146.2^{\circ}$ along [ $\left[\begin{array}{lll}1 & 0 & -1\end{array}\right]$. Both types of bonds are represented by yellow and red tubes, respectively, in Figure 2-15, right. Every $\mathrm{Cu}$-atom is part of one chain of each type. 
Table 2-3: Relative coordinates of all atoms of the asymmetric unit cell of CuO [73].

\begin{tabular}{|c|c|c|c|}
\hline & $\boldsymbol{x}$ & $\boldsymbol{y}$ & $\boldsymbol{z}$ \\
\hline $\mathrm{Cu}$ & 0.25 & 0.25 & 0 \\
\hline $\mathrm{O}$ & 0 & $0.4163(7)$ & 0.25 \\
\hline
\end{tabular}
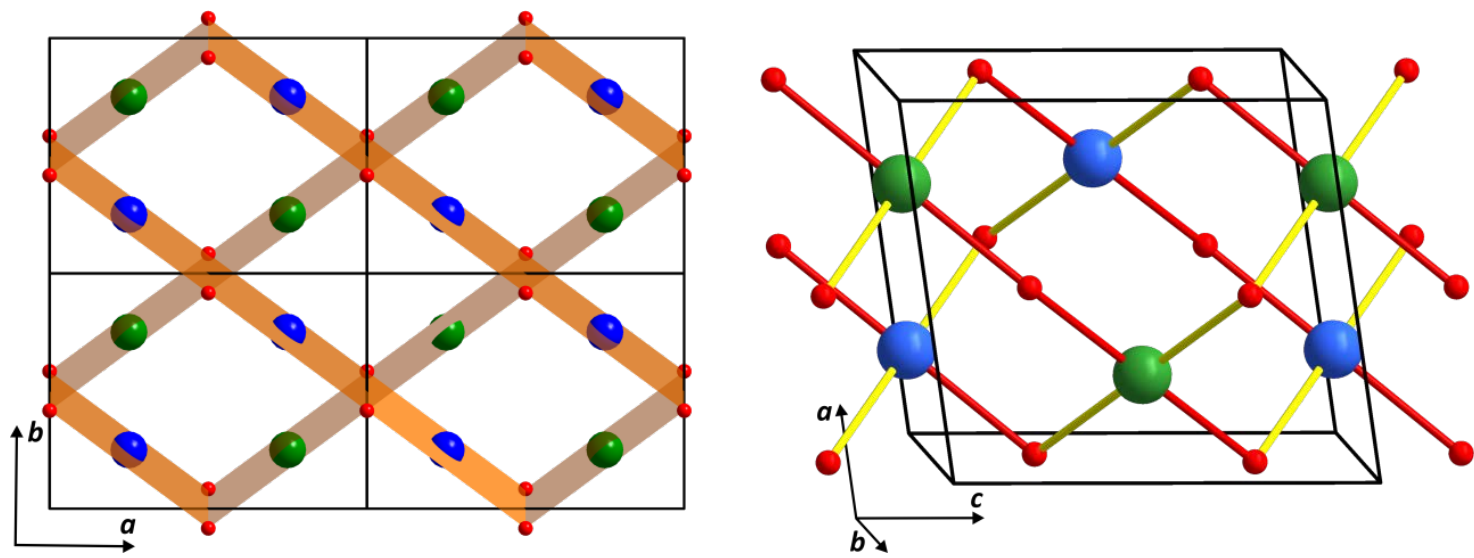

Figure 2-15: Crystal structure of $\mathrm{CuO}$ with view perpendicular to the $\boldsymbol{a} \boldsymbol{b}$-plane (left) and approximately along the $\boldsymbol{b}$-axis (right). Blue and green spheres are $\mathrm{Cu}^{2+}$-ions with different relative coordinates. In the left panel, green and blue $\mathrm{Cu}$-ions have $\mathrm{z}=0,1$ and $z=0.5$, respectively. In the right panel, green and blue Cu-ions have $y=0.25$ and 0.75 , respectively. Red spheres are oxygen atoms. Orange planes in the left panel represent oxygen coordination rectangles. Yellow and red tubes in the right panel represent chains of Cu-O-Cu-bonds in [ $\left[\begin{array}{lll}1 & 0 & 1\end{array}\right]$ - and [ $\left[\begin{array}{lll}1 & 0 & -1\end{array}\right]$-direction, respectively. Crystallographic data for this figure are taken from [73].

\subsubsection{Magnetic structure}

Brockhouse first determined the antiferromagnetic modulation of $\mathrm{CuO}$ more than 60 years ago [74]. Afterwards it took more than 30 years until the magnetic structure of at least two out of three phases was finally solved [75-78]. In fact, there are three successive antiferromagnetic phase transitions at temperatures $T_{\mathrm{N} 1}=213 \mathrm{~K}[19,79-81], T_{\mathrm{N} 2}=229.2 \mathrm{~K}$ and $T_{\mathrm{N} 3}=229.8 \mathrm{~K}[82,83]$. These temperatures are considerably higher than in most other type-II multiferroics, like for example $\mathrm{MnWO}_{4}$. This makes $\mathrm{CuO}$ quite unique as a promising stepping stone in the search for roomtemperature multiferroics. Like $\mathrm{MnWO}_{4}$, the transition at $T_{\mathrm{N} 1}$ is of first order nature $[80,83]$ while second order transitions take place at $T_{\mathrm{N} 2}$ and $T_{\mathrm{N} 3}$ [81,83]. Unlike $\mathrm{MnWO}_{4}$, the primary easy-axis coincides with the $\boldsymbol{b}$-axis $[75,76]$ while the secondary easy-axis lies in the $\boldsymbol{a c}$-plane with an angle of $28.2^{\circ}$ towards the $c$-axis $[76,84]$. The latter can be expressed in terms of reciprocal lattice vectors as $0.506 a^{*}+1.517 c^{*}$.

In the antiferromagnetic low-temperature phase AF1, all spins are either parallel or antiparallel to the $\boldsymbol{b}$-axis as represented in the left panel of Figure $2-16$. The commensurate modulation is 
described by $\boldsymbol{k}_{\mathrm{C}}=(0.5,0,-0.5)[74,84]$ which results in a perfect ferromagnetic alignment of spins along the $\left[\begin{array}{lll}1 & 0 & 1\end{array}\right]$-direction.

The AF2-phase between $T_{\mathrm{N} 1}$ and $T_{\mathrm{N} 2}$ exhibits a circular [77] cycloidal order where all spins lie in a plane spanned by both easy-axes with an incommensurate modulation according to the temperature-independent vector $\boldsymbol{k}_{\mathrm{IC}}=(0.506,0,-0.483)$ [75,84]. The spins of Cu-atoms in [1 01$]$ direction are in this phase no longer parallel, but experience a slight phase shift which leads to a rotation in the cycloidal plane. This is indicated in the right panel of Figure 2-16 by a small shift of the "spin up"- and "spin down"-symbols and by a shortening of the blue arrow, respectively.

The magnetic structure of the AF3-phase has not been determined, yet. Only the phase transition temperatures were measured so far by dilatometry [83] and ultra sound [82]. In analogy to $\mathrm{MnWO}_{4}$, it is speculated that the spins are collinearly oriented along the $\boldsymbol{b}$-axis with an incommensurate modulation of the amplitude according to $\boldsymbol{k}_{\mathrm{IC}}$ [82].

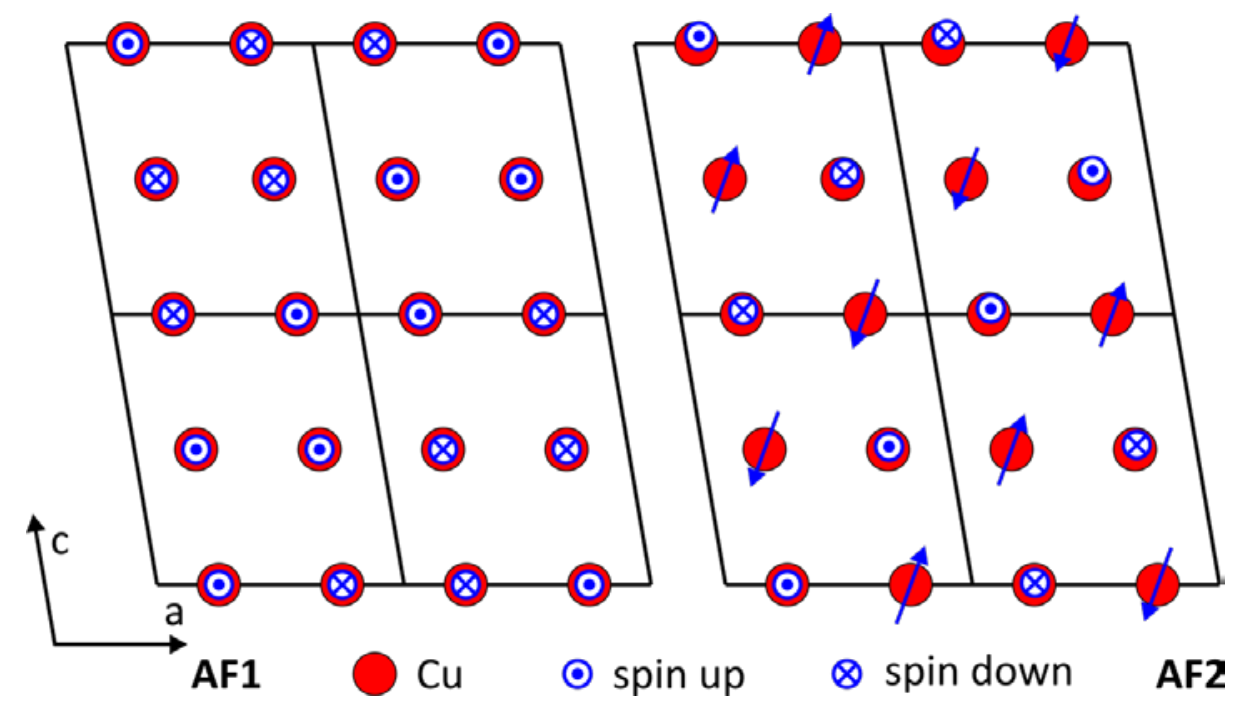

Figure 2-16: Schematic representation of the magnetic structure of $\mathrm{CuO}$ in the phases AF1 (left) and AF2 (right), viewing along the $\boldsymbol{b}$-axis. Red spheres are $\mathrm{Cu}^{2+}$-ions and their according spins are represented as blue arrows. Please note in the right panel, that the slight rotation of spins due to the incommensurate modulation is indicated by a small shift of the "spin up"- and "spin down"-symbols and by a shortening of the blue arrow, respectively. Crystallographic data for this figure are taken from [73].

Like $\mathrm{MnWO}_{4}, \mathrm{CuO}$ exhibits anisotropic magnetostriction at the magnetic phase transitions [83], although it is less pronounced. There is a clear contraction of the $\boldsymbol{a}$-axis and an expansion of the $\boldsymbol{b}$-axis at $T_{\mathrm{N} 1}$ while the changes at $T_{\mathrm{N} 2}$ and $T_{\mathrm{N} 3}$ are more subtle. The expansion coefficient shows a maximum in $\boldsymbol{a}$ - and $\boldsymbol{c}$-direction at $T_{\mathrm{N} 2}$ and a minimum along the $\boldsymbol{b}$-axis. The anomaly at $T_{\mathrm{N} 3}$, however, is only manifested in a change of the second temperature derivative of the lattice paramters.

Another peculiarity of $\mathrm{CuO}$ is a broad peak in the magnetic susceptibility reaching from 400 to $800 \mathrm{~K}$ with the maximum around $540 \mathrm{~K}$, well above the magnetic ordered phase at $T_{\mathrm{N} 3}$. This is 
explained by a model called "molecular antiferromagnetismus" $[79,85]$. Instead of a long-range order of the whole crystal, only two neighboring Cu-ions form a dimer with an antiferromagnetic ground state $(S=0)$ and a ferromagnetic excited state $(S=1)$. Short-range ordering above $T_{\mathrm{N}}$ is a common feature of frustrated magnets and also $\mathrm{MnWO}_{4}$ shows slight deviations from the high-temperature Curie-Weiß-behavior at temperatures six times higher than $T_{\mathrm{N}}$, as explained in chapter 2.3.1.2. The magnetic short-range interactions in $\mathrm{CuO}$, however, are rather extreme since there is no Curie-Weiß behavior at all.

The reason for this is a strong antiferromagnetic superexchange integral mediated by $\mathrm{Cu}-\mathrm{O}-\mathrm{Cu}$ bonds along the [1 $0-1]$-direction, $J_{[10-1]} \approx 91 \mathrm{meV}$, which is much stronger than all other relevant interactions [86-88]. This is in itself a big difference to, for example, $\mathrm{MnWO}_{4}$ where the exchange integrals also vary over three orders of magnitude, but the strongest three interactions are equal in strength $(\approx 0.5 \mathrm{meV})$ as well as along different directions [32,46]. The magnetic structure of $\mathrm{MnWO}_{4}$ is therefore rather three-dimensional while the structure of $\mathrm{CuO}$ in the AF1-phase can be regarded as one-dimensional antiferromagnetic chains, propagating along [10-1]. The structure is further supported by ferromagnetic exchange integrals in $\left[\begin{array}{lll}1 & 0 & 1\end{array}\right]$-direction and along the $\boldsymbol{b}$-axis which are in turn one and two orders of magnitude weaker, respectively [88]. Magnetic frustration in CuO only

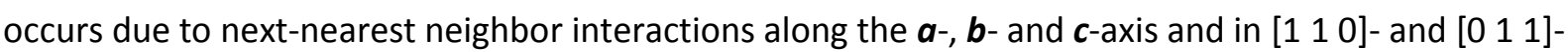
direction, respectively, which are all much weaker than $J_{[10-1]}$.

The magnitude of $J_{[10-1]}$ as well as the weak frustration explain the comparatively high phase transition temperatures of $\mathrm{CuO}$. It is also reflected by steep magnon dispersion curves close to the magnetic $\Gamma$-point $[86,89,90]$ and the high-energy magnetic excitation spectrum that has the shape of a one-dimensional $S=0.5$ antiferromagnet $[87,88]$.

\subsubsection{Ferroelectricity}

Spontaneous electric polarization in CuO was first discovered by Kimura in 2008 [19]. Like in $\mathrm{MnWO}_{4}$, it only appears in the AF2-phase with a magnitude of $P=160 \mu \mathrm{C} \mathrm{m}^{-2}$ along the $\boldsymbol{b}$-axis. There is a peak in the real part of the dielectric permittivity at $T_{\mathrm{N} 2}$ and a step-like anomaly at $T_{\mathrm{N} 1}[19,81]$. It is also possible to switch the cycloid chirality $C$ by an external electric field [91], as described for $\mathrm{MnWO}_{4}$ in chapter 2.3.1.3. Since both, $\boldsymbol{C}$ and the cycloid propagation vector $\boldsymbol{k}$, lie in the $\boldsymbol{a c}$-plane, the spontaneous polarization has to be parallel to the $\boldsymbol{b}$-axis, according to equation (2-12), which is accordance with experimental results. It is therefore safe to assume that ferroelectricity in $\mathrm{CuO}$ is induced by DMI. 
There are also magnetic field induced phase transitions reported for $\mathrm{CuO}$, but the magnetic phase diagrams are much less diverse than in the case of $\mathrm{MnWO}_{4}$ [92]. It can be observed that phases with spin components parallel to the field are destabilized. A magnetic field perpendicular to the $\boldsymbol{b}$-axis therefore favors the AF1- and AF3-phase. AF2 completely vanishes above $50 \mathrm{~T}$. A field parallel to the $\boldsymbol{b}$-axis, on the other hand, destabilizes AF1 and AF3 and induces phase transitions at $15 \mathrm{~T}$ into the high-field phases HF1 and HF3 with collinear and sinoidal spin order, respectively, perpendicular to the $\boldsymbol{b}$-axis. AF2 is also destabilized and vanishes, again, at $45 \mathrm{~T}$. In contrast to $\mathrm{MnWO}_{4}$, there are less high-field phases and none of them exhibits ferroelectricity. 


\subsection{3 $\quad \mathrm{Bi}_{2} \mathrm{Mn}_{4} \mathrm{O}_{10}$}

$\mathrm{Bi}_{2} \mathrm{Mn}_{4} \mathrm{O}_{10}$ (BMO) is a member of the $\mathrm{R}_{2} \mathrm{Mn}_{4} \mathrm{O}_{10}$-family with mullite-like crystal structure which is considerably more complex than $\mathrm{MnWO}_{4}$ and $\mathrm{CuO}$. $\mathrm{BMO}$ has not only two magnetic manganese ions with different oxidation states and coordination geometries, but also the overall magnetic structure, the sequence of phase transitions and the origin of ferroelectricity is different. All these properties will be discussed in the following subchapters.

\subsubsection{Crystal structure}

Since half a century, it is known $[93,94]$ that mullite-type BMO crystallizes in the orthorhombic space group Pbam with room-temperature lattice parameters of $a=7.5472(7) \AA, b=8.524$ (7) $\AA$ and $c=5.7836(5) \AA[95]$. The relative coordinates of all atoms of the asymmetric unit cell are listed in Table 2-4. There are two crystallographic sites of manganese atoms with different oxidation state and coordination geometry $[96,97] . \mathrm{Mn}^{4+}$ has a $d^{3}$ electron configuration with a total spin of $S=3 / 2$ and is octahedrally coordinated by oxygen atoms. The additional $d$-electron of $\mathrm{Mn}^{3+}$, on the other hand, leads to a $d^{4}$-high spin configuration with $S=2$ which is prone to Jahn-Teller distortion and therefore square pyramidally coordinated. The $\mathrm{Mn}^{4+}$-octahedra form infinite, edge-shared chains running along the $\boldsymbol{c}$-axis which are interconnected by dimers of $\mathrm{Mn}^{3+}$-pyramids in $\boldsymbol{a}$ - as well as $\boldsymbol{b}$-direction as displayed in Figure $2-17$, right. The basal planes of both pyramids share a common edge while all remaining oxygen atoms in this plane are at the same time a corner of different $\mathrm{Mn}^{4+}-$ octahedra. The apical oxygen atoms of both pyramids are pointing in opposite directions roughly along the $\boldsymbol{a}$-axis. This creates a rigid link between the chains along $\boldsymbol{b}$ and a rather lose connection along $\boldsymbol{a}$ that is reflected by the anisotropy of thermal expansion [95] and compressibility $[98,99]$ at room temperature which are both stronger in $\boldsymbol{a}$-direction.

The remaining voids between octahedra and pyramids form infinite channels along the $c$-axis. They are filled by bismuth ions which have eight oxygen atoms in their proximity but without a clear geometrical arrangement. Compared to other members of the $\mathrm{R}_{2} \mathrm{Mn}_{4} \mathrm{O}_{10}$ family, this void is quite distorted due to the lone electron pair of the $\mathrm{Bi}^{3+}$-ion with $s^{2} d^{0}$ electron configuration. Although the steric effect is smaller than in similar compounds like, for example, $\mathrm{Bi}_{2} \mathrm{Mn}_{4} \mathrm{O}_{9}$ [100], it is still responsible for a significant deformation of the $\mathrm{Mn}^{4+}$-octahedra and $\mathrm{Mn}^{3+}$-pyramids [101], creating localized, alternating dipoles along the $\boldsymbol{c}$ - and $\boldsymbol{b}$-direction [36], respectively.

The crystal structure of BMO can also be interpreted as layers perpendicular to the $c$-axis consisting of different metal ions with the sequence ...- $-\mathrm{Mn}^{4+}-\mathrm{Mn}^{3+}-\mathrm{Mn}^{4+}-\mathrm{Bi}^{3+}-\mathrm{Mn}^{4+}-\ldots$ (Figure 2-17, left). 

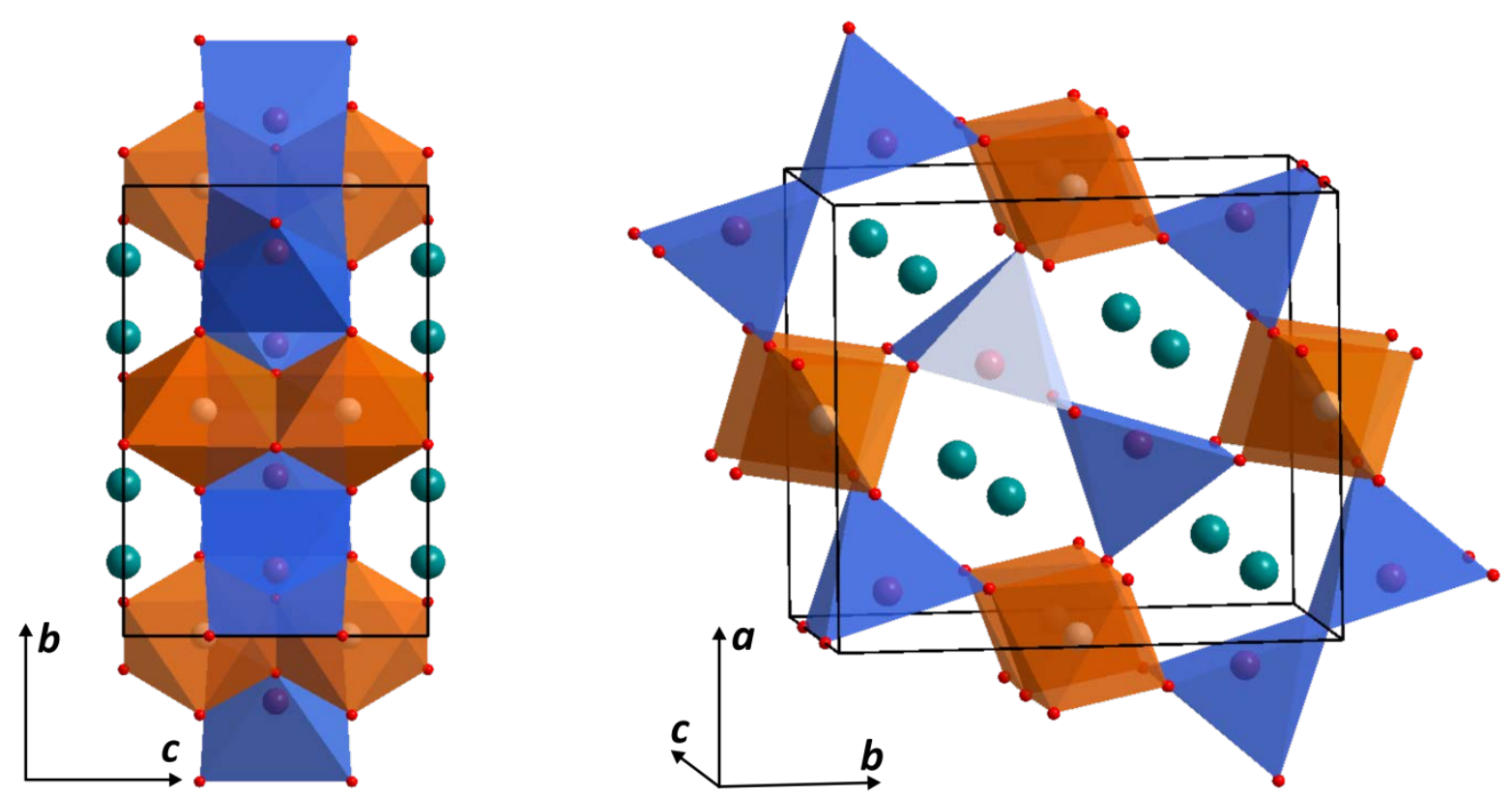

Figure 2-17: Crystal structure of $\mathrm{BMO}$, view along the $\boldsymbol{a}$-axis (left) and approximately the $\boldsymbol{c}$-axis (right). White spheres are $\mathrm{Mn}^{4+}$-ions which are octahedrally coordinated by oxygen atoms (small red spheres) as indicated by orange color. Big purple spheres are $\mathrm{Mn}^{3+}$-ions which have square pyramidal coordination geometry (indicated as blue) due to Jahn-Teller-distortion. The remaining green spheres are bismuth atoms. Crystallographic data for this figure are taken from [94].

Table 2-4: Relative coordinates of all atoms of the asymmetric unit cell of BMO [94].

\begin{tabular}{|c|c|c|c|}
\hline & $\boldsymbol{x}$ & $\boldsymbol{y}$ & $\boldsymbol{z}$ \\
\hline $\mathrm{Bi}$ & $0.1597(3)$ & $0.1643(3)$ & 0 \\
\hline $\mathrm{Mn}^{4+}$ & 0.5 & 0 & $0.262(3)$ \\
\hline $\mathrm{Mn}^{3+}$ & $0.407(1)$ & $0.354(1)$ & 0.5 \\
\hline $\mathrm{O}_{1}$ & 0 & 0 & $0.281(10)$ \\
\hline $\mathrm{O}_{2}$ & $0.386(4)$ & $0.176(4)$ & $0.250(8)$ \\
\hline $\mathrm{O}_{3}$ & $0.147(6)$ & $0.418(5)$ & 0.5 \\
\hline $\mathrm{O}_{4}$ & $0.147(6)$ & $0.425(5)$ & 0 \\
\hline
\end{tabular}

\subsubsection{Magnetic structure}

Long-range antiferromagnetic ordering in $\mathrm{BMO}$ is present in a single phase at temperatures below $T_{\mathrm{N}}=39 \mathrm{~K}$ with a commensurate modulation of $\boldsymbol{k}=(0.5,0,0.5)$ [97]. This is in contrast to other members of the $\mathrm{R}_{2} \mathrm{Mn}_{4} \mathrm{O}_{10}$-family where several transitions between commensurate and incommensurate phases are observed [102]. Figure 2-18 shows the magnetic structure which is dominated by the strong magnetic single-ion-anisotropy of the $\mathrm{Mn}^{3+}$-pyramids [103]. The preferred orientation of the spins is perpendicular to the basal plane, creating a nearly collinear ordering mainly along the $\boldsymbol{a}$-axis with only minor $\boldsymbol{b}$ - and $\boldsymbol{c}$-components. 
There are in total five relevant superexchange integrals that have to be considered [21]. Their magnitudes have not yet been determined experimentally, but some conclusions can be drawn from the magnetic structure. $\mathrm{Mn}^{3+}$-ions interact with each other via the edges of the basal planes $\left(J_{5}\right)$ while superexchange between $\mathrm{Mn}^{3+}$ and $\mathrm{Mn}^{4+}$ can take two different pathways, along the apical oxygen atom $\left(J_{4}\right)$ and along the pyramid base $\left(J_{3}\right)$ as displayed in the left panel of Figure 2-18. There are also two different interactions for the $\mathrm{Mn}^{4+}$-ions along the octahedra chains in $c$-direction depending on the intermediate atom. $J_{1}$ corresponds to layers that are separated by $\mathrm{Bi}$-atoms while the interaction between the two $\mathrm{Mn}^{4+}$ next to a $\mathrm{Mn}^{3+}$-pyramid is assigned to $J_{2}$ (see Figure 2-18, right panel). The strongest superexchange interactions can be found in $\boldsymbol{a}$-direction which leads to antiferromagnetic zig-zag-chains as indicated by a black dashed line in Figure 2-18 (left). This chain corresponds to the interactions $J_{4}$ and $J_{5}$ while the pathway in $\boldsymbol{b}$-direction along the basal planes involves $J_{3}$ and $J_{5}$ (blue dashed line in Figure 2-18 (left)). The latter is magnetically frustrated since there are two pairs of $\mathrm{Mn}^{3+}-\mathrm{Mn}^{4+}$-ions, one of which is parallel aligned and the other one antiparallel. No matter the sign and strength of $J_{3}$, one pair has the wrong orientation and the contribution of both pairs to the total free energy adds to zero [21]. Obviously, $J_{4}$ has to be stronger than $J_{3}$ because the antiferromagnetic chains would otherwise run along the $\boldsymbol{b}$-axis and the magnetic frustration would be observed in $\boldsymbol{a}$-direction. Also please note that there are two antiferromagnetic zig-zag chains per crystallographic unit cell [104], overlapping at the $\mathrm{Mn}^{3+}$-pyramids as shown in Figure 2-18 (right).

The modulation of spins on $\mathrm{Mn}^{4+}$-sites along the $c$-direction depends on the type of intermediate metal layer as displayed in Figure 2-19 where each panel only shows spins of ions with
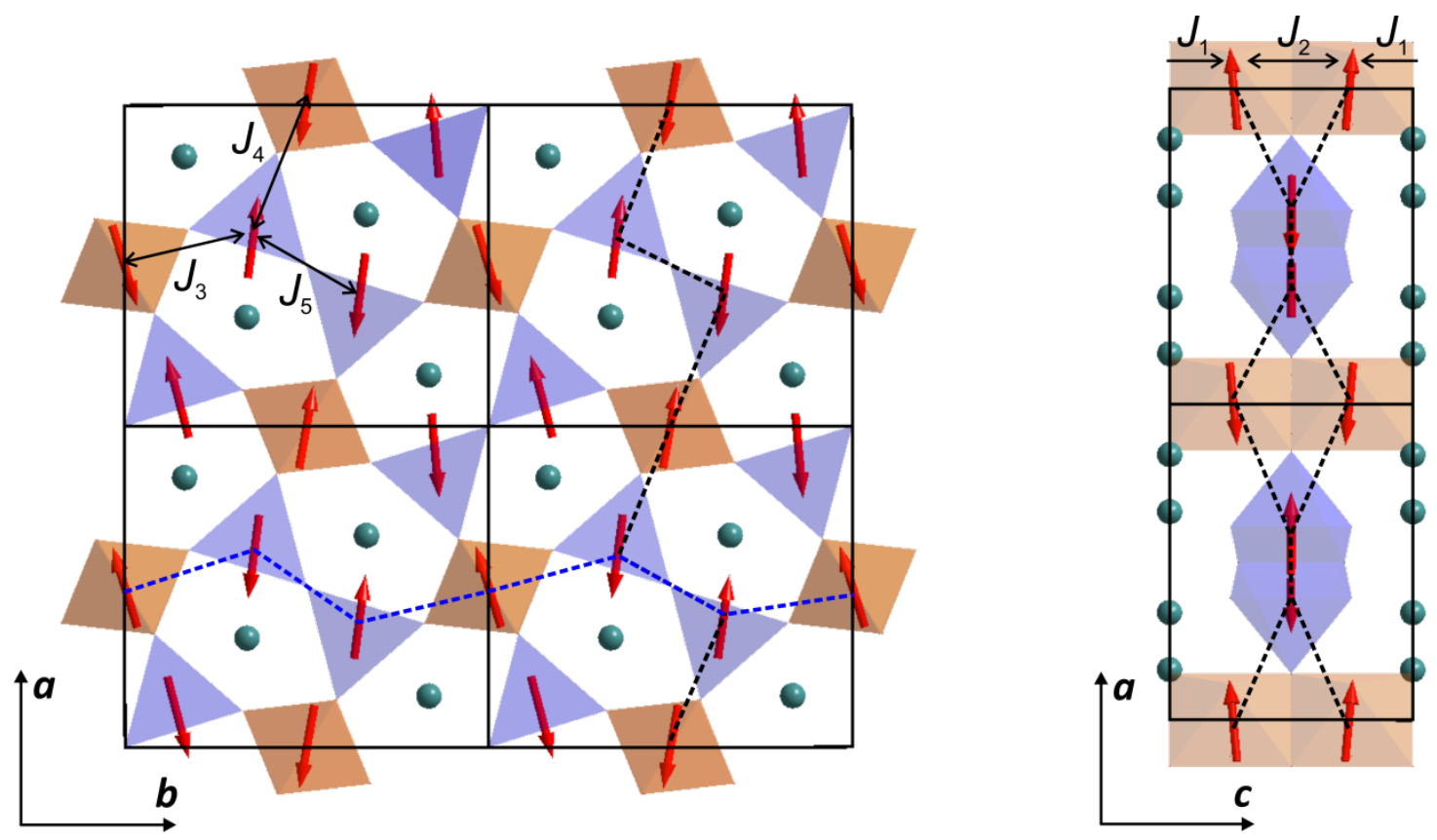

Figure 2-18: Magnetic structure of BMO viewing along the $c$-axis (left) and view on the antiferromagnetic zig-zag chains along the $\boldsymbol{b}$-direction (right). $\mathrm{Mn}^{4+}$-octahedra have orange color while $\mathrm{Mn}^{3+}$-pyramids are colored blue. Green spheres are nonmagnetic Bi-ions. Crystallographic data for this figure are taken from [94,103]. 
the same relative $c$-coordinate. The spins of the first two panels belong to $\mathrm{Mn}^{4+}$-ions which are separated by a layer of Bi-atoms which carry no spins and cannot contribute to the total free energy by any magnetic interaction. Hence, there are only direct superexchange interactions $\left(J_{1}\right)$ along the $\boldsymbol{c}$-axis between $\mathrm{Mn}^{4+}$-atoms of both layers, creating a perfect antiferromagnetic alignment. This is different for $\mathrm{Mn}^{4+}$-layers that are separated by $\mathrm{Mn}^{3+}$-pyramids (compare panel 2 and 4 of Figure 2-19) where the dominant interaction takes place inside the antiferromagnetic chains between $\mathrm{Mn}^{4+}$ - and $\mathrm{Mn}^{3+}$-layers, respectively. These indirect interactions between both $\mathrm{Mn}^{4+}$-layers via $J_{3}$ and $J_{4}$ are stronger than the direct superexchange $J_{2}$ and therefore favor a ferromagnetic alignment.

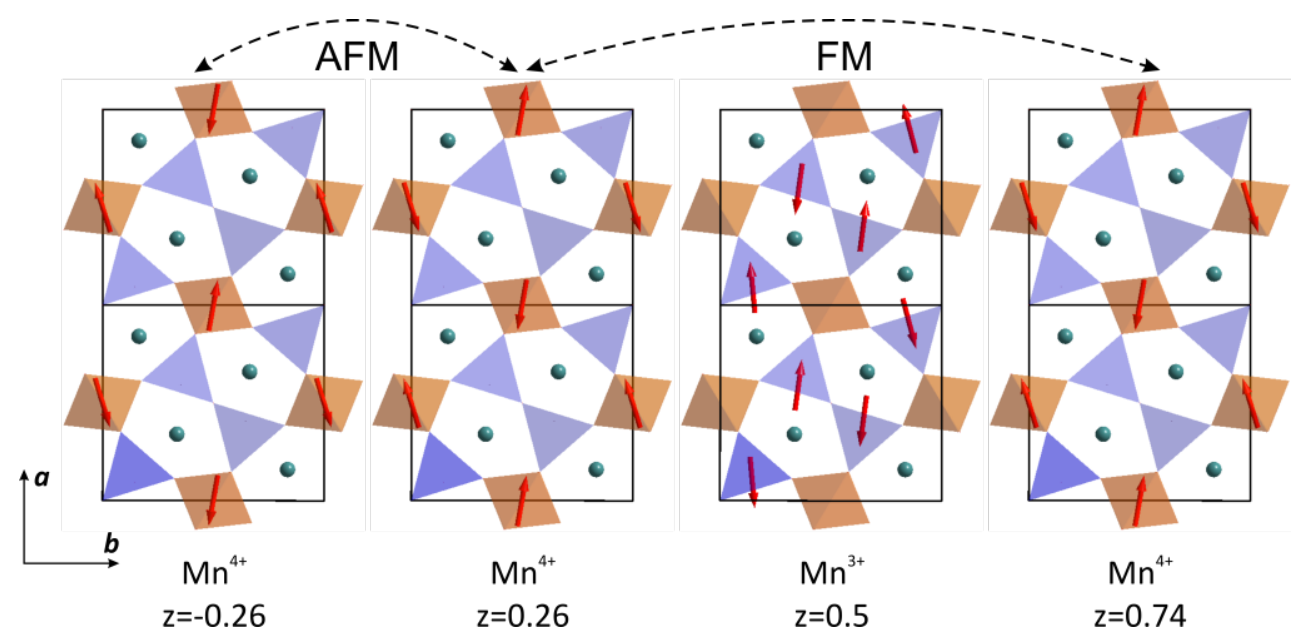

Figure 2-19: Modulation of spins along the $c$-direction. Each panel only shows spins of ions positioned at the relative $c$-coordinate indicated below the panel. This figure is inspired by [97] using crystallographic data from $[94,103]$.

Magnetic ordering also affects the lattice parameters of BMO below $T_{N}$ [105]. There is an expansion in $\boldsymbol{c}$-direction and an even stronger contraction along the $\boldsymbol{a}$-axis which leads to an overall reduction of the cell volume in the magnetic ordered phase that is distinct from usual anharmonic effects. The volume changes are proportional to the squared sublattice magnetization and can therefore be attributed to magnetostriction. Simultaneously, a broadening of Bragg reflections with finite $\boldsymbol{a}$-component was observed by $\mathrm{X}$-ray diffraction and is attributed to a modulation of bond length in this direction, also induced by magnetostriction [105]. Both effects can already be observed to a certain amount at temperatures slightly above $T_{\mathrm{N}}$. This is in accordance to the observation of anomalies in the magnetic susceptibility at temperatures around $T^{*}=60 \mathrm{~K}$ [101] that are accompanied by shifts of phonon frequencies measured by Raman spectroscopy [106]. This is a clear indication of spin-phonon-coupling induced by magnetic short range ordering. While these magnetostrictive effects are only observed slightly above $T_{N}$, there are already deviations of the magnetic susceptibility from the Curie-Weiß-behavior at considerably higher temperatures. Different frustration parameters are reported ranging from 6.3 to 7.5 [101,106,107]. 


\subsubsection{Ferroelectricity}

Up to this day, there are no reports of spontaneous polarization in the magnetically ordered phase of BMO and no dielectric investigation could find any evidence for a peak of the real permittivity at $T_{\mathrm{N}}[101,108-110]$, apart from the measurements presented in this thesis which can be found in chapter 6.3.1. So, all considerations about multiferroicity of BMO remain rather speculative, but there are some solid indicators that ferroelectricity indeed exists. The obvious reason is the similarity of crystal and magnetic structure compared to other members of the $\mathrm{R}_{2} \mathrm{Mn}_{4} \mathrm{O}_{10}$-family where polarization was experimentally observed [111]. Most of them exhibit a sequence of transitions between commensurate and incommensurate magnetic phases where ferroelectricity is usually observed when the modulation vector $\boldsymbol{k}$ has a commensurate $a$-component of 0.5 [102]. This is also the case in the antiferromagnetic phase of BMO. Furthermore, neutron diffraction studies revealed that the $c$-component of the magnetic moments on $\mathrm{Mn}^{4+}$-sites have an anti-phase relation to the remaining two elements of the spin vector $[102,103]$. This indicates that the overall magnetic structure has a minor cycloidal component as a result of the broken spatial inversion symmetry which allows spontaneous polarization.

The general consensus about the origin of ferroelectricity in $\mathrm{R}_{2} \mathrm{Mn}_{4} \mathrm{O}_{10}$-compounds is that charge-ordering by magnetostriction is favored over Dzyaloshinskii-Moriya interaction for several reasons. Anisotropic superexchange is expected to be rather weak due to the nearly collinear spin alignment. Many common characteristics are missing that can be found in $\mathrm{MnWO}_{4}$ or $\mathrm{CuO}$, for example a secondary easy-axis, two second-order phase transitions at close temperatures and the switching of the spontaneous polarization by an external magnetic field [102]. Even the occurrence of ferroelectricity in the commensurate phase instead of the incommensurate one, as in $\mathrm{MnWO}_{4}$ and CuO, suggests a different mechanism.

Chapon proposed a model to explain ferroelectricty in $\mathrm{Tb}_{2} \mathrm{Mn}_{4} \mathrm{O}_{10}$ based on charge-ordering due to magnetostriction [21] where the magnetically frustrated spin chain in $\boldsymbol{b}$-direction (blue dashed line in Figure 2-18 (left)) plays an important role. As explained in chapter 2.3.3.2, this chain consists of $\mathrm{Mn}^{3+}-\mathrm{Mn}^{4+}$-pairs coupled by the superexchange interaction $J_{3}$ where the spins of successive pairs are alternatingly parallel and antiparallel oriented. This creates a sequence of "wrong" and "right" pairs whose superexchange contributions to the total free energy in the centrosymmetric structure have the same absolute value but opposite sign and therefore add to zero. A distortion of bond length or angles that could break this magnetic degeneracy by increasing the superexchange of "right" pairs $\left(J_{3,1}\right)$ and decreasing of "wrong" pairs $\left(J_{3,2}\right)$ could lower the free energy while breaking spatial inversion symmetry. Chapon suggests a displacement of $\mathrm{Mn}^{3+}$-ions along the apical axis of the coordination pyramid as depicted in Figure 2-20. Although the electric 
dipoles created by this distortion are pointing mainly along the $\boldsymbol{a}$-axis with only a minor $\boldsymbol{b}$-component, the resulting net polarization can only be observed in $\boldsymbol{b}$-direction because the a-components differ in sign and cancel out. Chapon calls this effect "canted antiferroelectricity" in analogy to the "canted antiferromagnetism" described by Dzyaloshinskii (see chapter 2.2.1) [33].

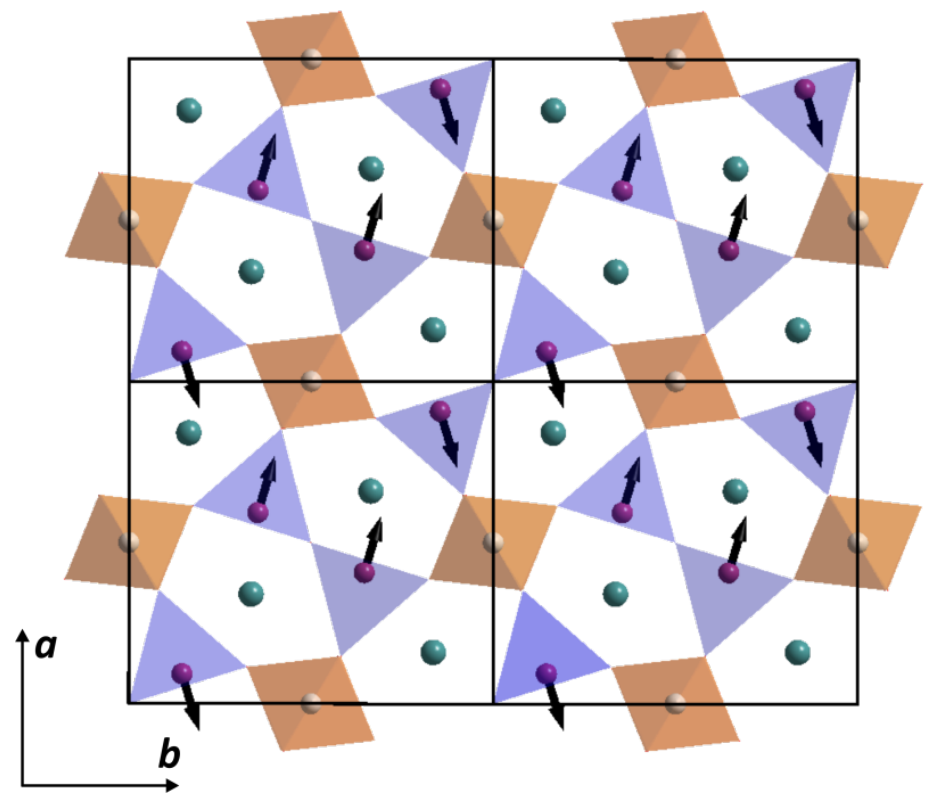

Figure 2-20: Crystal structure of BMO viewing along the c-axis. Black arrows indicate the displacement of $\mathrm{Mn}^{3+}$-ions that cause ferroelectricity. This figure is inspired by [21].

This theory is in accordance with two observations mentioned in chapter 2.3.3.2. The strong contraction of the lattice in a-direction [105] could be a direct result of the displacement of $\mathrm{Mn}^{3+}$-ions in this direction. In addition, the broadening of Bragg-reflections with finite $a$-component below $T_{\mathrm{N}}$ indicates that there is a certain structural modulation along the $\boldsymbol{a}$-axis induced by magnetic ordering. Furthermore, the temperature-dependence of the coordination geometry was recently investigated by Mößbauer spectroscopy [107]. Although the $\mathrm{Mn}^{3+}$-pyramids are closer to their ideal coordination than the $\mathrm{Mn}^{4+}$-octahedra, the pyramids are becoming increasingly distorted upon entering the antiferromagnetic phase while the octahedra remain unaffected. It is therefore safe to assume that the structural modulation in $\boldsymbol{a}$-direction is induced by magnetostriction and tied to a deformation of the coordination geometry around $\mathrm{Mn}^{3+}$. This is exactly what Chapon's model suggests. 


\section{Methods}

All experimental methods that were employed in this thesis are described in the following sections. The first subchapter focuses on different scattering techniques while the determination of the dielectric permittivity and of elastic constants is explained in the second and third subchapter, respectively.

\subsection{Scattering techniques}

There is a wide range of different methods to investigate matter that all base on the same principle: scattering of electromagnetic or particle waves. The properties of the respective waves determine the possible applications and different advantages of each method. All scattering techniques that were employed in this thesis will be explained in the following sections. The first and second subchapters focus on elastic scattering with X-ray and $\gamma$-radiation. The third and fourth subchapters describe inelastic scattering methods that involve visible electromagnetic radiation and neutrons, respectively. A special method to investigate magnetically ordered materials by a polarized neutron beam is finally explained in the last subchapter.

\subsubsection{X-ray powder diffraction}

X-ray powder diffraction (XRPD) is a widely used method to investigate the purity of polycrystalline samples as well as to determine structural properties like lattice parameters. This is possible because the wavelength of X-radiation is in the same order of magnitude like the crystallographic unit cell of common materials. The primary applications in this thesis are monitoring the reaction progress of solid state synthesis and the structural characterization of the final product.

The diffractometer Debyeflex 3003 by Rich. Seiffert \& Co is used in combination with the position sensitive detector PSD-50M by M. Braun (see Figure 3-1). The X-ray source is a copper anode tube by XRD Eigenmann $\mathrm{GmbH}$ and $\mathrm{Cu}-\mathrm{K}_{\alpha}$ radiation with the wavelength $\lambda=1.5406 \AA$ is selected for diffraction. The scattering angles of low-indexed Bragg reflections of many common materials are in the range of a few dozen ${ }^{\circ}$.

The scattering geometry is schematically illustrated in Figure 3-2. The X-ray tube is fixed to the goniometer and does not move during a scan. The sample holder is a tantalum sheet with a small pit in the center which is filled with the powder sample. The holder is attached to the goniometer in such a way that the sample is in the center of the $\theta$-rotation axis. The detector, on the other hand, is mounted to the $2 \theta$-axis of the goniometer. 


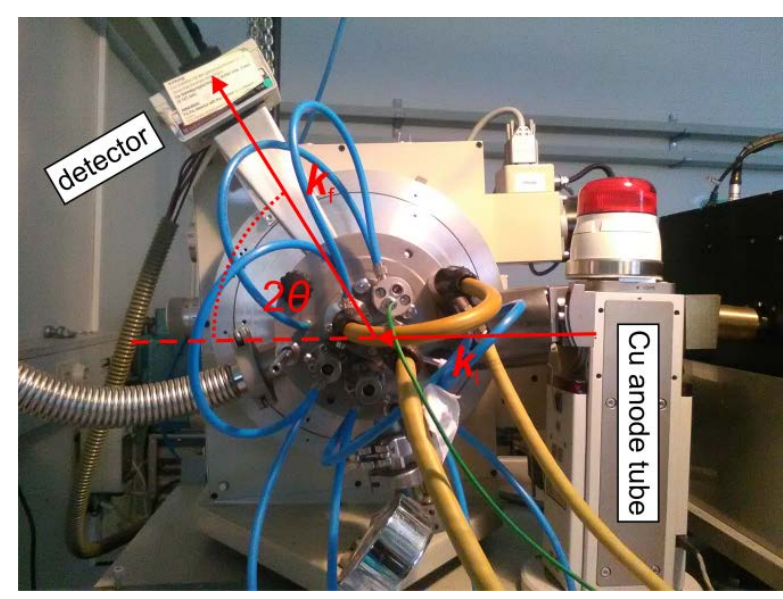

Figure 3-1: Photograph of the X-ray powder diffractometer. Red arrows represent the beam pathway for one arbitrary scattering angle. $\boldsymbol{k}_{\mathrm{i}}$ and $\boldsymbol{k}_{\mathrm{f}}$ are the wavevectors of the initial and scattered beam.

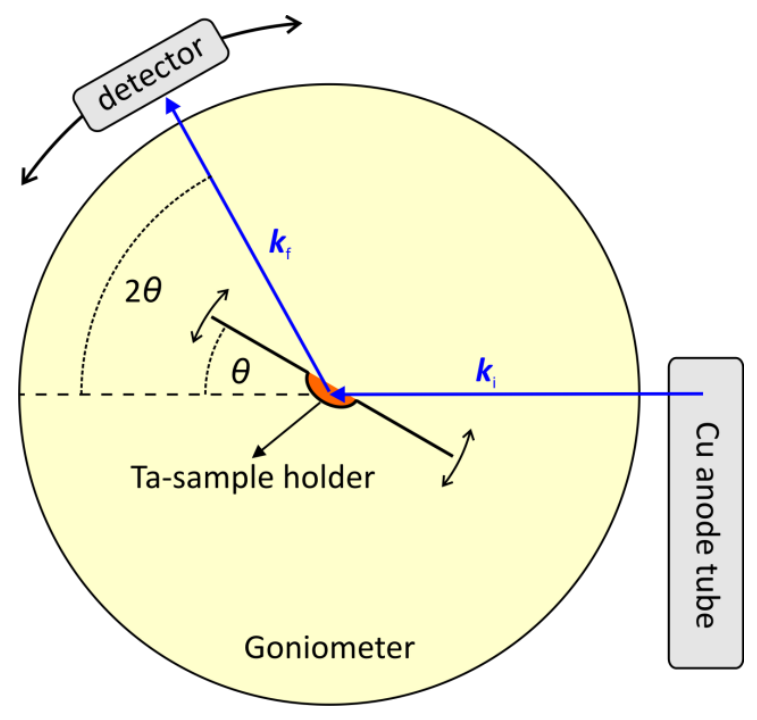

Figure 3-2: Schematic representation of the X-ray powder diffractometer. The orange color in the pit of the sample holder represents the powder sample. $\boldsymbol{k}_{\mathrm{i}}$ and $\boldsymbol{k}_{\mathrm{f}}$ are the wavevectors of the initial and scattered beam.

\subsection{2 $\gamma$-ray diffraction}

Due to its higher energy, $\gamma$-radiation has the important advantage over $\mathrm{X}$-ray that it can penetrate bigger crystals, which are needed for neutron scattering. Intrinsic properties of the bulk can be investigated by $\gamma$-ray diffraction, while $X$-ray diffraction only yields information about the surface of these samples.

The scattering geometry of the $\gamma$-ray diffractometer is schematically depicted in Figure 3-3. The radiation source is ${ }^{192} \mathrm{Ir}$, which emits $\gamma$-radiation with a wavelength of $\lambda=0.0392 \AA$. Due to this small wavelength, common scattering angles are in the range of only $1-2^{\circ}$. This leads to a rather high relative error of $2 \theta$ and therefore a low resolution in reciprocal space parallel to the scattering vector $Q$. The long distances, on the other hand, from source to sample and from sample to detector together with narrow slits before and after the sample provide an excellent collimation of the beam. In combination with the high energy resolution of the radiation due to nuclear decay, the accuracy of the sample rotation axis $\omega$ is in the order of $0.01^{\circ}$ and this method has therefore a high resolution in

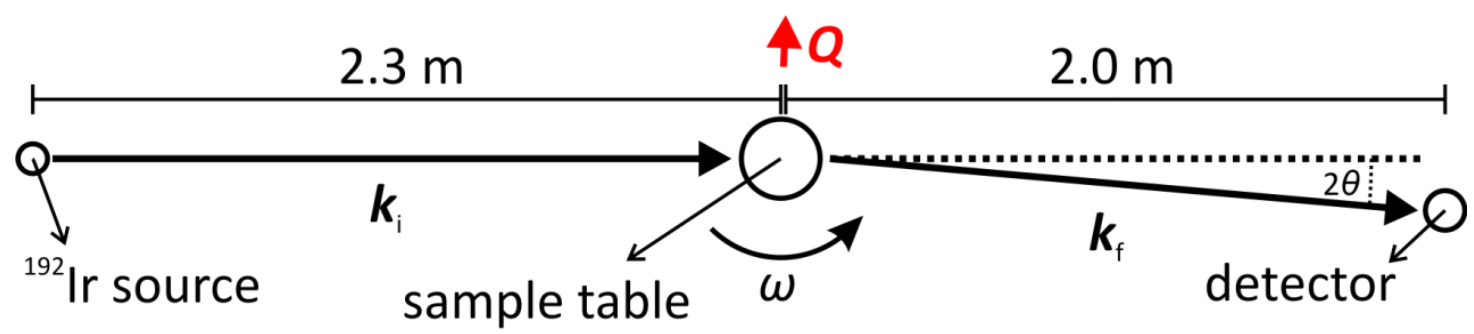

Figure 3-3: Schematic representation of the $\gamma$-ray diffractometer, viewing from above the instrument. $\boldsymbol{k}_{\mathrm{i}}$ and $\boldsymbol{k}_{\mathrm{f}}$ are the wavevectors of the initial and scattered beam. 
reciprocal space perpendicular to $\boldsymbol{Q}$. Consequently, a common application is the precise determination of the sample's mosaicity and the identification of twin crystals and individual subgrains.

\subsubsection{Raman spectroscopy}

Raman spectroscopy is an inelastic scattering technique that involves visible electromagnetic radiation with a wavelength of a few hundred nanometers, which is too high for diffraction of common materials. This technique is rather used for the excitation of rotational and vibrational states of molecules or, as employed in this thesis, for the determination of lattice vibrations in solids. Only phonons very close to the Brillouin-zone center can be measured due to the high wavelength.

The spectrometer $T 64000$ by Horiba Jobin Yvon with three grating monochromator was used in subtractive mode for the measurement of all spectra. The light source was a solid state laser by UniKLasers with a wavelength of $\lambda=639.7 \mathrm{~nm}$.

\subsubsection{Inelastic neutron scattering}

The thermal three-axes spectrometers PUMA@FRMII [112] and IN8@ILL were used for the inelastic neutron scattering experiments of this thesis. The wavelength of thermal neutrons (e.g. $\lambda=2.360 \AA$ ) is in the range of $\mathrm{X}$-radiation while their energy is similar to lattice vibrations. This method therefore allows diffraction experiments as well as the measurement of phonons and magnons in the whole Brillouin-zone of common materials.

Figure 3-4 is the schematic representation of a three-axes spectrometer. The beam that leaves the reactor chamber consists of neutrons with a broad distribution of different wavelengths. The first axis is the monochromator, which is an array of single crystals that select neutrons with a narrow wavelength distribution from the polychromatic beam by Bragg-reflection. The rotation angle of the monochromator therefore determines the kinetic energy of the reflected neutrons. The monochromatic beam then reaches the sample where the actual scattering process takes place. The sample table is the second rotation axis and the angle between the initial beam and the analyzer determines the scattering angle $2 \theta$. Slits before and after the sample table can be adjusted to the sample size in order to reduce the detected noise. The scattered, polychromatic beam reaches the analyzer, which is the third axis and selects one wavelength by Bragg-scattering, similar to the monochromator. Neutrons with the right kinetic energy are reflected towards the ${ }^{3} \mathrm{He}$-detector where they are finally counted. Four Soller-collimators can be placed between the neutron source, 
the monochromator, the sample, the analyzer and the detector. They restrict the beam divergence and therefore increase the spectrometer resolution.

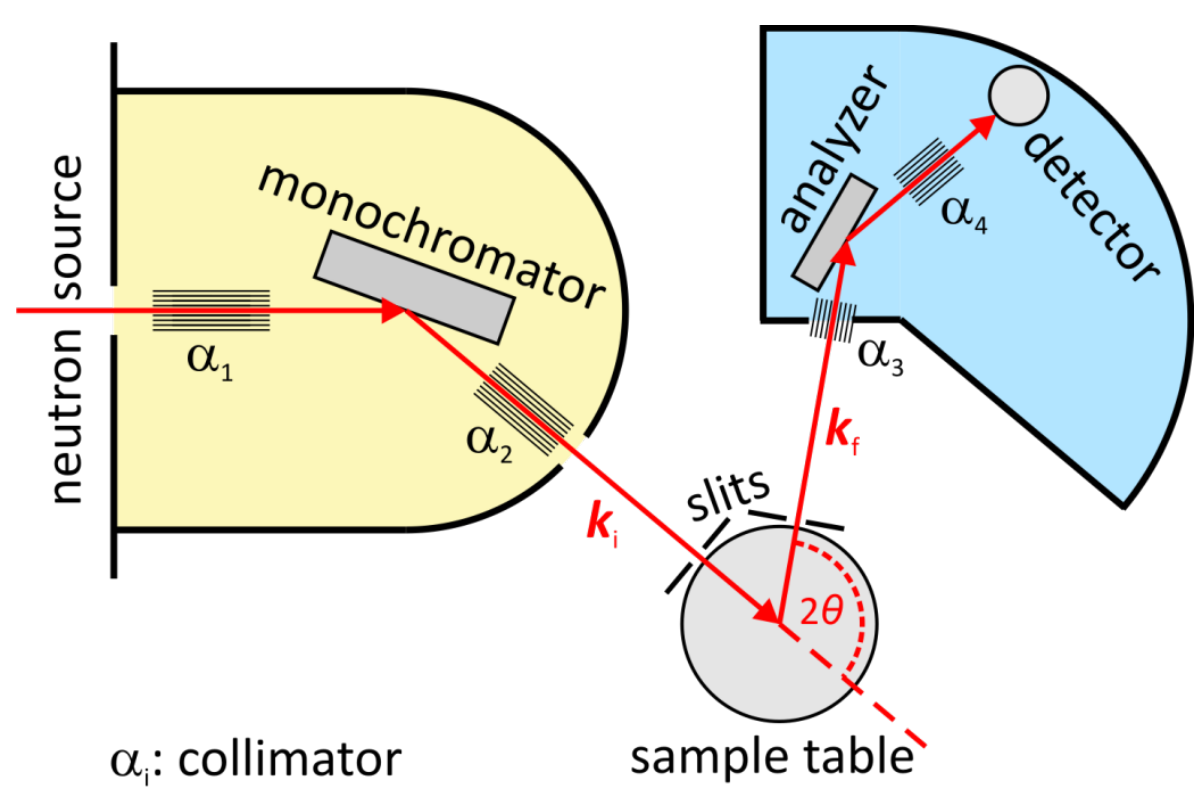

Figure 3-4: Schematic representation of a three-axes spectrometer by the example of PUMA. All four collimators are labeled and enumerated by $\alpha_{i} \cdot \boldsymbol{k}_{\mathrm{i}}$ and $\boldsymbol{k}_{\mathrm{f}}$ are the wave vectors of the initial and scattered neutron beam.

\subsubsection{Polarized neutron scattering}

Polarized neutron scattering is a method to gain information about the magnetic structure and spin dynamics of a material that would not be accessible with conventional neutron scattering. In a perfectly polarized neutron beam, all neutron spins are pointing in the same direction. The orientation of its magnetic moment determines how the neutron will interact with the magnetic moments of a sample. The neutron can be before as well as after the scattering process in 6 different spin states $(x,-x, y,-y, z$ or $-z)$, leading to 36 possible combinations which all contribute to the total scattered intensity. The intensity of each combination results from a specific component of the sample's magnetization. Hence, if it is possible to detect the according intensities separately, detailed information about the magnetic structure are accessible.

In order to achieve this, the polarization state of the neutron spin before and after the sample has to be selected. There are different devices that fulfill this purpose, for example Heusler-crystals, whose Bragg-scattering is polarized, ${ }^{3} \mathrm{He}$-filters, which absorb all polarization states but one due to the interaction with polarized ${ }^{3} \mathrm{He}$-nuclei, or neutron mirrors consisting of magnetic multi-layers, where the angle of total reflection depends on the spin state of the neutron. If the device is placed in front of the sample, it is called "polarizer" while an "analyzer" may be the same device behind the sample. 
In the following subchapter, the selection rules of polarized neutron scattering are explained. These rules describe which specific component of the magnetization is connected to which combination of spin states. The second subchapter is dedicated to the polarization analysis setup of PUMA which was used in this thesis.

\subsubsection{Selection rules}

The interaction of a neutron spin with the magnetic field induced by an electron can be divided in two contributions: orbit- and spin-interaction. Only the latter is important for the determination of the magnetic structure. The contribution of the spin-scattering to the structure factor can be described as:

$$
\left\langle\boldsymbol{k}_{\mathrm{f}} \boldsymbol{\sigma}_{\mathrm{f}} \lambda_{\mathrm{f}}\left|V_{\mathrm{spin}}(r)\right| \boldsymbol{k}_{\mathrm{i}} \boldsymbol{\sigma}_{\mathrm{i}} \lambda_{\mathrm{i}}\right\rangle=4 \pi\left(\gamma \mu_{k}\right) \delta\left(\hbar \omega+E_{\mathrm{i}}-E_{\mathrm{f}}\right)\left\langle\boldsymbol{\sigma}_{\mathrm{f}} \lambda_{\mathrm{f}}\left|\sum_{k} \exp \left(i Q \boldsymbol{R}_{k}\right) \hat{\boldsymbol{\sigma}} \boldsymbol{s}_{k}^{\perp}\right| \boldsymbol{\sigma}_{\mathrm{i}} \lambda_{\mathrm{i}}\right\rangle
$$

where $\boldsymbol{k}_{\mathrm{i} / \mathrm{f}}, \sigma_{\mathrm{i} / \mathrm{f}}, E_{\mathrm{i} / \mathrm{f}}$ and $\lambda_{\mathrm{i} / \mathrm{f}}$ are the wave vector, spin state, energy and wavelength of the initial or scattered neutron, respectively. $\gamma$ is the gyromagnetic ratio and $\mu_{\mathrm{k}}$ is the nuclear magneton. $\boldsymbol{Q}$ is the scattering vector and $\sigma$ is the Pauli spin operator, while $\boldsymbol{R}_{\mathrm{k}}$ is the position of the $\mathrm{k}^{\text {th }}$-electron and $\boldsymbol{s}_{\mathrm{k}} \perp$ is its spin component orthogonal to $\boldsymbol{Q}$. The latter can be described in terms of the magnetic structure of the substance:

$$
\left\langle\boldsymbol{\sigma}_{\mathrm{f}} \lambda_{\mathrm{f}}\left|\sum_{k} \exp \left(i \boldsymbol{Q} \boldsymbol{R}_{k}\right) \hat{\boldsymbol{\sigma}} \boldsymbol{s}_{k}^{\perp}\right| \boldsymbol{\sigma}_{\mathrm{i}} \lambda_{\mathrm{i}}\right\rangle=\left\langle\boldsymbol{\sigma}_{\mathrm{f}} \lambda_{\mathrm{f}}\left|\hat{\boldsymbol{\sigma}}_{x} m_{x}+\hat{\boldsymbol{\sigma}}_{y} m_{y}+\hat{\boldsymbol{\sigma}}_{z} m_{z}\right| \boldsymbol{\sigma}_{\mathrm{i}} \lambda_{\mathrm{i}}\right\rangle
$$

$m_{x}, m_{y}$ and $m_{z}$ are the components of the Fourier-transformed spins. The Pauli spin operators and their respective eigenvectors are:

$$
\begin{array}{lll}
\hat{\boldsymbol{\sigma}}_{x}=\left(\begin{array}{ll}
0 & 1 \\
1 & 0
\end{array}\right) & \boldsymbol{\sigma}_{x}^{+}=\frac{1}{\sqrt{2}}\left(\begin{array}{l}
1 \\
1
\end{array}\right) & \boldsymbol{\sigma}_{x}^{-}=\frac{1}{\sqrt{2}}\left(\begin{array}{c}
1 \\
-1
\end{array}\right) \\
\hat{\boldsymbol{\sigma}}_{y}=\left(\begin{array}{cc}
0 & -i \\
i & 0
\end{array}\right) & \boldsymbol{\sigma}_{y}^{+}=\frac{1}{\sqrt{2}}\left(\begin{array}{l}
1 \\
i
\end{array}\right) & \boldsymbol{\sigma}_{y}^{-}=\frac{1}{\sqrt{2}}\left(\begin{array}{c}
1 \\
-i
\end{array}\right) \\
\hat{\boldsymbol{\sigma}}_{z}=\left(\begin{array}{ll}
1 & 0 \\
0 & 1
\end{array}\right) & \boldsymbol{\sigma}_{z}^{+}=\left(\begin{array}{l}
1 \\
0
\end{array}\right) & \boldsymbol{\sigma}_{z}^{-}=\left(\begin{array}{l}
0 \\
1
\end{array}\right)
\end{array}
$$

The initial as well as the scattered neutron can be in one of these 6 spin states. Two of the 36 possible combinations shall be examined at this point. If the neutron spin is initially oriented in positive $\mathbf{z}$-direction and keeps this orientation during the scattering process, the following expression can be derived, omitting the wavelength: 


$$
\begin{aligned}
& \left\langle\boldsymbol{\sigma}_{z}^{+}\left|\hat{\boldsymbol{\sigma}}_{x} m_{x}+\hat{\boldsymbol{\sigma}}_{y} m_{y}+\hat{\boldsymbol{\sigma}}_{z} m_{z}\right| \boldsymbol{\sigma}_{z}^{+}\right\rangle=\left\langle\boldsymbol{\sigma}_{z}^{+}\left|\hat{\boldsymbol{\sigma}}_{x} m_{x}\right| \boldsymbol{\sigma}_{z}^{+}\right\rangle+\left\langle\boldsymbol{\sigma}_{z}^{+}\left|\hat{\boldsymbol{\sigma}}_{y} m_{y}\right| \boldsymbol{\sigma}_{z}^{+}\right\rangle+\left\langle\boldsymbol{\sigma}_{z}^{+}\left|\hat{\boldsymbol{\sigma}}_{z} m_{z}\right| \boldsymbol{\sigma}_{z}^{+}\right\rangle \\
& =\left(\begin{array}{l}
1 \\
0
\end{array}\right)\left(\begin{array}{ll}
0 & 1 \\
1 & 0
\end{array}\right)\left(\begin{array}{l}
1 \\
0
\end{array}\right) m_{x}+\left(\begin{array}{c}
1 \\
0
\end{array}\right)\left(\begin{array}{cc}
0 & -i \\
i & 0
\end{array}\right)\left(\begin{array}{l}
1 \\
0
\end{array}\right) m_{y}+\left(\begin{array}{l}
1 \\
0
\end{array}\right)\left(\begin{array}{ll}
1 & 0 \\
0 & 1
\end{array}\right)\left(\begin{array}{l}
1 \\
0
\end{array}\right) m_{z}=m_{z}
\end{aligned}
$$

If the orientation of the neutron spin is, however, flipped, the result is different:

$$
\begin{aligned}
& \left\langle\boldsymbol{\sigma}_{z}^{+}\left|\hat{\boldsymbol{\sigma}}_{x} m_{x}\right| \boldsymbol{\sigma}_{z}^{-}\right\rangle+\left\langle\boldsymbol{\sigma}_{z}^{+}\left|\hat{\boldsymbol{\sigma}}_{y} m_{y}\right| \boldsymbol{\sigma}_{z}^{-}\right\rangle+\left\langle\boldsymbol{\sigma}_{z}^{+}\left|\hat{\boldsymbol{\sigma}}_{z} m_{z}\right| \boldsymbol{\sigma}_{z}^{-}\right\rangle \\
& =\left(\begin{array}{l}
1 \\
0
\end{array}\right)\left(\begin{array}{ll}
0 & 1 \\
1 & 0
\end{array}\right)\left(\begin{array}{l}
0 \\
1
\end{array}\right) m_{x}+\left(\begin{array}{c}
1 \\
0
\end{array}\right)\left(\begin{array}{cc}
0 & -i \\
i & 0
\end{array}\right)\left(\begin{array}{l}
0 \\
1
\end{array}\right) m_{y}+\left(\begin{array}{l}
1 \\
0
\end{array}\right)\left(\begin{array}{ll}
1 & 0 \\
0 & 1
\end{array}\right)\left(\begin{array}{l}
0 \\
1
\end{array}\right) m_{z}=m_{x}-i \cdot m_{y}
\end{aligned}
$$

A coordinate system is chosen where $\boldsymbol{x}$ is parallel to $\boldsymbol{Q}, \boldsymbol{y}$ is perpendicular to $\boldsymbol{Q}$ within the scattering plane and $\boldsymbol{z}$ is perpendicular to the scattering plane. All spin components along $\boldsymbol{x}$ are not orthogonal to $Q$ and therefore $m_{\mathrm{x}}=0$. The scattered intensity is proportional to the square of the structure factor. In the previous example of the spin flipped and non-spin flipped neutron which was initially polarized along $z$, the resulting intensities are $I_{\mathrm{SF}} \propto\left|m_{\mathrm{y}}\right|^{2}$ and $I_{\mathrm{NSF}} \propto\left|m_{\mathrm{z}}\right|^{2}$, respectively. An experimental setup to determine these two intensities was developed already decades ago [113]. For collinear spins, these two contributions contain all information about the magnetic structures of the substance. If the spins are, however, not collinear, $m_{\mathrm{y}}$ and $m_{\mathrm{z}}$ may be complex quantities and other combinations of spin states have to be considered. The results for all 36 possible combinations are listed in Table 3-1.

Table 3-1: Scattered intensity depending on the polarization of the initial neutron (columns) and the scattered neutron (rows).

\begin{tabular}{|c|c|c|c|c|c|c|}
\hline & $x$ & $-x$ & $y$ & $-y$ & $z$ & $-z$ \\
\hline$x$ & 0 & $2 G$ & $G$ & $G$ & $G$ & $G$ \\
\hline$-\boldsymbol{x}$ & $2 F$ & 0 & $F$ & $F$ & $F$ & $F$ \\
\hline $\boldsymbol{y}$ & $F$ & $G$ & $A$ & $B$ & $C$ & $D$ \\
\hline$-\boldsymbol{y}$ & $F$ & $G$ & $B$ & $A$ & $D$ & $C$ \\
\hline $\boldsymbol{z}$ & $F$ & $G$ & $C$ & $D$ & $B$ & $A$ \\
\hline$-\boldsymbol{z}$ & $F$ & $G$ & $D$ & $C$ & $A$ & $B$ \\
\hline
\end{tabular}

The abbreviations are:

$$
\begin{aligned}
& A=\left|m_{y}\right|^{2} \quad B=\left|m_{z}\right|^{2} \\
& C=1 / 2\left(\left|m_{y}\right|^{2}+\left|m_{z}\right|^{2}+m_{y}^{*} m_{z}+m_{y} m_{z}^{*}\right) \\
& D=1 / 2\left(\left|m_{y}\right|^{2}+\left|m_{z}\right|^{2}-m_{y}^{*} m_{z}-m_{y} m_{z}^{*}\right) \\
& F=1 / 2\left(\left|m_{y}\right|^{2}+\left|m_{z}\right|^{2}+i\left(m_{y}^{*} m_{z}-m_{y} m_{z}^{*}\right)\right) \\
& G=1 / 2\left(\left|m_{y}\right|^{2}+\left|m_{z}\right|^{2}-i\left(m_{y}^{*} m_{z}-m_{y} m_{z}^{*}\right)\right)
\end{aligned}
$$

\subsubsection{Polarization analysis setup of PUMA}

The polarization analysis setup is an optional upgrade of PUMA and is used in this thesis to determine the eigenvector of magnetic excitations in the multiferroic phase of $\mathrm{CuO}$ (chapter 5 ). The basic functionality and some key features will be explained at this point, while a further detailed explanation is given in [114]. For the initial polarization, a ${ }^{3} \mathrm{He}$-filter is used which polarizes the 
neutron beam in z-direction. The role of the spin analyzer, on the other hand, is fulfilled by two deflectors which allow the distinction between $\mathbf{z}$ - and $\mathbf{- z}$-polarization. Two different spin contributions can therefore be detected with this setup: spin-flip (SF) and non-spin-flip (NSF) which correspond to $I_{\mathrm{SF}} \propto\left|m_{\mathrm{y}}\right|^{2}$ and $I_{\mathrm{NSF}} \propto\left|m_{\mathrm{z}}\right|^{2}$, respectively.

All relevant components of the polarization analysis setup are shown in Figure 3-5, Figure 3-6 and Figure 3-7. The monochromator, primary collimator, secondary collimator and virtual source are, however, not depicted because they are the same as in the conventional setup. The neutron beam that is reflected by the graphite-monochromator is completely depolarized as represented by an alternatingly green and red dashed line in Figure 3-5. Red color is associated to neutrons with an upward spin perpendicular to the scattering plane and neutrons with downward spin are colored green. The initial beam also contains neutrons with any other spin orientation.

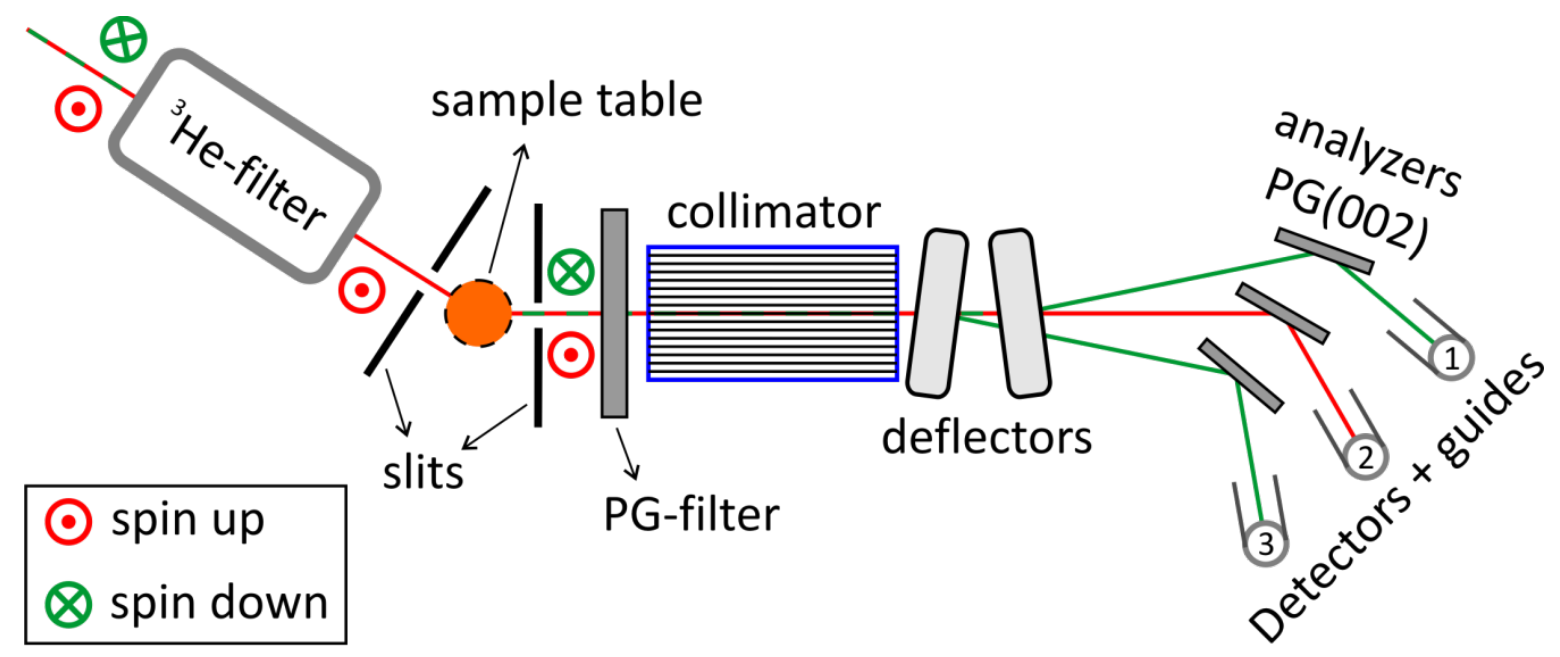

Figure 3-5: Schematic representation of the polarization analysis setup of PUMA@FRMII. The image plane is equal to the scattering plane and it is viewed from above the instrument. The monochromator, primary collimator, secondary collimator and virtual source are not depicted because they are the same as in the conventional setup. See text for details about the individual components.

Next, the neutron beam enters the ${ }^{3} \mathrm{He}$-cell which is filled with ${ }^{3} \mathrm{He}$-gas whose nuclear spins are aligned perpendicular to the scattering plane. This is possible because the cell is equipped with a permanent magnet that creates a magnetic field in the same direction and thereby stabilizes the ${ }^{3} \mathrm{He}$-polarization. Furthermore, all neutron spins will align either parallel or antiparallel to this field. The neutron absorbance of polarized ${ }^{3} \mathrm{He}$-gas strongly depends on the orientation of the neutron spin. In this particular case, most of the down-spin neutrons are absorbed. Hence, the neutron beam that leaves the ${ }^{3} \mathrm{He}$-filter and reaches the sample predominantly contains spin up neutrons. This comes with the cost of losing approximately $70 \%$ of the intensity. The total absorbance and the polarization $P_{0}$ of the transmitted beam depend on the pressure and polarization of the ${ }^{3} \mathrm{He}$-gas. The latter decays over time and the results must therefore be correct accordingly. 
Slits before and after the sample table can be adjusted to the sample size. They are covered with boron rubber in order to reduce the background noise caused by neutrons that are not scattered by the sample and could find their way to the detector. The sample table of the conventional setup is used which can be equipped with further devices like for example a cryostat to cool the sample below room temperature. The neutron beam that is scattered by the sample passes the PG-filter and the collimator before it reaches both deflectors. Like the ${ }^{3} \mathrm{He}$-filter, the collimator as well as the deflectors are equipped with permanent magnets that create a guide field perpendicular to the scattering plane in order to prevent the neutron beam from depolarizing. For the same purpose, two quasi Helmholtz coils are mounted above and below the sample (see Figure 3-6) to achieve a homogenous field even inside the cryostat. After entering the ${ }^{3} \mathrm{He}$-filter, the neutron beam is therefore continuously exposed to guide fields until it exits the deflectors.

The deflectors are stacks of Si-wavers coated with magnetized layers of FeSi that can reflect a neutron beam, but the angle of total reflection depends on the spin orientation. The beam that reaches the deflectors may contain neutrons with both spin states, depending on the scattering characteristics of the sample. The deflectors are rotated in such a way that spin down neutrons are reflected while spin up neutrons can pass. Because of the beam divergence that is higher than the deflector's angular range for reflection, two deflectors are used in order to reflect spin down neutrons with all possible divergence angles. Furthermore, the reflectivity of the deflectors is only $70 \%$ so some of the neutrons that were not reflected by the first deflector might be reflected by the second one.

Behind the deflectors, no guide fields are needed anymore because neutrons with different spin orientation are already separated in three distinct beams. Each of these beams reaches a different PG(002)-analyzer of the PUMA multianalyzer system and all neutrons with the right wavelength are reflected to three seperate detectors (see Figure 3-7 for a picture of the optical path). The two deflected beams of detector 1 and 3 correspond to spin down neutrons (SF-scattering) while the transmitted beam of detector 2 consists mainly of spin up neutrons (NSF-scattering) and some spin down ones that were not reflected by the deflectors. This is, however, only true in first approximation. In fact, there are SF- as well as NSF-contributions in all three detectors due to the imperfectly polarized neutron beam $\left(P_{0}\right)$ leaving the ${ }^{3} \mathrm{He}$-filter. The real SFand NSF-intensities can be calculated according to: 


$$
\begin{aligned}
& I_{\mathrm{SF}}=1 / 2\left[\left(1-\frac{1}{P_{0}}\right) I_{2}+\left(\frac{D_{+}}{P_{0}}-D_{-}\right)\left(I_{1}+I_{3}\right)\right] \\
& I_{\mathrm{NSF}}=1 / 2\left[\left(1+\frac{1}{P_{0}}\right) I_{2}-\left(\frac{D_{+}}{P_{0}}+D_{-}\right)\left(I_{1}+I_{3}\right)\right]
\end{aligned}
$$

where $I_{\mathrm{i}}$ is the count rate of the $\mathrm{i}^{\text {th }}$ detector, $P_{0}$ is the initial polarization of the neutron beam before reaching the sample and $D_{+}$as well as $D_{\text {. }}$ are parameters that are specific to the instrumental setup and can be easily calculated by geometric considerations [114]. These two intensities, $I_{\mathrm{SF}}$ and $I_{\text {NSF, }}$ are the essential quantities determined by the polarization analysis setup of PUMA.

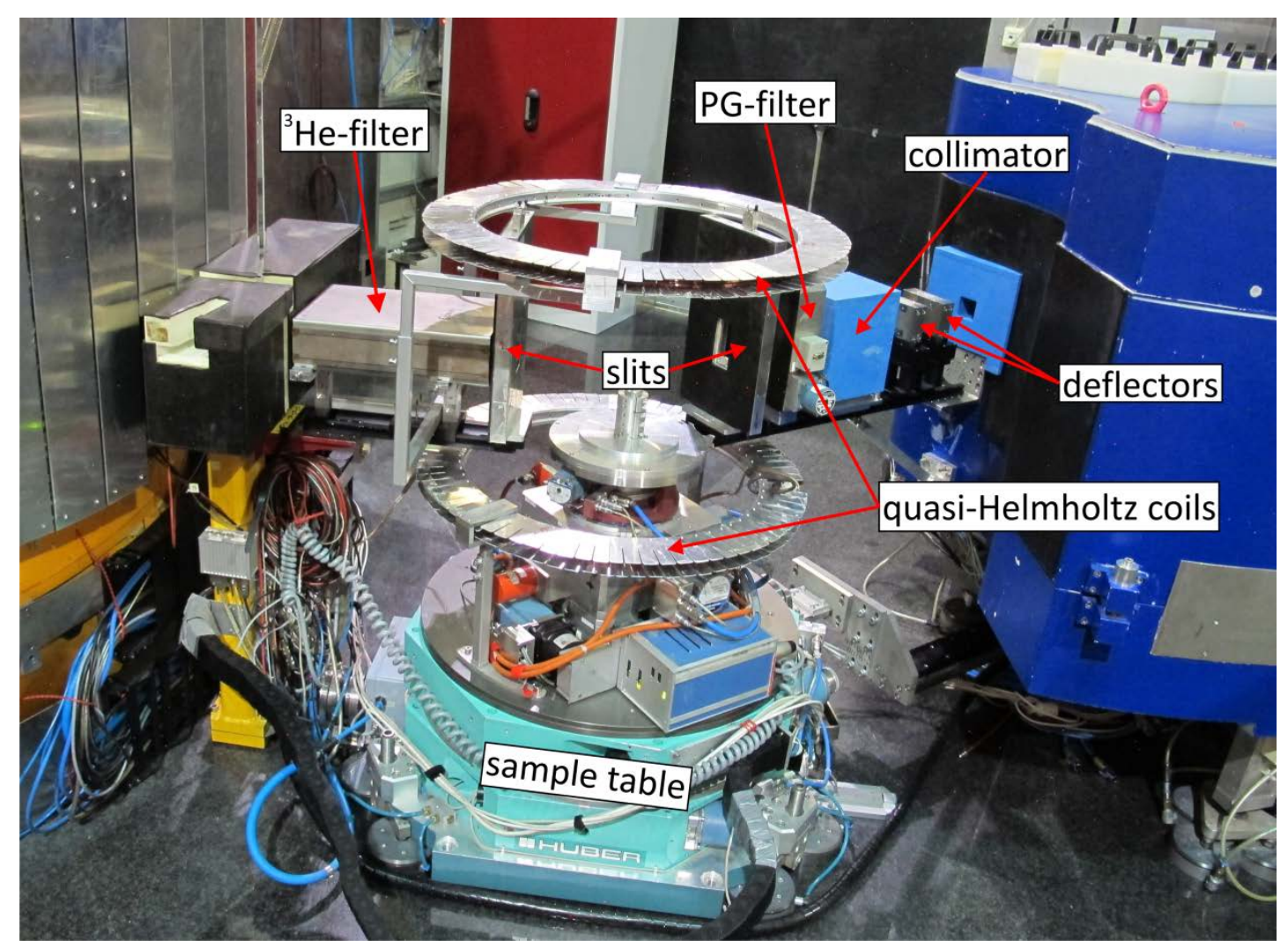

Figure 3-6: Picture of the polarization analysis setup of PUMA@FRMII. See text for details about the individual components. 


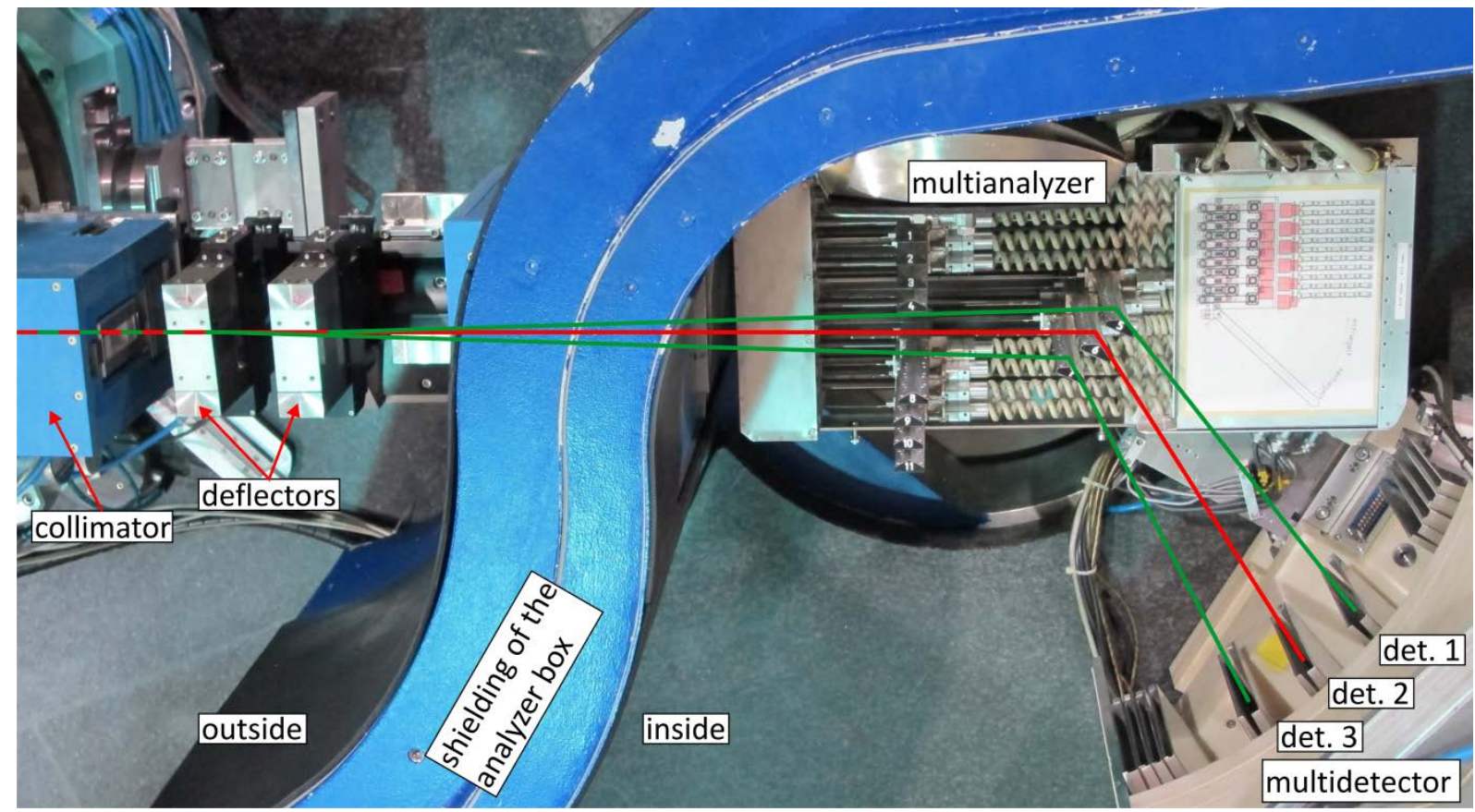

Figure 3-7: Picture of the multianalyzer and multidetecor system of PUMA together with the collimator and deflectors of the polarization analysis setup. The path of the transmitted and the two reflected beams are represented by red and green lines, respectively.

\subsection{Dielectric investigation}

The dielectric susceptibility $\chi$ is a two-dimensional tensor that describes the induced polarization $\boldsymbol{P}$ when an external electric field $\boldsymbol{E}$ is applied. It can also be expressed in terms of the relative dielectric permittivity $\boldsymbol{\varepsilon}_{\mathrm{r}}$ :

$$
\boldsymbol{P}=\boldsymbol{\chi} \varepsilon_{0} \boldsymbol{E}=\left(\boldsymbol{\varepsilon}_{\mathrm{r}}-1\right) \varepsilon_{0} \boldsymbol{E}
$$

$\chi$ is a uniform second order ferroic in Schmid's power series of the free energy by external fields (see equation (2-5) in chapter 2.1.2). The determination of this quantity is essential for the investigation of magnetoelectric multiferroics. If a material is a type-II multiferroic, it is expected to change its electric properties upon entering the magnetically ordered phase. The dielectric permittivity should therefore differ above and below $T_{\mathrm{N}}$ and, even more noticeable, there is a pronounced anomaly of the permittivity at $T_{\mathrm{N}}$. The anomaly affects the component of $\varepsilon_{\mathrm{r}}$ along the direction of the spontaneous electric polarization. This is a common feature of ferroelectric phase transitions and also the reason why impedance spectroscopy is a wide-spread tool in multiferroic research. This technique uses the fact that the capacity of a material $C$ inside a plate capacitor is proportional to its relative permittivity: 


$$
C=\varepsilon_{\mathrm{r}} C_{\mathrm{p}}^{0}=\left(\varepsilon_{\mathrm{r}}^{\prime}+i \varepsilon_{\mathrm{r}}^{\prime \prime}\right) C_{\mathrm{p}}^{0}
$$

$\varepsilon_{\mathrm{r}}$ is the component of the permittivity tensor that corresponds to the direction of the normal vector of the capacitor plate. Please note that $\varepsilon_{\mathrm{r}}$ is in general a complex quantity. $C_{\mathrm{P}}{ }^{0}$ is the vacuum capacity of the plate capacitor without any sample and therefore $\varepsilon_{\mathrm{r}}=1$. The measurement of the capacity can be illustrated by an RC-circuit as depicted in Figure 3-8. The sample is represented by red color and placed in between the plates of a capacitor, which is connected in series with a resistor $R$. An AC voltage $U_{1}$ is applied to the RC circuit while the voltage drop $U_{2}$ at the resistor is simultaneously measured. The ratio of both voltages depends on $C, R$ and on the angular frequency $\omega$ of the applied voltage:

$$
\frac{U_{2}}{U_{1}}=\frac{R}{R+\frac{1}{i \omega C}}=\frac{i \omega R C}{i \omega R C+1}=\frac{(\omega R C)^{2}}{(\omega R C)^{2}+1}+i \frac{\omega R C}{(\omega R C)^{2}+1}=\mathfrak{R}+i \mathfrak{I}
$$

Due to the phase shift between both voltages, this ratio is a complex quantity. The real part $\Re$ and the imaginary part $\mathfrak{J}$ of this voltage ratio are plotted against the logarithmic angular frequency in Figure 3-9. $\mathfrak{I}$ exhibits a maximum at $\omega=(R C)^{-1}$, which will further on be called resonance frequency $\omega^{*} . \Re$, on the other hand, is a step-like function, which is centered around $\omega^{*} . \Re$ as well as $\mathfrak{J}$ are measured during the experiment and allow the calculation of the real and imaginary part of the permittivity $\varepsilon_{r}^{\prime}$ and $\varepsilon_{r}^{\prime \prime}$, respectively.

The ideal RC-circuit is, however, an oversimplification and only has the purpose to demonstrate the fundamental basics of the technique. An overview over the experimental setup with all necessary additional components will be provided in the next subchapter, while the second

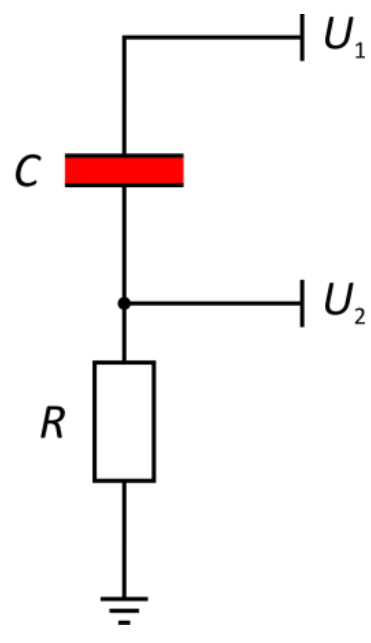

Figure 3-8: Circuit diagram of an RC-circuit. The sample capacitor is marked by red color.

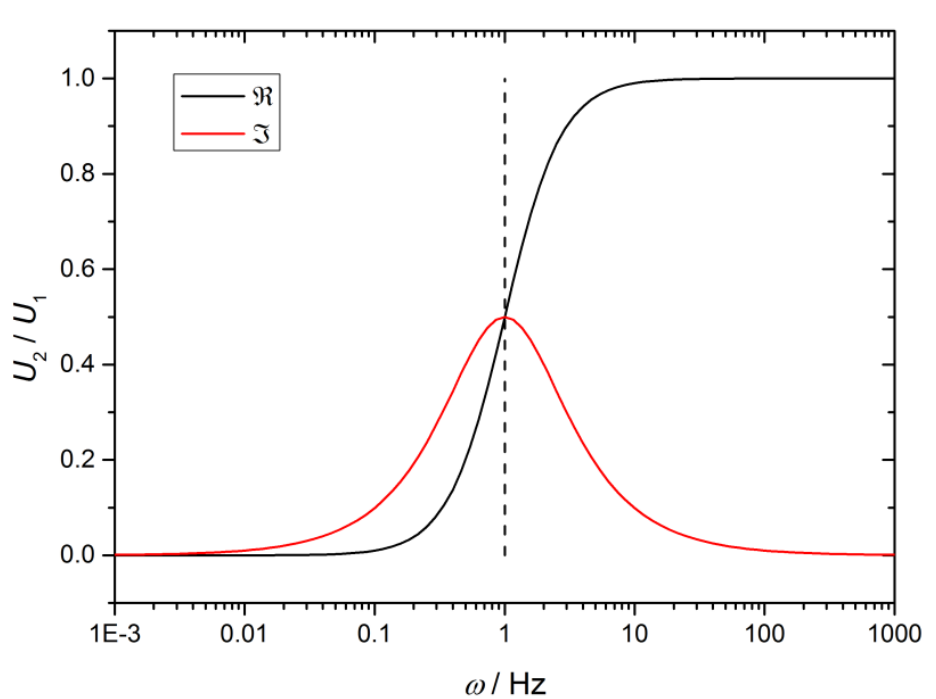

Figure 3-9: Plot of the real and imaginary part of the ratio of $U_{2}$ and $U_{1}$ against the logarithmic angular frequency. The resonance frequency is arbitrarily set to $(R C)^{-1}=1 \mathrm{~Hz}$ for demonstration purposes. 
subchapters will present the complete circuit diagram and a detailed explanation of the data evaluation. The last subchapter will finally focus on the characteristics of Debye relaxations in dielectric materials.

\subsubsection{Experimental setup}

Since the dielectric permittivity of BMO shall be determined at low temperatures around $T_{\mathrm{N}}$, the sample capacitor is built into a helium closed-cycle cryostat by Sumitomo Heavy Industries (SHIRDK-2025 D). The detailed setup is illustrated in Figure 3-10. The copper cold head is cooled down to $4 \mathrm{~K}$ unless the temperature is regulated by a heater, which is directly inserted into the cold head. Furthermore, a Si-diode is screwed to the bottom of the cold head and measures the temperature that is used as control variable by a Lakeshore 330 temperature controller.

An aluminium plate is mounted on top of the cold head and serves as platform for a simple attachment of other devices like the sample holder, which is a cylinder made of aluminium. The lower electrode of the capacitor, which is also made of aluminium, is glued to the sample holder with the two component epoxy resin Uhu plus schnellfest, which has the further purpose to guarantee an electric isolation between electrode and sample holder. The sample is placed between both

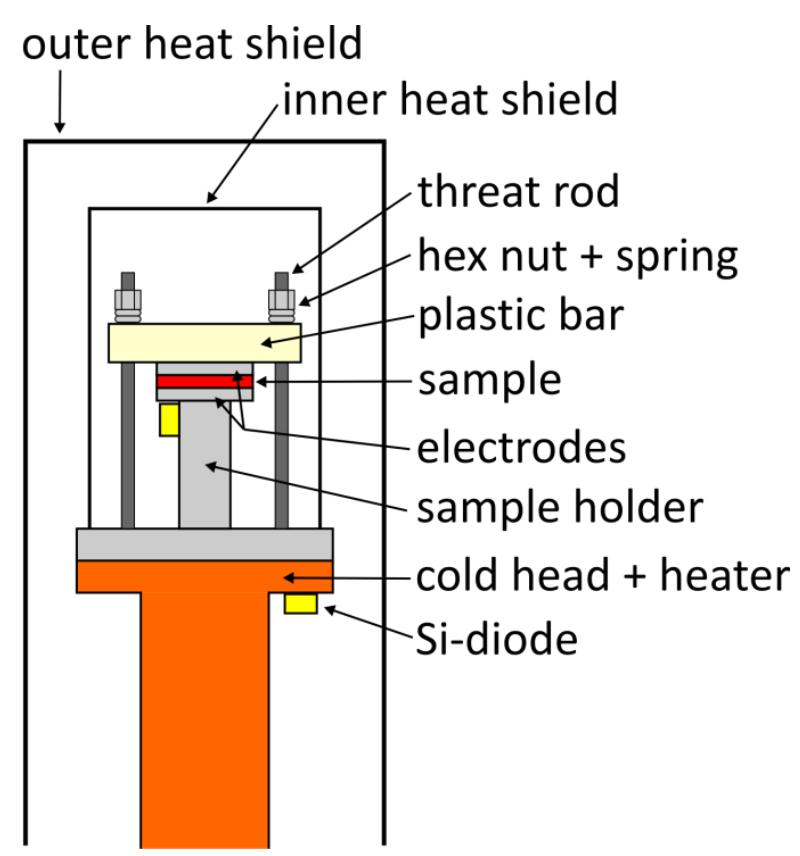

Figure 3-10: Schematic representation of the capacitor inside the cryostat. All relevant components are labeled. A detailed description is given in the text. Wires and the vacuum shield are not depicted for the sake of clarity.

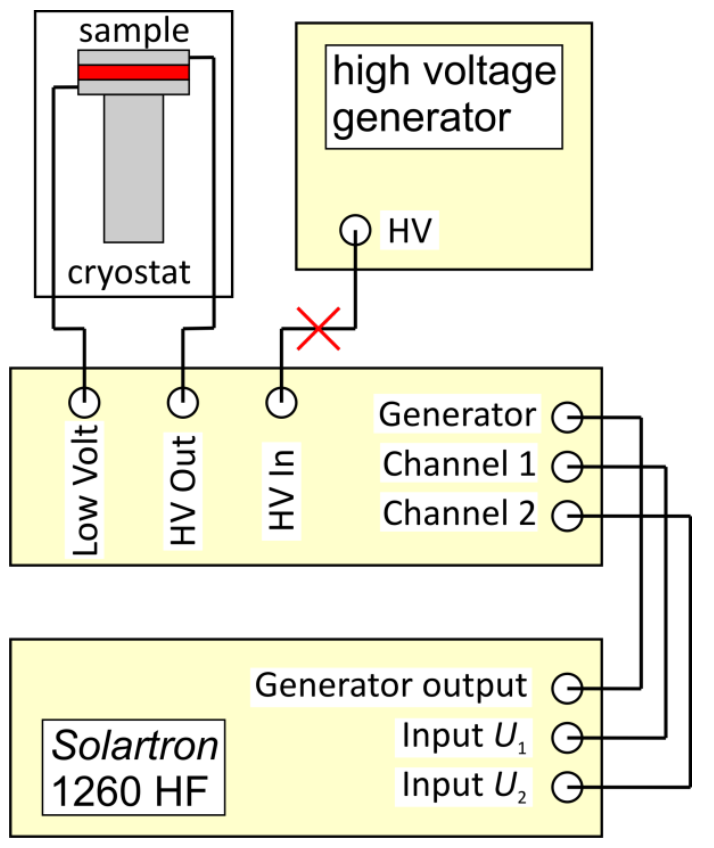

Figure 3-11: Schematic representation of the wiring between different components of the setup. In the bottom is the Impedance spectrometer Solartron $1260 \mathrm{HF}$, which is connected to the high voltage decoupling device that is depicted in the middle. In the top left is a schematic representation of the sample capacitor inside the cryostat, while a high voltage generator is shown in the top right. 
electrodes, but this construction will only be stable if there is constant pressure on the upper electrode from above. In order to achieve this, two threat rods are attached on opposite sides of the sample holder to the platform. A plastic bar with two holes is then slid over the rods and pressed onto the upper electrode with springs and hex nuts. This setup secures a proper contact between sample and electrodes. Another Si-diode is screwed to the sample holder as close to the capacitor as possible to measure the temperature of the sample.

In order to reduce the heat radiation that reaches the sample, an inner heat shield is attached to the platform that is in thermal equilibrium with the cold head. The outer heat shield is equally connected to the first cooling stage of the cryostat. The vacuum shield of the cryostat is not depicted in Figure 3-10 as well as the wires that are connected to the electrodes and Si-diodes.

The rest of the experimental setup outside the cryostat is illustrated in Figure 3-11. On the bottom is the impedance spectrometer Solartron $1260 \mathrm{HF}$ Frequency Response Analyzer. It has a "Generator output" for the applied AC voltage $U_{0}$ and two input channels for the voltages $U_{2}$ and $U_{1}$. This setup was initially constructed to apply a direct high voltage while the permittivity is measured. Therefore, there is an additional device that decouples the high voltage from the rest of the signal and is depicted in the middle of Figure 3-11. Its functionality and circuit diagram will be discussed in detail in the next chapter. It is connected to the output and input channels of the Solartron, but also has three further connections. "Low Volt" is connected to the lower electrode of the sample capacitor while "HV Out" is meant to apply high voltage to the upper electrode. Furthermore, a high voltage generator can be connected to "HV In".

\subsubsection{Circuit diagrams and data evaluation}

The circuit diagram of the experimental setup with high voltage decoupling device is depicted in Figure 3-12. The central part is the sample capacitor $C_{\mathrm{P}}$, which is highlighted by a dashed red rectangle. The AC voltage $U_{0}$ is directly applied to this capacitor. The part of the circuit that connects the sample capacitor to the direct high voltage $\mathrm{HV}$ is not depicted but only indicated by an orange arrow, because no high voltage was applied during the present experiment. In order to separate the high voltage from the measuring channels $U_{1}$ and $U_{2}$, there are high-pass filters, which are marked by blue dashed rectangles. A high-pass filter is an RC-circuit that blocks any signal with a frequency below the resonance frequency of $\omega^{*}=(R C)^{-1}$ (compare to Figure 3-9). The values of the according capacitors and resistors are listed in Table 3-2 and the resulting resonance frequencies are $\omega_{1}{ }^{*}$ $=100 \mathrm{~Hz}$ and $\omega_{2}{ }^{*}=133 \mathrm{~Hz} . R_{0}$ is part of the sample RC-circuit and its resistance can be varied. $C_{0}, C_{1+}$, $C_{2+}$ and $C_{3+}$ are wire capacities which are marked by a green dashed rectangle. 


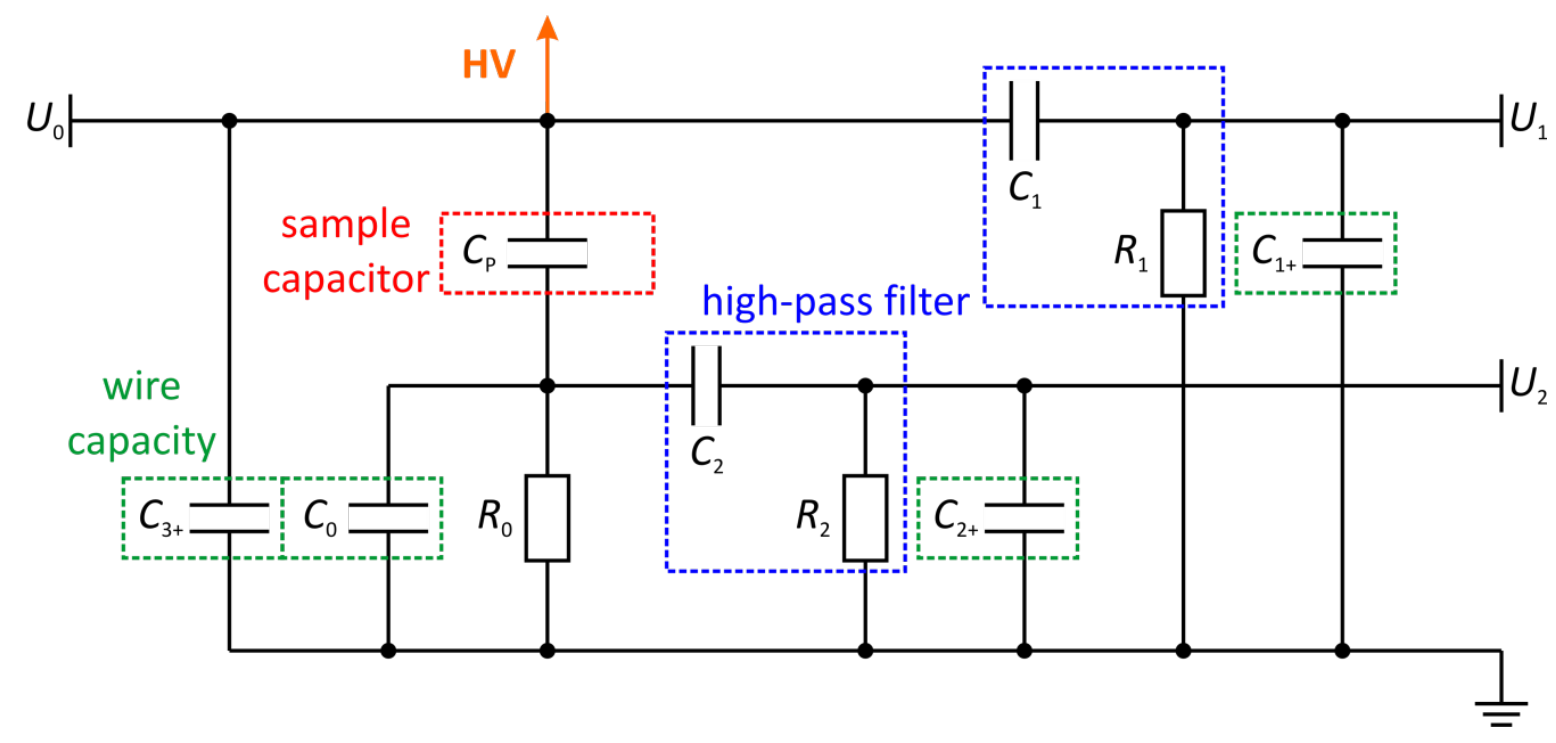

Figure 3-12: Circuit diagram of the setup with high voltage decoupling device. The sample capacitor $\left(C_{\mathrm{P}}\right)$ is highlighted by a red dashed rectangle. The combinations of capacitor $\left(C_{1}\right.$ and $\left.C_{2}\right)$ and resistance $\left(R_{1}\right.$ and $\left.R_{2}\right)$ that are marked by blue dashed rectangles are high-pass filters, which decouple the measuring channels $U_{1}$ and $U_{2}$ from the direct high voltage HV. The part of the circuit that connects the sample capacitor to the high voltage input is not depicted but only indicated by an orange arrow. $U_{0}$ is the AC voltage that is applied by the Solartron $1260 \mathrm{HV}$. The wire capacities $C_{0}, C_{1+}, C_{2+}$ and $C_{3+}$ are highlighted by green dashed rectangles.

Table 3-2: Values of some components of the experimental setup.

\begin{tabular}{|c|c|c|c|c|c|}
\hline $\boldsymbol{R}_{\mathbf{0}} / \boldsymbol{\Omega}$ & $\boldsymbol{R}_{\mathbf{1}} / \boldsymbol{\Omega}$ & $\boldsymbol{R}_{\mathbf{2}} / \boldsymbol{\Omega}$ & $\boldsymbol{C}_{\mathbf{0}} / \mathrm{F}$ & $\boldsymbol{C}_{\mathbf{1}} / \mathrm{F}$ & $\boldsymbol{C}_{\mathbf{2}} / \mathrm{F}$ \\
\hline $0-25 \cdot 10^{3}$ & $1 \cdot 10^{6}$ & $1 \cdot 10^{6}$ & $2.6 \cdot 10^{-10}$ & $1.0 \cdot 10^{-8}$ & $7.5 \cdot 10^{-9}$ \\
\hline
\end{tabular}

On the basis of this circuit diagram, expressions for the real and imaginary dielectric permittivity can be derived, depending on whether the voltage ratio $U_{2} / U_{1}$ or $U_{1} / U_{2}$ is determined:

$$
\begin{aligned}
& \varepsilon_{\mathrm{r}}^{\prime}=\frac{Z}{R_{0} C_{P}^{0}} \frac{x\left[\mathfrak{R}\left(\frac{U_{1}}{U_{2}}\right)-Z W\right]-y\left[\mathfrak{I}\left(\frac{U_{1}}{U_{2}}\right)-Z V\right]}{\left[\mathfrak{R}\left(\frac{U_{1}}{U_{2}}\right)-Z W\right]^{2}+\left[\mathfrak{I}\left(\frac{U_{1}}{U_{2}}\right)-Z V\right]^{2}} \\
& \varepsilon_{\mathrm{r}}^{\prime \prime}=-\frac{Z}{R_{0} C_{P}^{0}} \frac{y\left[\mathfrak{R}\left(\frac{U_{1}}{U_{2}}\right)-Z W\right]+x\left[\mathfrak{I}\left(\frac{U_{1}}{U_{2}}\right)-Z V\right]}{\left[\mathfrak{R}\left(\frac{U_{1}}{U_{2}}\right)-Z W\right]^{2}+\left[\mathfrak{I}\left(\frac{U_{1}}{U_{2}}\right)-Z V\right]^{2}} \\
& \varepsilon_{\mathrm{r}}^{\prime}=\frac{z}{R_{0} C_{P}^{0}} \frac{x \mathfrak{R}\left(\frac{U_{2}}{U_{1}}\right)+y \mathfrak{I}\left(\frac{U_{2}}{U_{1}}\right)+z(y v-x w)\left[\mathfrak{R}\left(\frac{U_{2}}{U_{1}}\right)^{2}+\mathfrak{I}\left(\frac{U_{2}}{U_{1}}\right)^{2}\right]}{1+2 z\left(v \mathfrak{I}\left(\frac{U_{2}}{U_{1}}\right)-W \Re\left(\frac{U_{2}}{U_{1}}\right)\right)+z^{2}\left(w^{2}+v^{2}\right)\left(\mathfrak{R}\left(\frac{U_{2}}{U_{1}}\right)^{2}+\mathfrak{I}\left(\frac{U_{2}}{U_{1}}\right)^{2}\right)}
\end{aligned}
$$




$$
\varepsilon_{\mathrm{r}}^{\prime \prime}=-\frac{z}{R_{0} C_{P}^{0}} \frac{y \mathfrak{R}\left(\frac{U_{2}}{U_{1}}\right)-x \mathfrak{I}\left(\frac{U_{2}}{U_{1}}\right)-z(y w+x V)\left(\mathfrak{R}\left(\frac{U_{2}}{U_{1}}\right)^{2}+\mathfrak{I}\left(\frac{U_{2}}{U_{1}}\right)^{2}\right)}{1+2 z\left(v \mathfrak{I}\left(\frac{U_{2}}{U_{1}}\right)-W \Re\left(\frac{U_{2}}{U_{1}}\right)\right)+z^{2}\left(v^{2}+W^{2}\right)\left(\mathfrak{R}\left(\frac{U_{2}}{U_{1}}\right)^{2}+\mathfrak{I}\left(\frac{U_{2}}{U_{1}}\right)^{2}\right)}
$$

The vacuum capacity $C_{P}{ }^{0}$ can be calculated from the area and distance of the electrodes of the sample capacitor. Further abbreviations are:

$$
\begin{gathered}
v=\left[R_{2}\left(C_{2}+C_{2+}\right)-R_{1}\left(C_{1}+C_{1+}\right)\right] \omega \\
w=1+R_{1} R_{2}\left(C_{1}+C_{1+}\right)\left(C_{2}+C_{2+}\right) \omega^{2} \\
x=R_{0}\left(C_{0}+C_{2}\right)+R_{2}\left(C_{2}+C_{2+}\right)-R_{1}\left(C_{1}+C_{1+}\right)+ \\
+R_{0} R_{1} R_{2}\left(C_{1}+C_{1+}\right)\left(C_{2} C_{2+}+C_{0} C_{2}+C_{0} C_{2+}\right) \omega^{2} \\
y=R_{1}\left(C_{1}+C_{1+}\right)\left(R_{0}\left(C_{0}+C_{2}\right)+R_{2}\left(C_{2}+C_{2+}\right)\right) \omega \\
z=\frac{R_{1} C_{1}}{R_{2} C_{2}} \frac{1}{1+R_{1}^{2}\left(C_{1}+C_{1+}\right)^{2} \omega^{2}}
\end{gathered}
$$

These expressions contain the wire capacities $C_{1+}$ and $C_{2+}$, which may vary after each sample exchange and are therefore unknown. They are, however, generally smaller than the capacities of the high-pass filters and the approximation $C_{1}, C_{2} \gg C_{1+}, C_{2+}$ leads to:

$$
\begin{gathered}
v \approx\left[R_{2} C_{2}-R_{1} C_{1}\right] \omega \\
w \approx 1+R_{1} R_{2} C_{1} C_{2} \omega^{2} \\
x \approx R_{0}\left(C_{0}+C_{2}\right)+R_{2} C_{2}-R_{1} C_{1}+R_{0} R_{1} R_{2} C_{1} C_{2}\left(C_{2+}+C_{0}\right) \omega^{2} \\
y \approx R_{1} C_{1}\left(R_{0}\left(C_{0}+C_{2}\right)+R_{2} C_{2}\right) \omega \\
z \approx \frac{R_{1} C_{1}}{R_{2} C_{2}} \frac{1}{1+R_{1}^{2} C_{1}^{2} \omega^{2}}
\end{gathered}
$$

The abbreviation $x$ still contains $C_{2+}$ though. Under the assumption that the sample capacity is much smaller than the wire capacities, $C_{2+}$ can be extracted from the resonance frequency at the maximum of $\mathfrak{I}\left(U_{2} / U_{1}\right)$ :

$$
\omega^{*}=\frac{1}{R_{0}\left(C_{2+}+C_{0}+\varepsilon^{\prime} C_{\mathrm{p}}^{0}\right)} \approx \frac{1}{R_{0}\left(C_{2+}+C_{0}\right)}
$$




$$
C_{2+}=\frac{1}{R_{0} \omega^{*}}-C_{0}
$$

This assumption is true for the present BMO samples at low-temperatures and therefore allows the determination of the real and imaginary dielectric permittivity at the multiferroic phase transition with equations (3-11)-(3-14). There is, however, another necessary correction of the data since the determined capacity of the sample capacitor $C=\varepsilon_{\mathrm{r}}{ }_{\mathrm{P}}{ }^{0}$ exhibits a constant offset $C_{\text {offset }}$ that is independent of the sample. This is probably due to the threat rods that hold the upper electrode in place (see Figure 3-10) and that are so close to both electrodes that they influence the electric field lines. This offset must be subtracted from the calculated sample capacity to obtain the correct relative permittivity:

$$
\varepsilon_{\mathrm{r}, \text { cor }}^{\prime}=\frac{C-C_{\text {offset }}}{C_{\mathrm{p}}^{0}}=\frac{\left(\varepsilon_{\mathrm{r}}^{\prime} C_{\mathrm{p}}^{0}\right)-C_{\text {offset }}}{C_{\mathrm{p}}^{0}}
$$

The permittivity of BMO drastically increases above $150 \mathrm{~K}$ due to a number of anisotropic, dielectric relaxations with Debye-character. In order to investigate these, it is necessary to determine the real and imaginary permittivity for a wide range of frequencies. The high-pass filters, however, place a lower limit of $\omega \approx 100 \mathrm{~Hz}$ to the accessible frequencies, which greatly reduces the data quality because some of these relaxations take place on a larger time scale. In order the expand the range down to $1 \mathrm{~Hz}$, the high-voltage decoupling device is replaced by a similar device without high-pass filters, whose circuit diagram is displayed in Figure 3-9.

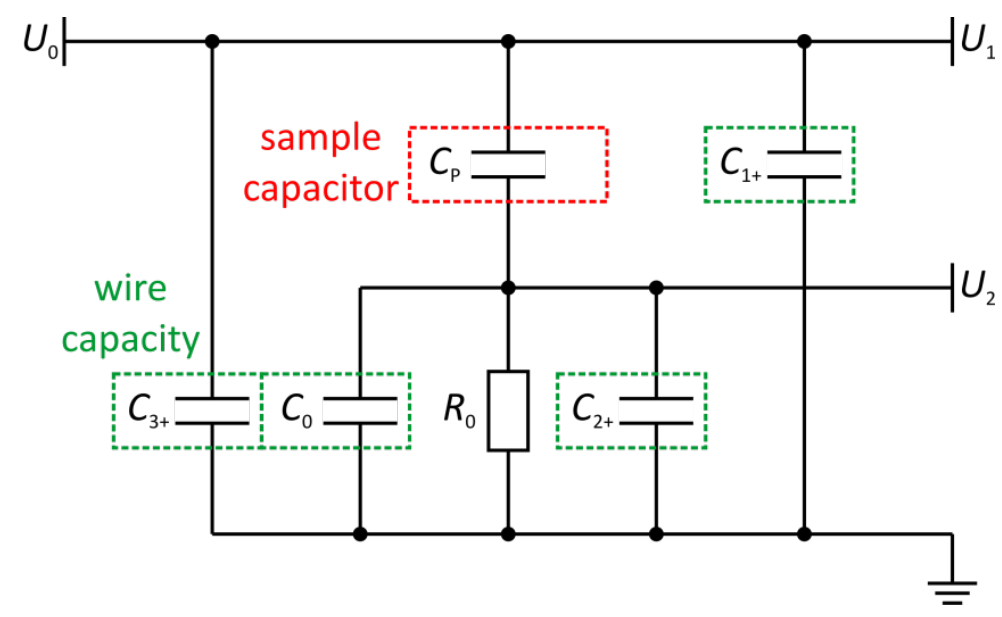

Figure 3-13: Circuit diagram of the setup without high voltage decoupling device.

For this setup, the equations for the calculations of the dielectric permittivity are much simpler: 


$$
\begin{aligned}
& \varepsilon_{\mathrm{r}}^{\prime}=\frac{1}{R_{0} C_{P}^{0}} \frac{R_{0}\left(C_{0}+C_{2+}\right)\left[\mathfrak{R}\left(\frac{U_{1}}{U_{2}}\right)-1\right]-\frac{1}{\omega} \mathfrak{I}\left(\frac{U_{1}}{U_{2}}\right)}{\left[\Re\left(\frac{U_{1}}{U_{2}}\right)-1\right]^{2}+\left[\mathfrak{J}\left(\frac{U_{1}}{U_{2}}\right)\right]^{2}} \\
& \varepsilon_{\mathrm{r}}^{\prime \prime}=-\frac{1}{R_{0} C_{P}^{0}} \frac{\frac{1}{\omega}\left[\Re\left(\frac{U_{1}}{U_{2}}\right)-1\right]+R_{0}\left(C_{0}+C_{2+}\right) \Im\left(\frac{U_{1}}{U_{2}}\right)}{\left[\Re\left(\frac{U_{1}}{U_{2}}\right)-1\right]^{2}+\left[\mathfrak{I}\left(\frac{U_{1}}{U_{2}}\right)\right]^{2}} \\
& \varepsilon_{\mathrm{r}}^{\prime}=\frac{1}{R_{0} C_{P}^{0}} \frac{R_{0}\left(C_{0}+C_{2+}\right) \Re\left(\frac{U_{2}}{U_{1}}\right)+\frac{1}{\omega} \mathfrak{J}\left(\frac{U_{2}}{U_{1}}\right)-R_{0}\left(C_{0}+C_{2+}\right)\left[\mathfrak{R}\left(\frac{U_{2}}{U_{1}}\right)^{2}+\mathfrak{I}\left(\frac{U_{2}}{U_{1}}\right)^{2}\right]}{\left[\mathfrak{R}\left(\frac{U_{2}}{U_{1}}\right)-1\right]^{2}+\mathfrak{I}\left(\frac{U_{2}}{U_{1}}\right)^{2}} \\
& \varepsilon_{\mathrm{r}}^{\prime \prime}=-\frac{1}{R_{0} C_{P}^{0}} \frac{\frac{1}{\omega} \mathfrak{R}\left(\frac{U_{2}}{U_{1}}\right)-R_{0}\left(C_{0}+C_{2+}\right) \mathfrak{I}\left(\frac{U_{2}}{U_{1}}\right)-\frac{1}{\omega}\left(\mathfrak{R}\left(\frac{U_{2}}{U_{1}}\right)^{2}+\mathfrak{I}\left(\frac{U_{2}}{U_{1}}\right)^{2}\right)}{\left[\mathfrak{R}\left(\frac{U_{2}}{U_{1}}\right)-1\right]^{2}+\mathfrak{J}\left(\frac{U_{2}}{U_{1}}\right)^{2}}
\end{aligned}
$$

Nevertheless, the wire capacity $C_{2+}$ must still be determined via the resonance frequency as explained above.

\subsubsection{Debye relaxation}

Debye-relaxations describe the time-dependent response of permanent dipoles to an alternating electric field with angular frequency $\omega$. The resulting electric polarization is divided in two contributions. An instantaneous response at $t=0$ is obtained even for infinite frequencies and therefore called $P_{\infty}$. The static response $P_{s}$, on the other hand, is the saturation polarization that is only reached for $t \rightarrow \infty$. The difference $\Delta P=P_{\mathrm{s}}-P_{\infty}$ is the contribution that develops over time when the dipoles align with the external field:

$$
P(t)=P_{\infty}+\Delta P(1-\exp (-t / \tau))
$$

The relaxation time $\tau$ is the parameter that describes the time-dependence of the overall polarization $P(\mathrm{t})$. Both contributions of $P$ can be expressed in terms of the dielectric permittivity $\varepsilon_{\infty}$ and $\Delta \varepsilon$. Thus, for the frequency-dependence of the complex permittivity follows [115]:

$$
\varepsilon(\omega)=\varepsilon^{\prime}(\omega)+i \varepsilon^{\prime \prime}(\omega)=\varepsilon_{\infty}+\frac{\Delta \varepsilon}{1+(i \omega \tau)^{\beta}}
$$

$\beta$ is the damping factor that describes the dispersion behavior of the permittivity. Separation of the real and imaginary part yields the Cole-Cole-equations: 


$$
\begin{gathered}
\varepsilon^{\prime}(\omega)=\varepsilon_{\infty}+\Delta \varepsilon \frac{1+\cos \left(\beta \frac{\pi}{2}\right)(\omega \tau)^{\beta}}{1+(\omega \tau)^{2 \beta}+2 \cos \left(\beta \frac{\pi}{2}\right)(\omega \tau)^{\beta}} \\
\varepsilon^{\prime \prime}(\omega)=-\Delta \varepsilon \frac{\sin \left(\beta \frac{\pi}{2}\right)(\omega \tau)^{\beta}}{1+(\omega \tau)^{2 \beta}+2 \cos \left(\beta \frac{\pi}{2}\right)(\omega \tau)^{\beta}}
\end{gathered}
$$

The common Debye-equations are a special case where $\beta=1$ :

$$
\begin{gathered}
\varepsilon^{\prime}(\omega)=\varepsilon_{\infty}+\frac{\Delta \varepsilon}{1+(\omega \tau)^{2}} \\
\varepsilon^{\prime \prime}(\omega)=-\frac{\Delta \varepsilon \omega \tau}{1+(\omega \tau)^{2}}
\end{gathered}
$$

\subsection{Determination of elastic constants}

Elastic constants are the proportionality constants of the generalized Hooke's law that describes the relationship between mechanical stress $\sigma_{\mathrm{ik}}$ and elastic deformation $\varepsilon_{\mathrm{m}}$ :

$$
\boldsymbol{\sigma}=\boldsymbol{C} \boldsymbol{\varepsilon} \quad \sigma_{\mathrm{ik}}=C_{\mathrm{ik}, \mathrm{lm}} \varepsilon_{\mathrm{lm}}
$$

$\sigma$ and $\varepsilon$ are symmetric second rank tensors which each have six independent components. $\boldsymbol{C}$ is the fourth rank tensor of elastic constants. The inverse Hooke's law contains another set of parameters $\boldsymbol{S}$ that is called compliance tensor:

$$
\varepsilon=S \sigma \quad S=C^{-1}
$$

In fact, $\boldsymbol{S}$ is a part of Schmid's power series that describes the contributions of higher order ferroics to the free energy (see equation (2-5) in chapter 2.1.2). Specifically, it is the uniform second order contribution of the external stress field. The compliance tensor itself is the inverse of $\boldsymbol{C}$. Consequently, if the elastic constants of a material are known, the mechanical behavior can be predicted. Especially, possible alterations during the transition into a multiferroic phase can provide evidence about the question if the multiferroic coupling mechanism has a magnetoelastic component.

The elastic constants are, however, experimentally not directly accessible and common methods like ultrasound measurements or inelastic neutron scattering rather determine the velocity 
of sound waves $v_{\mathrm{s}}$. $\boldsymbol{C}$ and $v_{\mathrm{s}}$ are connected via the propagation tensor $\Gamma$, whose components can be described as:

$$
\Gamma_{\mathrm{im}}=\sum_{\mathrm{k}, \mathrm{n}} C_{\mathrm{ik}, \mathrm{mn}} \frac{q_{\mathrm{k}} q_{\mathrm{n}}}{|\boldsymbol{q}|^{2}}
$$

$\Gamma$ is a function of the propagation vector $\boldsymbol{q}$ of the sound wave since each component of $\Gamma$ is a linear combination of different $C_{\mathrm{ik}, \mathrm{mn}}$ whose linear coefficients depend on the propagation direction. For a given $\boldsymbol{q}$, the eigenvalue equation of $\Gamma$ can be described as:

$$
\rho v_{s}^{2} u=\Gamma u
$$

where $\rho$ is the density of the material and $u$ is the polarization vector which describes the atomic displacement direction of a sound wave. There are three different eigenvector solutions $\boldsymbol{u}$ for every propagation direction with different eigenvalues and therefore different $v_{s}$, each depending on a certain combination of $C_{i k, m n}$. In order to determine all possible elastic constants, the velocities of a certain number of different sound waves must be measured. The minimum number strongly depends on the symmetry of the material. Since the elastic constants of orthorhombic BMO are determined in this thesis, these considerations shall be exemplified with orthorhombic symmetry.

For the purpose of a better visualization, it is common to use Voigt's notation where the components of $\sigma$ and $\varepsilon$ are represented by a six-dimensional vector. Instead of the four-rank tensor $\boldsymbol{C}$, the elastic constants are represented by a $6 \times 6$-matrix $c$. Please note the different notation with capital and small letter, respectively. The number of independent components of $c$ depends on the symmetry of the material. In the orthorhombic case, there are 9 components:

$$
c_{\text {ort }}=\left(\begin{array}{cccccc}
c_{11} & c_{12} & c_{13} & 0 & 0 & 0 \\
c_{12} & c_{22} & c_{23} & 0 & 0 & 0 \\
c_{13} & c_{23} & c_{33} & 0 & 0 & 0 \\
0 & 0 & 0 & c_{44} & 0 & 0 \\
0 & 0 & 0 & 0 & c_{55} & 0 \\
0 & 0 & 0 & 0 & 0 & c_{66}
\end{array}\right)
$$

In a first step, the propagation tensor for sound waves that propagate along the principal directions shall be considered. They are listed in Table 3-3 together with their respective eigenvectors and eigenvalues. For these particular cases, there is one solution where $\boldsymbol{u}$ is parallel to $\boldsymbol{q}$ and the atoms are therefore displaced along the propagation direction of the sound wave. These waves are called longitudinal (LA) in contrast to the two transverse waves $\left(T_{1} A\right.$ and $\left.T_{2} A\right)$ where $\boldsymbol{u}$ is perpendicular to $\boldsymbol{q}$. Each sound velocity is connected to only one elastic constant. In fact, all diagonal 
elements of the $c$-matrix can be determined with sound waves along these three directions and three elastic constants are even doubly redundant.

Table 3-3: Propagation tensor $\Gamma$ for sound waves that propagate along the principal axes. $u$ and $\rho v_{s}$ are the eigenvectors and eigenvalues of $\Gamma$. For each propagation direction $\boldsymbol{q}$, there is one longitudinal (LA) and two transverse $\left(T_{1} A\right.$ and $\left.T_{2} A\right)$ sound waves.

\begin{tabular}{|c|cc|c|c|c|c|c|c|}
\hline $\boldsymbol{q}$ & $\Gamma$ & $\boldsymbol{u}_{\mathrm{LA}}$ & $\boldsymbol{u}_{\mathrm{T} 1 \mathrm{~A}}$ & $\boldsymbol{u}_{\mathrm{T} 2 \mathrm{~A}}$ & $\rho v_{\mathrm{s}, \mathrm{LA}}$ & $\rho v_{\mathrm{s}, \mathrm{T} \mathrm{A}}$ & $\rho v_{\mathrm{s}, \mathrm{T} 2 \mathrm{~A}}$ \\
\hline$\left(\begin{array}{l}\xi \\
0 \\
0\end{array}\right)$ & $\left(\begin{array}{ccc}c_{11} & 0 & 0 \\
0 & c_{66} & 0 \\
0 & 0 & c_{55}\end{array}\right)$ & $\left(\begin{array}{l}1 \\
0 \\
0\end{array}\right)$ & $\left(\begin{array}{l}0 \\
1 \\
0\end{array}\right)$ & $\left(\begin{array}{l}0 \\
0 \\
1\end{array}\right)$ & $c_{11}$ & $c_{66}$ & $c_{55}$ \\
\hline$\left(\begin{array}{l}0 \\
\xi \\
0\end{array}\right)$ & $\left(\begin{array}{ccc}c_{66} & 0 & 0 \\
0 & c_{22} & 0 \\
0 & 0 & c_{44}\end{array}\right)$ & $\left(\begin{array}{l}0 \\
1 \\
0\end{array}\right)$ & $\left(\begin{array}{l}1 \\
0 \\
0\end{array}\right)$ & $\left(\begin{array}{l}0 \\
0 \\
1\end{array}\right)$ & $c_{22}$ & $c_{66}$ & $c_{44}$ \\
\hline$\left(\begin{array}{l}0 \\
0 \\
\xi\end{array}\right)$ & $\left(\begin{array}{ccc}c_{55} & 0 & 0 \\
0 & c_{44} & 0 \\
0 & 0 & c_{33}\end{array}\right)$ & $\left(\begin{array}{l}0 \\
0 \\
1\end{array}\right)$ & $\left(\begin{array}{l}1 \\
0 \\
0\end{array}\right)$ & $\left(\begin{array}{l}0 \\
1 \\
0\end{array}\right)$ & $c_{33}$ & $c_{55}$ & $c_{44}$ \\
\hline
\end{tabular}

In order to access the remaining three off-diagonal elements of $\boldsymbol{c}$, other directions of $\boldsymbol{q}$ must be considered. Table 3-4 lists the propagation tensors for sound waves that propagate in between two principal axes as denoted in the first column. In each case, there is one trivial eigenvector solution where $\boldsymbol{u}$ is perpendicular to $\boldsymbol{q}$ and parallel to one principal axis. Unfortunately, the corresponding eigenvalue of this transverse wave does not contain any of the desired off-diagonal elements. Therefore, the remaining two-dimensional subspace has to be inspected. Each propagation direction yields one off-diagonal element.

Table 3-4: Propagation tensor $\Gamma$ for sound waves that propagate along different directions $\boldsymbol{q}$ in between two principal axes. $\alpha, \beta$ and $\gamma$ are ratios of lattice parameter and listed in the last column.

\begin{tabular}{|c|c|c|c|}
\hline $\boldsymbol{q}$ & $\Gamma$ & \\
\hline$\left(\begin{array}{l}\xi \\
\xi \\
0\end{array}\right)$ & $\frac{1}{1+\gamma^{2}}\left(\begin{array}{ccc}c_{11}+\gamma^{2} c_{66} & \gamma^{2}\left(c_{12}+c_{66}\right) & 0 \\
\gamma^{2}\left(c_{12}+c_{66}\right) & c_{66}+\gamma^{2} c_{22} & 0 \\
0 & 0 & c_{55}+\gamma^{2} c_{44}\end{array}\right)$ & $\gamma=\frac{a}{b}$ \\
\hline$\left(\begin{array}{l}\xi \\
0 \\
\xi\end{array}\right)$ & $\frac{1}{1+\beta^{2}}\left(\begin{array}{ccc}c_{55}+\beta^{2} c_{11} & 0 & \beta\left(c_{13}+c_{55}\right) \\
0 & c_{44}+\beta^{2} c_{66} & 0 \\
\beta\left(c_{13}+c_{55}\right) & 0 & c_{33}+\beta^{2} c_{55}\end{array}\right)$ & $\beta=\frac{c}{a}$ \\
\hline$\left(\begin{array}{l}0 \\
\xi \\
\xi\end{array}\right)$ & $\frac{1}{1+\alpha^{2}}\left(\begin{array}{ccc}c_{66}+\alpha^{2} c_{55} & 0 & 0 \\
0 & c_{22}+\alpha^{2} c_{44} & \alpha\left(c_{23}+c_{44}\right) \\
0 & \alpha\left(c_{23}+c_{44}\right) & c_{44}+\alpha^{2} c_{33}\end{array}\right)$ & $\alpha=\frac{b}{c}$ \\
\hline
\end{tabular}


The remaining eigenvalues for $\Gamma(\xi, \xi, 0)$ as an example are:

$$
\rho v_{s \pm}^{2}=\frac{1}{1+\gamma^{2}}\left[\begin{array}{l}
1 / 2\left(c_{11}+c_{66}+\gamma^{2}\left(c_{66}+c_{22}\right)\right) \pm \\
\pm \sqrt{\gamma^{2}\left(c_{12}+c_{66}\right)^{2}+1 / 4\left(c_{11}-c_{66}+\gamma^{2}\left(c_{66}-c_{22}\right)\right)^{2}}
\end{array}\right]
$$

If all diagonal elastic constant are determined beforehand, it is sufficient to measure the velocity of only one of the two sound waves in order to calculate $c_{12}$. The corresponding eigenvectors are:

$$
\boldsymbol{u}_{ \pm}=\frac{1}{\sqrt{\left(\rho v_{s \pm}^{2}-c_{66}-\gamma^{2} c_{22}\right)^{2}+\gamma^{2}\left(c_{12}+c_{66}\right)^{2}}}\left(\begin{array}{c}
\rho v_{s \pm}^{2}-c_{66}-\gamma^{2} c_{22} \\
\gamma\left(c_{12}+c_{66}\right) \\
0
\end{array}\right)
$$

Both polarization vectors depend on the actual values of the elastic constants and they are not necessarily parallel or perpendicular to the propagation vector. Therefore, the terminology "Iongitudinal" and "transverse" cannot be applied. In the case of BMO, both eigenvectors can be calculated from the elastic constants that are presented in chapter 6.4.2. They are in fact nearly parallel or perpendicular to $\boldsymbol{q}$, respectively, and further on called "pseudo-longitudinal" and "pseudotransverse". For the sake of simplicity, the labels $L A$ and $T_{1} A$ are kept. The wave corresponding to the trivial eigenvector $\left(T_{2} A\right)$, on the other hand, is perfectly transverse. For the other two propagation directions, similar expressions like equation (3-43) and (3-44) can be derived. For $\Gamma(\xi, 0, \xi)$ :

$$
\begin{aligned}
& \rho v_{s \pm}^{2}=\frac{1}{1+\beta^{2}}\left[\begin{array}{l}
1 / 2\left(c_{55}+c_{33}+\beta^{2}\left(c_{11}+c_{55}\right)\right) \pm \\
\pm \sqrt{\beta^{2}\left(c_{13}+c_{55}\right)^{2}+1 / 4\left(c_{55}-c_{33}+\beta^{2}\left(c_{11}-c_{55}\right)\right)^{2}}
\end{array}\right] \\
& \boldsymbol{u}_{ \pm}=\frac{1}{\sqrt{\left(\rho v_{s \pm}^{2}-c_{33}-\beta^{2} c_{55}\right)^{2}+\beta^{2}\left(c_{13}+c_{55}\right)^{2}}}\left(\begin{array}{c}
\rho v_{s \pm}^{2}-c_{33}-\beta^{2} c_{55} \\
0 \\
\beta\left(c_{13}+c_{55}\right)
\end{array}\right)
\end{aligned}
$$

and for $\Gamma(0, \xi, \xi)$ :

$$
\begin{aligned}
& \rho v_{s \pm}^{2}=\frac{1}{1+\alpha^{2}}\left[\begin{array}{l}
1 / 2\left(c_{22}+c_{44}+\alpha^{2}\left(c_{44}+c_{33}\right)\right) \pm \\
\pm \sqrt{\alpha^{2}\left(c_{23}+c_{44}\right)^{2}+1 / 4\left(c_{22}-c_{44}+\alpha^{2}\left(c_{44}-c_{33}\right)\right)^{2}}
\end{array}\right] \\
& \boldsymbol{u}_{ \pm}=\frac{1}{\sqrt{\left(\rho v_{s \pm}^{2}-c_{44}-\alpha^{2} c_{33}\right)^{2}+\alpha^{2}\left(c_{23}+c_{44}\right)^{2}}}\left(\begin{array}{c}
0 \\
\rho v_{s \pm}^{2}-c_{44}-\alpha^{2} c_{33} \\
\alpha\left(c_{23}+c_{44}\right)
\end{array}\right)
\end{aligned}
$$


In summary, the measuring strategy consists of two consecutive steps. First, the velocities of at least six different sound waves that propagate along the principal axes of the material have to be measured. Among them are the three longitudinal waves and a reasonable combination of three transverse ones. This allows the calculation of all diagonal elastic constants. Second, the velocity of the pseudo-longitudinal or pseudo-transverse wave for each of the propagation directions in between two principal axes has to be determined. For every direction, one of the remaining off-diagonal elements can be calculated. 


\section{Synthesis and Characterization of Mo-doped $\mathrm{MnWO}_{4}$}

There are several publications that demonstrate that the magnetic phase transition temperatures $T_{\mathrm{N} 2}$ and $T_{\mathrm{N} 3}$ of $\mathrm{MnWO}_{4}$ increase when tungsten atoms are replaced by molybdenum $[18,63,64]$ (for more details see chapter 2.3.1.5). This is of special interest because most type-II multiferroics, like $\mathrm{MnWO}_{4}$, are multiferroic at temperatures that are too low for any feasible widespread application. Any increase of this temperature might therefore help to reach the goal of finding room temperature multiferroics.

The phase diagram of the system $\mathrm{MnW}_{1-x} \mathrm{Mo}_{x} \mathrm{O}_{4}$ is, however, not very well understood. Since $\mathrm{MnWO}_{4}(x=0)$ has a different crystal structure than $\mathrm{MnMoO}_{4}(x=1)$, it is not possible to continuously solve arbitrary amounts of molybdenum in $\mathrm{MnWO}_{4}$ for the whole range of $0<x<1$. Instead, the system must have a miscibility gap with lower and upper limits $x_{1}$ and $x_{\mathrm{u}}$, respectively, where two phases coexist with constant compositions of $\mathrm{MnW}_{1-x \mid} \mathrm{Mo}_{x \mid} \mathrm{O}_{4}$ and $\mathrm{MnW}_{1-x u} \mathrm{Mo}_{x u} \mathrm{O}_{4}$, which are further on called wolframite and molybdate phase, respectively. Only the phase fraction $f_{\mathrm{W}}$ and $f_{\mathrm{M}}$ of both phases vary with $x$ according to:

$$
f_{\mathrm{W}}=\frac{x_{\mathrm{u}}-x}{x_{\mathrm{u}}-x_{\mathrm{l}}} \quad f_{\mathrm{M}}=1-x_{\mathrm{w}}
$$

The limits of the miscibility gap are still under debate, though. Chang determined a phase diagram by temperature dependent X-ray powder diffraction where the lower and upper limit varies from $600^{\circ} \mathrm{C}$ to $1050^{\circ} \mathrm{C}$ in the range of $0.1<x_{1}<0.15$ and $0.9>x_{1}>0.7$. respectively [69]. This is contradicted by Meddar who synthesized a sample with $x=0.3$ that only consists of wolframite phase at $1100^{\circ} \mathrm{C}$ [18]. A weak Bragg-reflection of the molybdate phase first appears in the diffraction pattern of the $x=0.4$ sample. This discrepancy might be explained by different ways of synthesis that are employed by Chang and Meddar. Hardy, on the other hand, prepared samples by the same synthesis route like Meddar and found signs of the molybdate phase by neutron powder diffraction already for the $x=0.2$ sample [63], which is again in agreement with Chang despite the different synthesis. Blanco-Gutierrez employed a third way of synthesis at lower temperatures that involves extensive ball-milling after the reaction and achieved to solve even $50 \%$ molybdenum in the wolframite phase [71].

In order to clarify these contradictions, this chapter of the thesis aims to characterize the miscibility gap of the system $\mathrm{MnW}_{1-\mathrm{x}} \mathrm{Mo}_{x} \mathrm{O}_{4}$. Samples in the range of $0<x<1$ are synthesized according to Meddar and characterized by X-ray powder diffraction and Raman spectroscopy. Furthermore, ferroelectric and magnetic phase transition temperatures are determined by the measurement of the dielectric permittivity and magnetic susceptibility. 
The synthesis is explained in detail in the first subchapter, while the second one lists all relevant experimental parameters of the dielectric measurements. The experimental results are presented and discussed in the third subchapter and conclusively summarized in the fourth and last subchapter.

\subsection{Synthesis}

Two different batches of Mo-doped $\mathrm{MnWO}_{4}$ were prepared during this thesis. They differ in some characteristics of the synthesis like temperature and duration, but the overall procedure is the same and was initially described by [18]. This chapter will focus on the general aspects while all parameters that differ between both batches are listed in Table 4-1. The reasons for the variances of individual batches will be discussed together with experimental results in the respective subsections of chapter 4.3 .

Starting materials for the synthesis are powders of the mono-metallic oxides: MnO $99 \%$ by ChemPur $\mathrm{GmbH}, \mathrm{MoO}_{3} 99.5 \%$ by Thermo Fischer $\mathrm{GmbH}$ and $\mathrm{WO}_{3} 99 \%$ by ChemPur $\mathrm{GmbH}$. They were mixed in stoichiometric ratio and homogenized with the planetary ball mill Pulverisette 6 by Fritsch $\mathrm{GmbH}$. A grinding beaker out of agate was used together with 5 agate balls with a diameter of $20 \mathrm{~mm}$. The rotation frequency was $300 \mathrm{~min}^{-1}$ while the milling duration $t_{\text {mil }}$ varied between batches.

The homogenized powder was pressed to cylindrical pellets with a hydraulic press by Paul Weber $\mathrm{GmbH}$. These pellets have a diameter of $10 \mathrm{~mm}$ but varied in height. They were placed in a muffle furnace where the actual reaction took place. The temperature $T_{\text {syn }}$ and duration $t_{\text {syn }}$ of the synthesis varied between batches. The pellets were afterwards crushed in a mortar and milled again in order to check the reaction progress by XRPD. The whole cycle of pressing, reaction and milling was repeated until no Bragg-reflections of the starting material were detectable anymore. The number of necessary cycles $N_{\text {cyc }}$ depends on $t_{\text {mil, }} T_{\text {syn }}$ and $t_{\text {syn }}$ and therefore varies between both batches. After completed synthesis, some pellets of the first and second batch were sintered in the muffle furnace at a temperature $T_{\text {sint }}=1100^{\circ} \mathrm{C}$ for different durations $t_{\text {sint }}$.

Table 4-1: Milling time $t_{\text {mil, }}$ temperature $T_{\text {syn }}$ and duration $t_{\text {syn }}$ of the synthesis, number of reaction cycles $N_{\text {cyc }}$, temperature $T_{\text {sint }}$ and duration $t_{\text {sint }}$ of the sintering process for all three batches.

\begin{tabular}{|c|c|c|c|c|c|c|}
\hline batch $^{\mathbf{o}}$ & $\boldsymbol{t}_{\text {mil }} / \mathbf{m i n}$ & $\boldsymbol{T}_{\text {syn }} /{ }^{\circ} \mathbf{C}$ & $\boldsymbol{t}_{\text {syn }} / \mathbf{h}$ & $\boldsymbol{N}_{\text {cyc }}$ & $\boldsymbol{T}_{\text {sint }} /{ }^{\circ} \mathbf{C}$ & $\boldsymbol{t}_{\text {sint }} / \mathbf{h}$ \\
\hline 1 & $5-15$ & 800 & $48-72$ & 2 & 1100 & $48-72$ \\
\hline 2 & 60 & 600 & 168 & $2-3$ & 1100 & 66 \\
\hline
\end{tabular}




\subsection{Instrumental setup}

All experimental parameters that are specific for the present dielectric investigations are listed in this chapter while the basic principles of this method are described in chapter 3.2. The instrumental setup with high voltage decoupling device was used for all measurements of Mo-doped $\mathrm{MnWO}_{4}$. The frequency of the external electric field ranges from $100 \mathrm{~Hz}$ to $1 \mathrm{MHz}$. Impedance spectra were collected while the temperature of the sample was continuously increased or decreased by a rate of $0.1-0.3 \mathrm{~K} \mathrm{~min}^{-1}$ in the temperature range of $7.5-25 \mathrm{~K}$. Several cycles of heating and cooling were repeated for each sample. 


\subsection{Results and discussion}

The characteristics of all synthesized samples are described in this chapter in order to determine the phase diagram of the system $\mathrm{MnW}_{1-x} \mathrm{Mo}_{x} \mathrm{O}_{4}$. The first subchapter explains how the diffraction patterns are evaluated using Rietveld-refinement. Samples of the first batch are characterized on the basis of X-ray and Raman results in the second subchapter. Conclusions from these results lead to the synthesis of the second batch, which is discussed in the third subchapter. Finally, the determination of phase transition temperatures by measurements of the dielectric permittivity and magnetic susceptibility is presented in the fourth and fifth subchapter, respectively.

\subsubsection{Evaluation of X-ray powder diffraction pattern}

The reaction progress during synthesis as well as the characteristics of the final samples is determined by X-ray powder diffraction. This chapter will therefore focus on the evaluation of diffraction patterns while the basic principle XRPD is explained in chapter 3.1.1. An exemplary pattern of sintered $\mathrm{MnWO}_{4}$ of the first batch is presented as red line in Figure 4-1. All diffraction patterns were collected in a range of scattering angles between $15^{\circ}$ and $60^{\circ}$. The characteristic Bragg-reflections lie on a background, which is marked by a blue dashed line. For a better presentation and comparison of sample relevant information, the background intensity of all diffraction patterns was determined and subtracted by a B-spline interpolation of several points in between Bragg-reflections. The result of the background subtraction is displayed as black line in Figure 4-1. Please note that there are two reflections of the tantalum sample holder at $38.5^{\circ}$ and $55.6^{\circ}$, which are labeled by "Ta".

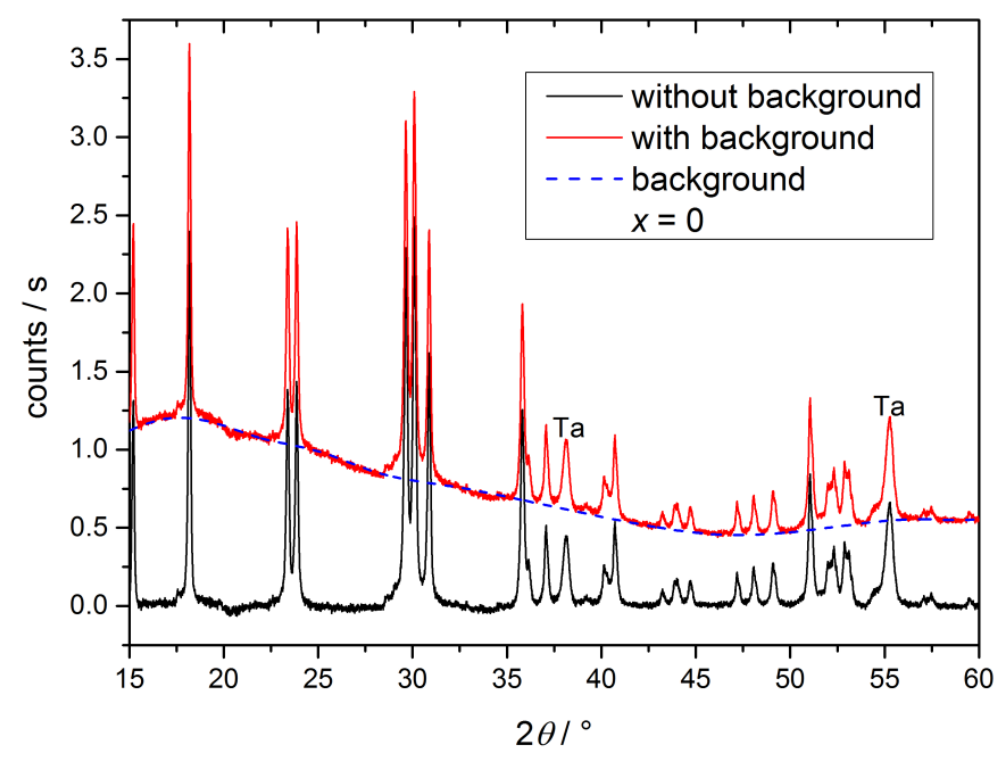

Figure 4-1: Effect of the background subtraction. The red line is the diffraction pattern of sintered $\mathrm{MnWO}_{4}$ of the first batch while the black line is the same pattern with subtracted background which is in turn represented by a blue dashed line. Both reflections of the tantalum sample holder are labelled by "Ta". 
In order to investigate the miscibility gap, it is necessary to determine the relative intensity that the wolframite and molybdate phase contribute to the overall diffraction pattern, which in turn depends on their phase fraction $f_{\mathrm{W}}$ and $f_{\mathrm{M}}$, respectively. The method of choice for a quantitative analysis of powder samples is Rietveld refinement. A theoretical diffraction pattern is calculated on the basis of several parameters like the instrumental resolution function, correction parameters for the experimental setup like sample displacement, lattice parameters of the sample, texture, grain size and, most important for this thesis, the phase fraction. Some of these parameters are then varied by a least-squares fit to match the experimental diffraction pattern. The software package "GSASII v.0.2.0" [116] was used during this thesis.
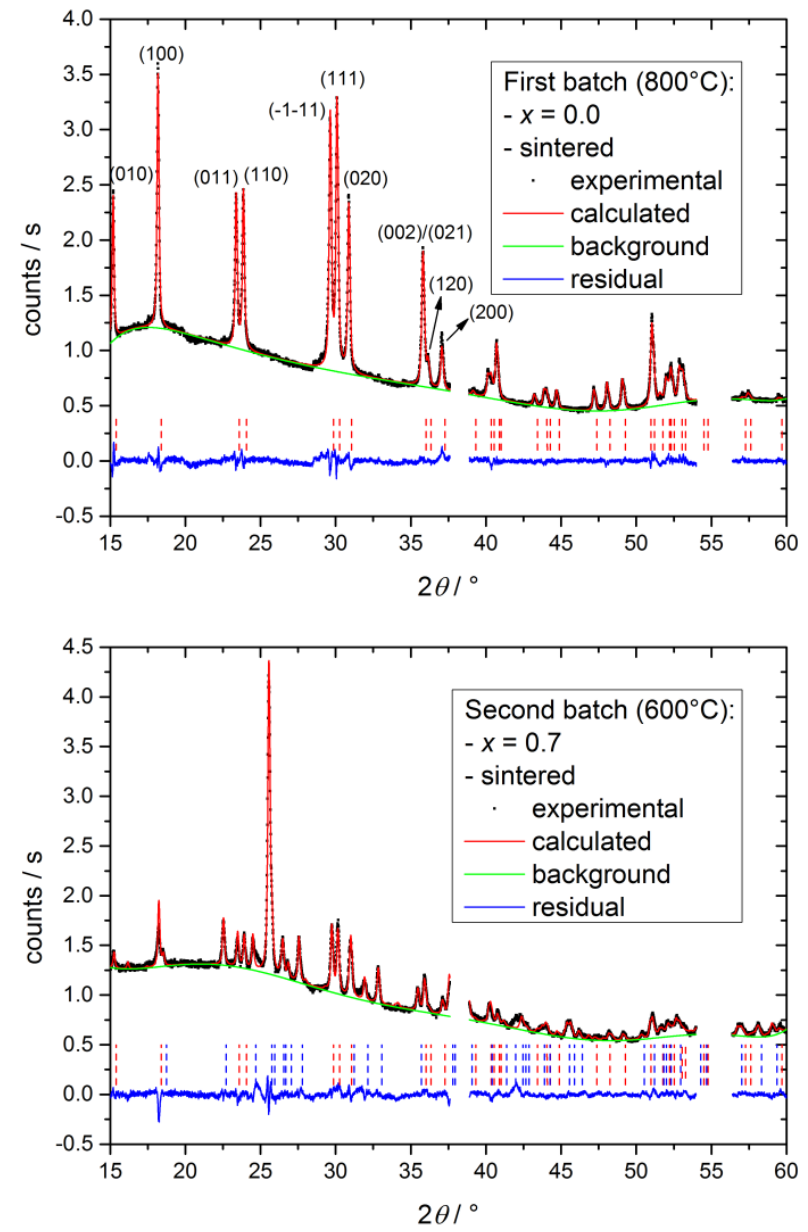

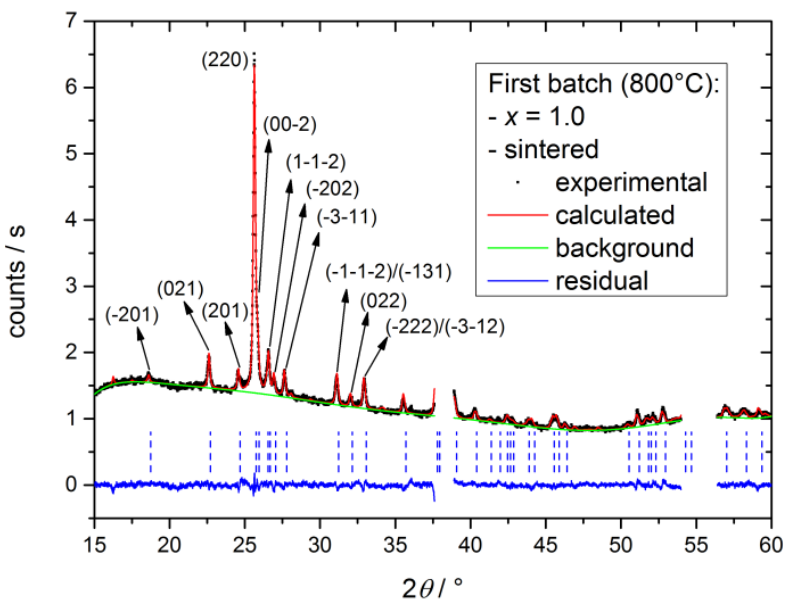

Figure 4-2: Rietveld refinement of three different sintered samples as examples. Black dots are experimental data points while the calculated pattern is represented as a red line. The green line is the background and the blue line is residual intensity. Red and blue dashed lines are calculated peak positions of the wolframite and molybdate phase, respectively. Some relevant reflections are labelled. The Bragg-reflections of the tantalum sample holder are excluded from refinement.

Three examples of sintered samples with $x=0.0,0.7$ and 1.0 are presented in Figure 4-2. Black dots are experimental data points while the calculated pattern is represented as a red line. Red and blue dashed lines are calculated peak positions of the wolframite and molybdate phase, respectively. The diffraction pattern of pure $\mathrm{MnMoO}_{4}$ is significantly different than the $\mathrm{MnWO}_{4}$-pattern, since the (220)-Bragg reflection dominates the whole diffractogram while all other reflections have much less intensity. 
In a first step, the instrumental resolution function that defines the full width at half maximum of a delta-signal as function of the scattering angle was determined by a diffraction pattern of $\mathrm{LaB}_{6}$. The parameters of this function were then set constant and used for the refinement of the sample patterns. The published crystal structures of pure $\mathrm{MnWO}_{4}$ and $\mathrm{MnMoO}_{4}[38,68]$ serve as starting point for the refinement. The background is simulated by a polynomial of $10^{\text {th }}$ order. As first refinement step, the parameters of the background, a general scaling factor, an offset of the $x$-axis and the phase fraction are varied. In the second step, texturing parameters are introduced, because the relative intensities of several Bragg-reflections differ significantly for many sintered samples, which is exemplified by pure $\mathrm{MnWO}_{4}$ of the first batch in Figure 4-3. This is a clear indicator that individual grains of the powder are not randomly oriented during measurement, but have a preferred direction. Texturing is simulated by spherical harmonics of $8^{\text {th }}$ and $4^{\text {th }}$ order for the wolframite and molybdate phase, respectively. This effect is most pronounced for reflections with predominant $k$-component in the wolframite phase.

The lattice parameters are refined in the third step and isotropic models for domain size and microstrain are applied in the final fourth step. The latter only serve as tool for a further adjustment of the peak profiles in addition to the instrumental resolution function. In the end, the phase fraction and lattice parameters of the wolframite phase are essential for the characterization of the miscibility gap in the following chapters.

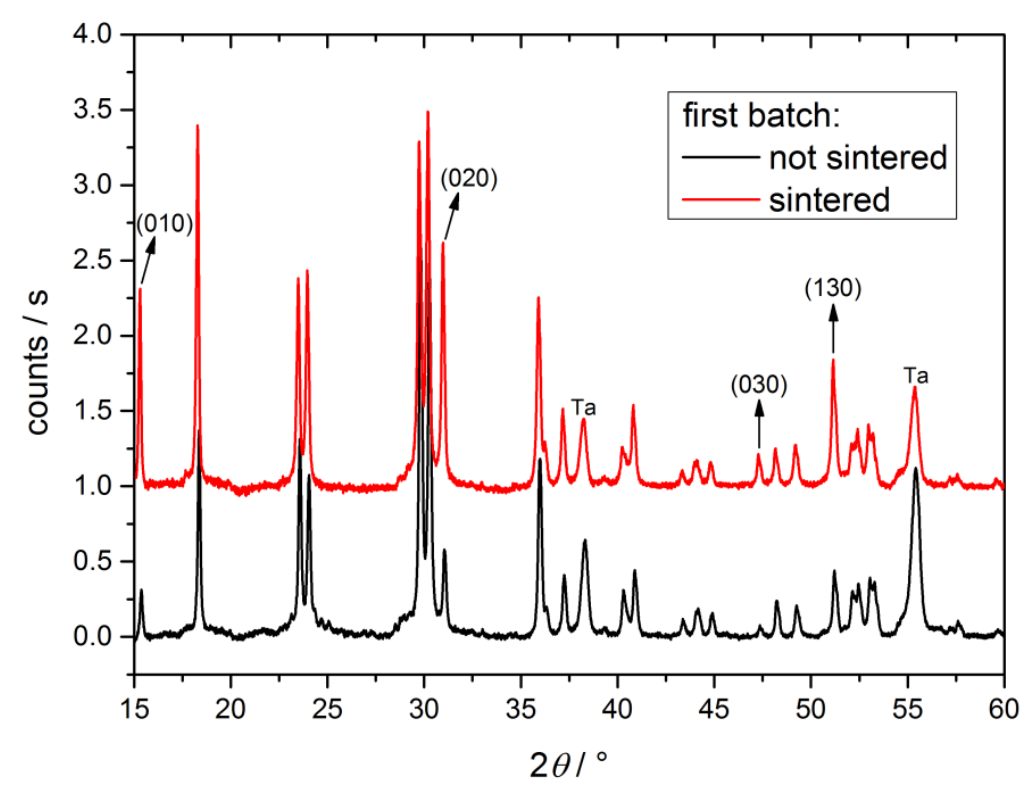

Figure 4-3: Effect of texturing on the intensity of the wolframite phase. The black line is the diffraction pattern of the nonsintered $\mathrm{MnWO}_{4}$ of the first batch while the red line represents the pattern of the sintered sample. The red line is vertically shifted for clarity. The effect is most pronounced for the labeled reflections with predominant $k$-component. Both reflections of the tantalum sample holder are labelled by "Ta". 


\subsubsection{First batch - synthesis at $800^{\circ} \mathrm{C}$}

The first batch of Mo-doped $\mathrm{MnWO}_{4}$ was synthesized according to the instruction of Meddar [18]. The reaction temperature was $800^{\circ} \mathrm{C}$ and after two cycles of synthesis, the samples were sintered at $1100^{\circ} \mathrm{C}$. In order to properly investigate the miscibility gap, samples with an amount of Mo from $x=0.0$ to $x=1.0$ were prepared. The diffraction patterns of non-sintered and sintered samples are nearly identical and therefore only patterns of sintered samples are presented in Figure 4-4. Three distinct groups can be identified: Black lines correspond to patterns of group I, which ranges from $x=0.0$ to $x=0.2$ and is characterized by the absence of the molybdate phase. In the $x=0.3$ pattern, the (220)-reflection emerges, which is the most intense Bragg-reflection of the molybdate phase and marked by an asterisk. The intensity of this peak does, however, not significantly increase with higher $x$ as expected for a conventional miscibility gap. $x=0.65$ marks the upper limit of group II, which is represented by red lines in Figure 4-4. Blue lines, on the other hand, correspond to group III. These diffraction patterns are dominated by the molybdate phase and intensity of the wolframite phase is only detectable for $x=0.7$ and $x=0.8$, which is in accordance with the upper limit of the miscibility gap of $x_{\mathrm{u}}=0.9$ found by Chang at $800^{\circ} \mathrm{C}$ [69].

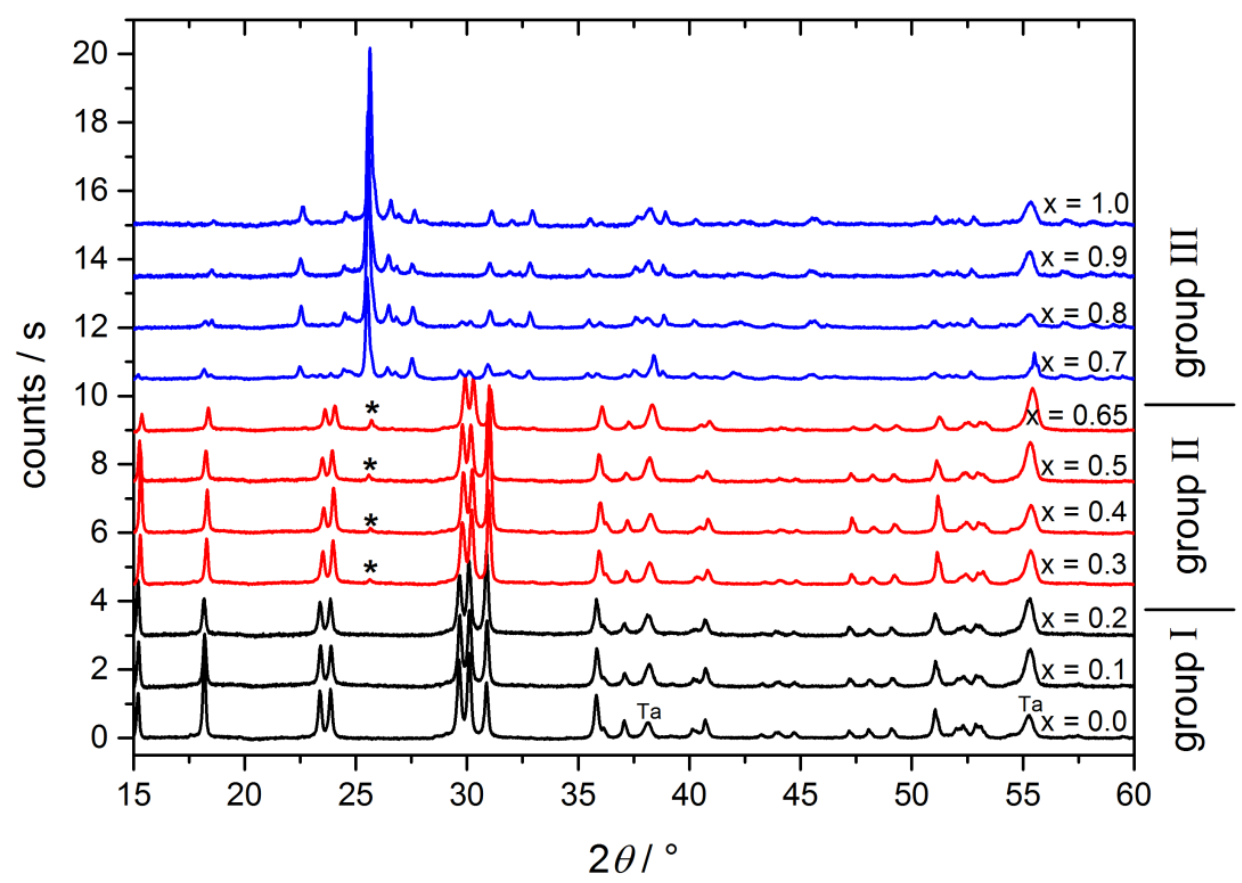

Figure 4-4: Diffraction patterns of sintered samples of the first batch. Consecutive patterns are vertically shifted for the sake of clarity. Asterisks mark the (220)-Bragg-reflection of the molybdate phase. The three colors represent different groups of diffraction patterns with distinct characteristics of the sample. See text for a detailed explanation.

Rietveld refinement was applied to determine the phase fraction and lattice parameters of the wolframite phase. The latter are $a=4.824 \pm 0.002 \AA, b=5.751 \pm 0.002 \AA, c=4.992 \pm 0.002 \AA$ and $\beta=91.15^{\circ} \pm 0.02^{\circ}$ for pure $\mathrm{MnWO}_{4}$. They are all in accordance with published data [38] with the 
exception of the monoclinic angle $\beta$, which is $0.08^{\circ}$ higher. The dependence of the lattice parameters on the amount of Mo is presented in Figure 4-5 and Figure 4-6. $a$ and $b$ are nearly constant while $c$ and $\beta$ linearly decrease for samples of group I and II. The decrease is fitted by a linear function and the fit results are presented in both figures. An orange dashed line marks the beginning of group III where the results deviate from the linear behavior.

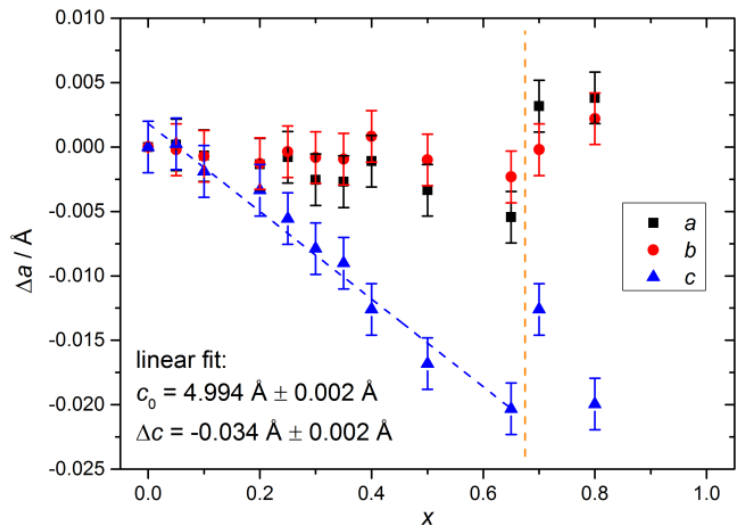

Figure 4-5: Change of the lattice parameters $a, b$ and $c$ with increased amount of Mo. The orange dashed line marks the boundary between samples of group II and III. A linear function is fitted to the data of $c$ of group I and II, which is represented by a blue dashed line.

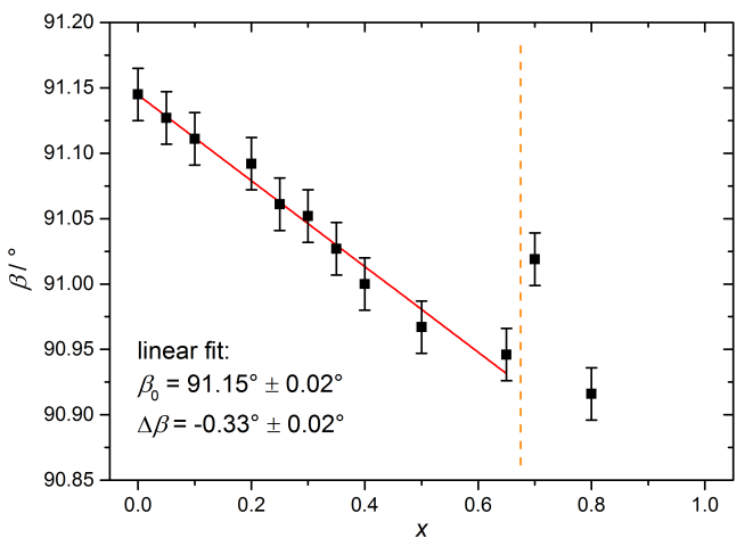

Figure 4-6: Change of the monoclinic angle $\beta$ with increased amount of Mo. The orange dashed line marks the boundary between samples of group II and III. A linear function is fitted to the data of group I and II, which is represented by a red line.

This variation of the lattice parameters results in a linear decrease of the overall unit cell volume, which is illustrated in Figure 4-7. The slope is in accordance with the decrease that was found by Meddar below $x=0.4$ although the volume of those samples is generally $0.5 \AA^{3}$ higher. This linear decrease is a clear indicator that most of the Mo atoms become part of the wolframite phase until $x=0.65$ :

$$
\mathrm{MnO}+(1-x) \mathrm{WO}_{3}+x \mathrm{MoO}_{3} \stackrel{x \leq 0.65}{\rightleftharpoons} \mathrm{MnW}_{1-x} \mathrm{Mo}_{x} \mathrm{O}_{4}
$$

For an ideal miscibility gap with the lower and upper limits of $x_{1}=0.3$ and $x_{\mathrm{u}}=0.9$, however, the unit cell volume of the wolframite phase would remain constant for $0.3<x<0.9$, as illustrated by the blue dashed line, because excess Mo would rather form molybdate phase. This would result in a linear decrease of the phase fraction $f_{W}$ of the wolframite phase, which is indicated by a blue dashed line in Figure 4-8.

$$
f_{\mathrm{w}}=\frac{x_{\mathrm{u}}-x}{x_{\mathrm{u}}-x_{1}}=\frac{0.9-x}{0.6}
$$

The reaction can be described by the equation: 
$\mathrm{MnO}+(1-x) \mathrm{WO}_{3}+x \mathrm{MoO}_{3} \stackrel{x>0.3}{\rightleftharpoons} f_{\mathrm{W}} \mathrm{MnW}_{0.7} \mathrm{Mo}_{0.3} \mathrm{O}_{4}+\left(1-f_{\mathrm{W}}\right) \mathrm{MnW}_{0.1} \mathrm{Mo}_{0.9} \mathrm{O}_{4}$

The experimental results of the phase fraction, however, suggest that no significant phase separation occurs until $x=0.65$. The molybdate phase indeed appears at $x=0.3$ and constantly increases, but still makes up less than $10 \%$ at $x=0.65$. This supports the conclusion that almost $65 \%$ of the tungsten in $\mathrm{MnWO}_{4}$ can be substituted by molybdenum. Since only the weak (220)-Bragg reflection of the molybdate is detectable, a quantitative evaluation of the phase fraction is only possible under the assumption that this reflection exhibits no pronounced texturing. Diffraction patterns of sintered and non-sintered $\mathrm{MnMoO}_{4}$ revealed that there is indeed only minor texturing of other reflections, but this can be different for a minority molybdate phase.

The present results are actually not in contradiction to Meddar, since the only evidence for a phase separation in that study is an $x=0.4$ diffraction pattern with a weak (220)-reflection of the molybdate phase, which is similar to the present $x=0.4$ pattern. Only the conclusion seems to be wrong that the emergence of this reflection marks the onset of a miscibility gap.

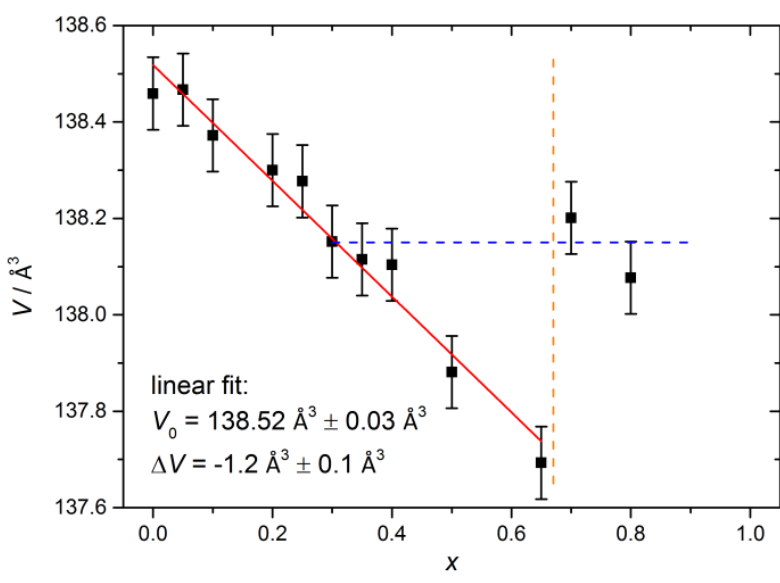

Figure 4-7: Dependence of the unit cell volume on the amount of Mo. The orange dashed line marks the boundary between samples of group II and III. The blue dashed line is the expected volume for a conventional miscibility gap with the limits $x_{1}=0.3$ and $x_{\mathrm{u}}=0.9$. A linear function is fitted to the data of group I and II, which is represented by a red line.

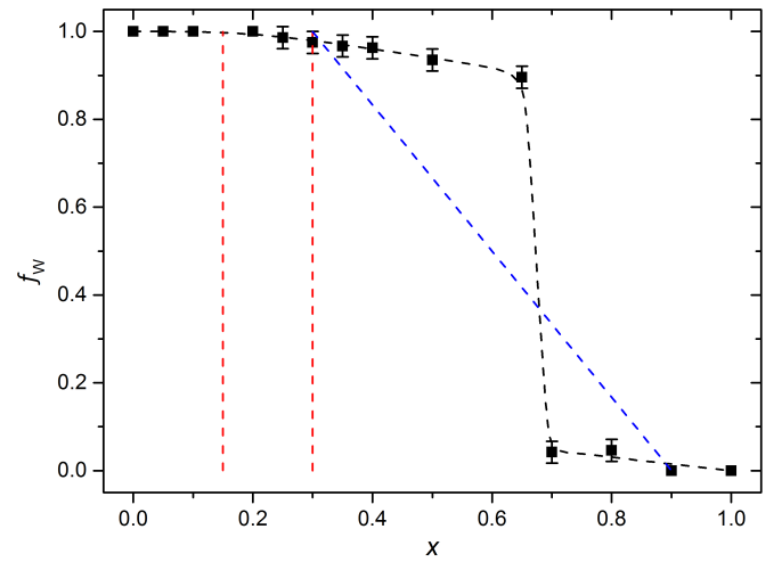

Figure 4-8: Phase fraction of the wolframite phase in dependence of the amount of Mo. The black dashed line is a guide to the eye. Red lines at $x=0.15$ and $x=0.3$ mark the lower limit of the miscibility gap as determined by Chang [69] and Meddar [18], respectively. The blue line is the theoretical phase fraction of a conventional miscibility gap with the limits $x_{1}=0.3$ and $x_{\mathrm{u}}=0.9$

Since the unit cell volume of the wolframite phase is an indicator of its Mo-content, it allows conclusions about the phase separation at $x>0.65$. The $x=0.7$ and $x=0.8$ samples contain $27 \pm 6 \%$ and $36 \pm 6 \%$ molybdenum in the wolframite phase, respectively, which is in accordance with a conventional miscibility gap. $f_{\mathrm{W}}$ of the according diffractograms, on the other hand, is significantly lower (compare to the blue dashed line in Figure 4-8) since the diffraction patterns of group III are dominated by the molybdate phase, similar like the patterns of group I and II consist mainly of 
wolframite phase. Consequently, the interpretation of the Mo-content in the wolframite phase of group III is only true under the assumption that the unit cell volume is not affected by internal stress induced by the surrounding molybdate phase, since the volume of a minority phase can inherently differ from the according pure phase.

In conclusion, most of the Mo-atoms above $x=0.65$ are part of the molybdate phase, while $30 \%$ of the tungsten in the remaining wolframite phase might still be substituted by molybdenum. This in turn implies that $27 \%$ and $17 \%$ of the Mo-atoms in the molybdate phase of the $x=0.7$ and $x=0.8$ sample, respectively, are substituted by tungsten. This is, however, not reflected by the lattice parameters of the molybdate phase which are constant within the uncertainty for all samples.

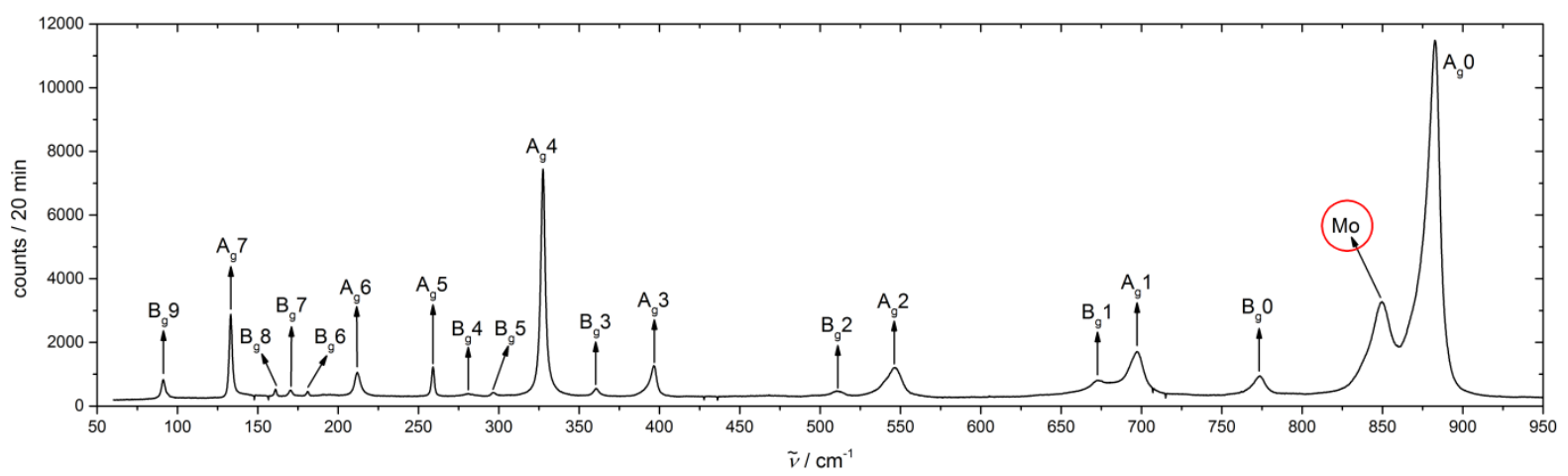

Figure 4-9: Raman spectrum of a $x=0.2$ sample of the first batch, which was sintered for two weeks. This spectrum was measured at $T=6 \mathrm{~K}$. All vibrational modes are labelled according to the Raman active irreducible representations of pure $\mathrm{MnWO}_{4}$. The only new mode is labelled as "Mo" and marked by a red circle.

Another method that yields information about the phase separation is Raman spectroscopy. The basics of this method are described in chapter 3.1.3. Figure 4-9 shows the Raman spectrum of an $x=0.2$ sample of the first batch that was sintered for two weeks. All vibrational modes are identical to that of pure $\mathrm{MnWO}_{4}$ [117-119] with only one exception: a new mode appears at $850 \mathrm{~cm}^{-1}$, further on called Mo-mode. The intensity of this mode depends on the Mo-content of the wolframite phase as illustrated in the right panel of Figure 4-10 where Raman spectra of several sintered samples of the first batch are presented. The intensity of the $A_{g} 0$-mode constantly decreases with $x$, while that of the Mo-mode increases. The $\mathrm{A}_{\mathrm{g}} \mathrm{O}$-mode is a stretching vibration of the $\mathrm{WO}_{6}$-octahedron [117] and it is therefore save to assume that the Mo-mode is a stretching vibration of the $\mathrm{MoO}_{6}$-octahedron. This supports the conclusion from Rietveld refinement that the molybdenum-content of the wolframite phase constantly increases up to $x=0.65$.

In contrast to the diffraction patterns, all Raman spectra of group II exhibit no sign of characteristic molybdate vibrations like the two modes at $930 \mathrm{~cm}^{-1}$ and $942 \mathrm{~cm}^{-1}$. The spectra of group III, on the other hand, predominantly consist of molybdate modes, which is in accordance 
with XRPD data, but the intensity relative to the wolframite modes is inconsistent. The spectrum of the $x=0.9$ sample, for example, clearly shows vibrations of the wolframite phase like the Mo-mode, although the diffraction pattern indicates the absence of any wolframite phase. Furthermore, the intensity of wolframite modes in the $x=0.8$ and $x=0.9$ spectra are higher than in the $x=0.7$ spectrum. These discrepancies of the phase fraction might be explained by the preparation of the samples. The Raman spectra were directly measured on sintered cylinders while these cylinders need to be ball milled for X-ray powder diffraction. The samples are the same in both cases but they are much more homogenous when the diffraction patterns are measured. There is a significant grain growth during sintering to such an extent that grains of the sintered cylinders are visible with bare eye, which corresponds to a size of a few $100 \mu \mathrm{m}$. This is approximately the size of the focal point of the Raman laser on the sample. Each Raman spectrum therefore might correspond only to one or a few grains and if the composition of individual grains varies, the phase fraction of the spectra is subject to local variations and might not resemble the ratio of the bulk.
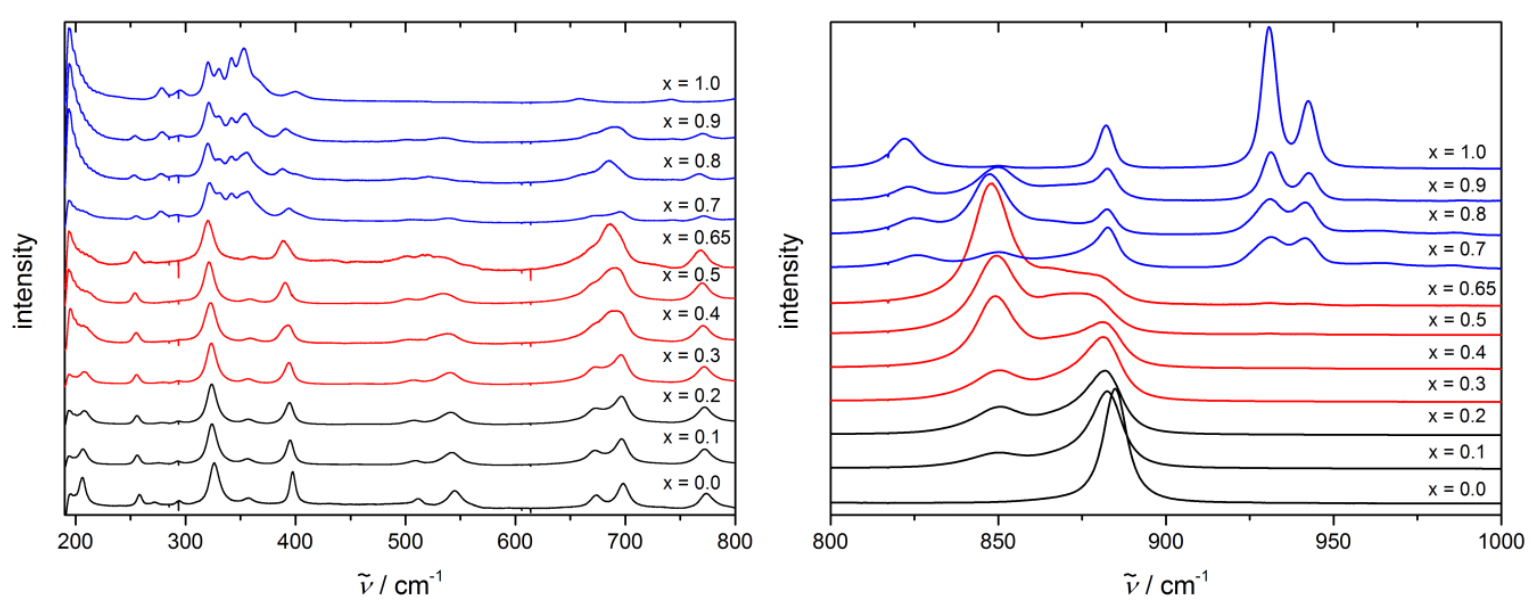

Figure 4-10: Raman spectra of sintered samples of the first batch measured at room temperature. Consecutive spectra are vertically shifted for the sake of clarity. The three colors represent the same groups with distinct characteristics of the sample as in Figure 4-4. For a detailed explanation, see text. The absolute intensities of the spectra of group I and II vary. The intensities are therefore normalized with respect to the $A_{\mathrm{g}} 4$ mode at $327 \mathrm{~cm}^{-1}$.

Consequently, a quantitative evaluation of the Raman spectra is not reasonable, but the two main conclusions from Rietveld refinement are nevertheless supported by Raman spectroscopy:

1. The Mo-content of the wolframite phase constantly increases until $x=0.65$.

2. Samples of group I and II mainly consist of wolframite phase and samples of group III mainly consist of molybdate phase. 
These conclusions are, however, in contrast to the phase diagram that was determined by Chang [69] who found that the lower and upper limit of the miscibility gap at $T=800^{\circ} \mathrm{C}$ is approximately $x_{1}=0.15$ and $x_{\mathrm{u}}=0.9$, respectively. The upper limit decreases to $x_{\mathrm{u}}=0.7$ at $1030^{\circ} \mathrm{C}$, which is irrelevant for the further considerations because the phase fraction of the present sample did not change during sintering. These contradictions might be explained by different synthesis routes. Instead of a direct synthesis from the stochiometric mixed starting materials $\mathrm{MnO}, \mathrm{WO}_{3}$ and $\mathrm{MoO}_{3}$, Chang employed a two-step synthesis where pure $\mathrm{MnWO}_{4}$ and $\mathrm{MnMoO}_{4}$ is produced first and then mixed in a stochiometric ratio for a second synthesis step. This synthesis was reproduced in the present thesis for an $x=0.5$ sample at $1100^{\circ} \mathrm{C}$, which is presented as red line in Figure $4-11$. This diffraction pattern consists of wolframite as well as molybdate phase with a phase fraction of $f_{\mathrm{W}}=0.45 \pm 5 \%$. It can be clearly distinguished from the sintered $x=0.5$ sample of the first batch, which is represented by a black line. It is rather identical to the pattern of a sample that is a mere mixture of $\mathrm{MnWO}_{4}$ and $\mathrm{MnMoO}_{4}$ with the ratio 0.5 , which corresponds to the blue line. This result is consistent with the phase diagram of Chang and it shows that the two-step synthesis yields a sample with considerable phase separation.

This furthermore suggests that either the sintered samples of the first batch or the sample after Chang's synthesis are not in thermodynamic equilibrium but rather in a kinetically hindered metastable state. Otherwise, both samples should be identical after sintering at the same temperature. Taking into consideration the odd behavior of the samples of the first batch, it seems rather likely that the wolframite phase with high Mo-content is a metastable state. This assumption is further supported by the fact that it is possible to synthesize a wolframite phase with $100 \%$ molybdenum at high pressure [120]. It remains stable at room temperature even when the pressure

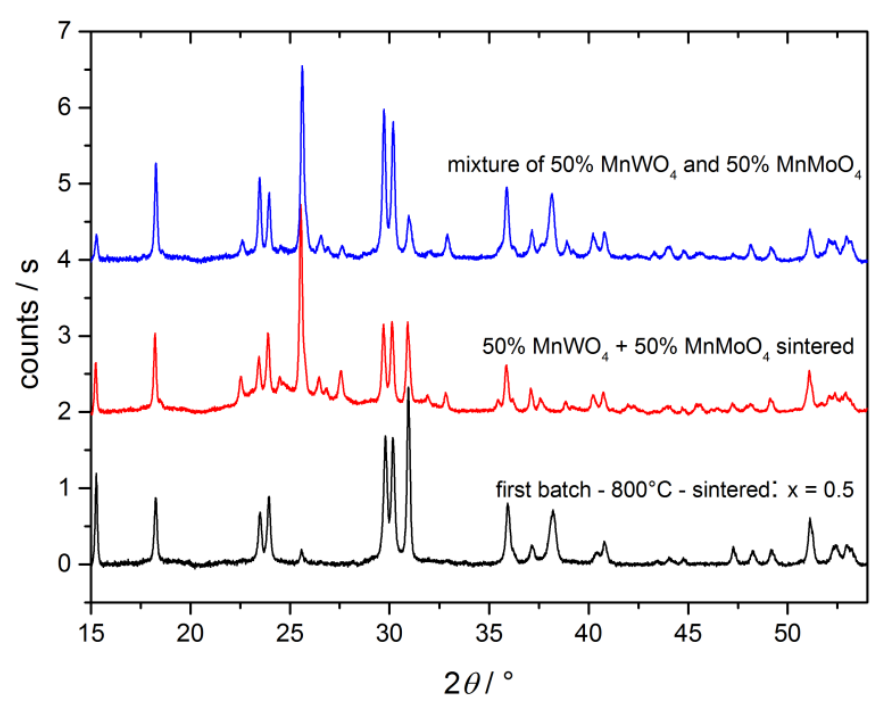

Figure 4-11: Comparison of diffraction patterns of three different samples with $x=0.5$. The black line is the sintered sample of the first batch, the red line is pure $\mathrm{MnWO}_{4}$ and $\mathrm{MnMoO}_{4}$ that was mixed and sintered at $1100^{\circ} \mathrm{C}$ and the blue line is a mere mixture of $\mathrm{MnWO}_{4}$ and $\mathrm{MnMoO}_{4}$. Red and blue lines are vertically shifted for the sake of clarity. 
is released and only transforms to the molybdate phase when heated up to $600^{\circ} \mathrm{C}$. This finding was reproduced for an $x=0.8$ sample by Blanco-Gutierrez, who could furthermore prepare a metastable wolframite phase with up to $50 \%$ molybdenum by a slightly different synthesis at $500^{\circ} \mathrm{C}$ that involves extensive ball-milling after completed reaction.

Hence, it is plausible that the synthesis at $800^{\circ} \mathrm{C}$ with monometallic oxides as starting materials leads to a similar metastable wolframite phase with up to $65 \%$ molybdenum, which remains stable during sintering, though. Low temperatures seem to be favorable for the formation of a Mo-rich wolframite phase and a synthesis below $800^{\circ} \mathrm{C}$ might therefore increase the maximum amount of Mo-atoms that can be solved in this phase. The second batch is therefore synthesized at $600^{\circ} \mathrm{C}$ and the results will be discussed in the next chapter. 


\subsubsection{Second batch - synthesis at $600^{\circ} \mathrm{C}$}

The synthesis of the second batch is similar to the first batch, but with a lower reaction temperature of $600^{\circ} \mathrm{C}$. This has consequences for many aspects of the sample preparation. The milling duration had to be increased because the diffusion is significantly reduced at lower temperatures and a smaller grain size is therefore necessary to complete the reaction. For the same reason, the reaction duration was increased and some samples even needed a third reaction cycle. After the reaction at $600^{\circ} \mathrm{C}$, some samples were sintered at $1100^{\circ} \mathrm{C}$. The diffraction patterns of the non-sintered samples are presented as red and blue lines in the left panel of Figure 4-12. The red diffractograms are similar to group II of the first batch in so far that the (220)-reflection of the molybdate phase is clearly detectable, but the intensity does not significantly increase. This changes at $x=0.7$ where the intensity of the molybdate reflections continuously increases with $x$. This in contrast to group III of the first batch, where the intensity suddenly increases from the $x=0.65$ to the $x=0.7$ diffraction pattern.
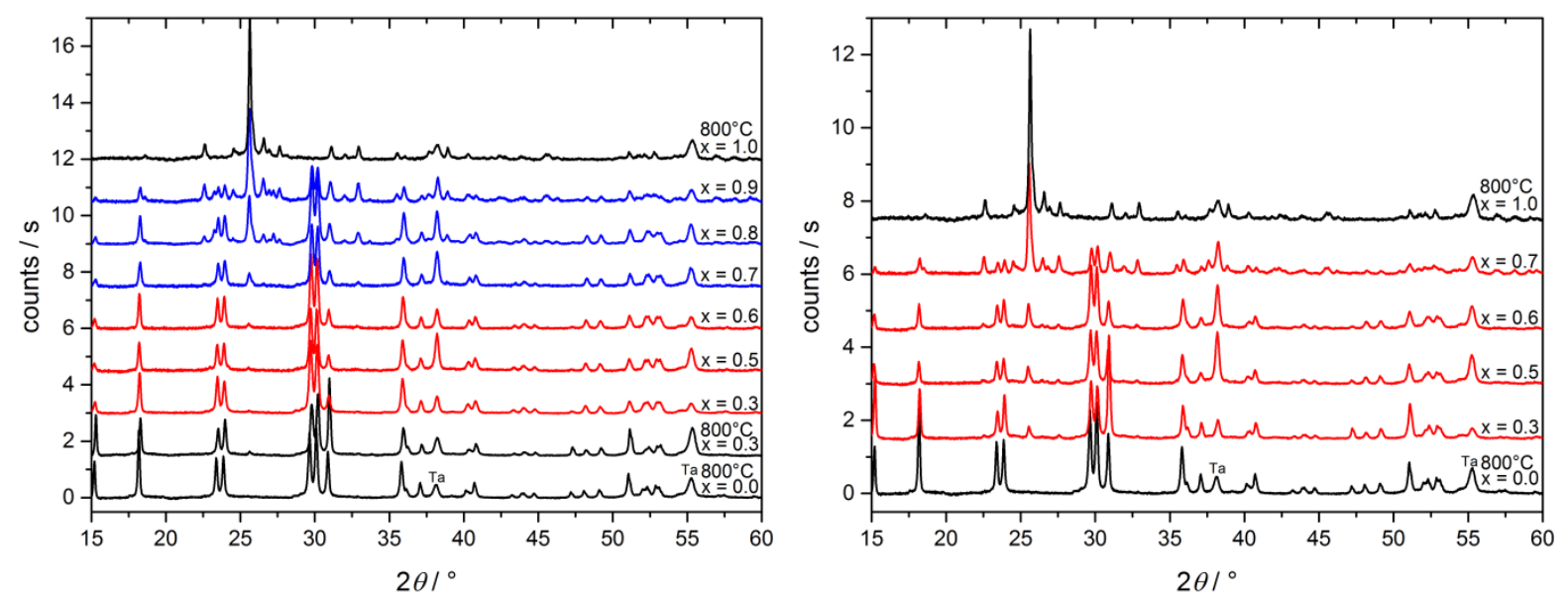

Figure 4-12: Diffraction patterns of non-sintered (left) and sintered samples (right) of the second batch. Consecutive patterns are vertically shifted for the sake of clarity. Black lines are patterns of sintered samples of the first batch for comparison. Red and blue lines in the left panel represent two groups of samples with different characteristics.

The differences between both batches are best visualized by the phase fraction of the wolframite phase $f_{\mathrm{W}}$ that is presented in Figure 4-13. Red circles represent the non-sintered samples of the second batch. The phase fraction remains at nearly $100 \%$ until $x=0.6$ and then it linearly decreases. This decrease corresponds to a conventional miscibility gap with the limits of $x_{1}=0.6$ and $x_{\mathrm{u}}=1.0$. The unit cell volume, however, which is presented as red circles in Figure 4-14, partially contradicts this interpretation because the resulting molybdenum content of the wolframite phase for the $x=0.8$ and $x=0.9$ samples would be $20 \pm 6 \%$ and $40 \pm 6 \%$, respectively, and therefore much closer to $30 \%$ (see blue dashed line), which corresponds to a miscibility gap with a lower limit of $x_{1}=0.3$. Only for the $x=0.7$ sample, the molybdenum content of $54 \pm 6 \%$ would be in accordance 
with the expected value of $60 \%$ for a gap with lower limit of $x_{1}=0.6$. However, it should be stated again that the interpretation of the Mo-content assumes that the minority wolframite phase is not affected by internal stress.

In conclusion, the results of the non-sintered $x>0.65$ samples are significantly different than the according samples of the first batch. The dependence of the phase fraction as well as of the unit cell volume on $x$ cannot be conclusively explained in terms of a conventional miscibility gap. The results of the $x \leq 0.65$ samples, on the other hand, are nearly identical to the first batch. Only $f_{\mathrm{W}}$ seems to be slightly higher. The initial expectation, however, that the maximum Mo-content of the wolframite phase can be increased further than $65 \%$ with a reduced reaction temperature is not fulfilled.

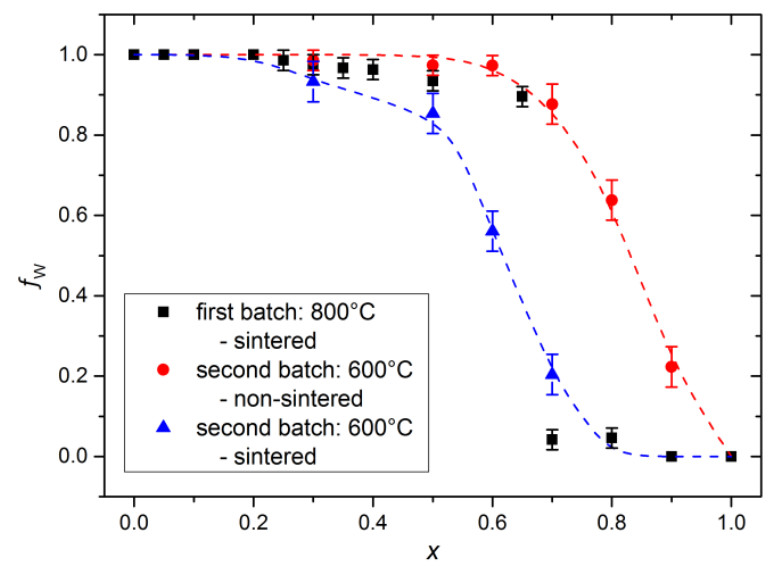

Figure 4-13: Phase fraction of the wolframite phase in dependence of the amount of Mo for different samples. Black squares are sintered samples of the first batch while red dots and blue triangles are non-sintered and sintered samples, respectively, of the second batch. Blue and red dashed lines are guides to the eye.

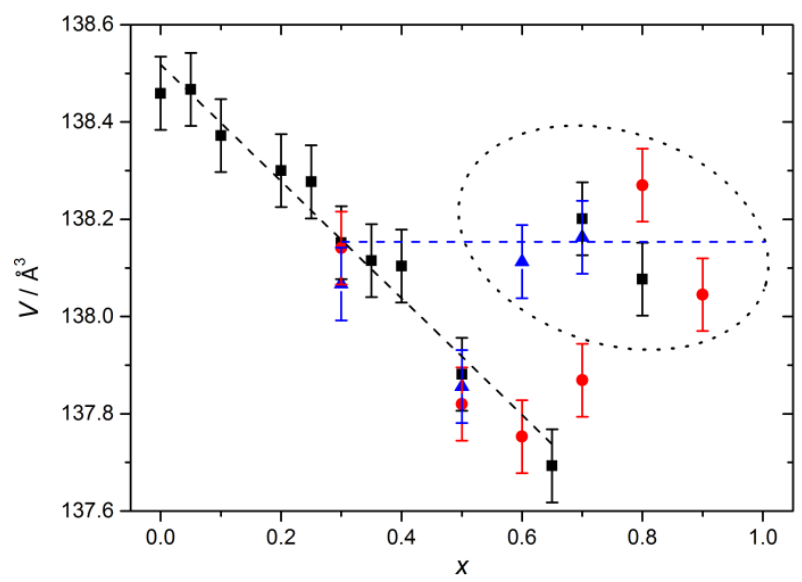

Figure 4-14: Dependence of the unit cell volume of the wolframite phase on the degree of Mo-doping for different samples. Black squares are sintered samples of the first batch while red dots and blue triangles are non-sintered and sintered samples, respectively, of the second batch. The black dashed line corresponds to the linear fit of Figure 4-7. The blue dashed line represents the according volume for $x=0.3$.

The most striking difference between the samples of the first and second batch, however, is the effect of sintering. While the diffraction patterns of the first batch were nearly identical before and after sintering, this is not the case anymore for the second batch. The diffractogram of the sintered $x=0.3$ sample (see right panel of Figure 4-12) already exhibits a stronger (220)-reflection of the molybdate phase and its intensity continuously increases with $x$. The different behavior of the sintered samples is best illustrated by the phase fraction and unit cell volume, which are presented as blue triangles in Figure 4-13 and Figure 4-14, respectively. The volume of the $x=0.3$ and $x=0.5$ samples is not affected by sintering, but the phase fraction is decreased. This means that $30 \%$ and $50 \%$ of the $\mathrm{W}$-atoms, respectively, are still substituted by Mo-atoms, but a certain amount of the wolframite phase is transformed into molybdate phase. The $x=0.6$ and $x=0.7$ samples, on the other 
hand, are now similar to the $x=0.8$ and $x=0.9$ samples before sintering. Their unit cell volume corresponds to a degree of Mo-doping of $34 \pm 6 \%$ and $30 \pm 6 \%$, which is in accordance with a miscibility gap with a lower limit of $x_{1}=0.3$. The decrease of the phase fraction, however, rather suggest a lower and upper limit of $x_{1}=0.5$ and $x_{\mathrm{u}}=0.8$. The range of phase separation seems to be shifted by $\Delta x=-0.2$ in comparison to the non-sintered samples.

There are two major conclusions from the results of the second batch:

1. The maximum amount of Mo-atoms in the wolframite phase cannot be increased by a lower reaction temperature.

2. The wolframite phase of samples with a reaction temperature of $600^{\circ} \mathrm{C}$ decomposes during sintering at $1100^{\circ} \mathrm{C}$, which is in contrast to samples that were synthesized at $800^{\circ} \mathrm{C}$. This phase separation is already observable for the $x=0.3$ sample.

The latter conclusion in fact supports the hypothesis that a wolframite phase with more than $30 \%$ molybdenum is indeed a metastable state. If the miscibility gap describes the thermodynamic equilibrium state, as assumed, it is expected that the metastable state experience a phase separation at high temperatures and that a certain amount of the wolframite phase transforms into molybdate. The question is rather why the metastable state of the $800^{\circ} \mathrm{C}$ samples does not decompose during sintering. Obviously, the variation of the reaction temperature leads to different products, but their difference does not affect any of the properties that are probed by XRPD. Measurements of the magnetic susceptibility, on the other hand, reveal that the nature of the magnetic ordering strikingly differs, which is presented and discussed in chapter 4.3.5. 


\subsubsection{Dielectric investigation}

The magnetic phase transition temperatures of $\mathrm{MnWO}_{4}$ linearly increase when tungsten atoms are replaced by up to $30 \%$ molybdenum. The interesting question is if this trend continues for samples with up to $65 \%$ Mo-content in the wolframite phase. The method of choice to probe the ferroelectric phase transition temperature $T_{\mathrm{N} 2}$ is impedance spectroscopy because a peak-like anomaly of the dielectric permittivity is expected at this temperature. Non-sintered powder of the first batch was therefore pressed to thin tablets with a diameter of $13 \mathrm{~mm}$ and a slightly varying height of around $0.5 \mathrm{~mm}$. Permittivity measurements showed, however, no sign of a phase transition even for pure $\mathrm{MnWO}_{4}$. The absence of the anomaly might be explained by the low relative density of these tablets of less than $70 \%$. Inclusions of air and grain boundaries can significantly reduce the measured capacity and the anomaly might then be too weak to be detected.

The samples were therefore sintered after pressing into tablet shape in order to increase the relative density. The sintering temperature and duration of the tablets varied depending on the Mo-content because the sintering process was started at a low temperature like $850^{\circ} \mathrm{C}$ and $T_{\text {sint }}$ was then successively increased until a relative density of approximately $80 \%$ was reached, which proved to be sufficient for impedance spectroscopy. Samples with higher $x$ were generally sintered at lower temperature but for a longer time.

Diffraction patterns of sintered tablets with a Mo-content of up to $x=0.3$ are presented in Figure 4-15. They all consist only of wolframite phase whose unit cell volume is illustrated in Figure 4-16 on the left side of the orange dashed line. The volume of most samples is significantly higher than the according volume of sintered samples of the first batch and does not follow the previously

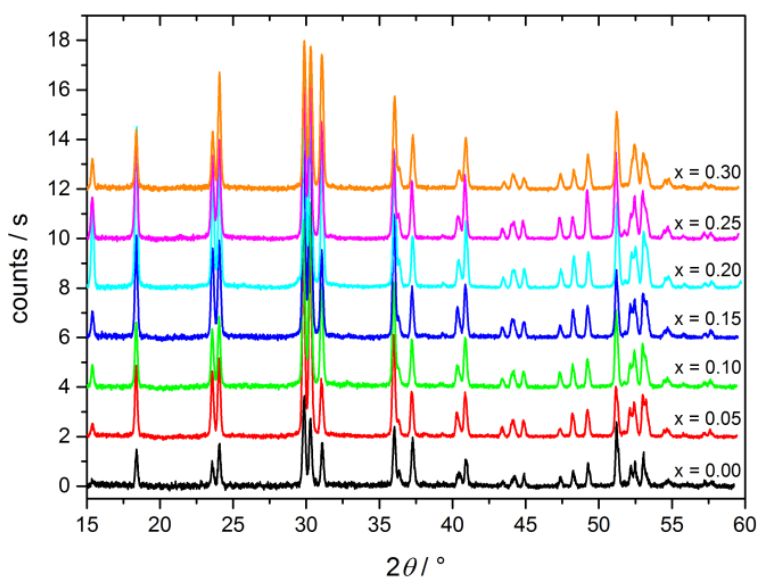

Figure 4-15: Diffraction patterns of sintered tablets. Consecutive patterns are vertically shifted for the sake of clarity. All diffraction patterns are corrected according to the $2 \theta$-offset that was determined by Rietveld-refinement.

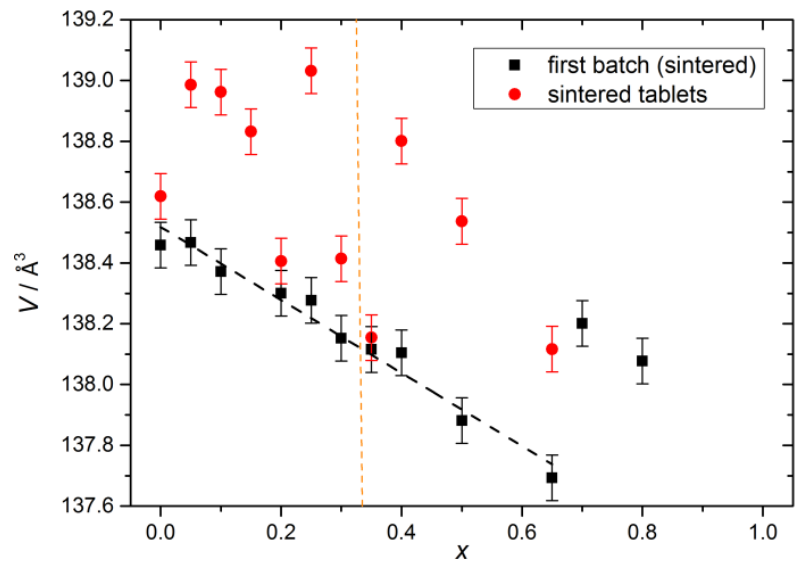

Figure 4-16: Dependence of the unit cell volume of the wolframite phase on the degree of Mo-doping for different samples. Black squares are sintered samples of the first batch and red circles are sintered tablets. The black dashed line corresponds to the linear fit of Figure 4-7. 
observed linear relation (black dashed line). The results also exhibit a higher variance between individual samples with different Mo-content. The volume change with $x$ does therefore not primarily depend on the Mo-content in the wolframite phase, but another parameter seems to be important. The sintered samples of the first batch might exhibit internal stress that can lead to a lower unit cell volume and might also be the reason for the existence of the metastable state with unusual high Mo-content. Those samples were sintered directly after the reaction while the tablets for impedance measurement underwent a further milling process before sintering, which might have released some internal stress that is build up during synthesis. The amount of remaining stress might then determine the unit cell volume.

The results of the dielectric investigation of samples with a Mo-content of up to $x=0.3$ are presented in Figure 4-17. A peak-like anomaly of the real part of the dielectric permittivity is clearly detectable for all samples. Pure $\mathrm{MnWO}_{4}$ exhibits the strongest signal while the height of the peak abruptly decreases for $x \geq 0.05$, which is in accordance with published results [18]. The maximum of the peak corresponds to the multiferroic phase transition temperature $T_{\mathrm{N} 2}$, which is determined by a least-squares fit of a Gauss-profile. The results are listed in Table 4-2 und displayed as black squares in Figure 4-18 on the left side of the orange dashed line. $T_{\mathrm{N} 2}$ linearly increases with $x$, but a slight deviation from the linear behavior appears at $x \geq 0.25$. A linear fit that includes all data with $x \leq 0.2$ yields the dependence:

$$
T_{\mathrm{N} 2}(x)=(12.7 \pm 0.1) \mathrm{K}+x \cdot(7.5 \pm 0.2) \mathrm{K}
$$

A second, less pronounced anomaly at lower temperatures emerges at $x=0.1$. Its peak center $T_{\mathrm{x}}$ is also determined by a Gaussian least-squares fit and the results are presented as red dots in Figure 4-18. $T_{\mathrm{x}}$ linearly increases with $x$, similar to $T_{\mathrm{N} 2}$, and a deviation from the linear behavior is also

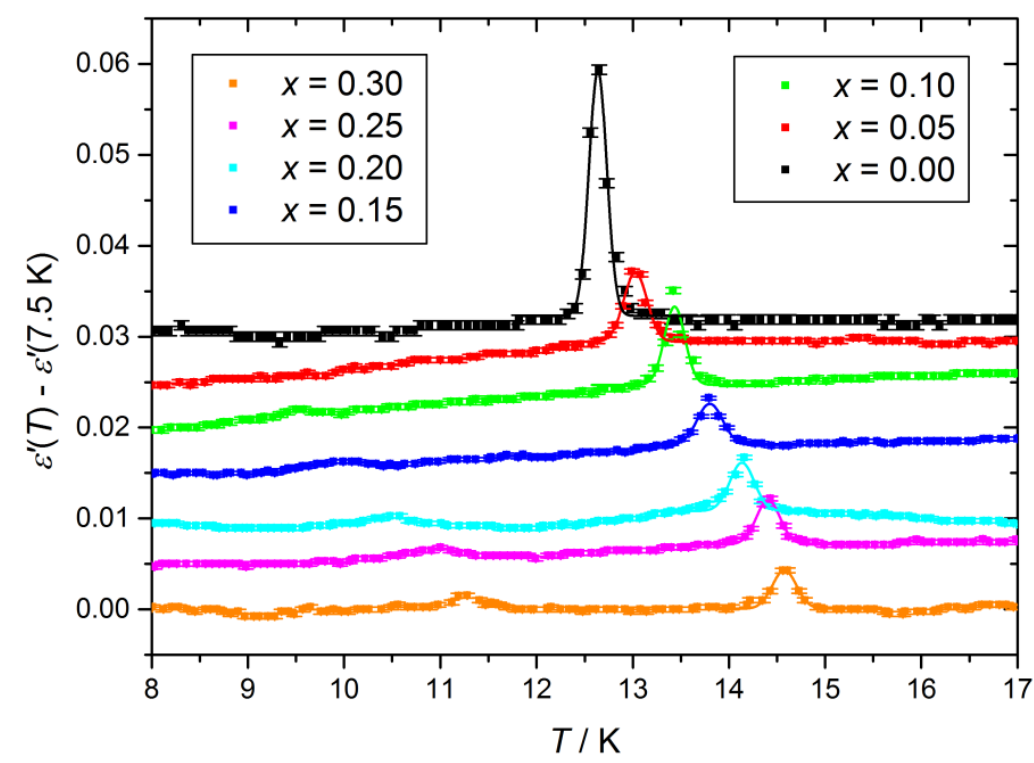

Figure 4-17: Real part of the dielectric permittivity of sintered tablets. The frequency of the AC voltage $U_{0}$ is $550 \mathrm{kHz}$. Consecutive plots are vertically shifted for the sake of clarity. Solid lines are Gauss-profiles of the peak-like anomaly at $T_{\mathrm{N} 2}$ that are determined by a leastsquares fit. 
observed for the $x=0.3$ sample. The slope of the $x \leq 0.25$ samples is $\Delta T_{\mathrm{x}}=9.5 \pm 0.5 \mathrm{~K}$ and therefore higher than $T_{\mathrm{N} 2}$.

\begin{tabular}{|c|c|c|}
\hline$x$ & $T_{\mathrm{N} 2}$ & $T_{\mathrm{x}}$ \\
\hline 0.00 & $12.6 \pm 0.1$ & - \\
\hline 0.05 & $13.0 \pm 0.1$ & - \\
\hline 0.10 & $13.4 \pm 0.1$ & $9.5 \pm 0.1$ \\
\hline 0.15 & $13.8 \pm 0.1$ & $9.9 \pm 0.1$ \\
\hline 0.20 & $14.1 \pm 0.1$ & $10.8 \pm 0.1$ \\
\hline 0.25 & $14.4 \pm 0.1$ & $10.9 \pm 0.1$ \\
\hline 0.30 & $14.6 \pm 0.1$ & $11.2 \pm 0.1$ \\
\hline 0.35 & $14.7 \pm 0.1$ & $11.5 \pm 0.1$ \\
\hline 0.40 & $14.7 \pm 0.1$ & $11.6 \pm 0.1$ \\
\hline 0.50 & $14.8 \pm 0.1$ & $11.6 \pm 0.1$ \\
\hline
\end{tabular}

Table 4-2: Dependence of both anomalies of the dielectric permittivity on the amount of Mo. $T_{\mathrm{N} 2}$ ist the multiferroic phase transition temperature and $T_{\mathrm{x}}$ represents the temperature of the anomaly with unknown origin.

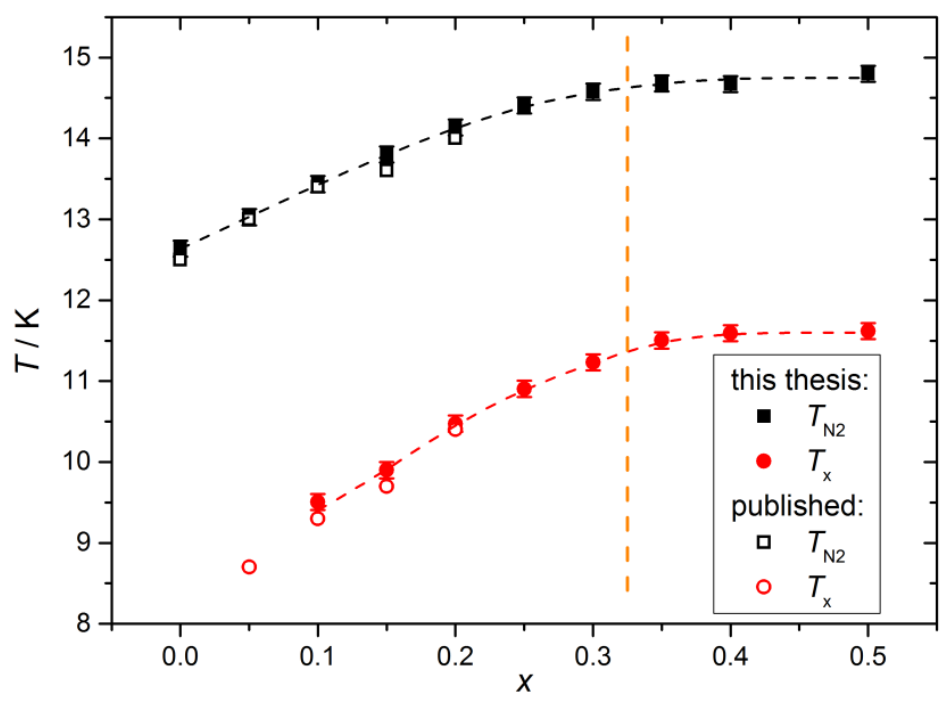

Figure 4-18: Dependence of both anomalies of the dielectric permittivity on the amount of Mo. Black squares correspond to the phase transition temperature $T_{\mathrm{N} 2}$ and red circles represent the temperature $T_{\mathrm{x}}$ of the anomaly with unknown origin. Filled symbols are results of this thesis while open symbols are published results [63]. Black and red dashed lines are guides to the eye. The orange dashed line marks the emergence of the molybdate phase.

The (220)-reflection of the molybdate phase first appears in the diffraction pattern of the $x=0.35$ sample, which is presented in Figure $4-19$. The intensity of this reflection significantly increases with $x$ and the phase fraction of the wolframite phase consequently decreases, which is depicted as red dots in Figure 4-20. This decrease in fact corresponds to the phase separation of a conventional miscibility gap with the lower and upper limit of $x_{1}=0.35$ and $x_{\mathrm{u}}=0.9$, respectively, as indicated by a blue dashed line. The Mo-content of the wolframite phase should therefore not exceed $35 \%$. The unit cell volume of the wolframite phase is presented in Figure 4-16 on the right side of the orange dashed line. Like for the $x \leq 0.3$ samples, the volume is significantly higher than that of the sintered samples of the first batch, except for the $x=0.35$ sample. It is therefore not reasonable to draw conclusions about the Mo-content of the wolframite phase from its unit cell volume. 


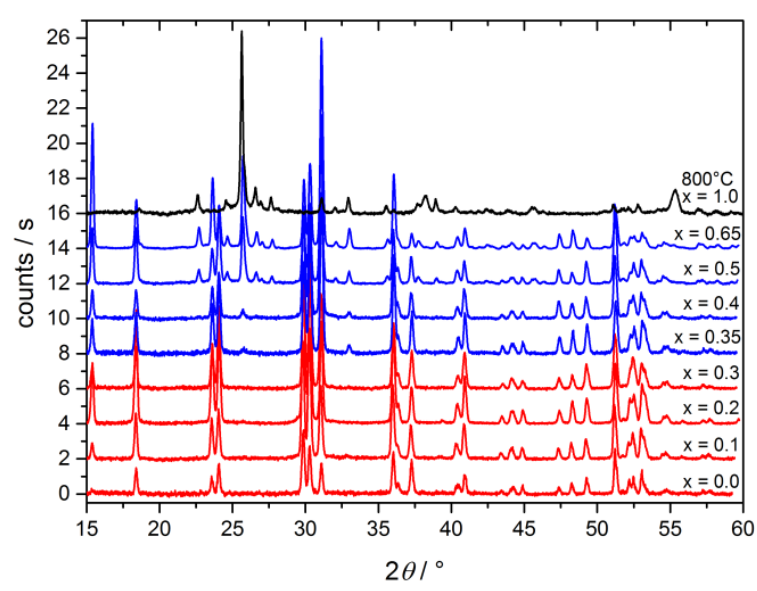

Figure 4-19: Diffraction patterns of sintered tablets. Consecutive patterns are vertically shifted for the sake of clarity. The black line is the pattern of a sintered sample of the first batch for comparison. Red and blue lines represent two groups of samples with different characteristics. All diffraction patterns are corrected according to the $2 \theta$-offset that was determined by Rietveldrefinement.

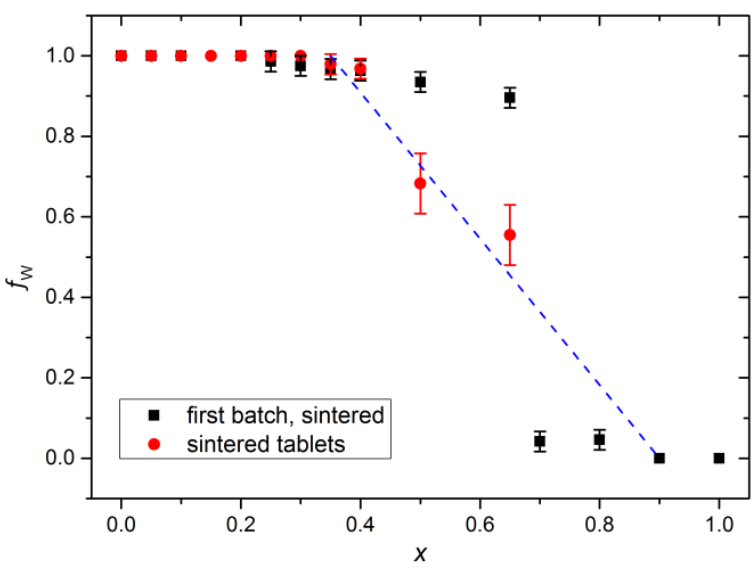

Figure 4-20: Dependence of the phase fraction of the wolframite phase on the total amount of Mo for different samples. Black squares are sintered samples of the first batch while red dots are sintered tablets. The blue line is the phase fraction of a conventional miscibility gap with the boundaries $x_{\mathrm{I}}=0.35$ and $x_{\mathrm{u}}=0.9$.

The assumption that the Mo-content of the wolframite phase does not exceed $35 \%$ is, on the other hand, supported by permittivity measurements, which are depicted in Figure 4-21. Both anomalies of the real part appear at the same temperature for all samples with $0.35 \leq x \leq 0.5$, while the $x=0.65$ sample does not exhibit any anomaly at all. The resulting values of $T_{\mathrm{N} 2}$ and $T_{\mathrm{x}}$ are presented in Figure 4-18 on the right side of the orange dashed line. They are constant within the uncertainty for all three samples, which is in accordance with $35 \%$ Mo in the wolframite phase. Only the absence of the signal in the $x=0.65$ sample cannot be explained since the anomalies of the remaining wolframite phase are still expected to be detectable.

The present results from X-ray diffraction as well as dielectric measurements are in accordance

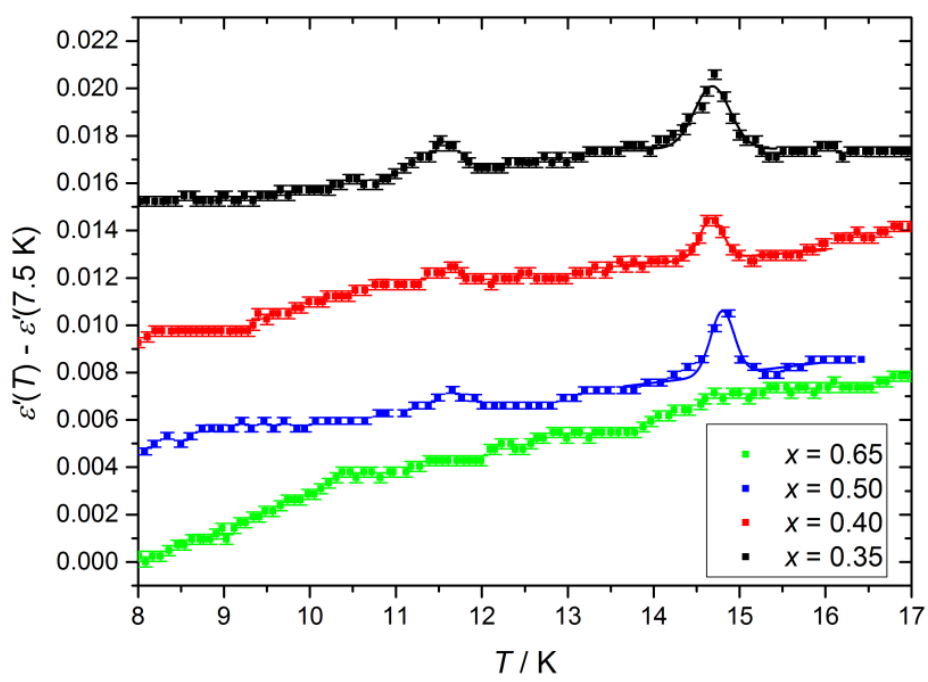

Figure 4-21: Real part of the dielectric permittivity for different sintered tablets. The frequency of the $A C$ voltage $U_{0}$ is $550 \mathrm{kHz}$. Consecutive plots are vertically shifted for the sake of clarity. Solid lines are Gauss-profiles of the peak-like anomaly at $T_{\mathrm{N} 2}$ that are determined by a least-squares fit. 
with a conventional miscibility gap with the limits $x_{1}=0.35$ and $x_{\mathrm{u}}=0.9$. Consequently, the metastable state of the non-sintered samples is decomposed during sintering and the tablets are finally in thermodynamic equilibrium. This is, however, in contrast to the sintered samples of the first batch, which still remained in the metastable state even after sintering for two days at $1100^{\circ} \mathrm{C}$. This is especially peculiar because most tablets were sintered at significantly lower temperatures than $1100^{\circ} \mathrm{C}$, which decreases the likelihood for decomposition due to a decreased diffusion of atoms.

The sintered samples of the second batch also exhibit a pronounced phase separation, which may be due to the different reaction temperatures of the first and second batch. This can, however, not be an explanation for the sintered tablets, because non-sintered powder of the first batch was used. There are two major differences between the sintered samples of the first batch and the sintered tablets. Firstly, the shape of the samples differs. The thin tablets have a significantly higher surface-to-bulk-ratio than the cylinders of the first batch. If the surface decomposes easier than the bulk, this might explain the different behavior. Secondly, as mentioned above, the cylinders of the first batch were directly sintered after completed reaction. For the dielectric measurements on the other hand, the cylinders had to be ball milled before the resulting powder could be pressed into tablet shape. The milling process decreases the grain size of the non-sintered material and can release internal stress that might have built up during the reaction and stabilized the metastable state with unusual high Mo-content. Both effects could have an impact on the phase separation during sintering.

Therefore, another approach was tested to produce sintered tablets, which avoids both aforementioned problems. Sintered cylinders with Mo-content of $x=0.40,0.45$ and 0.50 were prepared exactly like the according samples of the first batch and then cut into thin slices with a diamond wire saw. Diffraction patterns of these samples before sintering are presented as black lines in Figure 4-22. All three diffractograms exhibit a weak (220)-reflection of the molybdate phase, which is in accordance with the samples of the first batch. Red lines in Figure 4-22 represent the diffraction patterns of the sintered and cut tablets, which differ significantly. The intensity of the molybdate phase is considerably increased, which is reflected by the phase fraction of the wolframite phase that is presented in Table 4-3. These results suggest that a phase separation occurred during sintering, which is further supported by measurement of the dielectric permittivity (see Figure 4-23). The anomalies of the real part correspond to a Mo-content of $30 \%$ in the wolframite phase for all three samples. 


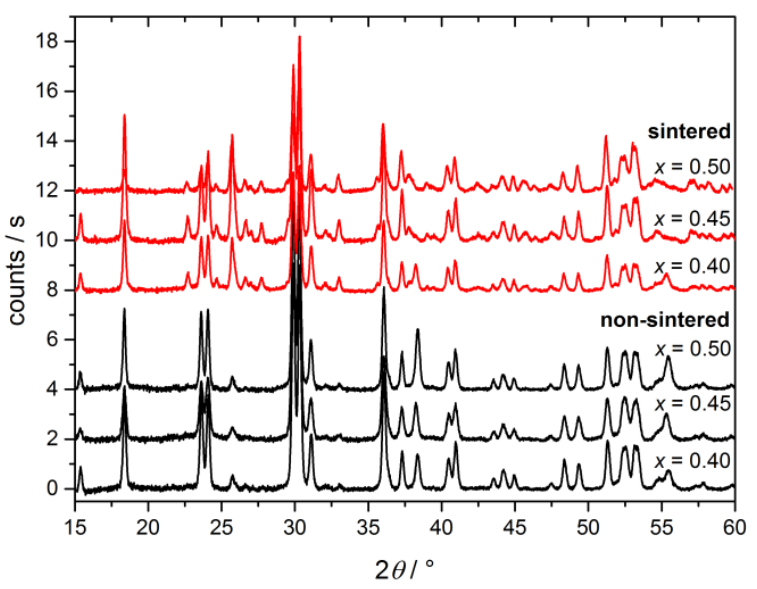

Figure 4-22: Diffraction patterns of non-sintered (black lines) and sintered samples (red lines) that were synthesized like the first batch. Consecutive patterns are vertically shifted for the sake of clarity.

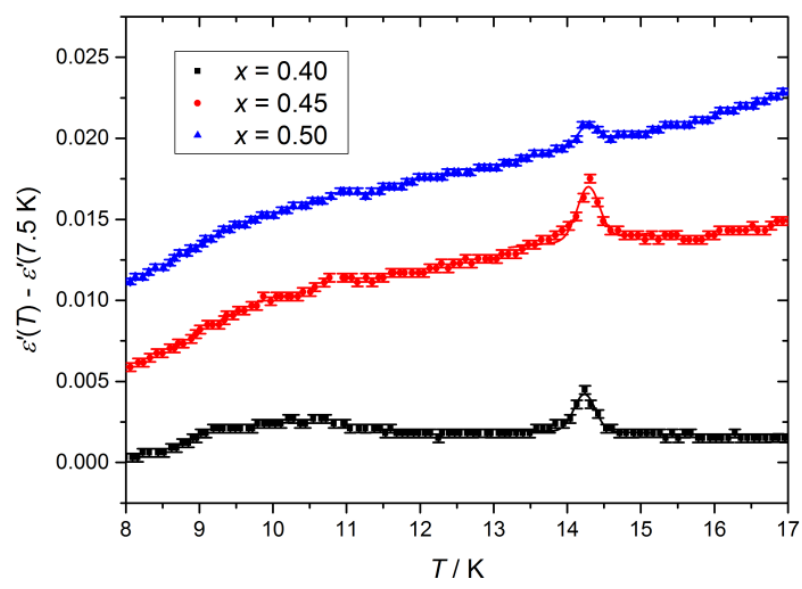

Figure 4-23: Real part of the dielectric permittivity of different sintered samples that were synthesized like the first batch. The frequency of the AC voltage $U_{0}$ is $550 \mathrm{kHz}$. Consecutive plots are vertically shifted for the sake of clarity. Solid lines are Gaussprofiles of the peak-like anomaly at $T_{\mathrm{N} 2}$ that are determined by a least-squares fit.

Table 4-3: Phase fraction of the wolframite phase for non-sintered and sintered samples that were synthesized like the first batch.

\begin{tabular}{|c|c|c|c|}
\hline$f_{\mathrm{W}}$ & $x=0.40$ & $x=0.45$ & $x=0.50$ \\
\hline non-sintered & $95 \pm 3 \%$ & $95 \pm 3 \%$ & $96 \pm 3 \%$ \\
\hline sintered & $65 \pm 8 \%$ & $69 \pm 8 \%$ & $77 \pm 8 \%$ \\
\hline
\end{tabular}

These findings are in direct contrast to the results of the first batch, since the samples were prepared in the same way, but the effect of the sintering process is different. All parameters like the duration of milling, temperature and duration of the reaction, number of reaction cycles, temperature and duration of the sintering process are the same. The sample cylinders were also sintered directly after the synthesis without intermediary milling. Consequently, there must be a further parameter that determines the stability of the metastable state and that has been overlooked so far. This unknown parameter might also be the reason why the samples of the second batch decompose during sintering rather than the lower reaction temperature.

Blanco-Gutierrez claims that it is the extensive ball milling after the actual reaction that induces internal stress in the samples and thereby produces the metastable wolframite phase with up to $50 \%$ molybdenum, which then decomposes at higher temperatures [71]. In fact, the metastable samples of the first and second batch were ball-milled for a few minutes before the XRPD measurements, while all decomposed tablets were directly measured as tablet without an additional milling process. However, the samples of the first and second batch were all milled under similar conditions, but their properties differ widely. Furthermore, the sintered cylinders of the first batch, 
which were examined by Raman spectroscopy, clearly exhibit the metastable wolframite phase with $65 \%$ molybdenum, although these samples were not milled before the measurement. Ball-milling can therefore not be the only explanation for the occurrence of the metastable wolframite phase, but it might play an important role.

Nevertheless, it is actually hard to reproduce the exact conditions for each milling process. The number and size of the balls, the rotation speed of the milling beaker and the duration of the milling process are easy to control, but also the amount of the sample and its grain size are relevant for the milling result and can vary between individual samples. Furthermore, the balls are subject of wear that increases the roughness of their surface and can also affect the force that the balls can transfer onto the sample. Consequently, these uncertainties might be the unknown parameter that determines the stability of individual samples. However, there are some more properties of the synthesis that might not be defined well enough. The pressure that was put onto the powder when it was pressed into cylinder shape might vary and so does the height of the cylinders, which in turn determines the surface to bulk ratio. This might also affect how much internal stress builds up during the reaction. Furthermore, the heating and cooling rates of the muffle furnace at the beginning and end of the reaction and of the sintering process were not directly controlled. Especially the cooling rate can have an important influence on the phase separation of a sample.

This unfortunately leaves the conclusion that the synthesis of the first batch is currently not reproducible and it was not possible to determine the multiferroic phase transition temperature $T_{\mathrm{N} 2}$ of samples with a Mo-content of more than $30 \%$ in the wolframite phase by permittivity measurements. Another method to obtain magnetic phase transition temperatures is the measurement of the magnetic susceptibility, which will be discussed in the next chapter. 


\subsubsection{Magnetic susceptibility of selected samples}

The measurement of the magnetic susceptibility is another way to determine whether the magnetic phase transition temperatures of the wolframite phase continuously increase even for a Mo-content over $30 \%$. The advantage of this method compared to impedance spectroscopy is that the samples are measured as powder and the results can be directly related to the according diffraction patterns. Therefore, no sintering is necessary, which would otherwise alter the properties of the samples and might induce a phase separation.

For these experiments, a SQUID magnetometer was used which allows the measurement of the magnetization while an external field is applied. If the sample exhibits no spontaneous magnetization $M_{(s)}$, the measured magnetization is directly proportional to the magnetic susceptibility $\chi$ (see also chapter 2.1.2):

$$
M=\chi \cdot H
$$

Figure 4-24 and Figure 4-25 provide an overview over the magnetization of several sintered samples of the first batch and non-sintered samples of the second batch, respectively, in a temperature range from room-temperature to $2 \mathrm{~K}$. The magnetization increases for all samples on cooling according to the Curie-Weiß-law until $50 \mathrm{~K}$. At lower temperatures, there are several samplespecific anomalies and the magnetization finally decreases with the onset of antiferromagnetic order. The maximum magnetization is therefore an indication for the phase transition temperature $T_{\mathrm{N} 3}$ of the wolframite phase.

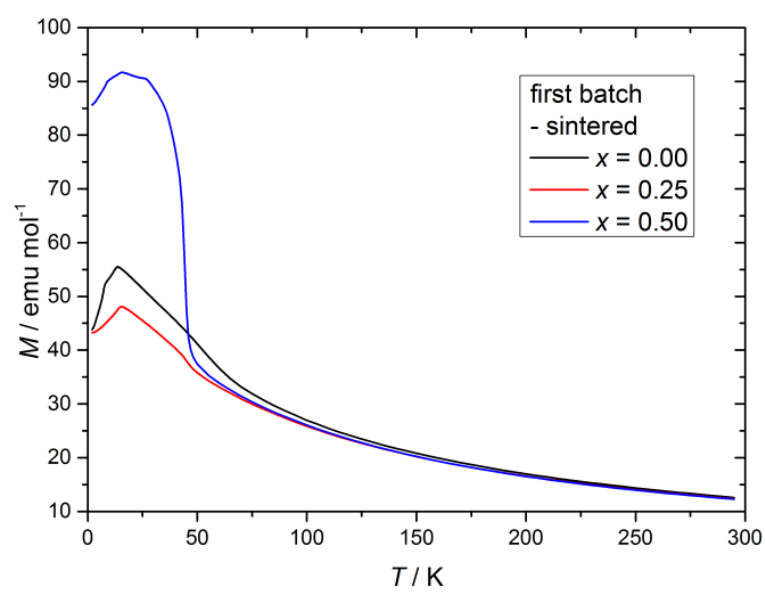

Figure 4-24: Temperature dependence of the magnetization of several sintered samples of the first batch for an external field of $H=1000$ Oe.

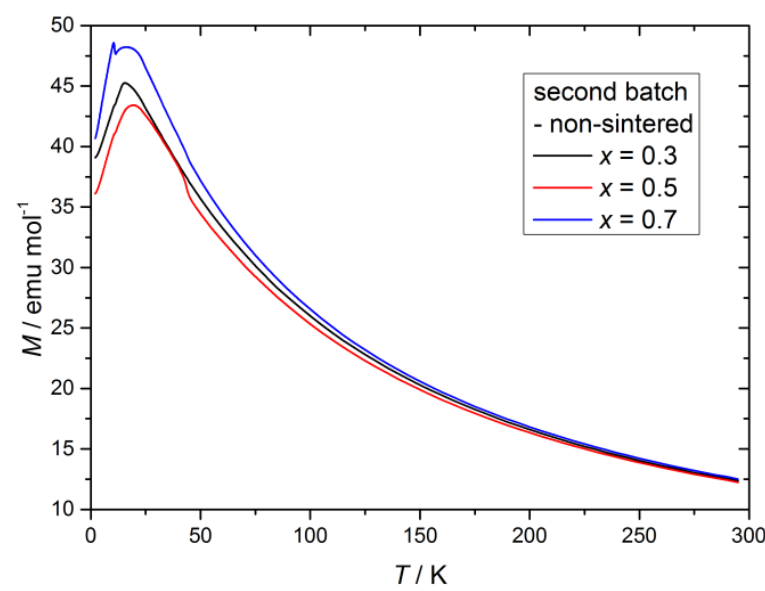

Figure 4-25: Temperature dependence of the magnetization of several non-sintered samples of the second batch for an external field of $H=1000$ Oe.

The low-temperature magnetization of pure $\mathrm{MnWO}_{4}$ of the first batch is compared to the $x=0.25$ sample of the same batch and the $x=0.30$ sample of the second batch in Figure 4-26, while 
the according temperature derivatives are presented in Figure 4-27. The maximum magnetization of $\mathrm{MnWO}_{4}$ and therefore the zero of the derivative correspond to the transition temperature $T_{\mathrm{N} 3}=13.7 \pm 0.1 \mathrm{~K}$. The lock-in phase transition at $T_{\mathrm{N} 1}=7.8 \pm 0.5 \mathrm{~K}$, on the other hand, is characterized by a sudden change of slope of the magnetization. Both temperatures are in agreement with published data [38]. The anomaly at $T_{\mathrm{N} 1}$ is completely absent for the $x=0.25$ and $x=0.3$ sample and therefore no commensurate phase is present. Only the transition at $T_{\mathrm{N} 3}$ is observed, which occurs at a higher temperature of $T_{\mathrm{N} 3}=15.4 \pm 0.1 \mathrm{~K}$ for both samples. This transition is broader and less defined for the non-sintered sample, which might indicate that not all crystallites have the same Mo-content. Consequently, $T_{\mathrm{N} 3}$ varies slightly for different grains of the sample and the slope of the magnetization changes less abruptly. The $x=0.25$ sample, on the other hand, was synthesized at a higher temperature and sintered afterwards, which results in an increased diffusion and might therefore lead to a higher homogeneity of the sample and a sharper transition. Furthermore, this sample exhibits a step-like anomaly of the magnetization at $T \approx 45 \mathrm{~K}$, which manifests in a minimum of the first derivative that is not observed for both other samples.

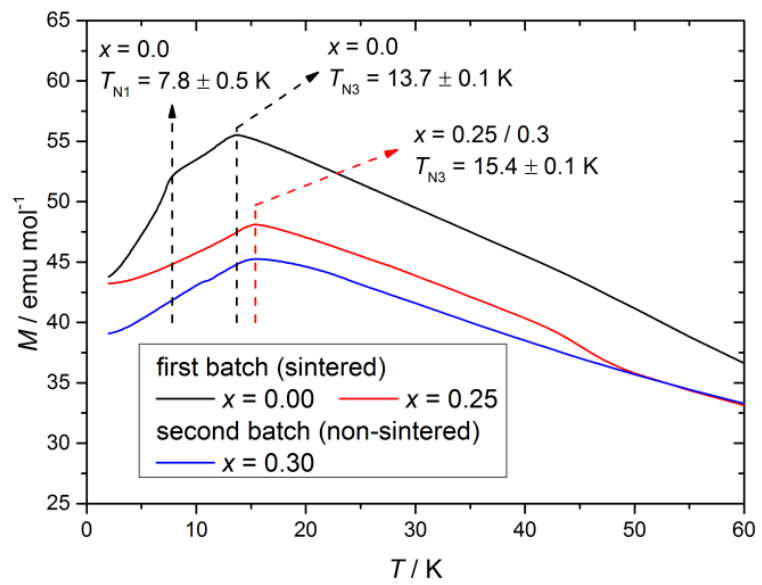

Figure 4-26: Temperature dependence of the magnetization of two sintered samples of the first batch and a non-sintered sample of the second batch for an external field of $H=1000$ Oe. Several phase transition temperatures are marked by black and red dashed lines.

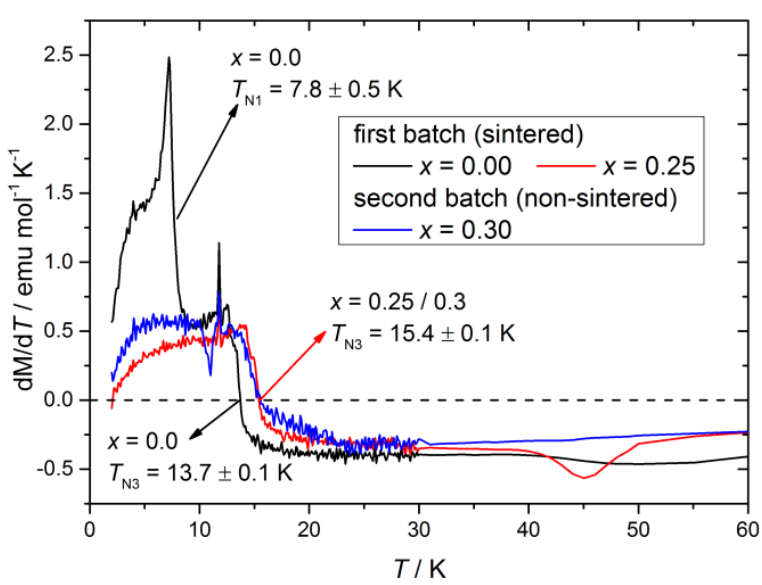

Figure 4-27: Temperature derivative of the the magnetization of two sintered sample of the first batch and a non-sintered sample of the second batch for an external field of $H=1000$ Oe.

The magnetization of the $x=0.3$ sample is compared to the $x=0.5$ and $x=0.7$ samples of the same batch in Figure 4-28, while the according derivatives are presented in Figure 4-29. All three samples exhibit a distinct anomaly of the magnetization at $T=10.4 \pm 0.1 \mathrm{~K}$, which is close to the Néeltemperature of $\mathrm{MnMoO}_{4}$ at $T_{\mathrm{N}}=9.8 \pm 0.1 \mathrm{~K}$ [121] and can therefore be attributed to the antiferromagnetic phase transition of the molybdate phase. This is supported by the fact that the anomaly is less pronounced for the $x=0.3$ and $x=0.5$ samples, which have a phase fraction of the molybdate phase of only $f_{M}=1 \pm 3 \%$ and $f_{M}=3 \pm 3 \%$, respectively, while the $x=0.7$ sample exhibits a distinct maximum of the magnetization and $f_{\mathrm{M}}=12 \pm 5 \%$ is also significantly higher. Furthermore, 
the $x=0.0$ and $x=0.25$ samples of the first batch lack this anomaly and also show no sign of molybdate phase in the according diffraction patterns (see Figure 4-26 and Figure 4-4).

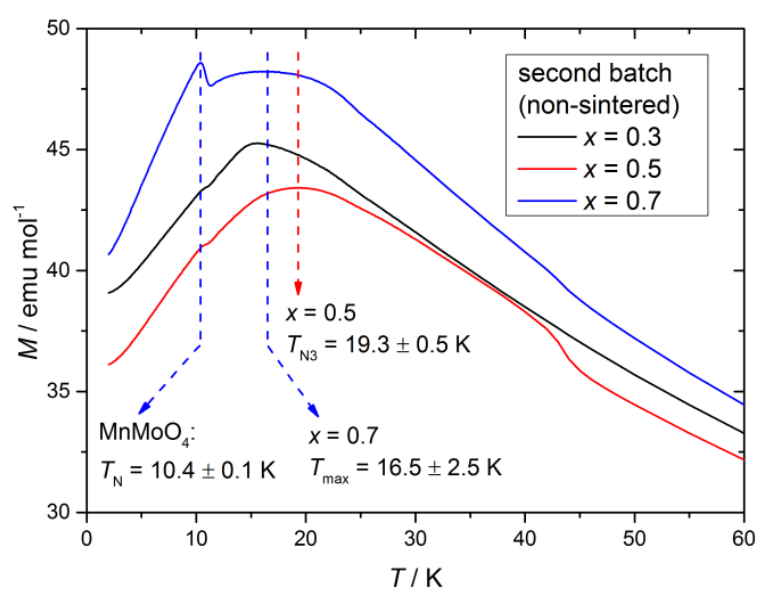

Figure 4-28: Temperature dependence of the magnetization of several non-sintered samples of the second batch for an external field of $H=1000$ Oe. Several relevant temperatures are marked by red and blue dashed lines.

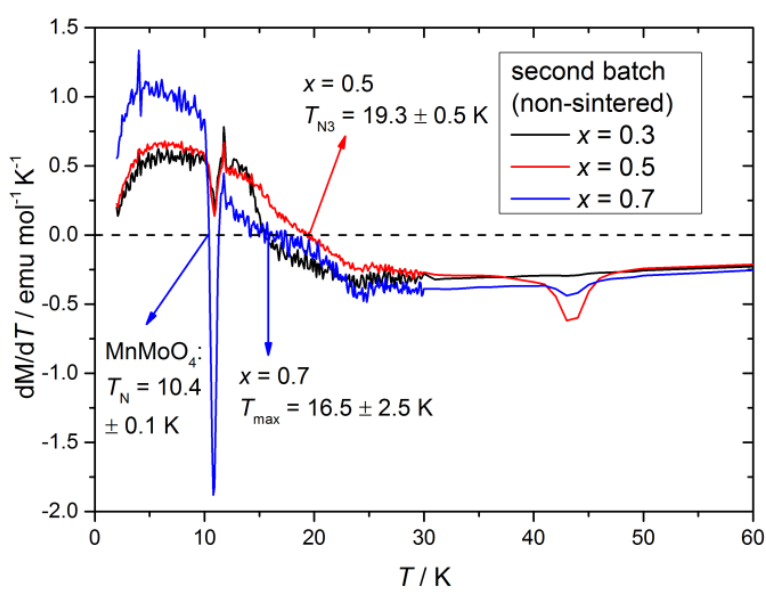

Figure 4-29: Temperature derivative of magnetization of several non-sintered samples of the second batch for an external field of $H=1000$ Oe.

The maximum magnetization of the $x=0.5$ sample appears at a temperature of $T_{\mathrm{N} 3}=19.3 \pm 0.5 \mathrm{~K}$, which strongly deviates from the linear relation of the $x \leq 0.3$ samples by more than $2 \mathrm{~K}$ as depicted in Figure 4-30. This might be an indication that the magnetic phase transition temperatures increase even stronger when more than $30 \%$ Mo is solved in the wolframite phase. This transition is, however, broader than in the case of the $x=0.3$ sample and stretches over a temperature range of approximately $10 \mathrm{~K}$, which suggests a further increased inhomogeneity of this sample.

The magnetization of the $x=0.7$ sample, however, exhibits its maximum at a lower temperature of $T_{\max }=16.5 \pm 2.5 \mathrm{~K}$. This maximum is accompanied by a plateau, which is even broader than for the $x=0.5$ sample. This is again a sign for an enhanced inhomogeneity, but another problem arises for this sample. The measured magnetization is an overlap of signals of the wolframite as well as molybdate phase. Therefore, $T_{\max }$ cannot be identified as $T_{\mathrm{N} 3}$ which renders the determination of the antiferromagnetic phase transition temperature of the wolframite phase impossible for this sample.

The step-like anomaly at $\mathrm{T} \approx 45 \mathrm{~K}$, which is also observed for the sintered $x=0.25$ sample of the first batch and which is absent in the non-sintered $x=0.3$ sample of the second batch, can be found in the non-sintered $x=0.5$ and $x=0.7$ samples of the second batch. Consequently, the anomaly cannot be a result of the sintering process. Since the compositions of the $x=0.25$ and 
$x=0.3$ samples are quite similar, the amount of Mo in the wolframite phase can also be ruled out as origin of the anomaly.

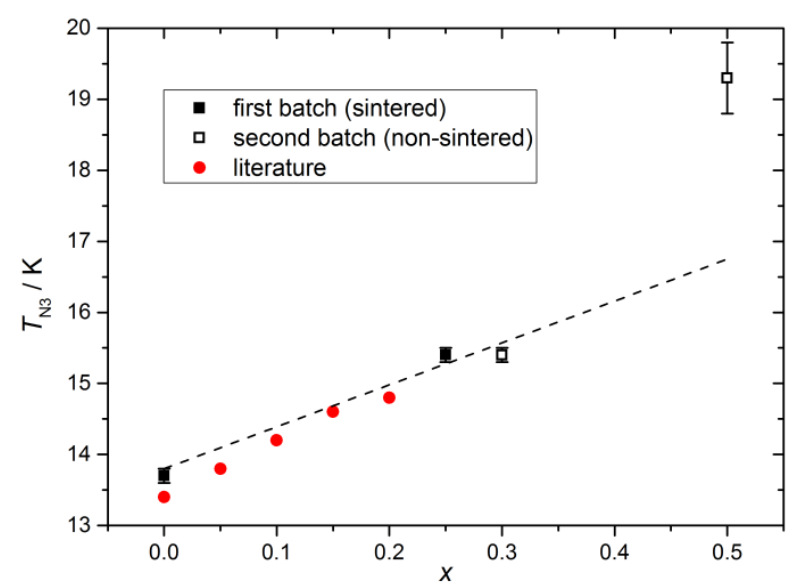

Figure 4-30: Dependence of the antiferromagnetic phase transition temperature $T_{\mathrm{N} 3}$ on the total amount of Mo. Closed black squares are data of sintered samples of the first batch while open black squares are results of the non-sintered samples of the second batch. The black dashed line is a guide to the eye for the linear relation of the $x \leq 0.3$ samples. Red dots are published data [63] for comparison.

While the magnetization of the $x=0.25$ sample of the first batch and of the $x=0.3$ sample of the second batch are similar, this not true for the $x=0.5$ samples of both batches. The magnetization and its temperature derivative of the first batch sample are presented in Figure 4-31 and Figure 4-32, respectively. In fact, this sample stands out from all other measured ones because the magnetization strongly increases during cooling around $T_{4} \approx 44 \mathrm{~K}$. Furthermore, there are three anomalies at the temperatures $T_{1}=8.9 \pm 0.1 \mathrm{~K}, T_{2}=16 \pm 1 \mathrm{~K}$ and $T_{3}=28 \pm 3 \mathrm{~K}$, where the slope of the magnetization changes.

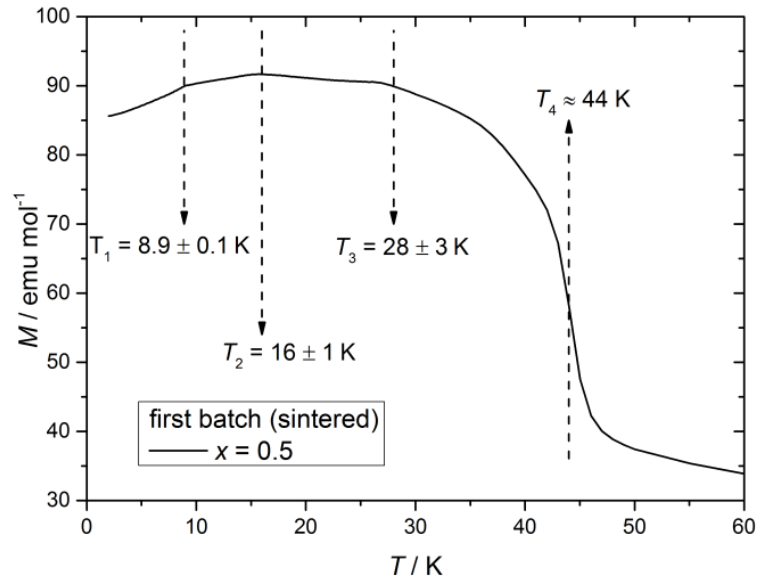

Figure 4-31: Temperature dependence of the magnetization of the sintered $x=0.5$ sample of the first batch for an external field of $H=1000$ Oe. Several anomaly temperatures are marked by black dashed lines.

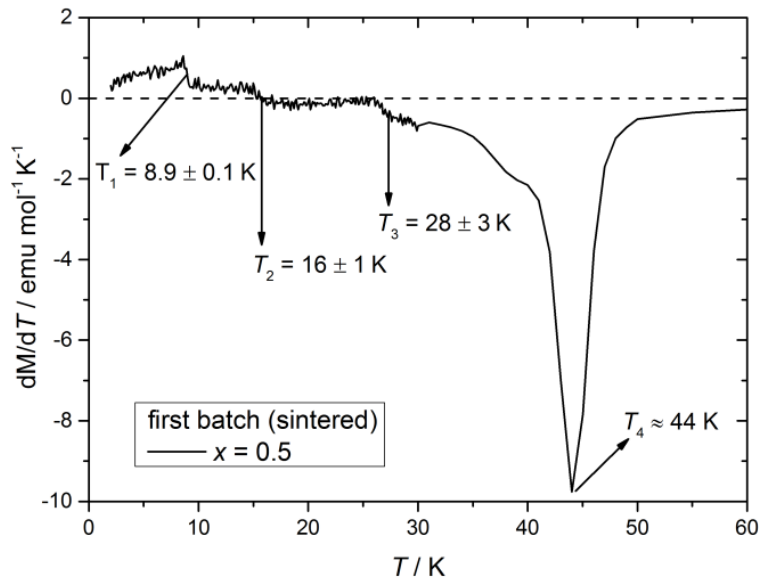

Figure 4-32: Temperature derivative of the magnetization of the sintered $x=0.5$ sample of the first batch for an external field of $H=1000$ Oe.

The strong increase of the susceptibility at $T_{4}$ coincides with the weak anomaly of the $x=0.25$, $x=0.5$ and $x=0.7$ samples. This increase might be an indication that a ferrimagnetically ordered phase appears where spins that are oriented in opposite directions do not cancel out, which results in a non-zero net magnetization. The behavior of such a material is similar to a ferromagnet, only the 
magnitude of the magnetization is significantly smaller. This static net magnetization $M_{(\mathrm{s})}$ is a primary ferroic according to Schmid (see chapter 2.1.2), while the magnetization that is induced by the external field $H$ via the susceptibility is a second order ferroic. If there is no $M_{(s)}$, the magnetic susceptibility for the present measurement is the ratio of the magnetization and the external field:

$$
M=\chi \cdot H \quad \chi=\frac{M}{H}
$$

The magnetization was therefore measured with external fields of different strength as illustrated in Figure 4-33 while the ratio of magnetization and field is presented in Figure 4-34. The latter is identical for all three fields at temperatures above $45 \mathrm{~K}$, which is an indicator that the magnetization is only induced due to magnetic susceptibility in this temperature range. This is different below $45 \mathrm{~K}$, where the absolute value of $M$ still significantly increases with $H$, but the ratio decreases with $H$. This is a sign that $M$ also includes static magnetization $M_{(s)}$ to a certain amount and the sample indeed experiences a ferrimagnetic phase transition at $45 \mathrm{~K}$.

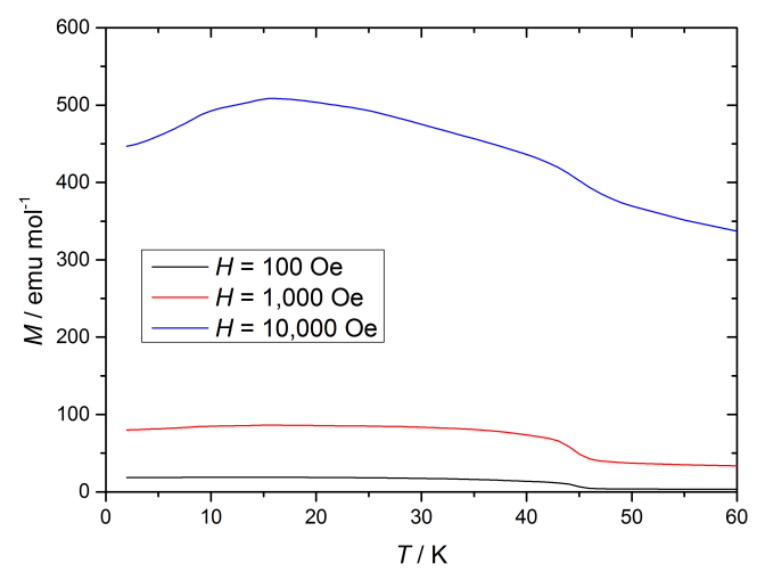

Figure 4-33: Temperature dependence of the magnetization of the sintered $x=0.5$ sample of the first batch for external fields of different strength.

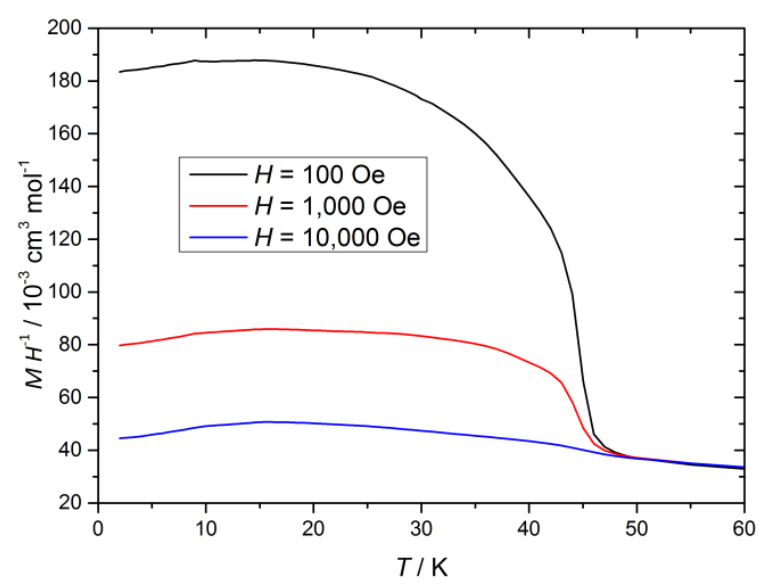

Figure 4-34: Temperature dependence of the magnetization divided by the strength of the external field for the sintered $x=0.5$ sample of the first.

At the end of chapter 4.3.3, the conclusion was drawn that there must be a difference between the $x \geq 0.3$ samples of the first and second batch induced by the variation of the reaction temperature, because the latter decompose during sintering while the first do not. The X-ray diffraction data could not answer this question, because the crystal structures seem to be identical. Measurements of the magnetic susceptibility, on the other hand, clearly indicate that the magnetic structures significantly differ, at least when comparing the sintered sample of the first batch with the non-sintered samples of the second batch. The ferrimagnetic order of the first batch samples can of course not explain why the metastable state survives sintering, because there is no magnetic order at $1100^{\circ} \mathrm{C}$. Nevertheless, it indicates that there is an intrinsic difference, which cannot be identified on the basis of the present experimental results. 


\subsection{Conclusion}

Mo-doped $\mathrm{MnWO}_{4}$ was synthesized during this thesis in order to clarify the inconsistencies of several publications about the phase diagram and the miscibility gap. The secondary aim was to determine the magnetic phase transition temperatures of all synthesized samples.

Two different ways of synthesis were tested, which mainly differ by the reaction temperature. The first batch was synthesized at $800^{\circ} \mathrm{C}$ and then sintered at $1100^{\circ} \mathrm{C}$. There is no effect of the sintering process according to X-ray diffraction results and it is possible to replace up to $65 \%$ of the tungsten atoms in the wolframite phase by molybdenum while no miscibility gap is observed. The second synthesis with a lower reaction temperature of $600^{\circ} \mathrm{C}$ also yields a wolframite phase with up to $65 \%$ molybdenum. The striking difference to the first batch is, however, that the samples exhibit clear signs of a phase separation during sintering. It seems that the Mo-rich wolframite phase is a metastable state that decomposes at $1100^{\circ} \mathrm{C}$ when synthesized at $600^{\circ} \mathrm{C}$ but remains stable when synthesized at $800^{\circ} \mathrm{C}$. However, a later attempt to reproduce the synthesis of the first batch failed and a phase separation occurred, too. Therefore, the reaction temperature might not be the relevant synthesis parameter that promotes the stability of the Mo-rich wolframite phase.

The magnetization of some Mo-rich wolframite samples was measured in order to determine the magnetic phase transition temperatures. The results of the $600^{\circ} \mathrm{C}$ sample with $x=0.5$ indicate that $T_{\mathrm{N} 3}$ indeed increases even stronger than the $x<0.3$ samples suggest. The $800^{\circ} \mathrm{C}$ sample with the same Mo-content, on the other hand, has different magnetic properties. There is a ferrimagnetic phase transition at approximately $44 \mathrm{~K}$ and three further transitions at lower temperatures. This underlines the observation that the metastable states of the first and second batch significantly differ, although the crystal structure is identical according to X-ray diffraction.

Another set of sintered tables for impedance spectroscopy exhibits a miscibility gap with a lower limit of $x_{1}=0.3$, which is consistent with the results of Meddar [18] and which might be the thermodynamic ground state at higher temperatures. This only leaves the conclusion that this thesis could just partially clarify the phase diagram of Mo-doped $\mathrm{MnWO}_{4}$. 


\section{Magnons in the Multiferroic Phase of CuO}

Dzyaloshinksi-Moriya interaction induces ferroelectricity in the antiferromagnetic AF2-phase of $\mathrm{CuO}$ and can therefore be regarded as the cornerstone of the multiferroic coupling. This interaction is expected to not only affect the static structure, but also to have an impact on spin dynamics. This became apparent when an electromagnon was discovered in the AF2-phase by THz-spectroscopy [20]. Electromagnons are magnetic excitations with partially phononic character. Like the spontaneous electric polarization is induced by static magnetic order, the oscillation of the magnetic structure can induce an oscillating electric dipole that in turn interacts with, for example, electromagnetic $\mathrm{THz}$-radiation. Electromagnons can therefore be regarded as elementary excitations of the multiferroic coupling. The determination of magnetic excitations can therefore be especially helpful when compared with similar measurements of the non-multiferroic AF1-phase.

Since THz-spectroscopy can only probe magnons at the magnetic $\Gamma$-point, inelastic neutron scattering is the next step for further investigations of the dispersion curve. Most inelastic magnetic measurements of $\mathrm{CuO}$ that have been published so far $[86,87,89,90]$ were, however, conducted either at temperatures well below $T_{\mathrm{N} 1}$ or in the paramagnetic phase above $T_{\mathrm{N} 2}$. This part of the thesis therefore aims at closing this gap by the measurement of magnon spectra in the multiferroic phase as well as comparative spectra in the AF1-phase. The new polarization analysis setup of PUMA@FRMII proofs to be well suited for this task since it can not only unambiguously distinguish the magnetic structure of both phases, but also characterize the eigenvector of all magnons.

The first two subchapters will provide an overview over experimental considerations regarding the sample preparation and the instrumental setup, respectively. The next subchapter presents measurements of magnetic superlattice reflections in both phases and in different Brillouin-zones. Their purpose is to confirm the magnetic structure of our specific $\mathrm{CuO}$ sample, but they also serve as a "proof of principle" for the polarization analysis setup since this is its first major experiment. Finally, the magnon spectra of both phases are presented in a further subchapter together with a conclusive interpretation of the results.

At this point, it shall be mentioned that another group published a similar study after the experiments of this thesis were finished [88]. Their research was independent from the present investigation, but their findings are consistent with the conclusions of this thesis. 


\subsection{Sample preparation}

The CuO single crystal used in this thesis is purchased from MaTeck $\mathrm{GmbH}$. It has a cylindrical shape with a diameter of $8 \mathrm{~mm}$ and a length of $30 \mathrm{~mm}$ and was cut by MaTeck along a plane perpendicular to the ( $\left.\begin{array}{lll}2 & 0 & 2\end{array}\right)$-vector as represented in Figure 5-1. The crystal consists of several grains with a mosaicity of a few ${ }^{\circ}$ as determined by $\gamma$-ray diffraction (see chapter 3.1.2). In order to reduce the mosaicity, a part at the tip of the crystal that is marked with grey color in Figure 5-1 was cut out of the crystal and used for further experiments.

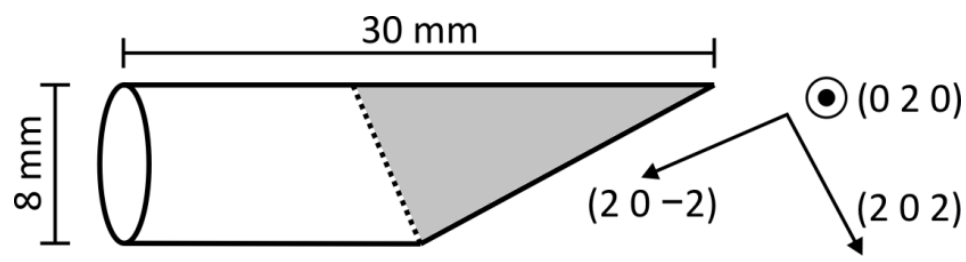

Figure 5-1: Schematic representation of the CuO single crystal used in this thesis. The grey colored part of the crystal is used for neutron scattering experiments. The dashed line marks the cut with a diamond wire saw.

The remaining mosaicity of the sample was determined by $\gamma$-ray diffraction, which is presented in Figure 5-2 using the lattice Bragg-reflections (2 02$)$ and $(20-2)$ as examples. The vertical axis corresponds to the $\omega$-angle which is varied during one scan. The horizontal slit in front of the sample is closed to $1 \mathrm{~mm}$. All detected intensity of one scan therefore originates from a thin slice of the sample. The sample is then translated perpendicular to the slit between two scans and the value of the translation corresponds to the horizontal axis. Figure 5-2 can therefore be interpreted as
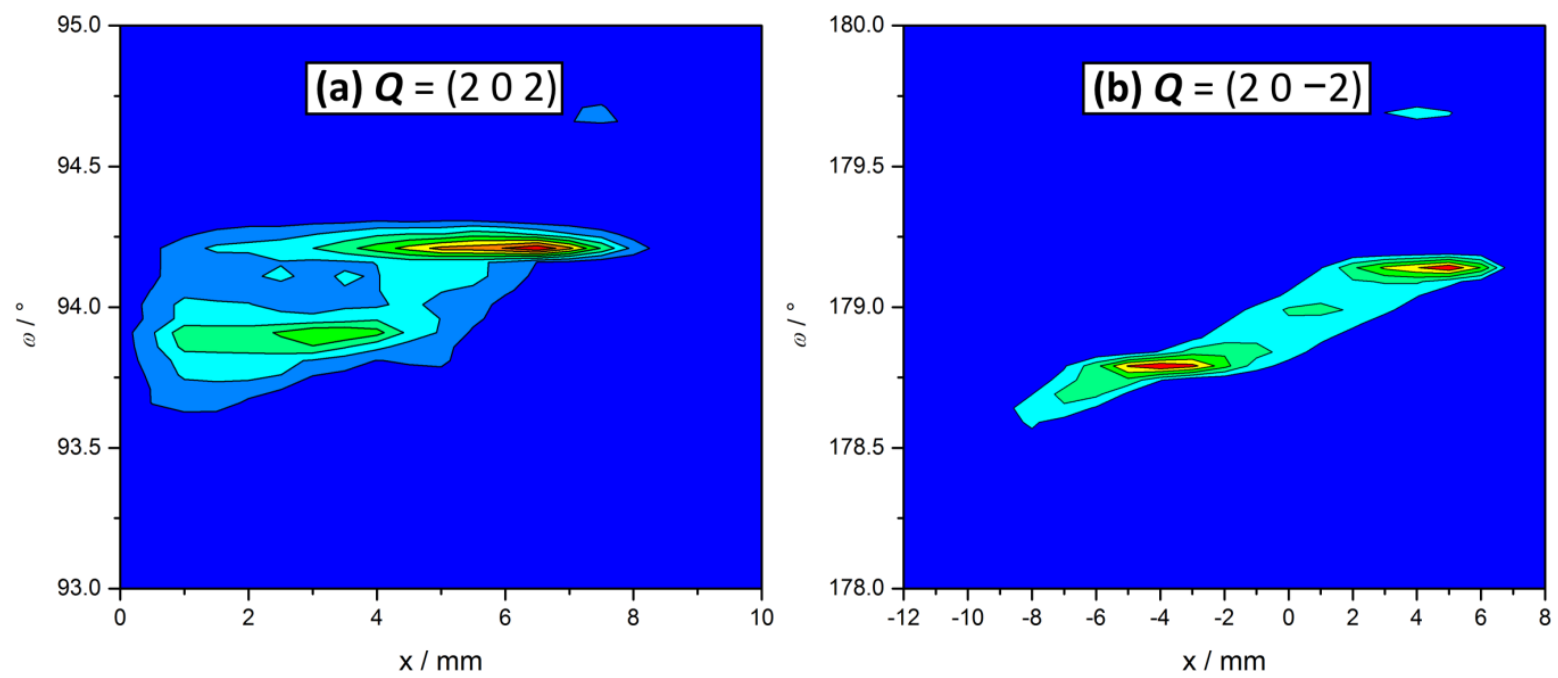

Figure 5-2: Contour plots of grid scans of the lattice Bragg-reflections (202) and (20-2). The $\omega$-angle (vertical axis) is varied during one scan while the whole sample is translated between two scans along an axis perpendicular to the horizontal slit in front of the sample. This slit is closed to about $1 \mathrm{~mm}$ so all detected intensity of one scan originates from a thin slice of the sample. The value of the translational axis corresponds to the horizontal axis of the contour plot. 
a 1D-spatially resolved representations of the mosaicity.

The translation axis of Figure 5-2-a is roughly perpendicular to the cylindrical axis. There are clearly two grains visible which are rotated by slightly less than $0.5^{\circ}$ to each other. This is different for the scans of Figure 5-2-b where the translation axis is roughly parallel to the cylindrical axis. There is only one grain which continuously rotates with increased translation. The total rotation of this grain in the final sample is $0.5^{\circ}$. In summary, the sample used for neutron scattering experiments has an effective mosaicity of around $0.5^{\circ}$.

The (2 0 -2)-plane of the sample is finally glued with the two component epoxy resin "Uhu plus schnellfest" to a sample holder made of aluminium. Pictures of the mounted sample are presented in Figure 5-3. The sample holder is partially covered by boron nitride to absorb neutrons scattered by polycrystalline Al. Sample and holder are installed on the bottom lid of a sample can which is then filled with He-gas for better heat conductivity and closed while the connection between sample can and bottom lid is sealed with an indium wire. The whole sample can is mounted to the cold head of a helium closed-cycle cryostat. The temperature of the cold head is measured with a Si-diode while a Pt100-thermometer is glued as close to the sample as possible inside the sample can (see Figure 5-3).
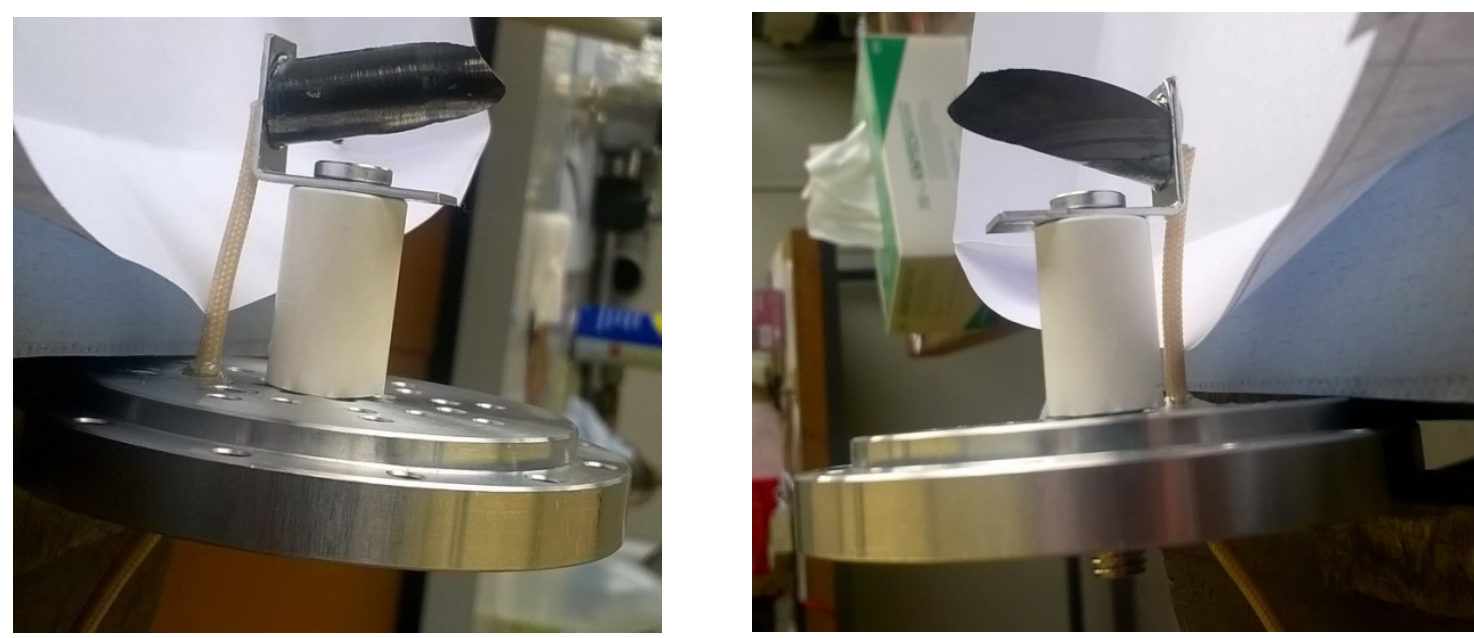

Figure 5-3: Picture of the CuO single crystal mounted to a sample holder on the bottom lid of the sample can. The beige wire belongs to a Pt100-thermometer which is glued as close to the sample as possible.

\subsection{Instrumental setup}

The new polarization analysis setup of PUMA, as described in chapter 3.1.5.2, is used for all neutron scattering experiments on $\mathrm{CuO}$ during this thesis. A 60'-collimator is placed between virtual source and monochromator $\left(\alpha_{1}\right)$ as well as between monochromator and sample $\left(\alpha_{2}\right)$. The collimator behind the sample is set to $\alpha_{3}=30^{\prime}$. The PG(002)-monochromator is vertically focused. The slits 
before and behind the sample are adjusted to the sample size while measuring a lattice reflection. This results in a horizontal slit width of $8 \mathrm{~mm}$ and a vertical one of $35 \mathrm{~mm}$. Constant- $k_{\mathrm{f}}$ configuration is used with $k_{\mathrm{f}}=2.662 \AA^{-1}$.

The experiment is divided into two parts. First are elastic measurements of the magnetic superlattice reflection in several Brillouin-zones and at different temperatures. This allows probing the static magnetic structure of $\mathrm{CuO}$. The second step is inelastic neutron scattering in order to characterize magnetic excitations. Due to the steep magnon dispersion $[86,89,90]$, these scans are also close to the magnetic satellite. It is therefore necessary to find a scattering setup which provides easy access to superlattice reflections in several Brillouin-zones. Since the satellites only have $a$ - and $c$-components, it is the obvious choice to use the $\boldsymbol{a}$-c $\boldsymbol{c}$-plane as scattering plane. The resulting selecting rules for SF- and NSF-scattering are sketched in Figure 5-4. SF-scattering corresponds to magnetization within the scattering plane perpendicular to the scattering vector $\boldsymbol{Q}$. It is therefore necessary to find two reflections with orthogonal scattering vectors in order to gain all possible information about magnetization in the $\boldsymbol{a}$-c-plane plane. NSF-scattering, on the other hand, is always a result of magnetization perpendicular to the scattering plane, independent of the orientation of $\boldsymbol{Q}$. In the present setup, this magnetization is parallel to the $\boldsymbol{b}$-axis.

This scattering setup allows the clear distinction of both antiferromagnetic phases because the primary easy-axis coincides with the $\boldsymbol{b}$-axis and therefore only contributes to NSF-scattering. The secondary easy-axis, on the other hand, lies in the a-c-plane and can only correspond to SF-scattering, depending on $\boldsymbol{Q}$. All three antiferromagnetic phases of $\mathrm{CuO}$ have spin components in $\boldsymbol{b}$-direction, but only AF2 exhibits magnetization along the secondary easy-axis. Elastic SF-scattering is therefore a direct probe of the AF2-phase. For this reason, the present setup was used for all neutron scattering experiments on $\mathrm{CuO}$.

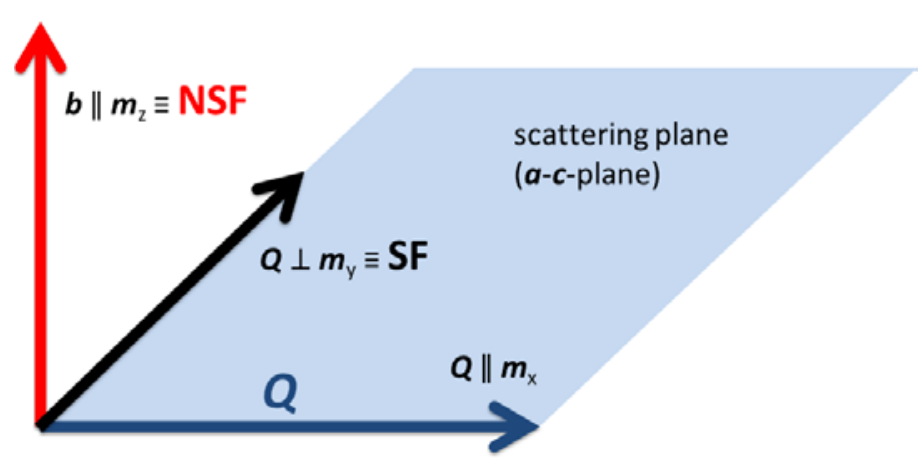

Figure 5-4: Schematic representation of the scattering setup. The scattering plane is equal to the crystallographic $\boldsymbol{a}$-c-plane. Spin-flip scattering corresponds to magnetization in the scattering plane perpendicular to the scattering vector $\boldsymbol{Q}$. Non-spin-flip scattering results from magnetization perpendicular to the scattering plane and thus parallel to the $\boldsymbol{b}$-axis. 


\subsection{Elastic measurements}

Since elastic NSF- as well as SF-scattering of the magnetic superlattice reflections depends on the static magnetic structure, the intensity of both spin channels is expected to vary between the AF1- and AF2-phase. This is reflected by longitudinal scans of the commensurate and incommensurate satellite in the $0^{\text {th }}$-Brillouin-zone in Figure 5-5-a and $c$, respectively. Since all magnetization in the AF1-phase is oriented along the $\boldsymbol{b}$-axis, only NSF-intensity is expected for the commensurate reflection (see Figure 5-5-a), independent of the direction of the scattering vector $\boldsymbol{Q}$. In the AF2-phase on the other hand, magnetization along the secondary easy-axis arises which can contribute to SF-intensity, but its magnitude depends on the current scattering geometry. In the case of the incommensurate reflection of Figure $5-5-c, Q$ is nearly perpendicular to the cycloidal plane (see Figure 5-5-d) and therefore NSF- as well as SF-scattering correspond to the magnetization of the spin-cycloid and should be equal because CuO has a perfectly circular spin cycloid [77]. In fact, the SF-intensity is slightly lower because the angle between $\boldsymbol{Q}$ and the scattering plane deviates from $90^{\circ}$.
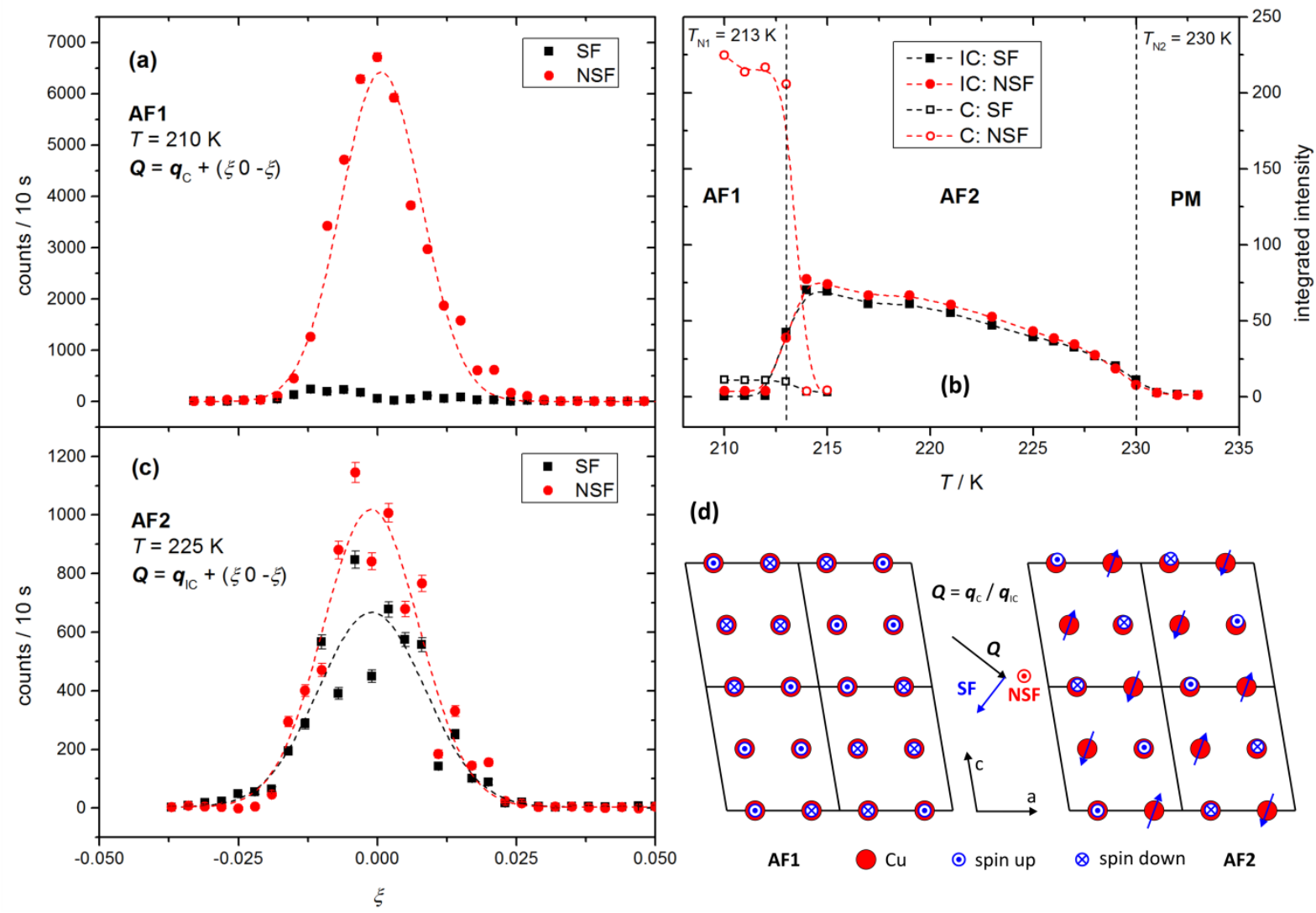

(d)

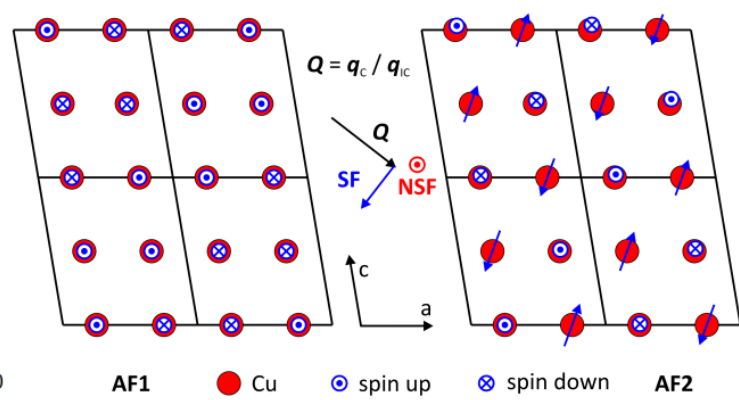

Figure 5-5: (a) and (c): Scans of the magnetic superlattice reflection in the AF1- and AF2-phase, respectively. (b): Integrated intensity of respective scans of the commensurate and incommensurate superlattice reflection at different temperatures. All lines in (a) - (c) are guides to the eye. (d): Orientation of the scattering vector relative to the magnetic structure. All intensities are corrected according to equations (3-6) and (3-7). 
In order to illustrate the temperature variation of the superlattice reflection, the integrated intensity of longitudinal scans of the commensurate as well as the incommensurate satellite in the 0.-Brillouin-zone at different temperatures is presented in Figure 5-5-b. The result is in accordance with a previous study by Forsyth who only detected the total scattered intensity though [75]. Below $213 \mathrm{~K}$, there is only intensity in the NSF-channel of the commensurate satellite which drops with onset of the AF2-phase while SF- and NSF-intensity of the incommensurate satellite arise. At $T_{\mathrm{N} 1}$, both phases coexist which reflects the first order nature of this phase transition. The intensity in the multiferroic AF2-phase steadily decreases upon heating while it is roughly proportional to the electric polarization $[19,81,91]$. The intensity does not abruptly disappear at $T_{\mathrm{N} 2}$ due to the second order nature of this transition, but it rather fades away with increasing temperature. The reflection is still detectable at $233 \mathrm{~K}$. It was reported in a previous study that the superlattice reflection persists to temperatures up to $400^{\circ} \mathrm{C}$ [78]. This, however, cannot be confirmed. A measurement of the magnetic satellite at room temperature yields no intensity except background.

The fact that the spontaneous electric polarization is proportional to SF- as well as NSF-intensity can be easily explained. According to equation (2-12) in chapter 2.2.1 which describes the spontaneous electric polarization induced by Dzyaloshinski-Moriya interaction, $\boldsymbol{P}$ is proportional to the square of the sublattice magnetization. The same is also true for the neutron intensity as described in chapter 3.1.5.1. Hence, $\boldsymbol{P}$ is proportional to the intensity.

The recently discovered antiferromagnetic phase AF3 exists in the temperature range between $T_{\mathrm{N} 2}=229.2 \mathrm{~K}$ and $T_{\mathrm{N} 3}=229.8 \mathrm{~K}$. The magnetic structure of this phase is not yet determined, but it is expected that all spins are oriented along the primary easy-axis with an incommensurate modulation of the amplitude, in analogy to the AF3-phase of $\mathrm{MnWO}_{4}$. This would result in pure NSF-scattering like in the case of AF1. At all temperatures between $233 \mathrm{~K}$ and $213 \mathrm{~K}$, however, the incommensurate satellite exhibits the same NSF to SF intensity ratio. The scans were made in $1 \mathrm{~K}$ steps at integer temperatures like $230.0 \mathrm{~K}$ and $229.0 \mathrm{~K}$, so none of the data points in Figure 5-5-b actually refers to AF3. It is therefore not possible to draw any conclusion about the magnetic structure of the AF3-phase from these results.

All considerations about elastic scattering so far only included magnetic satellites in the $0^{\text {th }}$-Brillouin-zone. This is insufficient for two reasons. SF-intensity for this orientation of $\boldsymbol{Q}$ can only probe magnetization along the secondary easy-axis, so all information about the remaining direction inside the $\boldsymbol{a}$-c-plane, the hard-axis, is missing. Furthermore, the absolute value of $\boldsymbol{Q}$ is so small that it only permits inelastic measurements with an energy transfer of up to $E \approx 8 \mathrm{meV}$. For magnon spectra with higher $E$, it is necessary to choose a superlattice reflection in a higher Brillouin-zone. In conclusion, in order to gain quantitative comparable magnon spectra that contain all possible 
information about magnetic excitations in the $\boldsymbol{a}$ - $\boldsymbol{c}$-plane, at least two Brilloun-zones are needed where the scattering vectors of the respective magnetic satellites are orthogonal.

The best choice to fulfill the above requirements are the $\left(\begin{array}{lll}0 & 0 & 2\end{array}\right)$ - and (-2 02 2)-Brillouin-zones. Figure 5-6-a and-b displays longitudinal scans of the commensurate and incommensurate satellite, respectively, of the $(-202)$-zone. Since the scattering vector of this zone is parallel to $Q$ of the $0^{\text {th }}$-zone (compare Figure 5-5-d and Figure 5-6-d), the selection rules are the same as described before. This is different, however, for the incommensurate satellite in the $(002)$-zone where $\boldsymbol{Q}$ is parallel to the cycloidal plane (see Figure 5-6-d). A scan of this reflection along the $(\xi 0-\xi)$-direction is presented in Figure 5-6-c. In this case, SF-scattering corresponds to magnetization perpendicular to the cycloid which is equivalent to the hard-axis. The SF-channel therefore detects nearly no neutrons since there are no spins oriented in this direction.

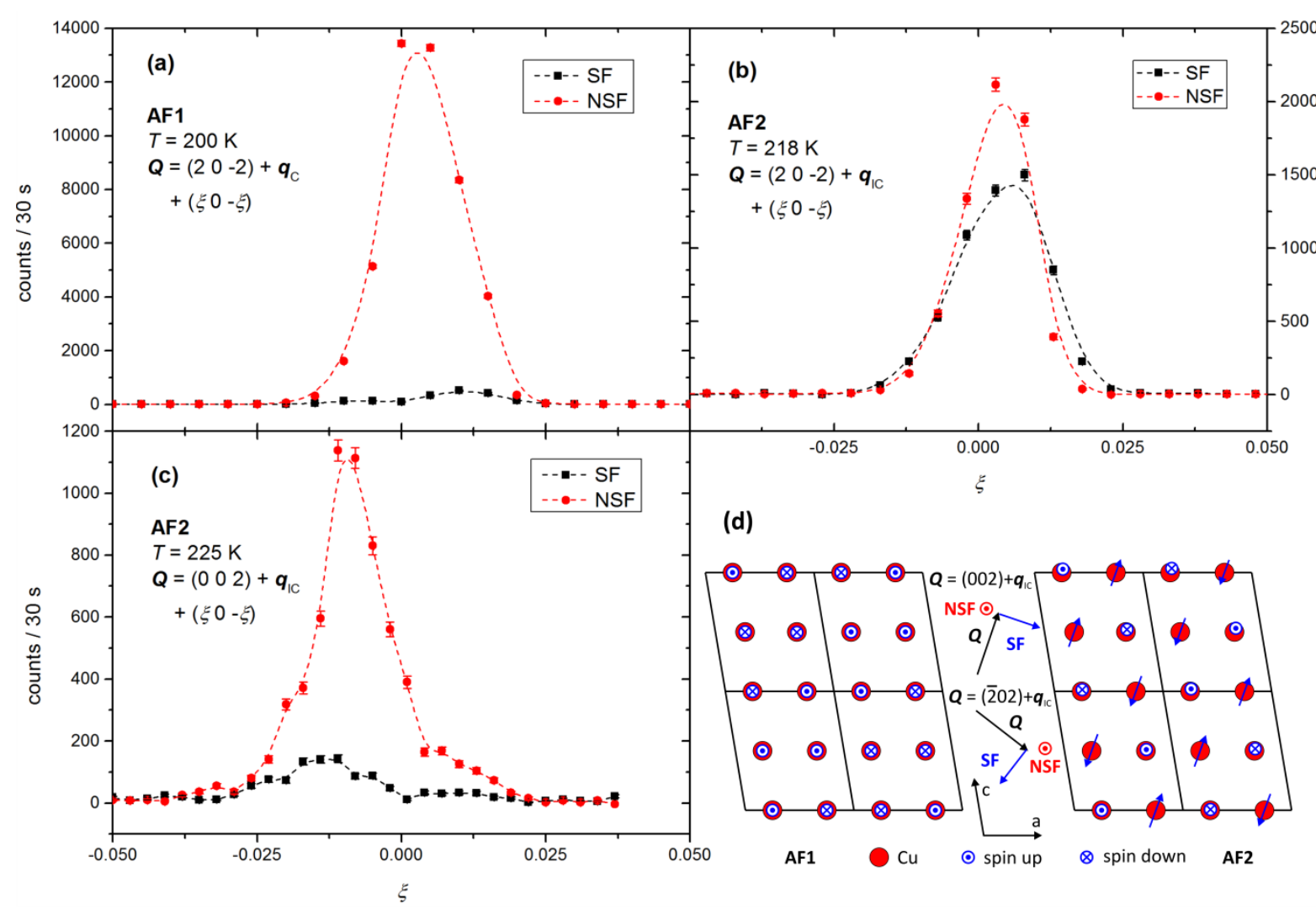

Figure 5-6: (a-c): Scans of magnetic superlattice reflections in different antiferromagnetic phases and Brillouin-zones. All lines are guides to the eye. (d): Orientation of the scattering vector relative to the magnetic structure. All intensities are corrected according to equations (3-6) and (3-7). 


\subsection{Magnon spectra}

There are several publications about the magnon dispersion of $\mathrm{CuO}$ in the AF1-phase $[86,89,90]$. Its slope close to the magnetic $\Gamma$-point is so steep that different magnon branches cannot be resolved due to the restricted resolution of the experimental setup used in this thesis. This is exemplified by several constant energy scans in Figure 5-7-a-c. Please note that an unpolarized neutron beam was used, because these measurements are fast preliminary scans in order to probe the dispersion. Every figure displays a broad intensity distribution with the center at the position of the superlattice reflection. The intensity may not only correspond to different magnon branches, but also to different propagation directions of the same branch. This is schematically depicted in Figure 5-7-d by blue arrows that mark the path of the resolution ellipsoid through the dispersion curve. Nevertheless, it is possible to gain qualitative information about the slope of the dispersion in different propagation directions as shown in Figure 5-7-a. Both measurements were conducted with the same energy transfer and temperature, but the scanning direction differs. The scan along $\left(\begin{array}{lll}1 & 0 & 1\end{array}\right)$ is much broader compared to the (10-1)-direction, allowing the conclusion that the slope of the

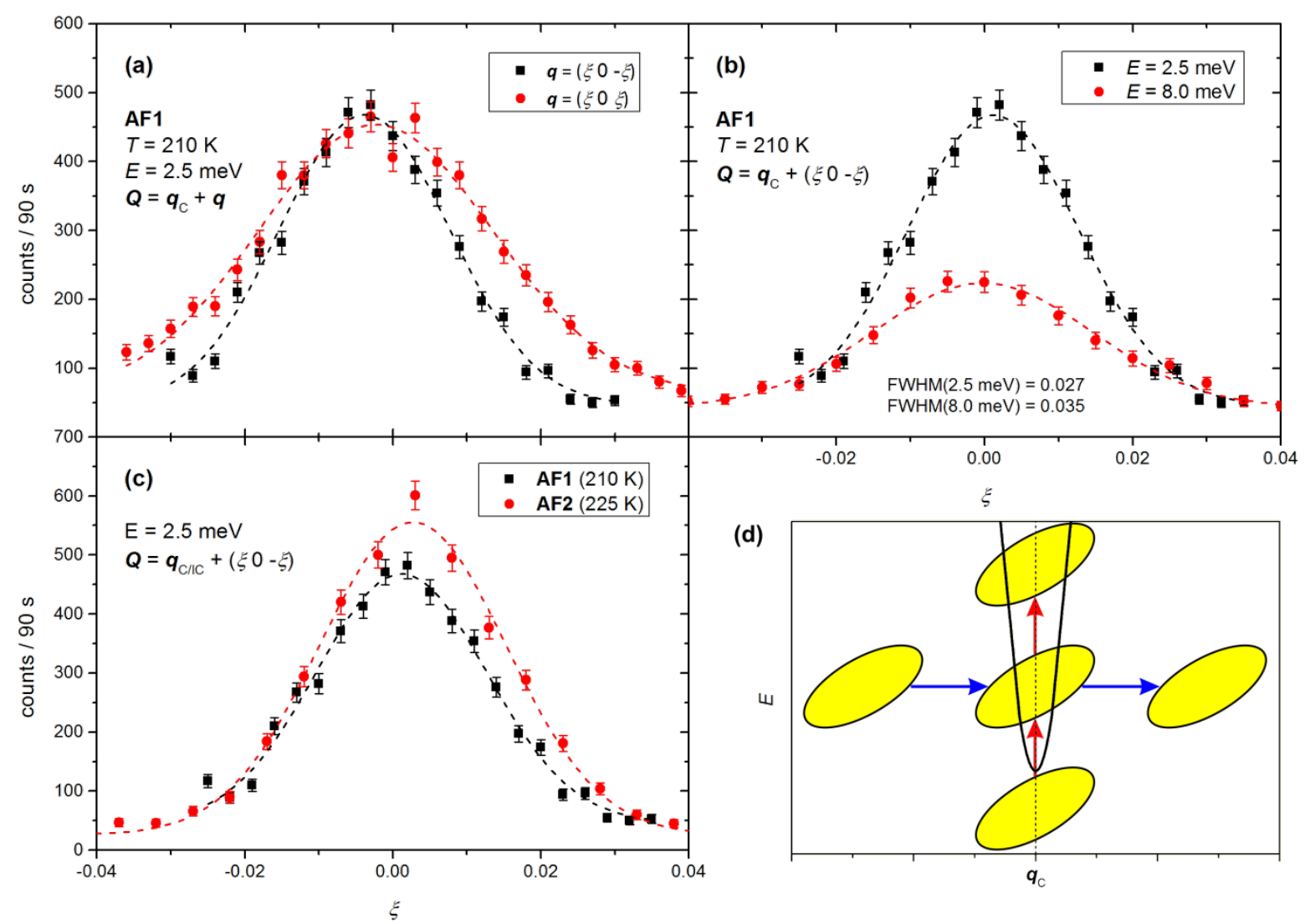

Figure 5-7: $(a-c)$ : Constant energy scans with an unpolarized neutron beam in order to compare two magnons with different properties. Dashed lines are Gauß profiles determined by a least-squares fit. (a): Magnons with the same energy transfer in the AF1-phase, but with different propagation directions. (b): Magnons with the same propagation direction in the AF1-phase, but with different energy transfers. (c): Magnons with the same energy transfer and propagation direction, but in different phases. (d): Schematic representation of the resolution ellipsoid (yellow) and the magnon dispersion curve at low energy transfer. Blue arrows mark constant energy scans and red arrows correspond to constant- $\boldsymbol{q}$ scans. All intensities are corrected according to equations (3-6) and (3-7). 
magnon branch is anisotropic and steeper along $\left(\begin{array}{lll}1 & 0 & -1\end{array}\right)$ than $\left(\begin{array}{lll}1 & 0 & 1\end{array}\right)$. This is consistent with published results $[86,89,90]$. An increase of the full width at half maximum (FWHM) is also observed at higher energy transfer $E$ as shown in Figure 5-7-b where one of the scans of Figure 5-7-a with $E=2.5 \mathrm{meV}$ is compared with the equivalent measurement at $E=8.0 \mathrm{meV}$. This can simply be explained by the fact that the dispersion curves diverge from the superlattice reflection with increasing energy and the detected intensity is therefore spread over a larger part of the scanning trajectory.

Figure 5-7-c compares preliminary measurements of the AF1- and AF2-phase, respectively. Despite the considerable differences of the magnetic structure in both phases, the scans are qualitatively identical. This is also true for further scans with different energy transfer and in other Brillouin-zones. The general shape of the magnon dispersion is unaffected by the phase transition. The slope does not significantly change and no additional low-lying magnon branches were found in this Brillouin-zone. For the measurements with polarized neutron beam, which have nearly one order of magnitude less scattered intensity and therefore consume most of the precious beam time, constant- $\boldsymbol{q}$ scans are conducted because they still yield information about the eigenvector of the magnons, but need less scan points than consecutive constant-E-scans.

As explained in the previous chapter, there are scans in two different Brillouin-zones for each phase to account for the specific selection rule of the SF-channel. Beginning with the AF1-phase, the spectrum in the $\left(\begin{array}{ll}0 & 0\end{array}\right)$-zone exhibits only background intensity for the NSF-channel (see Figure 5-8-a). All spins in this phase are oriented along the $\boldsymbol{b}$-axis and can therefore only be displaced inside the scattering plane. Hence, only SF-intensity can be detected in this phase independent of the scattering vector. The intensity starts to rapidly increase above $3 \mathrm{meV}$ which indicates that there is a spin gap in the magnon dispersion.

The spin gap is the energy minimum of the dispersion curve that is located at the magnetic $\Gamma$-point (see Figure 5-7-d). At this point, the displacement pattern has an infinite wave length, which means that the distortion of all spins has the same phase. This is equal to a uniform displacement of the whole magnetic structure relative to the lattice and therefore involves only anisotropic superexchange interactions and single ion anisotropy. At another point of the dispersion with non-vanishing $\boldsymbol{q}$, there is a phase shift introduced between neighboring spins, which increases with $\boldsymbol{q}$. This induces a relative tilting between these spins and therefore the isotropic exchange interactions become increasingly relevant. This also explains the steep slope of the dispersion curve, because $\mathrm{CuO}$ exhibits an exceptionally high exchange integral in [ $\left[\begin{array}{lll}1 & 0 & 1\end{array}\right]$-direction.

After a maximum around $5 \mathrm{meV}$, the intensity gradually decreases which can be explained by two factors. First, the intensity of the scattered neutron beam decreases exponentially with higher 
energy transfer. Second, there is less overlap of the magnon branches with the resolution ellipsoid at higher energies (see Figure 5-7-d). This continuous decrease in intensity is observed for all measured magnon branches.

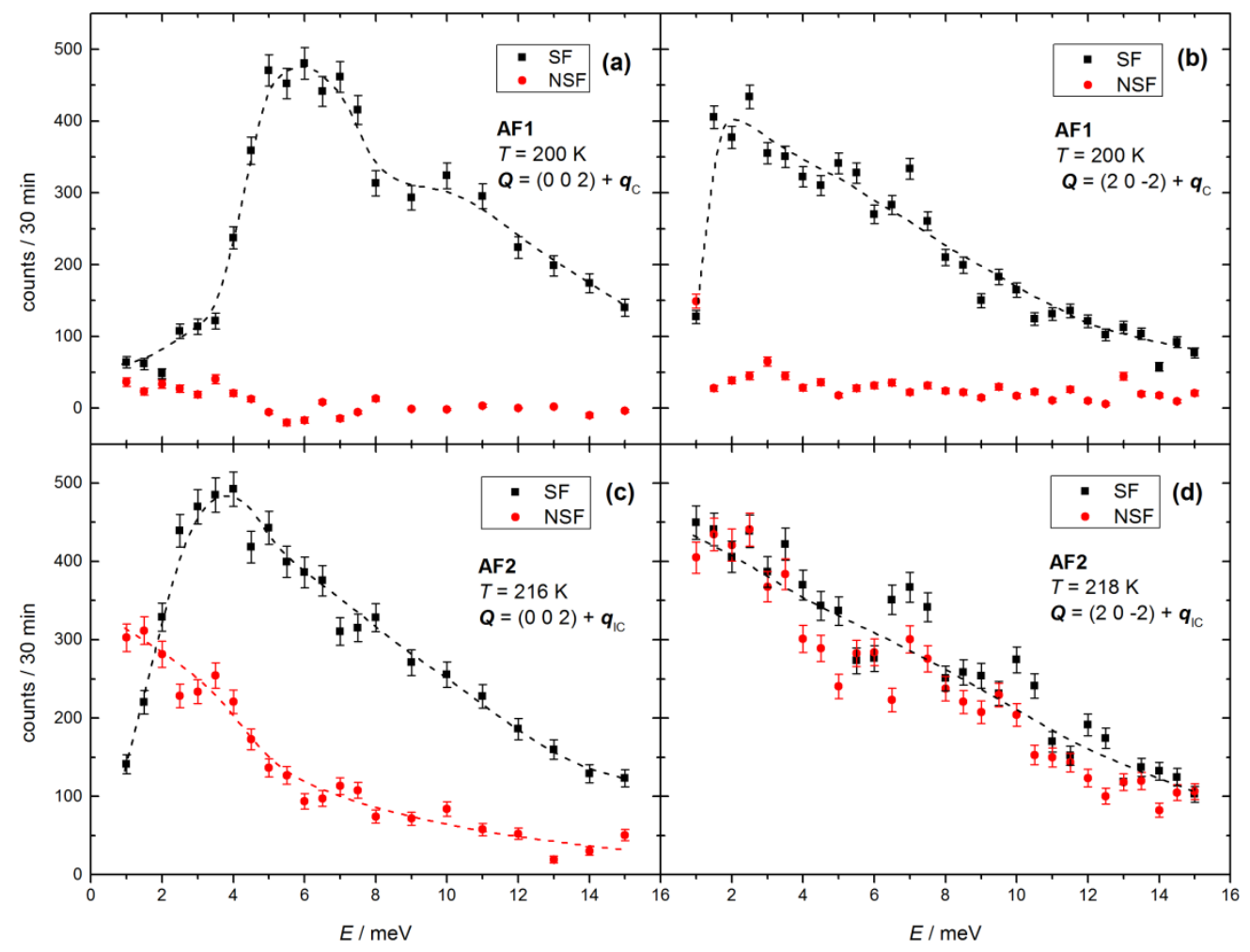

Figure 5-8: Constant- $Q$-scans at the magnetic superlattice reflection in both antiferromagnetic phases and two different Brillouin-zones. Dashed lines are guides to the eye. All intensities are corrected according to equations (3-6) and (3-7).

The spectrum in the (20-2)-Brillouin-zone is nearly identical (see Figure 5-8-b), but with a smaller spin gap. In this case, the maximum intensity is already reached around $2 \mathrm{meV}$. The different spin gaps can be explained by the orientation of the scattering vector (see Figure 5-6-d). In the (2 0 -2)-zone, $\boldsymbol{Q}$ is nearly perpendicular to what becomes the cycloidal plane in the AF2-phase. All SF-intensity can therefore be attributed to the displacement of spins along the secondary easy-axis, which is a magnetically preferred direction. This is energetically more favorable compared to the ( 00 2)-Brillouin-zone, where the scattering vector is parallel to the secondary easy-axis and the spins are therefore distorted perpendicular to the cycloidal plane along the hard-axis. Hence, the spin gap is larger in this case.

The magnon spectrum of the multiferroic AF2-phase in the $\left(\begin{array}{lll}0 & 0 & 2\end{array}\right)$-Brillouin-zone shows two different modes with distinct characteristics (see Figure 5-8-c). The SF-spectrum is similar to that of the AF1-phase with a maximum around $4 \mathrm{meV}$ and a gradual decrease in intensity with higher energy transfer. Since the scattering vector is pointing along the secondary easy axis, this mode can be 
associated to a distortion of spins orthogonal to the cycloidal plane. The NSF-channel, on the other hand, has no intensity maximum and therefore no spin gap within the experimental resolution. This mode is a displacement of spins along the $\boldsymbol{b}$-direction inside the cycloidal plane. The lack of a spin gap can also be found in both spin channels of the spectrum in the (2 0 -2)-Brillouin-zone (see Figure 5-8(d)) where $\boldsymbol{Q}$ is perpendicular to the cycloidal plane. NSF- as well as SF-intensity can be attributed to a spin displacement inside the cycloidal plane. All spectra are in qualitative agreement with a recent inelastic neutron scattering study that measured similar magnon spectra in the AF1- as well as the AF2-phase [88]. That study was conducted independently of this thesis and the results were published after the present experiments were finished.

All three channels that exhibit no spin gap correspond to the same magnon, which is a sliding mode of the spin cycloid. At the magnetic zone-center, this is equivalent to a phase shift of the whole cycloid with respect to the crystallographic lattice (see Figure 5-9) and therefore called phason. This is an energy-free excitation in the case of a non-elliptic cycloid like CuO [77], explaining the lack of a spin gap. Both intensities in Figure 5-8(d) are equal due to the perfect circular shape of the spin cycloid. This kind of magnetic excitation is unique for materials with cycloidal spin order and was first described by Katsura [122]. A phason mode was identified by polarized neutron scattering in $\mathrm{TbMnO}_{3}$ which also has a cycloidal magnetic structure like $\mathrm{CuO}[123]$.

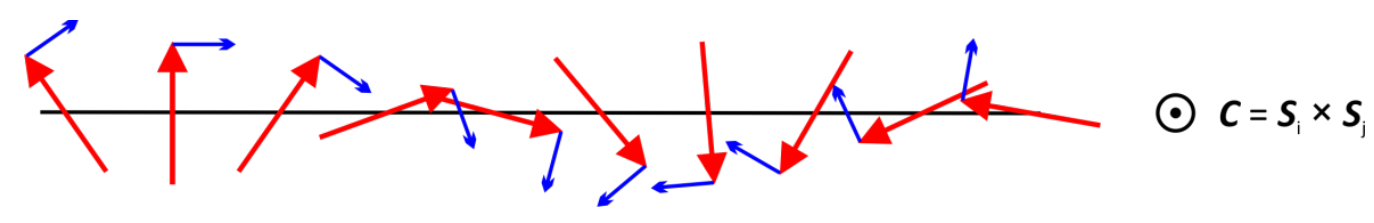

Figure 5-9: Schematic 1D-representation of the displacement pattern of the phason mode at the magnetic $\Gamma$-point. The image plane is equal to the cycloidal plane. Red arrows represent spins while the displacement direction of spins during the excitation is marked by blue arrows.

There are two other possible magnetic excitations of a spin cycloid whose displacement pattern can be imagined as a rotation of the cycloidal plane along different axes perpendicular to the cycloidal rotation vector $\boldsymbol{C}=\boldsymbol{S}_{\mathrm{i}} \times \boldsymbol{S}_{\mathrm{j}}$ [122,123] (see Figure $5-10$ ). This implies in both cases a displacement of spins perpendicular to the cycloidal plane, being responsible for the SF-intensity of Figure 5-8(c). The spin gap of $3 \mathrm{meV}$ corresponds to the energy of the magnon branch at the $\Gamma$-point, which is in accordance with the energy of the electromagnon found in CuO [20]. This was detected with THz-spectroscopy, which is an optical method that probes the magnetic zone center. A theoretical investigation [124] came to the conclusion that this mode is the rotation of the cycloidal plane along the secondary easy-axis (see upper panel of Figure 5-10) while the second mode is a rotation along the $\boldsymbol{b}$-axis (see lower panel of Figure $5-10$ ), which is also predicted to be an 
electromagnon. The reason why these two modes are electromagnons and the phason is not can be explained by the nature of the Dzyaloshinksi-Moriya interaction, which is described as:

$$
\boldsymbol{P} \propto \boldsymbol{k} \times\left(\boldsymbol{S}_{\mathrm{i}} \times \boldsymbol{S}_{\mathrm{j}}\right)=\boldsymbol{k} \times \boldsymbol{C}
$$

An oscillation of $\boldsymbol{P}$ therefore requires that the spins are distorted in such a way that the cycloidal rotation vector $\boldsymbol{C}$ is altering during the magnetic excitation. Consequently, a phason mode cannot be an electromagnon because all spins are equally rotated along $\boldsymbol{C}$ (see Figure 5-9), so $\boldsymbol{C}$ does not change. Both other modes, however, involve the rotation of the cycloidal plane and therefore also a displacement of $\boldsymbol{C}$ itself, which is displayed in Figure 5-10 as blue arrow. Hence, both magnons are accompanied by an oscillating electric dipole.

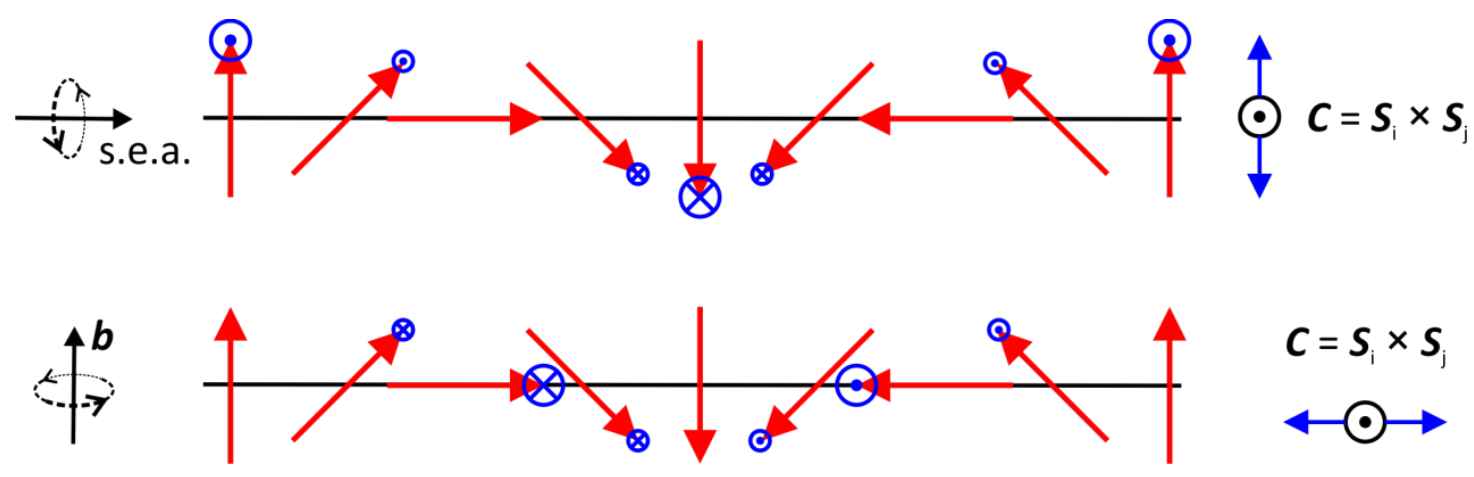

Figure 5-10: Schematic representation of the displacement pattern of both electromagnons. The image plane is equal to the cycloidal plane. Red arrows represent spins. The black vectors on the left are the rotation axes of both modes while blue dots and crosses are the resulting spin displacements in both directions perpendicular to the cycloidal plane. The change of the cycloidal rotation vector $\boldsymbol{C}$ is indicated by blue arrows on the right. The upper panel displays the mode at $E=3 \mathrm{meV}$ with a rotation of the cycloidal plane along the secondary easy-axis, according to [124], while the other mode at $E=13 \mathrm{meV}$ with a rotation along the $\boldsymbol{b}$-axis is shown in the lower panel.

The energy of the second electromagnon at the magnetic $\Gamma$-point is calculated to be $13 \mathrm{meV}$ [124]. This would result in another maximum in the SF-channel of Figure 5-8-c at this energy, but unfortunately there is no evidence for a second excitation. Maybe, this mode is a weak scatterer in this particular Brillouin-zone or the predicted energy is slightly underestimated. Only $3 \mathrm{meV}$ more and it would be outside the measurement range of Figure 5-8-c. 


\subsection{Conclusion}

The determination of magnons in the multiferroic phase of $\mathrm{CuO}$ is the first major experiment that was conducted with the new polarization analysis setup of PUMA@FRMII. The measurement of magnetic superlattice reflections in the antiferromagnetic AF1- and AF2-phases proved that the instrument is working as intended and also verified the magnetic structure of the sample. Furthermore, preliminary scans with unpolarized neutron beam confirmed that the magnon dispersion in the AF2-phase is similar to that of the AF1-phase in so far that the slopes do not significantly differ and there are no additional low-lying magnon branches.

The main part of the experiment is the determination of all magnons close to the $\Gamma$-point with an energy transfer of up to $15 \mathrm{meV}$. The polarization analysis allows an unambiguous assignment of all magnon eigenvectors, which are substantially different in both antiferromagnetic phases. In the AF1-phase, all magnetic excitations correspond to a displacement of spins perpendicular to the $\boldsymbol{b}$-axis, which is in accordance to the static structure of this phase where all spins are either parallel or antiparallel to the $\boldsymbol{b}$-axis. An anisotropic spin-gap is observed, which is smaller for spins that are distorted along the secondary easy-axis and higher for a displacement along the hard-axis.

In the AF2-phase, on the other hand, two distinct excitations are identified. The first one is a phason mode where the spins rotate inside the cycloidal plane and that has no spin-gap within the instrumental resolution. At the magnetic $\Gamma$-point, this is equivalent to a phase shift of the whole cycloid with respect to the crystallographic lattice, hence the name.

The second mode might be the electromagnon that was recently discovered by THz-spectroscopy. The determined eigenvector matches the predicted displacement pattern of the electromagnon, which is a rotation of the whole cycloidal plane along the secondary easy-axis. Furthermore, THz-spectroscopy determined the energy at the magnetic $\Gamma$-point to be $3 \mathrm{meV}$. This is exactly the value for the spin-gap of the second mode. Both observations are clear indicators that this mode is indeed the electromagnon, but it is no conclusive evidence, since there is no way to distinguish common magnons from electromagnons with inelastic neutron scattering. 


\section{Dielectric and Mechanical Investigation of BMO}

There are two important but yet unanswered questions regarding the multiferroicity of BMO. First, is BMO multiferroic at all? As mentioned in chapter 2.3.3.3, there is no experimental evidence for a spontaneous polarization until today. BMO is believed to be multiferroic, because all members of the $\mathrm{R}_{2} \mathrm{Mn}_{4} \mathrm{O}_{10}$-family exhibit ferroelectricity and have the same crystal structure. Therefore, impedance spectroscopy was employed in this thesis in order to determine the dielectric permittivity at the multiferroic phase transition. If there is any magnetoelectric effect in the multiferroic phase, the permittivity should change when the magnetic order appears. Especially in the case of ferroelectricity, a distinct anomaly of the permittivity is expected at the temperature of the ferroelectric phase transition $T_{C}$, which is equal to $T_{\mathrm{N}}$ in type-II multiferroics.

The second question is about the origin of the multiferroic coupling in $\mathrm{BMO}$, if there is any. Chapon proposed a mechanism that describes the occurence of ferroelectricity in $\mathrm{Tb}_{2} \mathrm{Mn}_{4} \mathrm{O}_{10}$ (for more details see chapter 2.3.3.3). This mechanism is based on magnetostriction where the onset of the magnetic order deforms the coordination geometry of $\mathrm{Mn}^{5+}$-pyramids and thereby breaks inversion symmetry. Since the crystal structure is distorted by magnetoelastic interactions, the mechanical behavior will be affected and, consequently, the elastic constants will change. Therefore, the sound velocities of different acoustic phonons were determined by inelastic neutron scattering during this thesis, first at room-temperature and later in the vicinity of $T_{\mathrm{N}}$. These results allow the calculation of elastic constants and can provide evidence whether the theory of Chapon can be applied to BMO.

This chapter is structured as follows: The preparation of the sample crystals is described in the first subchapter while the second subchapter characterizes the instrumental setup of both employed methods. The third and fourth subchapters are dedicated to the impedance spectroscopy and inelastic neutron scattering measurements itself, respectively. An evaluation of the data and an interpretation of the results will be provided. Finally, there are some concluding remarks in the last subchapter, how far the present investigations on BMO can answer the two initial questions. 


\subsection{Sample preparation}

Three different single crystals were used for the experiments on BMO. They were kindly provided by Prof. Gesing from Universität Bremen and were grown by the Top Seed Solution Growth technique (TSSG) as described in [95]. The mosaicity of all samples was determined by $\gamma$-ray diffraction to be less than $0.1^{\circ}$ (see chapter 3.1.2).

The crystal that was used for inelastic neutron scattering had to be glued onto an aluminium sample holder. In order to achieve a proper adhesion, it is necessary that the sample has a smooth surface parallel to the chosen scattering plane. Therefore, the sample was oriented with the $\gamma$-ray diffractometer and cut with a diamond wire saw in such a way that there are three planes perpendicular to the principal crystallographic axes. The resulting volume of the crystal was approximately $10 \times 10 \times 3 \mathrm{~mm}^{3}$.

The three samples of different orientation that were used for impedance spectroscopy are placed between the plates of a capacitor and therefore need two smooth surfaces that are perpendicular to the applied electric field. They were oriented and cut with the same technique as the INS-crystal. The dimensions and volumes of all three samples are listed in Table 6-1. Please note that the two samples that were used for measurements with field in $\boldsymbol{a}$ - and $\boldsymbol{b}$-direction were cut out of one crystal while the sample for the measurement in c-direction was part of another crystal.

Table 6-1: Dimensions of the three samples that were used for impedance spectroscopy. The first column denotes the direction of the applied electric field in the respective experiments. The $\boldsymbol{x}$ - and $\boldsymbol{y}$-axes span the area parallel to the plates of the capacitor while the $z$-direction is parallel to the electric field.

\begin{tabular}{|l|c|c|c|c|}
\hline & $x / \mathrm{mm}$ & $y / \mathrm{mm}$ & $z / \mathrm{mm}$ & $V / \mathrm{mm}^{3}$ \\
\hline $\boldsymbol{a}$-direction & 4.63 & 2.91 & 1.23 & 16.6 \\
\hline $\boldsymbol{b}$-direction & 4.56 & 2.50 & 1.77 & 20.2 \\
\hline $\boldsymbol{c}$-direction & 6.57 & 3.19 & 1.31 & 27.5 \\
\hline
\end{tabular}




\subsection{Instrumental setup}

\subsubsection{Impedance spectroscopy}

All impedance spectra of BMO can be divided in two distinct groups. The first series of measurements was obtained with high voltage decoupling device, as explained in chapter 3.2.1, in a temperature range of $300-2 \mathrm{~K}$. The spectra were taken while the temperature was continuously changed with different rates of $0.2-2 \mathrm{~K} \mathrm{~min}^{-1}$ in several alternating cycles of cooling and heating. Data for all three crystallographic directions were collected. The investigated frequency range of each spectrum is $100 \mathrm{~Hz}-1 \mathrm{MHz}$. The anomaly of the dielectric permittivity at $T_{\mathrm{N}}$, which is presented in chapter 6.3.1, was measured during this first series.

The measurements in the temperature range between 300 and $150 \mathrm{~K}$ were repeated without high voltage decoupling (see chapter 3.2.2) in order to extend the frequency range of this second series of measurements to $1 \mathrm{~Hz}-1 \mathrm{MHz}$. The duration of one repoling cycle of the applied AC voltage $U_{0}$ with the lowest frequency is $1 \mathrm{~s}$. In order to achieve a reasonable signal-to-noise ratio, the results of several cycles must be averaged. The integration time for a measurement at one frequency is therefore increased to $60 \mathrm{~s}$ while the delay between successive measurements is $1 \mathrm{~s}$. Since one spectrum takes half an hour with these settings, the temperature was kept constant during that time and only changed between the acquisitions of spectra. The integration time puts a lower limit to the accessible frequency range because an extension to $0.1 \mathrm{~Hz}$ would increase the necessary time by one order of magnitude. The measurements of just one direction would then need about half a year, which is obviously unfeasible. The results of this second series of measurements are presented in chapter 6.3.2.

\subsubsection{Inelastic neutron scattering}

The inelastic neutron scattering experiments on BMO can be divided in two parts. First are the measurements at room-temperature, which were conducted at PUMA@FRMII in a series of shorter experiments with internal beam time. The low-temperature investigations, on the other hand, took place at two different instruments: at PUMA@FRMII with the proposal number 11884 and at IN8@ILL with the experiment number 7-02-164 [125]. PUMA was operated in standard setup for all experiments on BMO.

Constant- $k_{\mathrm{f}}$-configuration with $k_{\mathrm{f}}=2.662 \AA^{-1}$ was used for all scans at both instruments. For the measurement of transverse phonons at room-temperature, the PG(002)-monochromator and analyzer in doubly focused mode was chosen, while collimation with only vertically focused PG(002)monochromator and analyzer is preferable for longitudinal phonons (for detailed explanation see 
chapter 6.4.1). The best compromise between intensity and resolution is achieved with $\alpha_{1}=40^{\prime}$, $\alpha_{2}=60^{\prime}, \alpha_{3}=45^{\prime}$ and $\alpha_{4}=45^{\prime}$. These values for collimation were also used for all low-temperature measurements at PUMA. Since the resolution of the focused monochromator at IN8 is too low for an accurate determination of acoustic phonon dispersions, all measurements at this instrument were conducted with a collimation of $\alpha_{1}=30^{\prime}, \alpha_{2}=30^{\prime}, \alpha_{3}=40^{\prime}$ and $\alpha_{4}=40^{\prime}$.

Three scattering planes were used for all experiments: $\boldsymbol{a} \boldsymbol{b}$-, $\boldsymbol{a} \boldsymbol{c}$ - and $\boldsymbol{b c}$-plane. In order to save precious neutron beam time, the sample was oriented with the $\gamma$-ray diffractometer towards the $\boldsymbol{a b}$-plane. For this purpose, the crystal was glued onto an aluminium sample holder with the instant adhesive Loctite 401 and placed on a small, portable goniometer, which is then mounted to the sample table of PUMA. In order to access the other two scattering planes, the goniometer was attached to a $90^{\circ}$-angle made out of aluminium. This setup, however, does not fit into a cryostat and cannot be used for low-temperature measurements. The crystal was therefore re-glued every time when the scattering plane must be changed. Viscous conducting silver paste was used as adhesive, which is suitable for low-temperatures and allows an easy dismounting. The low-temperature experiment at PUMA focused entirely on the $\boldsymbol{a c}$-plane, while the $\boldsymbol{b c}$ - and $\boldsymbol{a b}$-plane were investigated during beam time at IN8. 


\subsection{Dielectric investigation}

The results of the impedance spectroscopy experiments on BMO will be presented in this chapter, which is divided in two parts. First is the main part that focuses on the anomaly of the dielectric permittivity at the multiferroic phase transition temperature $T_{\mathrm{N}}$. There is a description of the experimental efforts that are necessary to detect this anomaly and which may also explain why there are no reports about its observation until today. In order to check the measurements for consistency with published data, the temperature- and frequency dependence of Debye relaxations along all three crystallographic main directions in a temperature range between 150 and $300 \mathrm{~K}$ is presented and discussed in the second subchapter.

\subsubsection{Multiferroic phase transition}

Between 150 and $20 \mathrm{~K}$, the dielectric permittivity is independent of the frequency and therefore exhibits no Debye-relaxations in the accessible frequency range. The values are different for the three crystallographic directions with $\varepsilon_{\mathrm{r}}^{\prime}(\boldsymbol{a})=21.7 \pm 0.1, \quad \varepsilon_{\mathrm{r}}^{\prime}(\boldsymbol{b})=26.5 \pm 0.1$ and $\varepsilon_{\mathrm{r}}^{\prime}(\boldsymbol{c})=17.8 \pm 0.1$, but they are constant over the whole temperature range within in the experimental uncertainty. The only exception is a distinct anomaly at $T_{\mathrm{N}}$ when the electric field is applied along the $\boldsymbol{b}$-axis, which is depicted in Figure 6-1 together with the permittivity in $\boldsymbol{a}$-direction as comparison.

This peak is, however, very small with a maximum height of $\Delta \varepsilon_{\mathrm{r}}^{\prime} \approx 0.7$ and therefore hard to detect. A high-quality single crystal is necessary, because a broadening of the signal would quickly decrease $\Delta \varepsilon_{r}^{\prime}$ until it is smaller than the experimental error of \pm 0.1 . Misaligned domains or twinning of the crystals can further decrease the signal. Since the peak has a half width of less than $0.5 \mathrm{~K}$, a precise temperature controlling is necessary to observe the signal. The data of Figure 6-1 correspond to a cooling rate of $0.2 \mathrm{~K} \mathrm{~min}^{-1}$. Measurements with higher rates indicated that the peak height will quickly decrease when the temperature is changed too fast.

There are four other studies that measured the dielectric permittivity of BMO at low temperatures [108-110,126]. Golovenchits determined $\varepsilon_{\mathrm{r}}^{\prime}(\boldsymbol{c}) \approx 31$ while Yin found that $\varepsilon_{\mathrm{r}}^{\prime}(\boldsymbol{b}) \approx 31$ and $\varepsilon_{\mathrm{r}}^{\prime}(\boldsymbol{c}) \approx 35$. These values are not only significantly higher than the results of the present thesis, but also the finding that $\varepsilon_{\mathrm{r}}^{\prime}(\boldsymbol{b})>\varepsilon_{\mathrm{r}}^{\prime}(\boldsymbol{c})$ is not supported by Yin. In contrast, the measurement of a ceramic sample by Fier yielded $\varepsilon_{\mathrm{r}}^{\prime} \approx 16$, which is smaller than the average value $\varepsilon_{\mathrm{r}}^{\prime}=22.0$ of the present results. This is not unusual since a ceramic sample that is synthesized by sintering of powder may contain a significant amount of air, which will decrease the capacity of the sample and therefore the determined permittivity. 
The anomaly at $T_{\mathrm{N}}$ was found in none of the four publications. This can be easily explained for the ceramic sample of Fier, since the peak would not only be broadened but also intrinsically smaller due to the averaging over all grain directions. The single crystal data of Golovenchits and Yin, however, only exhibit a small step-like anomaly at $T_{\mathrm{N}}$ but no peak. Since both publications do not provide any information about the crystal quality and the temperature controlling, it remains speculation whether one of these reasons can explain the absence of any peak at the multiferroic phase transition.

Nevertheless, the observation of a peak-like anomaly at $T_{\mathrm{N}}$ during this thesis is clear evidence that BMO is indeed multiferroic. The fact that ferroelectricity exists only along the $\boldsymbol{b}$-axis is consistent with other members of the $\mathrm{R}_{2} \mathrm{Mn}_{4} \mathrm{O}_{10}$-group. The maximum of the peak in Figure 6-1 is located at $T_{\mathrm{C}}=38.3 \mathrm{~K}$ which is close to $T_{\mathrm{N}}$. Since the published values of $T_{\mathrm{N}}$ vary over a few $\mathrm{K}$, depending on sample and method, it is fair to assume that $T_{\mathrm{C}}=T_{\mathrm{N}}$. This suggests that BMO is a type-II multiferroic, which is in accordance with the multiferroic coupling mechanism that was suggested by Chapon.

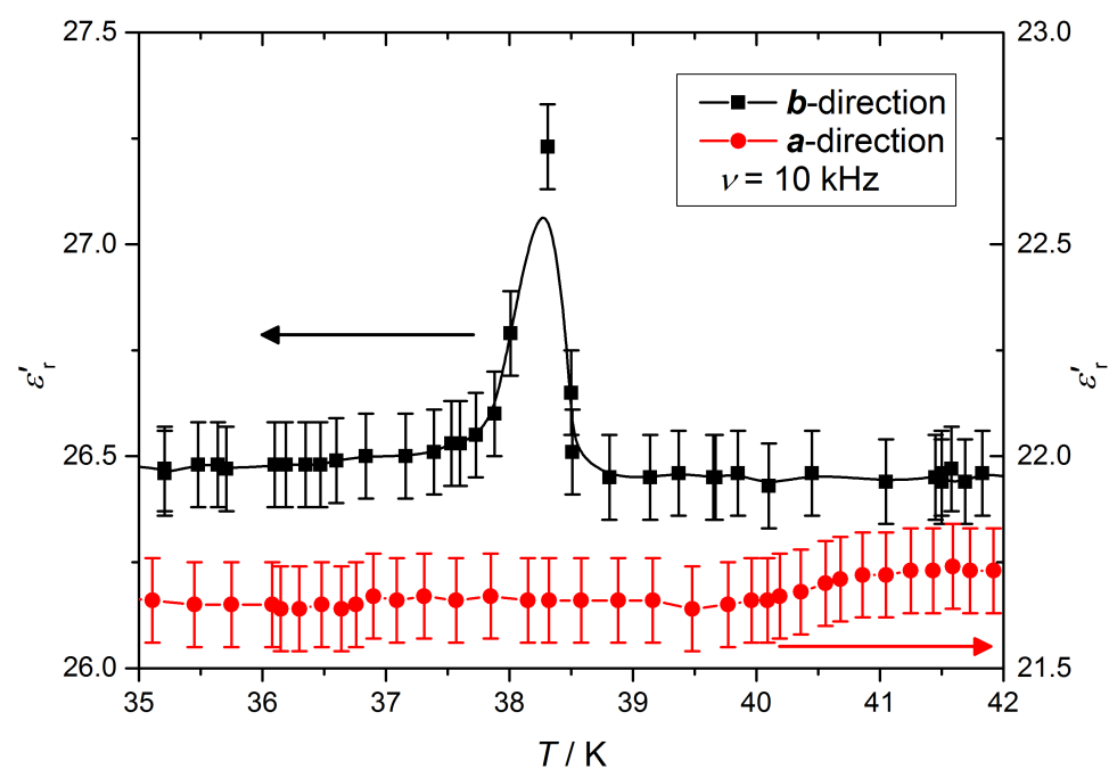

Figure 6-1: Relative permittivity along the $\boldsymbol{b}$-direction (black squares, left axis) and $\boldsymbol{a}$-direction (red circles, right axis) at a frequency of $10 \mathrm{kHz}$ around $T_{\mathrm{N}}$. The cooling rate is $0.2 \mathrm{~K} \mathrm{~min}^{-1}$. Lines are guides to the eye. 


\subsubsection{Relaxation behavior}

Between 300 and $150 \mathrm{~K}$, two Debye-relaxations are observed along each crystallographic axis. The temperature dependence of the real and imaginary part of the dielectric permittivity is presented in Figure 6-2 for a variety of different frequencies. There are two step-like increases of $\varepsilon^{\prime}$ in every direction although the characteristic temperatures and amplitudes are clearly anisotropic. The highest permittivity is observed along the $c$-axis at $T=300 \mathrm{~K}$ with $\mathcal{E}_{\mathrm{r}}^{\prime}(c)=26,000$ followed by $\varepsilon_{\mathrm{r}}^{\prime}(\boldsymbol{a})=20,000$ and $\varepsilon_{\mathrm{r}}^{\prime}(\boldsymbol{b})=14,000$.
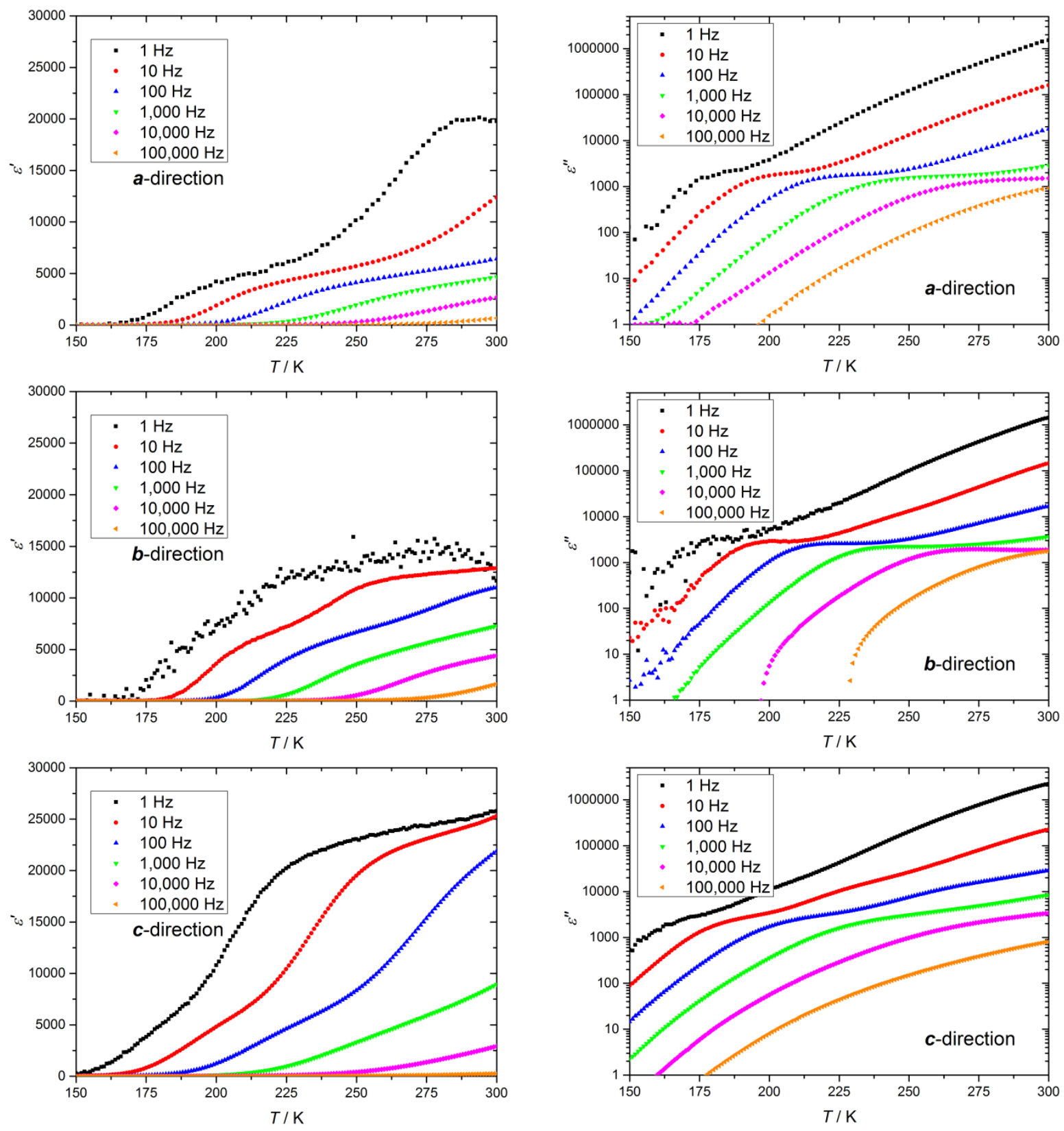

Figure 6-2: Temperature dependence of the real part (left panels) and imaginary part (right panels) of the dielectric permittivity along the $\boldsymbol{a}$ - (top panels), $\boldsymbol{b}$ - (middle panels) and $\boldsymbol{c}$-axis (bottom panels) for different frequencies. Please note, that the imaginary part is plotted logarithmically. 
A function with two Debye-relaxations and a contribution to the imaginary part due to dc-conductivity, according to equations (6-1) and (6-2), was fitted to the spectrum of each temperature which is depicted in Figure 6-3 for an intermediate temperature of $T=250.3 \mathrm{~K}$. Blue and red lines are the contributions of the individual relaxations while the green line corresponds to the dc-conductivity.
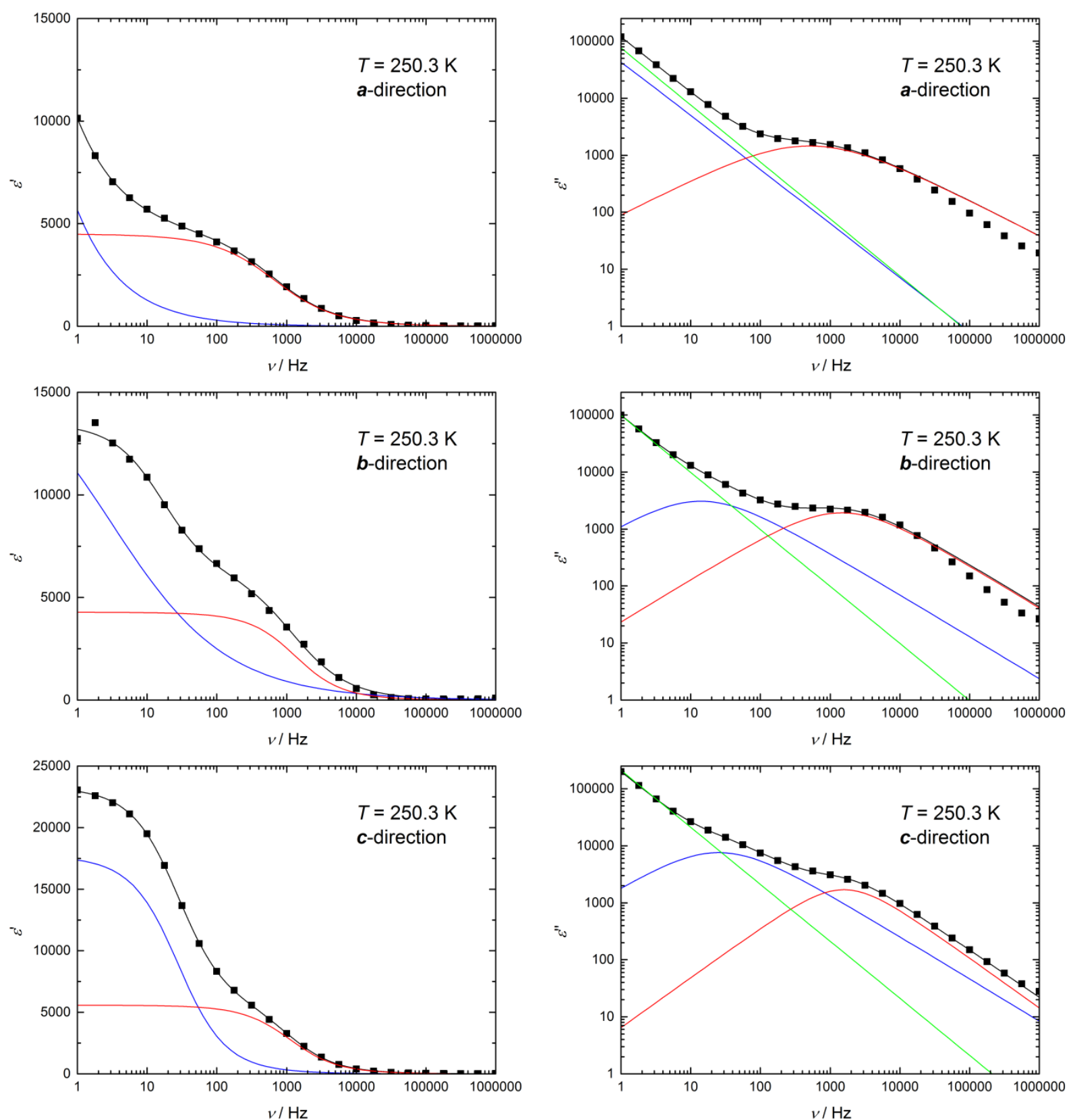

Figure 6-3: Frequency dependence of the real part (left panels) and imaginary part (right panels) of the dielectric permittivity along the $\boldsymbol{a}$ - (top panels), $\boldsymbol{b}$ - (middle panels) and $\boldsymbol{c}$-axis (bottom panels) for $\boldsymbol{a}$ temperature of $T=250.3 \mathrm{~K}$. Black lines are fitted functions according to equations (6-1) and (6-2). Red and blue lines are the contributions of the first and second Debye-relaxation, respectively. The green line corresponds to the dc-conductivity. Please note, that the frequency as well as the imaginary part is plotted logarithmically. 


$$
\begin{gathered}
\varepsilon^{\prime}(\omega)=\varepsilon_{\infty}+\Delta \varepsilon_{1} \frac{1+\cos \left(\beta \frac{\pi}{2}\right)\left(\omega \tau_{1}\right)^{\beta_{1}}}{1+\left(\omega \tau_{1}\right)^{2 \beta_{1}}+2 \cos \left(\beta_{1} \frac{\pi}{2}\right)(\omega \tau)^{\beta_{1}}}+ \\
+\Delta \varepsilon_{2} \frac{1+\cos \left(\beta_{2} \frac{\pi}{2}\right)\left(\omega \tau_{2}\right)^{\beta_{2}}}{1+\left(\omega \tau_{2}\right)^{2 \beta_{2}}+2 \cos \left(\beta_{2} \frac{\pi}{2}\right)\left(\omega \tau_{2}\right)^{\beta_{2}}} \\
\varepsilon^{\prime \prime}(\omega)=-\Delta \varepsilon_{1} \frac{\sin \left(\beta_{1} \frac{\pi}{2}\right)(\omega \tau)^{\beta_{1}}}{1+\left(\omega \tau_{1}\right)^{2 \beta_{1}}+2 \cos \left(\beta_{1} \frac{\pi}{2}\right)\left(\omega \tau_{1}\right)^{\beta_{1}}}- \\
-\Delta \varepsilon_{2} \frac{\sin \left(\beta_{2} \frac{\pi}{2}\right)\left(\omega \tau_{2}\right)^{\beta_{2}}}{1+\left(\omega \tau_{2}\right)^{2 \beta_{2}}+2 \cos \left(\beta_{2} \frac{\pi}{2}\right)\left(\omega \tau_{2}\right)^{\beta_{2}}}-\frac{\sigma}{\varepsilon_{0} \omega}
\end{gathered}
$$

The relaxation times $\tau_{1}$ and $\tau_{2}$ as well as the dc-conductivity $\sigma$ can be extracted from these fits for every temperature. If the relaxations are thermally activated, Arrhenius behavior is expected according to:

$$
\tau=\tau_{0} \exp \left(-\frac{E_{\mathrm{a}}}{k_{\mathrm{b}} T}\right)
$$

$E_{\mathrm{a}}$ is the activation energy that the dipoles have to overcome to reverse their orientation and $\tau_{0}$ is a prefactor that corresponds to the relaxation time at infinite temperatures. The logarithmic relaxation time $\ln (\tau)$ is plotted against the inverse temperature $T^{1}$ in the left panel of Figure 6-4. All relaxations indeed form a straight line and the slope and intercept of a linear fit allows the determination of $E_{\mathrm{a}}$ and $\tau_{0}$, respectively. A similar fit of the temperature corrected dc-conductivity $\sigma T$ is presented in the right panel of Figure 6-4. There is a clear anisotropy in c-direction where the
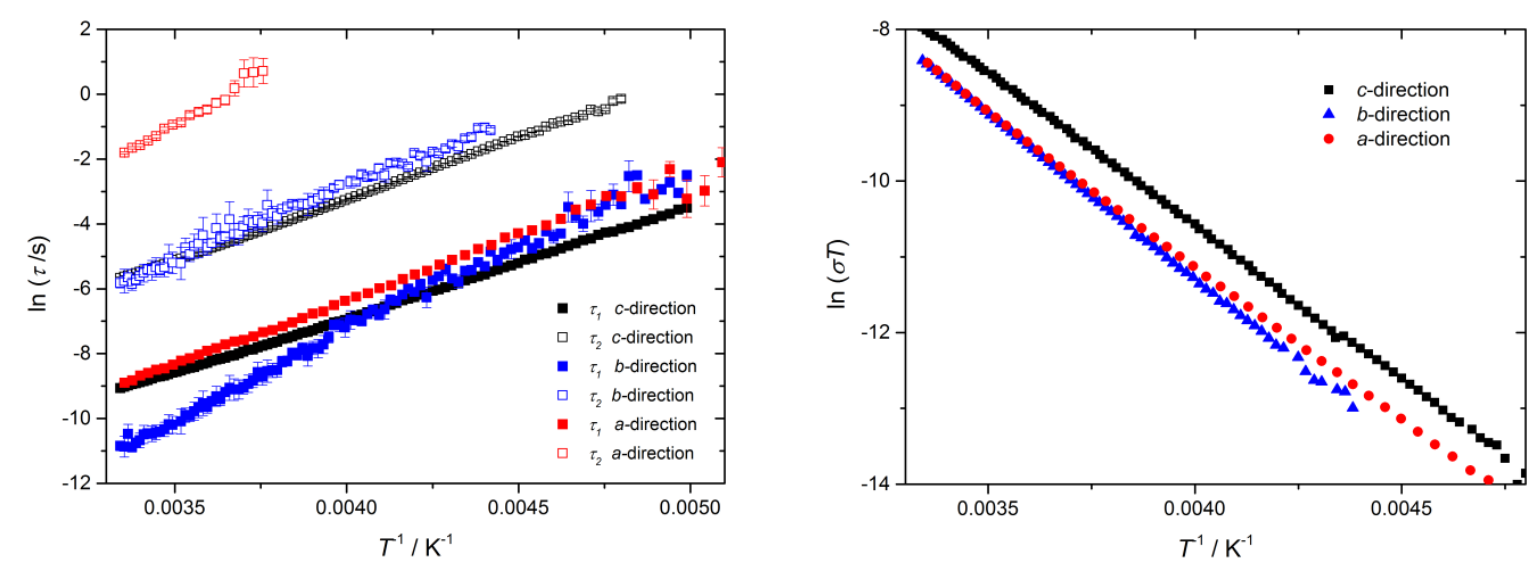

Figure 6-4: Arrhenius-plots of the relaxation times $\tau_{1}$ and $\tau_{2}$ (left) and of the dc-conductivity $\sigma T$ (right) along all three crystallographic directions. 
conductivity is one order of magnitude higher than along the $\boldsymbol{a}$ - and $\boldsymbol{b}$-axis.

All results of the Arrhenius plots are listed in Table 6-2 together with data from literature. The activation energy of the dc-conductivity is nearly identical for all three directions and ranges from 0.35 to $0.38 \mathrm{eV}$. There are two relaxations, one in $\boldsymbol{a}$ - and one in $\boldsymbol{b}$-direction, which have a similar activation energy, but their prefactors of $\tau_{0}=4 \cdot 10^{-11}$ and $\tau_{0}=3 \cdot 10^{-10}$ vary over one order of magnitude. The activation energies of both relaxations in $c$-direction are only slightly smaller, but one of them has a prefactor of $\tau_{0}=2 \cdot 10^{-9}$, which is even one order of magnitude higher. In fact, this is the slowest of all observed relaxations. The highest activation energy is observed for the second relaxation in $\boldsymbol{a}$-direction with $E_{\mathrm{a}}=0.51 \mathrm{eV}$, but the prefactor of $\tau_{0}=6 \cdot 10^{-11}$ is quite similar to the previous relaxations. This is in contrast to another relaxation with increased activation energy of $E_{\mathrm{a}}=0.46 \mathrm{eV}$, which takes place along the $\boldsymbol{b}$-axis and is three orders of magnitude faster with $\tau_{0}=5 \cdot 10^{-14}$. In conclusion, the results of the $c$-axis stand out because not only the dc-conductivity is

Table 6-2: Results of the Arrhenius-plots of Figure 6-4 and comparison with published results in chronological order. The first column denotes the source of the data. The second column provides the information which crystallographic direction can be assigned to the data or if the sample is a ceramic. The activation energies of the relaxations and of the dc-conductivities are listed in the third column, while the fourth column either presents the Arrhenius-prefactor or denotes that the energy of the previous column stems from conductivity measurements.

\section{Annotations:}

${ }^{1}$ : This low-temperature relaxation was not observed in the present experiment.

${ }^{2}$ : This relaxation takes place at temperatures above the temperature range of the present experiment.

${ }^{3}$ : The secondary Arrhenius-behavior below $250 \mathrm{~K}$ was not observed in the present experiment.

${ }^{4}$ : This low-temperature relaxation was not observed in the present experiment.

\begin{tabular}{|c|c|c|c|}
\hline 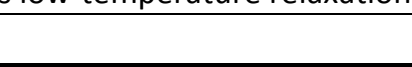 & & $E_{\mathrm{a}} / \mathrm{eV}$ & $\tau_{0} / \mathrm{s}$ \\
\hline \multirow[t]{9}{*}{ this thesis } & \multirow[t]{3}{*}{$\boldsymbol{a}$-direction } & 0.51 & $6 \cdot 10^{-11}$ \\
\hline & & 0.34 & $4 \cdot 10^{-11}$ \\
\hline & & 0.35 & conductivity \\
\hline & \multirow[t]{3}{*}{$\boldsymbol{b}$-direction } & 0.38 & $3 \cdot 10^{-10}$ \\
\hline & & 0.46 & $5 \cdot 10^{-14}$ \\
\hline & & 0.38 & conductivity \\
\hline & \multirow[t]{3}{*}{$c$-direction } & 0.33 & $1 \cdot 10^{-9}$ \\
\hline & & 0.30 & $2 \cdot 10^{-10}$ \\
\hline & & 0.35 & conductivity \\
\hline Golovenchits 1997 [108] & $c$-direction & $0.17^{1}$ & $10^{-11}$ \\
\hline Sun $2006[127]$ & ceramic & 0.37 & $5 \cdot 10^{-14}$ \\
\hline \multirow[t]{3}{*}{ Lin 2009 [128] } & \multirow[t]{3}{*}{ ceramic } & $0.18^{1}$ & $5 \cdot 10^{-11}$ \\
\hline & & 0.38 & $2 \cdot 10^{-11}$ \\
\hline & & 0.37 & conductivity \\
\hline Fier 2011 [126] & ceramic & $1.24^{2}$ & $5 \cdot 10^{-15}$ \\
\hline \multirow[t]{2}{*}{ Fier 2012 [109] } & \multirow[t]{2}{*}{ ceramic } & $0.0492^{3}$ & conductivity \\
\hline & & 0.48 & conductivity \\
\hline \multirow[t]{4}{*}{ Yin 2013 [110] } & $\boldsymbol{b}$-direction & $0.065^{4}$ & $3 \cdot 10^{-10}$ \\
\hline & $\boldsymbol{b}$ - and $\boldsymbol{c}$-direction & $0.203^{1}$ & $1 \cdot 10^{-12}$ \\
\hline & $\boldsymbol{b}$ - and $\boldsymbol{c}$-direction & 0.298 & $5 \cdot 10^{-10}$ \\
\hline & $\boldsymbol{b}$-direction & 0.225 & conductivity \\
\hline
\end{tabular}


one order of magnitude higher in this direction, but also both relaxations have a lower activation energy and a higher prefactor than all other observed relaxations.

Furthermore, Table 6-2 lists all available activation energies and prefactors that are provided by different publications about the dielectric behavior of BMO. The relaxation along the $c$-axis that is presented by Golovenchits takes places at temperatures slightly below $150 \mathrm{~K}$ where no relaxation is observed in the present experiments. In contrast, the relaxation of Fier 2011 stems from measurements at much much higher temperatures, which are beyond the scope of this thesis. Consequently, the values of both publications cannot be compared to the results of the present study.

The activation energies of the dc-conductivity are similar to the results of this thesis and range from 0.225 to $0.48 \mathrm{eV}$. The only exception is the second contribution to the conductivity that is observed by Fier 2012 below $250 \mathrm{~K}$ and that has an activation energy of $E_{\mathrm{a}}=0.0492 \mathrm{eV}$.

Sun and Lin used ceramic samples for dielectric measurements, which makes it difficult to compare their results with the findings of this thesis, because relaxations along all three crystallographic axes contribute to the measured signal. This may explain why both found only one relaxation between 300 and $150 \mathrm{~K}$. They have a nominal activation energy that is consistent with the present results although Sun's prefactor is several orders of magnitude smaller. Yin, on the other hand, presents single crystal data and also found only one relaxation in this temperature range. The absence of the second relaxation therefore may have another reason than the polycrystallinity of the ceramic sample. Yin, however, determined this relaxation along the $\boldsymbol{b}$-and $\boldsymbol{c}$-axis and found that it is identical in both directions, which contradicts the anisotropy that is found in the present experiment. Lin and Yin furthermore observed the relaxation below $150 \mathrm{~K}$ that was first presented by Golovenchits. Yin even found a third relaxation at low temperatures slightly above $T_{\mathrm{N}}$, which is absent in all other studies.

In summary, the results of the dielectric relaxations strongly depend on the individual sample. In this thesis, two relaxations are found for each crystallographic axis between 300 and $150 \mathrm{~K}$. All other sources observed in this temperature range only one relaxation, which at least has a similar activation energy. The obvious anisotropy of the present results is in disagreement with Yin. There are reports about two other relaxations at temperatures below $150 \mathrm{~K}$ that are not observed in this thesis.

The only common ground of all studies are the relaxations with an activation energy of $0.3-0.4 \mathrm{eV}$. There is a broad agreement that this relaxation is a polaron induced by oxygen vacancies $[110,127,128]$. Thermally activated oxidation of these vacancies leads to free charges that are 
coupled to the lattice and create localized dipoles due to a distortion of their surroundings. These dipoles are believed to be the basis for the Debye-relaxation and the oxidation energy corresponds to the activation energy $E_{\mathrm{a}}$. The charges contribute to the dc-conductivity, which explains why the activation energies of the conductivity and of the relaxations are so similar. This kind of relaxation is also observed in other substances like for example perovskites $[129,130]$. However, this also means that the relaxations strongly depend on the number of defects and, consequently, on the general sample quality. The additional relaxation below $150 \mathrm{~K}$ that is observed by Golovenchits, Lin and Yin might therefore be a sign for an increased amount of defects in their sample, which can also explain the absence of a peak-like anomaly of the dielectric permittivity at $T_{N}$, as discussed in the previous chapter.

\subsubsection{Amendment after submission of the thesis}

After the submission of this thesis, the author became aware of an article by Khannanov et al. [131], which was published in the year 2017 and examines the dielectric permittivity, electric polarization and conductivity of $\mathrm{Bi}_{2} \mathrm{Mn}_{4} \mathrm{O}_{10}$ and $\mathrm{Gd}_{2} \mathrm{Mn}_{4} \mathrm{O}_{10}$ crystals below room temperature. There is a slight maximum of $\varepsilon^{\prime}$ measured along the $\boldsymbol{b}$-direction of $\mathrm{BMO}$ at the magnetic phase transition temperature $T_{N}$, which is in accordance with the results of Figure $6-1$. The anomaly observed by Khannanov is, however, considerably broader and less pronounced, which might be either the result of a higher cooling rate during the measurement or an indication that the crystal has more defects than the present sample. Furthermore, thermo-stimulated pyrocurrent measurements clearly show the onset of electric polarization at $T_{\mathrm{N}}$ and a further increase at lower temperatures, which correlates with the magnetic ordering. Both results are evidence for type-II multiferroicity in BMO. It should be emphasized that the article of Khannanov was published a year before the submission of this thesis. The implicit claim that the results of Figure 6-1 are the first evidence for multiferroicity of BMO must therefore be withdrawn by the author of the thesis.

Measurements of the dielectric permittivity by Khannanov furthermore reveal two relaxation processes along all three crystallographic directions well above $T_{\mathrm{N}}$, which is in qualitative agreement with the results of the present thesis. The activation energies that are determined by the temperature dependence of the relaxations significantly differ from the results presented in Table 6-2. However, those values might not be too accurate due to the limited frequency range, which covers less than two orders of magnitude. 


\subsection{Determination of elastic constants}

This chapter will provide an overview over the inelastic neutron scattering measurements that were conducted to determine the elastic constants of BMO in the paramagnetic as well as in the multiferroic phase. The first subchapter explains how sound velocities can in principle be extracted from spectra of acoustic phonons and which practical obstacles need to be overcome. This is combined with a detailed description of the data evaluation. The actual results of the roomtemperature measurements are presented and interpreted in the second subchapter together with an explanation of the mechanical anisotropy in BMO. The effect of the multiferroic phase transition on the acoustic phonons and, consequently, the elastic constants is described in the last subchapter.

\subsubsection{Evaluation of data}

As explained in chapter 3.3 , it is necessary to measure the velocity of a number of different sound waves in order to determine all elastic constants of BMO. Inelastic neutron scattering is a suitable method for this task, since the sound velocities can be extracted from the dispersion relation of acoustic phonons. This is schematically exemplified in Figure 6-5 where the angular frequency $\omega$ of a phonon is plotted against its reduced wave vector $\xi$. At low values of $\xi$, close to the $\Gamma$-point, the frequency is linearly increasing while it approaches a constant value at high $\xi$, close to the Brilliounzone boundary. Sound waves are collective long-wavelength vibrations where all atoms are displaced into the same direction. They can be described as acoustic phonons with a long wavelength and the sound velocity is therefore the group velocity at the $\Gamma$-point:

$$
v_{s}=\left.\frac{\mathrm{d} \omega}{\mathrm{d} q}\right|_{q=0}
$$

This is equal to the slope of the red dashed line in Figure 6-5. Consequently, the measurement strategy is to determine the frequency of longitudinal as well as transverse acoustic phonons for different absolute values of $\boldsymbol{q}$ and in different directions. The sound velocities are then extracted by a linear least-squares fit, allowing the calculation of elastic constants.

Longitudinal and transverse acoustic phonons can be experimentally distinguished due to the polarization selection rule which says that the scattered intensity is proportional to the square of the dot product between scattering vector $\boldsymbol{Q}$ and polarization vector $\boldsymbol{u}$ :

$$
I \propto|Q \cdot u|^{2}
$$

$\boldsymbol{Q}$ itself is the sum of the lattice vector $\boldsymbol{g}$, which determines the Brillouin-zone, and the phonon wave vector $\boldsymbol{q}$. The ideal scattering geometries for longitudinal and transverse phonons are schematically exemplified in Figure $6-6$ for a phonon that is propagating along the $\boldsymbol{a}$-axis. In the 
longitudinal case, $\boldsymbol{u}$ is parallel to $\boldsymbol{q}$ and the maximum intensity is achieved if $\boldsymbol{g}$ has only an $a$-component, because $\boldsymbol{Q}$ and $\boldsymbol{u}$ are then perfectly parallel. For the transverse phonon, on the other hand, it is advisable to choose a Brillouin-zone along $\boldsymbol{b}$, because this will minimize the angle between $\boldsymbol{Q}$ and $\boldsymbol{u}$ as much as possible. In conclusion, the choice of the Brillouin-zone determines which phonons are detected for a given phonon wave vector $\boldsymbol{q}$.

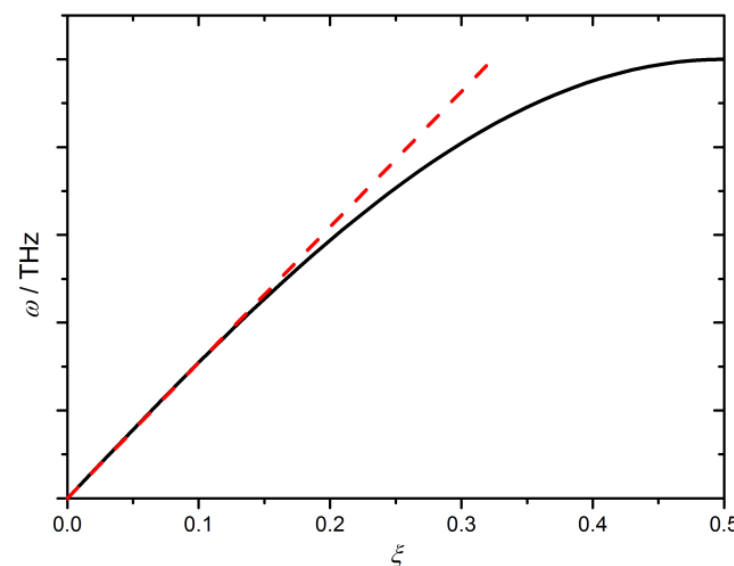

Figure 6-5: Schematic representation of the dispersion curve of an acoustic phonon. The $x$-axis is the reduced phonon wave vector and the $y$-axis is the angular frequency of the phonon. The slope of the red dashed line is equal to the sound velocity.

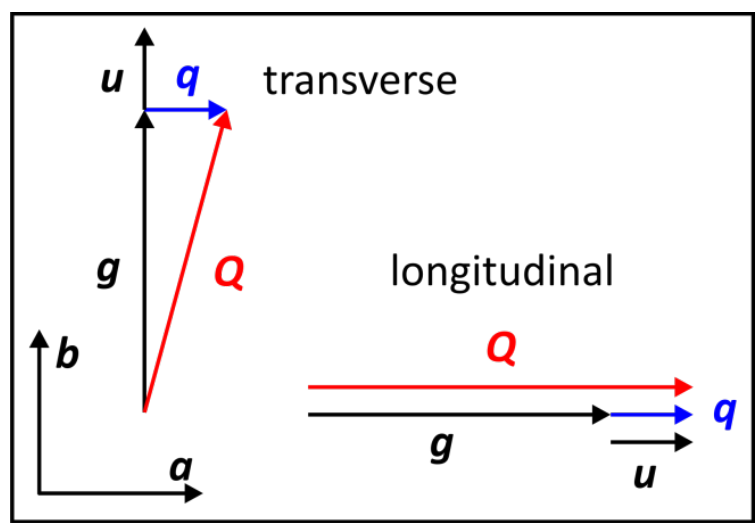

Figure 6-6: Schematic representation of the polarization selection rule for acoustic phonons. $\boldsymbol{Q}$ is the scattering vector and $\boldsymbol{g}$ is a lattice vector while $\boldsymbol{q}$ and $\boldsymbol{u}$ are the propagation and polarization vector, respectively, of the phonon.

The first two panels of Figure 6-7 are examples for the polarization selection rule. The spectrum in the top-left shows three constant- $\boldsymbol{q}$-scans of a transverse phonon that propagates in $\boldsymbol{a}$-direction. Since the $(0,0,4)$-Brillouin zone is chosen, this phonon is polarized in c-direction and called $T_{2} A$, according to the nomenclature of Table 3-3. The scans are at three different points in momentum space with equal distance and the intensity maxima are also at equidistant energies. This illustrates that the dispersion relation of this particular phonon is linear throughout half of the Brillouin-zone. The same is true for the phonon whose spectra are depicted in the top-right panel of Figure 6-7. This is a longitudinal phonon that propagates along the $\boldsymbol{b}$-axis. The ideal choice of Brillionzone for this phonon, according to the polarization selection rule, is $(0,4,0)$, but instead it is measured in the $(1,4,0)$-BZ because the structure factor of this phonon is significantly smaller in the $(0,4,0)$-BZ. These two competing effects have to be carefully considered, for example by using the lattice dynamics simulation package UNISOFT [132]. All acoustic phonons of BMO that are polarized along the $\boldsymbol{b}$-axis have their highest intensity in the $(1,4,0)$-BZ. The Brillouin-zones for the other two polarizations are $(4,0,0)$ and $(0,0,4)$.

The spectra in the top-right panel of Figure 6-7 are constant-energy-scans where the detected momentum transfer is varied. These types of scan are employed for all measurements of longitudinal 
phonons while constant- $\boldsymbol{q}$-scans are generally chosen for transverse phonons. Since the initial slope of the dispersion relation is considerably higher in the longitudinal case, it is necessary to measure closer to the $\Gamma$-point in order to achieve enough data points before the dispersion curve flattens. In this part of the Brillouin-zone, the measured peak profiles of phonons in positive and negative direction of $\boldsymbol{q}$ overlap, due to the finite resolution of the spectrometer. For this reason, collimation is used for all measurements of longitudinal phonons, while the resolution of the focused monochromator is good enough for transverse phonons. The overlapping profiles can be distinguished if two Gauß-functions are fitted to the scan data, as presented in the bottom-left panel of Figure 6-7 for the phonon that propagates in $\boldsymbol{a}$-direction as arbitrary example. Furthermore, this has the fortunate side effect that two data points of the dispersion curve, $\boldsymbol{q}$ and $-\boldsymbol{q}$, can be obtained by a single scan.
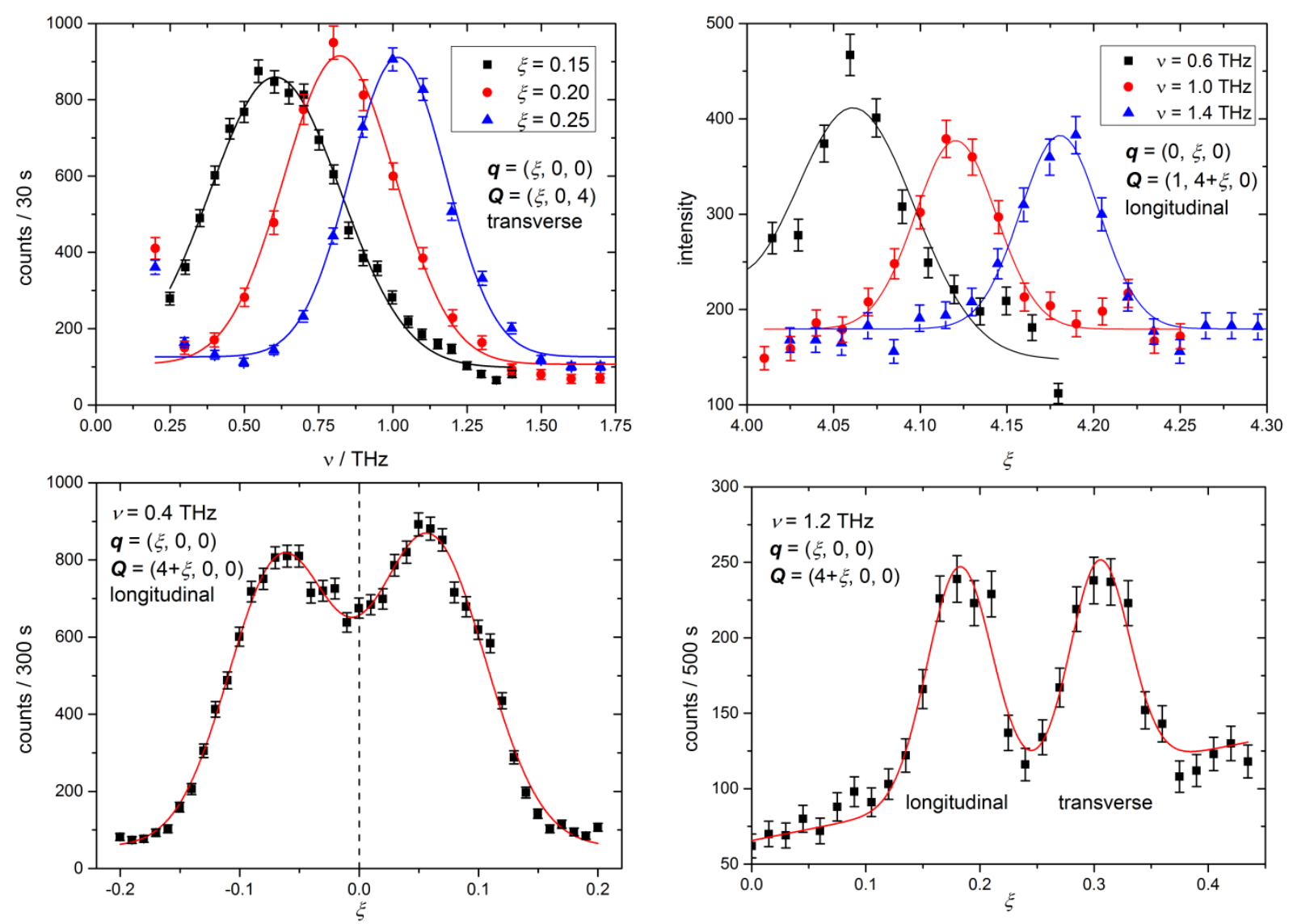

Figure 6-7: (top-left): Constant- $\boldsymbol{q}$-scans of a transverse phonon with different values of $\boldsymbol{q}$. (top-right): Constant-energy-scans of a longitudinal phonon with different energy transfers. (bottom-left): Constantenergy-scan of a longitudinal phonon in positive and negative direction of $\boldsymbol{q}$. (bottom-right): Constantenergy-scan of a longitudinal phonon where a transverse phonon is detected despite the fact that it should have zero intensity according to the polarization selection rules. Lines in all four panels are Gauß-profiles that are determined by a least-squares fit.

There are a number of scans that seemingly violate the polarization selection rule. One example is presented in the bottom-right panel of Figure 6-7, where the longitudinal phonon along 
the $\boldsymbol{a}$-axis is measured and although the transverse phonon should have zero intensity, it is clearly detected. This can also be explained by the finite resolution of the spectrometer, since not only phonons with nominal momentum transfer $\boldsymbol{Q}$ contribute to the intensity of a scan point, but also phonons close in momentum space with $\boldsymbol{Q}+\Delta \boldsymbol{Q}$. The angle between $\boldsymbol{Q}+\Delta \boldsymbol{Q}$ and $\boldsymbol{u}$ might be non-perpendicular and the according phonon can therefore contribute to the scan point. This intensity is much less, compared to the ideal scattering geometry, but if the structure factor is high enough, the "forbidden" phonon might have as much intensity as the "allowed" one, like in the bottom-right panel of Figure 6-7.

Another example is the spectrum presented in Figure 6-8 where three distinct peaks are visible. The pseudo-longitudinal (LA) and pseudo-transverse $\left(T_{1} A\right)$ phonons that propagate in $(0, \xi, \xi)$-direction are both allowed due to the polarization selection rule since the scattering vector $\boldsymbol{Q}$ has a non-perpendicular angle to $\boldsymbol{u}_{\mathrm{LA}}$ as well as to $\boldsymbol{u}_{\mathrm{T} 1 \mathrm{~A}}$. The peak in the middle of the spectrum is assigned to the transverse phonon $\mathrm{T}_{2} \mathrm{~A}$ which is definitely forbidden, since $\boldsymbol{u}_{\mathrm{T} 2 \mathrm{~A}}$ is perpendicular to the scattering plane. This particular spectrum is measured with doubly focused monochromator. Due to the rather large vertical divergence of about $2^{\circ}$, phonons with a propagation vector $\boldsymbol{q}_{\delta}=(\delta, \xi, \xi)$, which is outside of the nominal scattering plane, can contribute to the scattered intensity. In this case, the propagation tensor cannot be divided into a $1 \times 1$ - and $2 \times 2$-block and the rule that $\boldsymbol{u}_{\mathrm{LA}}$ and $\boldsymbol{u}_{\mathrm{TAA}}$ are in the scattering plane while $\boldsymbol{u}_{\mathrm{T} 2 \mathrm{~A}}$ is perpendicular is not true anymore. In fact, calculations with UNISOFT revealed that the polarization vectors of all three phonons change quite rapidly with $\delta$ and so increases the intensity of $T_{2} A$.

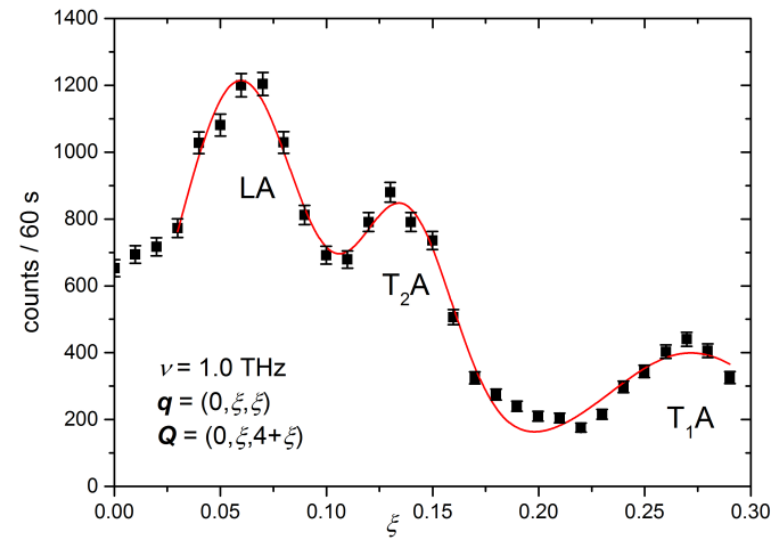

Figure 6-8: Resolution artifact where three phonons are simultaneously detected although $\mathrm{T}_{2} \mathrm{~A}$ should have zero intensity according to the polarization selection rule. The red curve is the sum of three Gauß-profiles that are determined by a least-squares fit.

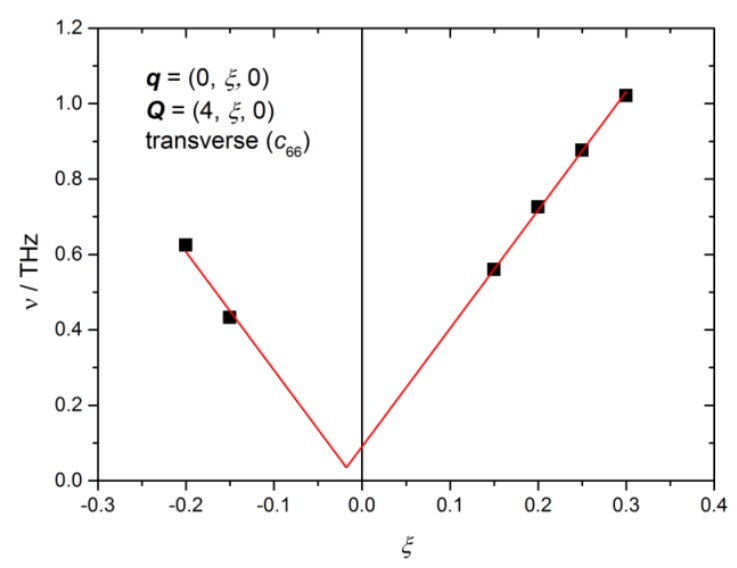

Figure 6-9: Example of the fit function with offset that is used to determine the slope of the dispersion curves with data points in $\boldsymbol{q}$ - and $-\boldsymbol{q}$ direction. 
The frequency of acoustic phonons should be zero at the $\Gamma$-point, but the dispersion relation of many phonons could only be satisfactorily fitted by a linear function if a non-zero frequency was assumed. Otherwise, the slopes obtained from the $+\boldsymbol{q}$ - and $-\boldsymbol{q}$-dispersion curves differed significantly in many cases. The best results are achieved if the data in positive and negative direction are simultaneously considered and an offset in momentum $\left(q_{0}\right)$ and frequency $\left(v_{0}\right)$ is introduced:

$$
v=v_{\mathrm{s}}\left|q-q_{0}\right|+v_{0}
$$

This offset is rather small but it has to be considered in order to determine accurate values of the slope. The origin of this offset may be a slight miscalibration of the spectrometer, which also supported by the fact that the values of $q_{0}$ and $v_{0}$ are similar during one measuring campaign and differ with respect to the next campaign while the instrument was recalibrated in between. An application of this fit function on a transverse phonon propagating along the $\boldsymbol{b}$-axis as arbitrary example is presented in Figure 6-9. The data points of all dispersion curves are corrected by the offset before they are presented in Figure 6-10 and Figure 6-11 in chapter 6.4.2.

There are, however, three phonons whose dispersion relation could not be evaluated by this method. The pseudo-transverse phonons that propagate in $(0, \xi, \xi)$ - and $(\xi, 0, \xi)$-direction and the pseudo-longitudinal phonon that propagates along $(\xi, \xi, 0)$ are only measured along positive $\boldsymbol{q}$. A simple linear fit without any offset is enough for the two pseudo-transverse cases. The pseudolongitudinal phonon, however, is the only phonon where a considerably flattening of the dispersion curve was observed. Therefore, only the first four data points are fitted by a linear function with offset (see Figure 6-11). 


\subsubsection{Room temperature results}

This chapter focusses on the results obtained at room temperature and also provides an interpretation of the anisotropy of the elastic constants. The temperature-dependence of the acoustic phonons and especially the effect of the multiferroic phase transition is subject of the next chapter.

The dispersion relation of all measured phonons that propagate along the principal axes are presented in Figure 6-10. Although it is sufficient to determine the sound velocities of only three transverse phonons in order to obtain all elastic constants, redundant measurements like $T_{2} A$ with $\boldsymbol{q}=(\xi, 0,0)$ and $\mathrm{T}_{1} \mathrm{~A}$ with $\boldsymbol{q}=(0, \xi, 0)$, which both provide $c_{66}$, are still useful to check for consistency of the results. The transverse phonon $\mathrm{T}_{2} \mathrm{~A}$ with $\boldsymbol{q}=(0,0, \xi)$ was, however, not accessible because the Brillouin-zone of choice for a phonon that is polarized along the $\boldsymbol{b}$-axis is $(1,4,0)$, resulting in the scattering vector $Q=(1,4, \xi)$ which is outside of all three scattering geometries that are used in this experiment.

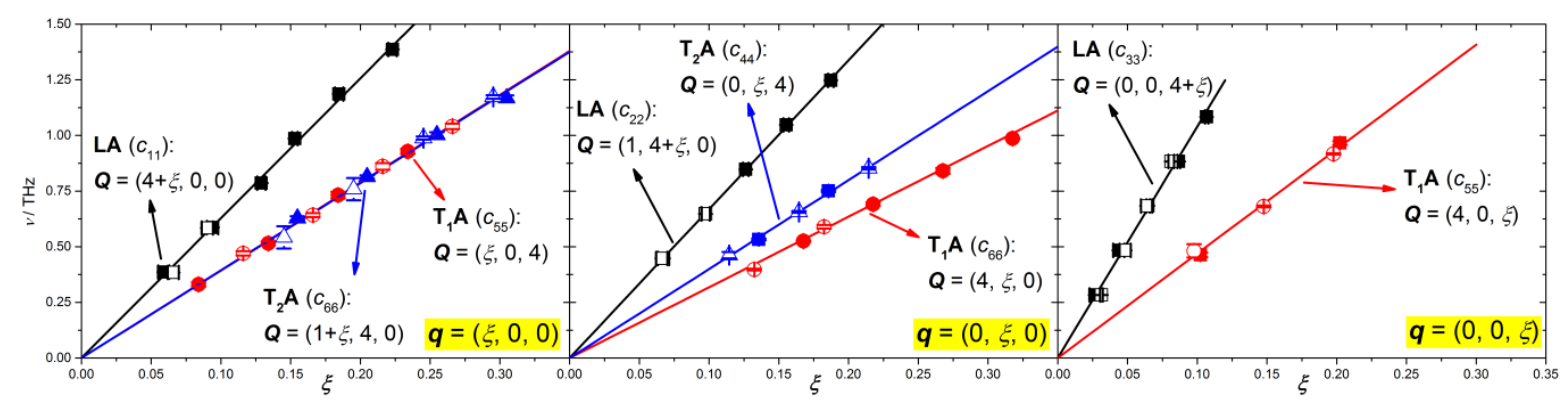

Figure 6-10: Dispersion relation for all measured acoustic phonons with propagation vectors along the principal axes $\boldsymbol{a}, \boldsymbol{b}$, and $\boldsymbol{c}$. Each figure shows a plot of the phonon frequency against the reduced phonon wave vector. Squares are the longitudinal phonon (LA). Circles and triangles are the two transverse phonons $T_{1} A$ and $T_{2} A$, respectively. Full and open symbols represent data obtained for positive and negative values of $\xi$, respectively. The experimental error bars are in general smaller than the symbol size. The labels also display the associated elastic constant and the scattering vector $\boldsymbol{Q}$. Solid lines represent the fits which were used to calculate the sound velocity.

Table 6-3: Sound velocity (third column) and eigenvalue (fourth column) for acoustic phonons propagating along the principal directions. The last column denotes the corresponding elastic constant.

\begin{tabular}{|c|c|c|c|c|}
\hline & & $v_{\mathrm{s}} / \mathrm{m} \mathrm{s}^{-1}$ & $\rho v_{\mathrm{s}}^{2} / \mathrm{GPa}$ & \\
\hline \multirow{3}{*}{$(\xi, 0,0)$} & $\mathrm{LA}$ & $4740 \pm 240$ & $160 \pm 16$ & $c_{11}$ \\
\cline { 2 - 5 } & $\mathrm{T}_{1} \mathrm{~A}$ & $2970 \pm 150$ & $63 \pm 7$ & $c_{55}$ \\
\cline { 2 - 5 } & $\mathrm{T}_{2} \mathrm{~A}$ & $2960 \pm 150$ & $63 \pm 7$ & $c_{66}$ \\
\hline \multirow{3}{*}{$(0, \xi, 0)$} & $\mathrm{LA}$ & $5710 \pm 290$ & $233 \pm 24$ & $c_{22}$ \\
\cline { 2 - 5 } & $\mathrm{T}_{1} \mathrm{~A}$ & $3010 \pm 150$ & $51 \pm 6$ & $c_{66}$ \\
\cline { 2 - 5 } & $\mathrm{T}_{2} \mathrm{~A}$ & $3410 \pm 170$ & $83 \pm 9$ & $c_{44}$ \\
\hline \multirow{2}{*}{$(0,0, \xi)$} & $\mathrm{LA}$ & $5990 \pm 300$ & $257 \pm 26$ & $c_{33}$ \\
\cline { 2 - 5 } & $\mathrm{T}_{1} \mathrm{~A}$ & $2710 \pm 150$ & $52 \pm 6$ & $c_{55}$ \\
\hline
\end{tabular}


The resulting sound velocities, eigenvalues of the propagation tensor and the corresponding elastic constants are listed in Table 6-3. The two redundant constants $c_{55}$ and $c_{66}$ differ by approximately $20 \%$, which is in accordance with a relative error of $10 \%$ that is estimated for all eigenvalues. The weighted average is used to calculate the final results, which are listed in Table 6-5.

The dispersion relation for phonons that propagate in between two principal axes are presented in Figure 6-11 and the resulting sound velocities and eigenvalues are listed in Table 6-4. The eigenvalue of the transverse phonon $\mathrm{T}_{2} \mathrm{~A}$ that propagates along $\boldsymbol{q}=(0, \xi, \xi)$ is a linear combination of $c_{55}$ and $c_{66}$ (see Table 3-4) and can therefore be calculated from the results of the principal axes. Due to the experimental error of $10 \%$, the confidence intervals of the calculated and the experimentally determined eigenvalues sligthly overlap. This further confirms the assumptions that this signal is a result of the $T_{2} A$ phonon.

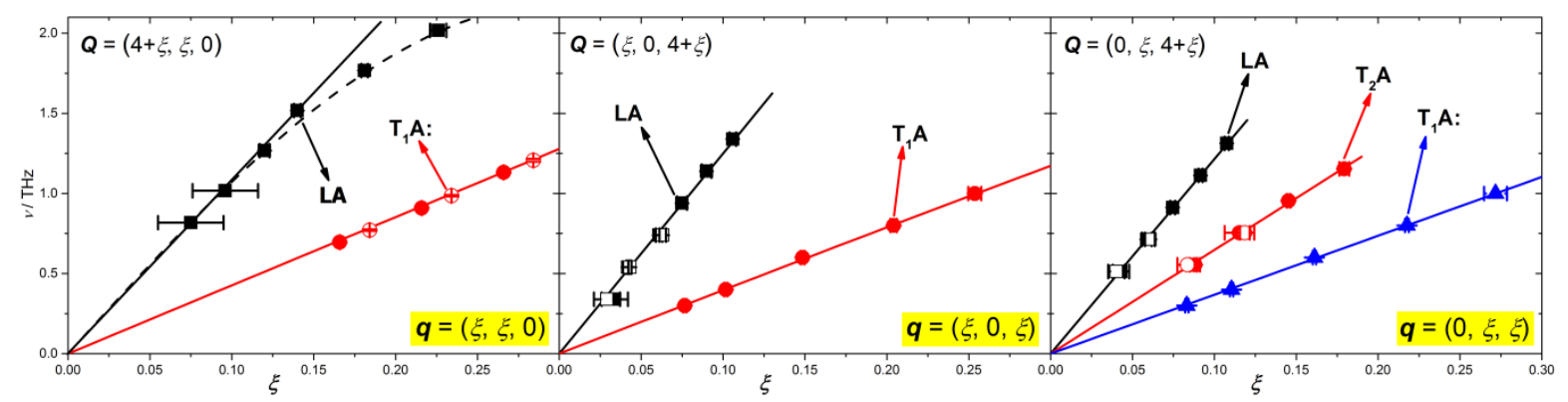

Figure 6-11: Dispersion relation for all measured acoustic phonons with propagation vectors along $(\xi, \xi, 0)$, $(\xi, 0, \xi)$ and $(0, \xi, \xi)$. Each figure shows a plot of the phonon frequency against the reduced phonon wave vector. Squares are the longitudinal phonon (LA). Circles and triangles are the two transverse phonons $T_{1} A$ and $T_{2} A$, respectively. Full and open symbols represent data obtained for positive and negative values of $\xi$, respectively. The experimental error bars are in general smaller than the symbol size. Solid lines represent the fits which were used to calculate the velocity of sound. All phonons with the same propagation vector were measured in the same Brillouin-zone as indicated by the scattering vector $\boldsymbol{Q}$ in each figure.

Table 6-4: Sound velocity (third column) and eigenvalue (fourth column) for acoustic phonons with propagation vectors in between the principal axes.

\begin{tabular}{|c|c|c|c|}
\hline & & $v_{\mathrm{s}} / \mathrm{m} \mathrm{s}^{-1}$ & $\rho v_{\mathrm{s}}{ }^{2} / \mathrm{GPa}$ \\
\hline \multirow{2}{*}{$(\xi, \xi, 0)$} & $\mathrm{LA}$ & $6060 \pm 300$ & $262 \pm 27$ \\
\cline { 2 - 4 } & $\mathrm{T}_{1} \mathrm{~A}$ & $2430 \pm 120$ & $42 \pm 5$ \\
\hline \multirow{2}{*}{$(\xi, 0, \xi)$} & $\mathrm{LA}$ & $5720 \pm 290$ & $234 \pm 24$ \\
\cline { 2 - 4 } & $\mathrm{T}_{1} \mathrm{~A}$ & $1810 \pm 90$ & $23 \pm 3$ \\
\hline \multirow{3}{*}{$(0, \xi, \xi)$} & $\mathrm{LA}$ & $5810 \pm 290$ & $241 \pm 25$ \\
\cline { 2 - 4 } & $\mathrm{T}_{1} \mathrm{~A}$ & $1760 \pm 90$ & $22 \pm 3$ \\
\cline { 2 - 4 } & $\mathrm{T}_{2} \mathrm{~A}$ & $3090 \pm 160$ & $68 \pm 7$ \\
\hline
\end{tabular}


Equations (3-43), (3-45) and (3-47) allow the calculation of the elastic off-diagonal elements. Since the diagonal elastic constants are known from the measurements along the principal axes, these equations have only one unknown parameter which can be obtained on three ways: with the eigenvalue of the pseudo-longitudinal phonon, with the eigenvalue of the pseudo-transverse phonon or with the difference of both eigenvalues according to the following equation (exemplified for $\Gamma(\xi, \xi, 0))$ :

$$
\rho v_{s, \mathrm{LA}}^{2}-\rho v_{s, \mathrm{~T}_{1} \mathrm{~A}}^{2}=\frac{2}{1+\gamma^{2}} \sqrt{\gamma^{2}\left(c_{12}+c_{66}\right)^{2}+1 / 4\left(c_{11}-c_{66}+\gamma^{2}\left(c_{66}-c_{22}\right)\right)^{2}}
$$

In fact, all three ways lead to slightly different results and therefore the weighted averages are listed in Table 6-5.

Table 6-5: List of all elastic constants.

\begin{tabular}{|c|c|c|c|c|c|}
\hline $\mathrm{ij}$ & $c_{\mathrm{ij}} / \mathrm{GPa}$ & $\mathrm{ij}$ & $c_{\mathrm{ij}} / \mathrm{GPa}$ & $\mathrm{ij}$ & $c_{\mathrm{ij}} / \mathrm{GPa}$ \\
\hline 11 & $160 \pm 16$ & 44 & $83 \pm 9$ & 12 & $134 \pm 14$ \\
\hline 22 & $233 \pm 24$ & 55 & $56 \pm 6$ & 13 & $157 \pm 16$ \\
\hline 33 & $257 \pm 26$ & 66 & $58 \pm 6$ & 23 & $187 \pm 19$ \\
\hline
\end{tabular}

The final results of all nine elastic constants are presented in Table 6-5. The only published quantity for a comparison is the bulk modulus $B$, which can be calculated from the elastic constants according to the following relation, which is valid for orthorhombic symmetry:

$$
B=\frac{c_{11} c_{22} c_{33}-c_{13}{ }^{2} c_{22}-c_{12}{ }^{2} c_{33}+2 c_{12} c_{13} c_{23}-c_{11} c_{23}{ }^{2}}{\left(c_{22}+c_{11}-2 c_{12}\right) c_{33}+\left(c_{11}-2 c_{13}\right) c_{22}-c_{23}{ }^{2}-2\left(c_{11}-c_{12}-c_{13}\right) c_{23}-\left(c_{12}-c_{13}\right)^{2}}
$$

The result of $B=154 \pm 14 \mathrm{GPa}$ is slightly larger than the published value of $B=138 \pm 1 \mathrm{GPa}$, which was obtained by pressure dependent powder X-ray diffraction [98], but the confidence intervals of both results nearly overlap.

Furthermore, the elastic constants allow the calculation of the polarization vectors $\boldsymbol{u}$ of the pseudo-longitudinal and pseudo-transverse acoustic phonons that propagate in between the principal directions. They are listed in Table 6-6 together with the angle $\alpha$ between $\boldsymbol{q}$ and $\boldsymbol{u}_{\mathrm{LA}}$, which varies between $2.7^{\circ}$ and $6.4^{\circ}$. Since $\alpha$ is rather small, it is justified to use the expressions "pseudolongitudinal" and "pseudo-transverse" that are introduced in chapter 3.3. 
Table 6-6: Polarization vector $\boldsymbol{u}$ for the pseudo-longitudinal (LA) (third column) and pseudo-transverse $\left(\mathrm{T}_{1} \mathrm{~A}\right)$ (fourth column) acoustic phonon for different propagation vectors $\boldsymbol{q}$. The angle $\alpha$ between $\boldsymbol{q}$ and $\boldsymbol{u}_{\mathrm{LA}}$ are listed in the fifth column.

\begin{tabular}{|c|c|c|c|c|}
\hline \multicolumn{2}{|c|}{} & \multicolumn{2}{|c|}{$\mathbf{u} / \AA^{-1}$} & $\alpha /^{\circ}$ \\
\hline r.l.u. & $\AA^{-1}$ & LA & $\mathrm{T}_{1} \mathrm{~A}$ & \\
\hline$\left(\begin{array}{l}1 \\
1 \\
0\end{array}\right)$ & $\left(\begin{array}{c}0.749 \\
0.662 \\
0\end{array}\right)$ & $\left(\begin{array}{c}0.671 \\
0.742 \\
0\end{array}\right)$ & $\left(\begin{array}{c}-0.742 \\
0.671 \\
0\end{array}\right)$ & 6.4 \\
\hline$\left(\begin{array}{l}1 \\
0 \\
1\end{array}\right)$ & $\left(\begin{array}{c}0.606 \\
0 \\
0.795\end{array}\right)$ & $\left(\begin{array}{c}0.548 \\
0 \\
0.837\end{array}\right)$ & $\left(\begin{array}{c}-0.837 \\
0 \\
0.548\end{array}\right)$ & 4.1 \\
\hline$\left(\begin{array}{l}0 \\
1 \\
1\end{array}\right)$ & $\left(\begin{array}{c}0 \\
0.559 \\
0.829\end{array}\right)$ & $\left(\begin{array}{c}0 \\
0.596 \\
0.803\end{array}\right)$ & $\left(\begin{array}{c}0.803 \\
0.596\end{array}\right)$ & 2.7 \\
\hline
\end{tabular}

When comparing the elastic constants in Table $6-5$, it is quite obvious that BMO exhibits a pronounced mechanical anisotropy. $c_{11}$, for example, characterizes the longitudinal compression along the $\boldsymbol{a}$-axis. It is considerably smaller than $c_{22}$ and $c_{33}$, which are the according quantities for the $\boldsymbol{b}$ - and $\boldsymbol{c}$-axis, respectively. Furthermore, the shear constant for the $\boldsymbol{b c}$-plane, $\boldsymbol{c}_{44}$, is much higher than the constants $c_{55}$ and $c_{66}$ that involve a component along the $\boldsymbol{a}$-direction. $c_{22}$ and $c_{33}$ as well as $c_{55}$ and $c_{66}$ agree within the experimental error. This unambiguously allows the conclusion that BMO is much softer along the $\boldsymbol{a}$-axis than in $\boldsymbol{b}$ - and $\boldsymbol{c}$-direction. This is also supported by the anisotropy of thermal expansion [95] and compressibility [98,99] at room temperature which are both stronger in a-direction.

This anisotropy can be explained by the crystal structure of $\mathrm{BMO}$, which is described in detail in chapter 2.3.3.1. In summary, the $\mathrm{Mn}^{4+}$-ions form rigid, infinite chains of edge-shared oxygen coordination octahedra. These chains are interconnected by dimers of pyramidally coordinated $\mathrm{Mn}^{3+}$-ions, but the connections are quite different along the $\boldsymbol{a}$ - and $\boldsymbol{b}$-direction, respectively, which is visualized in Figure 6-12. The basal planes of both pyramids share a common edge while all remaining corners are connected to the $\mathrm{Mn}^{4+}$-chains roughly along the $\boldsymbol{b}$-axis. This creates a rather rigid plane of coordinated oxygen atoms almost perpendicular to the $\boldsymbol{a}$-axis as indicated by a black dashed line in Figure 6-12. The apical oxygen atoms of both pyramids are pointing in opposite directions roughly along the $\boldsymbol{a}$-axis, which is marked by a green dashed line in Figure 6-12. This creates a rather lose connection in this direction and explains why BMO is softer along the $\boldsymbol{a}$-axis. 


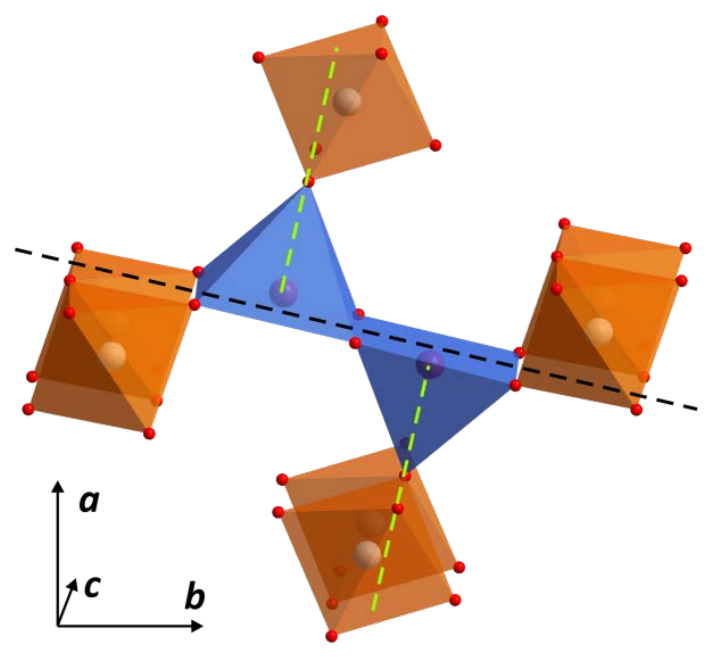

Figure 6-12: Visualization of the anisotropic interconnection of infinite $\mathrm{Mn}^{4+}$-octahedra chains (orange) by the $\mathrm{Mn}^{3+}$-pyramide dimers (blue). The rather rigid connection via the basal planes of the pyramids along the $\boldsymbol{b}$-axis is marked by a black dashed line. Green dashed lines are the weak connections in $\boldsymbol{a}$-direction along the apical oxygen atoms.

With knowledge of the elastic constants, it is possible to calculate the sound velocities of all three acoustic phonons for arbitrary propagation directions, which is depicted as a 3D-representations in Figure 6-13. A pronounced spatial anisotropy is observed for the transverse phonons, especially $T_{1} A$, which exhibits maxima of the sound velocity along the principal axes while distinct minima are found for the propagation directions in between.
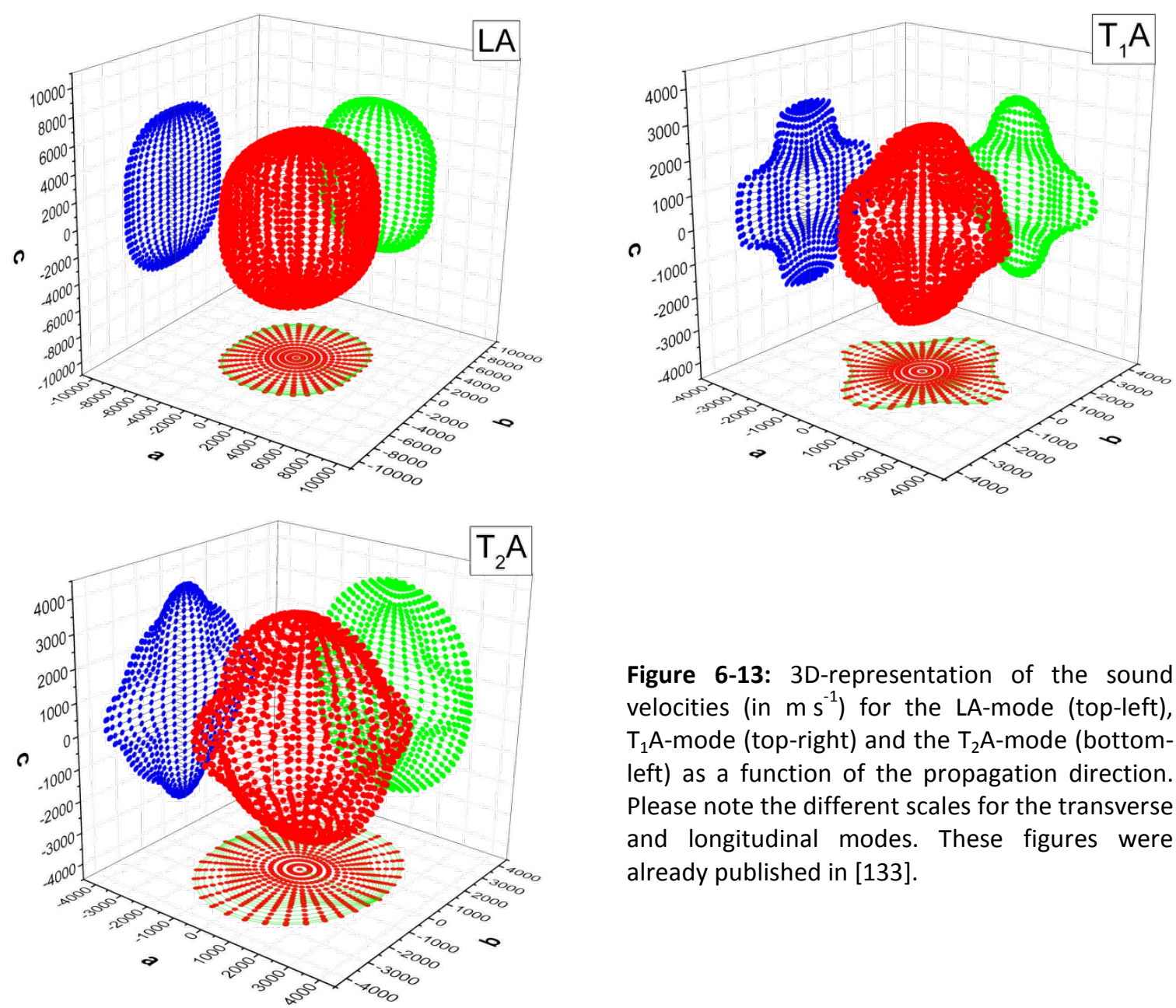

Figure 6-13: 3D-representation of the sound velocities (in $\mathrm{m} \mathrm{s}^{-1}$ ) for the LA-mode (top-left), $\mathrm{T}_{1} \mathrm{~A}$-mode (top-right) and the $\mathrm{T}_{2} \mathrm{~A}$-mode (bottomleft) as a function of the propagation direction. Please note the different scales for the transverse and longitudinal modes. These figures were already published in [133]. 


\subsubsection{Multiferroic phase transition}

The temperature-dependence of the elastic constants is subject of this chapter. Especially the behavior around and below $T_{\mathrm{N}}$ is of particular interest, because this is the temperature where multiferroicity starts to appear. This experiment is divided in two parts which are supposed to probe different effects that are expected to accompany the onset of ferroelectricity.

First, in analogy to the dielectric permittivity that exhibits a pronounced peak at the phase transition temperature, the elastic constants may also experience a sudden change at $T_{\mathrm{N}}$. This would manifest in an altered initial slope of the dispersion relation of certain acoustic phonons. In order to determine this change, one point of the dispersion of each relevant phonon was measured at a number of different temperatures in the vicinity of $T_{\mathrm{N}}$. Spectra of two transverse phonons are presented as arbitrary examples in Figure 6-14. Within the experimental accuracy, however, there are no significant differences between the individual spectra, which is also true for all other measured phonons. The same phonons of the room-temperature experiment were examined with the exception of LA with $\boldsymbol{q}=(\xi, \xi, 0)$. Its intensity is too low at this temperature to be reasonably measured during the limited time of the experiment. Nevertheless, since the dispersion of $T_{1} A$ with $\boldsymbol{q}=(\xi, \xi, 0)$ is sufficient to determine $c_{12}$, the lack of information about this particular pseudolongitudinal phonon does not restrict the general conclusion that none of the elastic constants exhibits any observable anomaly at the multiferroic phase transition temperature $T_{\mathrm{N}}$.

There are two main sources of error that restrict the significance of the result. First must be considered that the peak of the dielectric permittivity, which is presented in chapter 6.3.1, has a height of less than $3 \%$. Since the multiferroic coupling is not only the origin of the dielectric anomaly but also of a potential anomaly of the elastic constants, the latter might also be close to $3 \%$. The precision of the instrumental setup, however, only allows the determination of elastic constants with an error of $10 \%$. PUMA and IN8 might therefore not be precise enough to measure this anomaly if it
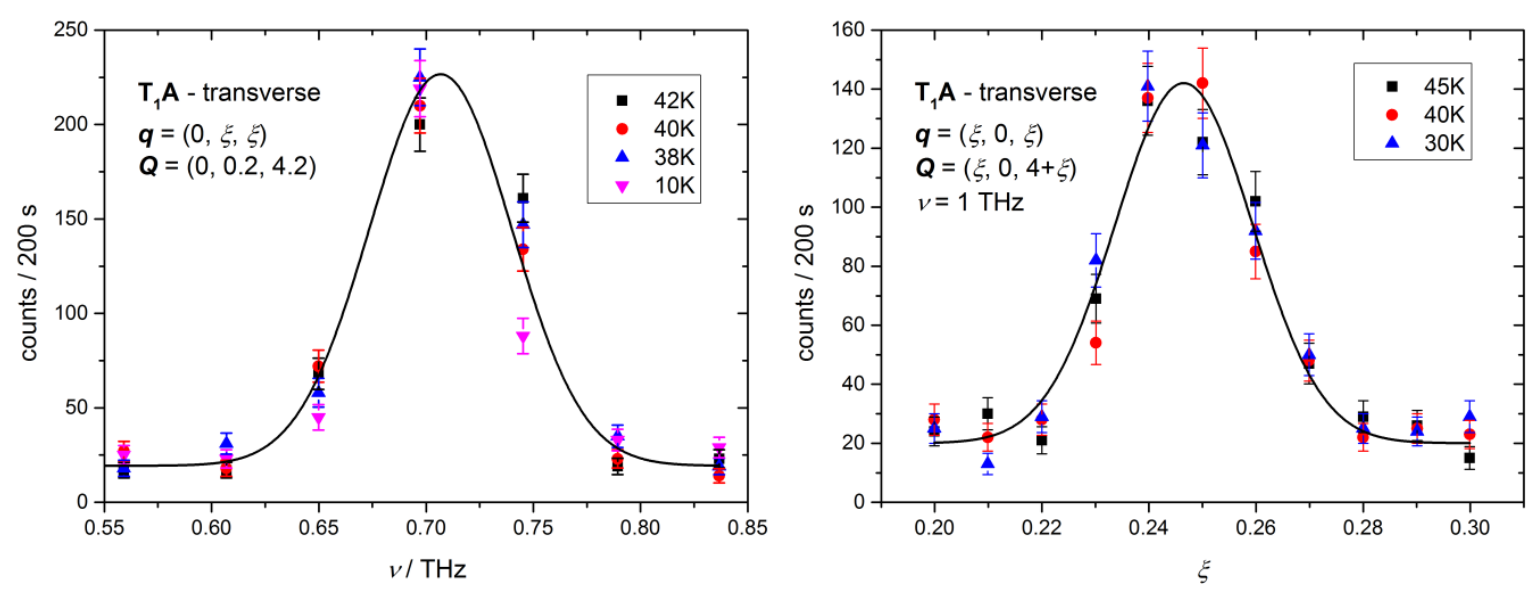

Figure 6-14: Comparison of spectra at temperatures in the vicinity of $T_{\mathrm{N}}$ of two exemplary transverse phonons. The black line is a Gauß-profile that was determined by a least-squares fit of the respective $40 \mathrm{~K}$ spectrum. 
exists at all. Another possible source of error is the accuracy of the temperature regulation. The dielectric anomaly occurs in a temperature range of less than $1 \mathrm{~K}$. Consequently, if the temperature regulation has a systematic error of only a few $0.1 \mathrm{~K}$, no effect can be observed.

In the second part of the experiment, the mechanical behavior of BMO at temperatures well below $T_{\mathrm{N}}$ is examined. The sublattice magnetization is an order parameter of the second order phase transition and therefore increases on cooling below $T_{\mathrm{N}}$ for several Kelvin until it reaches saturation. Consequently, any other property that is a product of the magnetization is also weak at $T_{\mathrm{N}}$ and increases with lower temperature. A good example is the variation of lattice parameter due to magnetostriction [105] which is proportional to the square of the sublattice magnetization. Since the spontaneous polarization in BMO might be induced by magnetostriction, according to Chapon, any structural change related to ferroelectricity and therefore any variation of the elastic constants should be stronger well below $T_{\mathrm{N}}$.

Consequently, the dispersion relation of all relevant phonons was measured at $30 \mathrm{~K}$ in order to determine the elastic constants. The same procedure of the room-temperature measurements is employed, but there are some restrictions to the data quality. First, the intensity of all phonons is significantly lower compared to room-temperature. This reduces the number of possible data points per dispersion curve, since only limited beam-time was available. Furthermore, the resolution of the focused monochromator at IN8 is lower compared to PUMA and in fact too low for the accurate measurement of phonon dispersions. Hence, all scans in the $\boldsymbol{a} \boldsymbol{b}$ - and $\boldsymbol{b} \boldsymbol{c}$-scattering plane were conducted with collimated beam, which increases the measurement time especially for transverse phonons. Therefore, only the positive direction of $\boldsymbol{q}$ was measured in most cases with the exception of same longitudinal phonons, where constant-energy-scans with low energy transfer also yield data points with $-\boldsymbol{q}$. In general, the determined sound velocities at low temperatures are less reliable than at room-temperature.

The dispersion relation of two exemplary phonons at $30 \mathrm{~K}$ and at room-temperature is presented in Figure 6-15. The data points of the transverse phonon in the left panel were collected in positive (closed symbols) and negative (open symbols) direction of $\boldsymbol{q}$ and corrected by an offset. There is no obvious temperature effect and the determined eigenvalue of the $30 \mathrm{~K}$ data falls into the $10 \%$ error margin of the room-temperature results.

This evaluation is not possible for the transverse phonon in the right panel. Since there are only three data points and none of them in - $\boldsymbol{q}$-direction, the eigenvalue has such a high uncertainty that it cannot be quantitatively evaluated. By looking at the dispersion curve, however, it is obvious that the slope is almost identical to room-temperature and the whole curve is only slightly shifted 
due to an offset, which can be explained by a small miscalibration of the instrument. It is therefore fair to assume that this phonon is not affected by the magnetic phase transition either, which is also true for all other phonons.

Since the eigenvalues of all phonons are either identical to the room-temperature results or could not accurately be determined, there will be no list of low-temperature elastic constants presented here. The results allow, however, the conclusion that any magnetoelastic effect, if present at all, is smaller than the $10 \%$ uncertainty of the experiment.
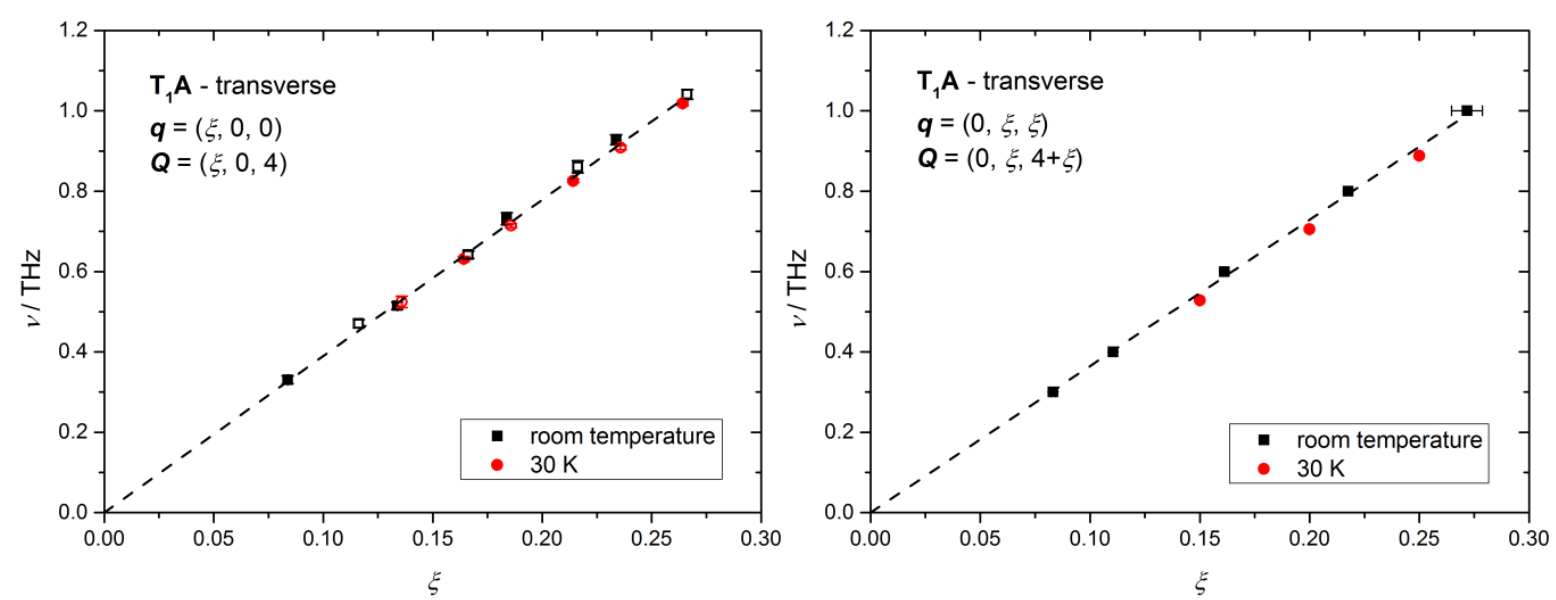

Figure 6-15: Comparison of the dispersion relation at room temperature (black symbols) and at $30 \mathrm{~K}$ (red symbols) for two exemplary transverse phonons. The data points that are represented with an open symbol in the left panel correspond to the dispersion curve in - $\boldsymbol{q}$-direction. The dispersion at $30 \mathrm{~K}$ in the left panel was measured at PUMA@FRMII while the data of the right panel were collected at IN8@ILL. 


\subsection{Conclusion}

The experiments on BMO during this thesis were intended to answer two questions: Is BMO multiferroic and if so, does the multiferroic coupling mechanism involve magnetoelastic interactions, as proposed by Chapon?

In order to answer the first question, the dielectric permittivity of BMO was measured in a range of temperatures above and below $T_{\mathrm{N}}$. There is a small but well defined peak-like anomaly in the real part of the permittivity which proves that BMO is indeed multiferroic. Ferroelectricity is only present along the $\boldsymbol{b}$-axis, which is in turn consistent with other members of the $\mathrm{R}_{2} \mathrm{Mn}_{4} \mathrm{O}_{10}$-family that also exhibit spontaneous polarization in $\boldsymbol{b}$-direction. The temperature of the ferroelectric phase transition is nearly identical to $T_{\mathrm{N}}$, which allows the conclusion that BMO is a type-II multiferroic. Both facts, the direction of ferroelectricity and the type-II nature of multiferroicity, are consistent with the multiferroic coupling mechanism that was suggested by Chapon.

Since Chapon's mechanism involves the displacement of Mn-atoms, the mechanical behavior of $\mathrm{BMO}$ is expected to change with the onset of magnetic order, if the mechanism is true. In order to give a concluding answer to this question, all elastic constants were determined by inelastic neutron scattering at room-temperature and in the vicinity of $T_{\mathrm{N}}$. BMO exhibits a pronounced mechanical anisotropy that can be explained on the basis of the crystal structure. There is, however, no observable temperature-dependent anomaly, neither directly at the multiferroic phase transition nor at $30 \mathrm{~K}$, well below $T_{\mathrm{N}}$. This is no unambiguous falsification of Chapon's theory though, because the experimental uncertainty of $10 \%$ is more than three times higher than the anomaly of the dielectric permittivity at $T_{\mathrm{N}}$, which has a relative height of less than $3 \%$. The precision of the instruments might simply be too low to detect the subtle influence of the magnetic order on the acoustic phonons. Nevertheless, it can be concluded that any magnetoelastic effect is smaller than $10 \%$, if present at all. 


\section{Summary}

The aim of this thesis is the characterization of three different multiferroic materials. Since each examined substance has its own issues that are currently subject of investigation, this thesis is divided in three distinct parts with different focus of research and different applied methods.

The first part is about $\mathrm{MnWO}_{4}$ whose magnetic phase transition temperatures can be increased when tungsten atoms are substituted by molybdenum. However, it is unclear have much molybdenum can be solved in $\mathrm{MnWO}_{4}$ and, consequently, how far the transition temperatures can be increased. This part of the thesis therefore aims at the determination of the miscibility gap and the maximum transition temperature. Two different ways of synthesis were tested, which mainly differ by the reaction temperature. It is possible to synthesize a metastable wolframite phase with up to $65 \%$ molybdenum on both ways, but the difference between both batches is the behavior during sintering at $1100^{\circ} \mathrm{C}$. The samples with a reaction temperature of $600^{\circ} \mathrm{C}$ decompose at higher temperatures, while the samples that were synthesized at $800^{\circ} \mathrm{C}$ remain stable. The metastable states of both batches are structurally identical, but they exhibit different magnetic properties. The $600^{\circ} \mathrm{C}$ samples are antiferromagnetically ordered like pure $\mathrm{MnWO}_{4}$, only the phase transition is shifted to higher temperatures. The $800^{\circ} \mathrm{C}$ sample with $50 \% \mathrm{Mo}$, on the other hand, exhibits a ferrimagnetic phase transition. However, a later attempt to reproduce this synthesis failed and a phase separation occurred during sintering, too. Therefore, the reaction temperature might not be the relevant synthesis parameter that promotes the stability of the Mo-rich wolframite phase. The decomposition of sintered tables for impedance spectroscopy indicates that the thermodynamic ground state at higher temperatures seems to be a miscibility gap with a lower limit of $x_{1}=0.3$.

In the second part of this thesis, magnon spectra were measured by inelastic neutron scattering in the multiferroic phase of $\mathrm{CuO}$ as well as in the paraelectric, antiferromagnetic phase for comparison. This research is motivated by the recent discovery of an electromagnon in the multiferroic phase, which can be regarded as an elementary excitation of the multiferroic coupling. Neutron polarization analysis allows an unambiguous assignment of all magnon eigenvectors, which are substantially different in both phases. In the paraelectric phase, all magnetic excitations correspond to a displacement of spins perpendicular to the $\boldsymbol{b}$-axis with an anisotropic spin-gap that depends on the displacement direction. In the AF2-phase, on the other hand, two distinct excitations are identified. The first one is a phason mode where the spins rotate inside the cycloidal plane and that has no spin-gap within the instrumental resolution. The second mode might be the electromagnon, because the determined eigenvector matches the predicted displacement pattern, which is a rotation of the whole cycloidal plane. Furthermore, the energy of the electromagnon at the magnetic $\Gamma$-point was determined to be $3 \mathrm{meV}$, which is exactly the value of the spin-gap of this 
second mode. Both observations are clear indicators that this mode is indeed the electromagnon, but it is no conclusive evidence, since there is no way to distinguish common magnons from electromagnons with inelastic neutron scattering.

The aim of the third part of this thesis is to answer the question whether $\mathrm{Bi}_{2} \mathrm{Mn}_{4} \mathrm{O}_{10}$ is multiferroic, because there is no experimental evidence for ferroelectricity in the antiferromagnetic phase, unlike other members of the $\mathrm{R}_{2} \mathrm{Mn}_{4} \mathrm{O}_{10}$-family. Therefore, the dielectric permittivity of $\mathrm{Bi}_{2} \mathrm{Mn}_{4} \mathrm{O}_{10}$ was measured in a range of temperatures above and below $T_{\mathrm{N}}$. There is a small but well defined peak-like anomaly in the real part of the permittivity along the $\boldsymbol{b}$-axis, which is evidence for the onset of ferroelectricity. The temperature of this phase transition is nearly identical to $T_{\mathrm{N}}$, which allows the conclusion that $\mathrm{Bi}_{2} \mathrm{Mn}_{4} \mathrm{O}_{10}$ is a type-II multiferroic. The multiferroic coupling mechanism that is proposed to induce ferroelectricity in $\mathrm{R}_{2} \mathrm{Mn}_{4} \mathrm{O}_{10}$ involves the displacement of $\mathrm{Mn}$-atoms. The mechanical behavior of $\mathrm{Bi}_{2} \mathrm{Mn}_{4} \mathrm{O}_{10}$ is therefore expected to change with the onset of magnetic order. In order to answer the question if this mechanism can also be applied to $\mathrm{Bi}_{2} \mathrm{Mn}_{4} \mathrm{O}_{10}$, all elastic constants were determined in the multiferroic phase as well as at room temperature for comparison. $\mathrm{Bi}_{2} \mathrm{Mn}_{4} \mathrm{O}_{10}$ exhibits a pronounced mechanical anisotropy that can be explained on the basis of the crystal structure. There is, however, no observable temperature-dependent anomaly, neither directly at the multiferroic phase transition nor well below $T_{\mathrm{N}}$. Any magnetoelastic effect, if present at all, must therefore be smaller than the $10 \%$ instrumental accuracy. 


\section{References}

[1] Maxwell, J. C., A dynamical theory of the electromagnetic field, Phil. Trans. Roy. Soc. London $1865,155,459-512$.

[2] Astrov, D. N., The magnetoelectric effect in antiferromagnetics, Soviet Phys. JETP 1960, 11, 708-709.

[3] Eerenstein, W.; Mathur, N.; Scott, J. F., Multiferroic and Magnetoelectric Materials, Nature 2006, 442, 759-756.

[4] Smolenskii, G.; Chupis, I. E., Ferroelectromagnets, Sov. Phys. Usp. 1982, 25, 475-493.

[5] Ascher, E. et al., Some Properties of Ferromagnetoelectric Nickel - Iodine Boracite, Ni3B7013I, J. Appl. Phys. 1966, 37, 1404-1405.

[6] Schmid, H., Multi-ferroic Magnetoelectrics, Ferroelectrics 1994, 162, 317-338.

[7] Aizu, K., Possible Species of Ferromagnetic, Ferroelectric and Ferroelastic Crystals, Phys. Rev. B $1970,2,754-772$.

[8] Hill, N., Why are there so few magnetic ferroelectrics, J. Phys. Chem. B 2000, 104, 6694-6709.

[9] Khomskii, D. I., Multiferroics, J. Magn. Magn. Mater. 2006, 306, 1-8.

[10] Khomskii, D. I., Classifying multiferroics, Physics 2009, 2, 628.

[11] Kimura, T. et al., Magnetic control of ferroelectric polarization, Nature 2003, 426, 55-58.

[12] Mostovoy, M., Ferroelectricity in Spiral Magnets, Phys. Rev. Lett. 2006, 96, 676011.

[13] Katsura, H.; Nagaosa, N.; Balatsky, A. V., Spin current and magnetoelectric effect in noncollinear magnets, Phys. Rev. B 2005, 95, 57205.

[14] Sergienko, I. A.; Dagotto, E., Role of the Dzyaloshinskii-Moriya interaction in multiferroic perovskites, Phys. Rev. B 2006, 73, 94434.

[15] Taniguchi, K. et al., Magnetic-field dependence of the ferroelectric polarization and spin-lattice coupling in multiferroic MnWO4, Phys. Rev. B 2008, 77, 64408.

[16] Fiebig, M., Revival of the magnetoelectric effect, J. Phys. D: Appl. Phys. 2005, 38, R123-R152.

[17] Zhao, L. et al., Oxyhalides: A new class of high-T C multiferroic materials, Sci. Adv. 2016, 2, e1600353.

[18] Meddar, L. et al., Increasing the Phase-Transition Temperatures in Spin-Frustrated Multiferroic MnWO4 by Mo Doping, Chem. Mater. 2012, 24, 353-360.

[19] Kimura, T. et al., Cupric oxide as an induced-multiferroic with high-TC, Nat. Mater. 2008, 7, 291-294.

[20] Jones, S. P. P. et al., High-temperature electromagnons in the magnetically induced multiferroic cupric oxide driven by intersublattice exchange, Nat. Commun. 2014, 5, 3787.

[21] Chapon, L. C. et al., Structural anomalies and multiferroic behavior in magnetically frustrated TbMn2O5, Phys. Rev. Lett. 2004, 93, 177402. 
[22] Spaldin, N. A.; Fiebig, M.; Mostovoy, M., The toroidal moment in condensed-matter physics and its relation to the magnetoelectric effect, J. Phys.: Condens. Matter 2008, 20, 434203.

[23] Schmid, H., Some symmetry aspects of ferroics and single phase multiferroics, J. Phys.: Condens. Matter 2008, 20, 434201.

[24] Litvin, D. B., Ferroic classifications extended to ferrotoroidic crystals, Acta Cryst. A 2008, 64, $316-320$.

[25] Ascher, E., Some Properties of Spontaneous Currents, Helv. Phys. Acta 1966, 39, 40-48.

[26] Anderson, P. W., New Approach to the Theory of Superexchange Interactions, Phys. Rev. 1959, $115,2-13$.

[27] Cheong, S. W.; Mostovoy, M., Multiferroics: a magnetic twist for ferroelectricity, Nat. Mater. $2007,6,13-20$.

[28] Tolédano, P. et al., Primary ferrotoroidicity in antiferromagnets, Phys. Rev. B 2015, 92, 1420.

[29] van den Brink, J.; Khomskii, D. I., Multiferroicity due to charge ordering, J. Phys.: Condens. Matter 2008, 20, 434217.

[30] Moriya, T., Anisotropic Superexchange Interaction and Weak Ferromagnetism, Phys. Rev. $1960,120,91-98$.

[31] Moriya, T., New Mechanism of Anisotropic Superexchange Interaction, Phys. Rev. Lett. 1960, 4, $228-230$.

[32] Ye, F. et al., Long-range magnetic interactions in the multiferroic antiferromagnet MnWO4, Phys. Rev. B 2011, 83, 140401.

[33] Dzyaloshinsky, I., A thermodynamic theory of weak ferromagnetism of antiferromagnets, $J$. Phys. Chem. Solids 1958, 4, 241-255.

[34] Tokura, Y.; Seki, S., Multiferroics With Spiral Spin Orders, Adv. Mater. 2010, 22, 1554-1565.

[35] Arkenbout, A. et al., Ferroelectricity in the cycloidal spiral magnetic phase of MnWO4, Phys. Rev. B 2006, 74, 184431.

[36] Volkova, L. M.; Marinin, D. V., Crystal chemistry aspects of the magnetically induced ferroelectricity in TbMn2O5 and BiMn2O5, J. Phys.: Condens. Matter 2009, 21, 15903.

[37] Dachs, H.; Stoll, E.; Weitzel, H., Kristallstruktur und magnetische Ordnung des Hübnerits, MnWO4, Z. Krist. 1967, 125, 120-129.

[38] Lautenschläger, G. et al., Magnetic phase transitions of MnWO4 studied by the use of neutron diffraction, Phys. Rev. B 1993, 48, 6087-6098.

[39] Macavei, J.; Schulz, H., The Crystal Structure of Wolframite Type Tungstates at High Pressure, Z. Krist. 1993, 207, 193-208. 
[40] Landee, C. P.; Westrum, E. F., Thermophysical measurements on transition-metal tungstates III. Heat capacity of antiferromagnetic manganese tungstate, J. Chem. Thermodyn. 1976, 8, 663-674.

[41] Hollmann, N. et al., Local symmetry and magnetic anisotropy in multiferroic MnWO4 and antiferromagnetic CoWO4 studied by soft x-ray absorption spectroscopy, Phys. Rev. B 2010, $82,184429$.

[42] Ehrenberg, H. et al., Magnetic Phase Diagrams of MnWO4, J. Phys.: Condens. Matter 1997, 9, 3189-2303.

[43] Urcelay-Olabarria, I. et al., Incommensurate magnetic structures of multiferroic MnWO4 studied within the superspace formalism, Phys. Rev. B 2013, 87, 14419.

[44] Sagayama, H. et al., Correlation between ferroelectric polarization and sense of helical spin order in multiferroic MnWO4, Phys. Rev. B 2008, 77, 220407.

[45] Ehrenberg, H. et al., Magnon dispersion in MnWO4, J. Phys.: Condens. Matter 1999, 11, 26492659.

[46] Xiao, Y. et al., Spin-wave and electromagnon dispersions in multiferroic MnWO4 as observed by neutron spectroscopy, Phys. Rev. B 2016, 93, 214428.

[47] Taniguchi, K. et al., Ferroelectric Polarization Flop in a Frustrated Magnet MnWO4 Induced by a Magnetic Field, Phys. Rev. Lett. 2006, 97, 97203.

[48] Chaudhury, R. P. et al., Thermal expansion and pressure effect in MnWO4, Physica B 2008, $403,1428-1430$.

[49] Kundys, B.; Simon, C.; Martin, C. Y., Effect of magnetic field and temperature on the ferroelectric loop in MnWO4, Phys. Rev. B 2008, 80, 212302.

[50] Taniguchi, K. et al., Magnetoelectric Memory Effect in the Nonpolar Phase with Collinear Spin Structure in Multiferroic MnWO4, Phys. Rev. Lett. 2009, 102, 147201.

[51] Meier, D. et al., Topology and manipulation of multiferroic hybrid domains in MnWO4, Phys. Rev. B 2009, 80, 224420.

[52] Finger, T. et al., Electric field control of the chiral magnetism of multiferroic MnWO4 as seen via polarized neutron diffraction, Phys. Rev. B 2010, 81, 54430.

[53] Baum, M. et al., Kinetics of the multiferroic switching in MnWO4, Phys. Rev. B 2014, 89, 144406.

[54] Mitamura, H. et al., Sign reversal of the dielectric polarization of MnWO4 in very high magnetic fields, J. Phys.: Conf. Ser. 2009, 150, 42126.

[55] Felea, V. et al., Magnetic Phase Diagram of Multiferroic MnWO4 Probed by Ultrasound, J. Phys.: Condens. Matter 2011, 23, 216001.

[56] Urcelay-Olabarria, I. et al., X phase of MnWO4, Phys. Rev. B 2014, 90, 24408. 
[57] Urcelay-Olabarria, I.; García-Muñoz, J. L.; Mukhin, A. A., Field effects with H // b on the incommensurate magnetic structures of multiferroic MnWO4 studied within the superspace formalism, Phys. Rev. B 2015, 91, 104429.

[58] Kumar, C. M. N. et al., Crystal structure, incommensurate magnetic order, and ferroelectricity in Mn1-xCuxWO4 (0 $\leq x \leq 0.19$ ), Phys. Rev. B 2015, 91, 127.

[59] Song, Y.-S. et al., Observation of spontaneous ferroelectric polarization reversal in multiferroic Mn1-xNixWO4 (x 0.16), Appl. Phys. Lett. 2014, 104, 252904.

[60] Yang, J. et al., Modulated multiferroic properties of MnWO 4 via chemical doping, RSC Adv. 2016, 6, 3219-3223.

[61] Song, Y.-S. et al., Stabilization of the elliptical spiral phase and the spin-flop transition in multiferroic Mn1-xCoxWO4, Phys. Rev. B 2009, 79, 224415.

[62] Meddar, L. et al., Effect of Nonmagnetic Substituents Mg and $\mathrm{Zn}$ on the Phase Competition in the Multiferroic Antiferromagnet MnWO 4, Chem. Mater. 2009, 21, 5203-5214.

[63] Hardy, V. et al., Phase transitions and magnetic structures in MnW1-xMoxO4 compounds, J. Phys. Condens. Matter 2016, 28, 336003.

[64] Fang, Y. et al., Modulation of magnetic properties and enhanced magnetoelectric effects in MnW1-xMoxO4 compounds, Chinese Phys. B 2014, 23, 77502.

[65] Allred, A. L., Electronegativity values from thermochemical data, J. Inorg. Nucl. Chem 1961, 17, $215-221$.

[66] Whittaker, E. J. W.; Muntus, R., Ionic radii for use in geochemistry, Geochim. Cosmochim. Acta 1970, 34, 945-956.

[67] Shannon, R. D., Revised effective ionic radii and systematic studies of interatomic distances in halides and chalcogenides, Acta Cryst. A 1976, 32, 751-767.

[68] Abrahams, S. C.; Reddy, J. M., Crystal Structure of the Transition-Metal Molybdates. I. Paramagnetic Alpha-MnMoO4, J. Chem. Phys. 1965, 43, 2533.

[69] Chang, L. Y., Subsolidus phase relations in the system ZnWO4-ZnMoO4-MnWO4-MnMoO4, Mineral. Mag. 1968, 36, 992-996.

[70] Chang, L. Y., Solid Solutions of Scheelite with other RIIWO4-Type Tungstates, Am. Mineral. $1967,52,427-435$.

[71] Blanco-Gutierrez, V. et al., Phase transitions in $\mathrm{Mn}(\mathrm{Mo1}-\mathrm{xWx}) \mathrm{O} 4$ oxides under the effect of high pressure and temperature, phys. stat. sol. (b), 253, 2043-2048.

[72] Asbrink, S.; Norrby, L.-J., A refinement of the crystal structure of copper(II) oxide with a discussion of some exceptional e.s.d.'s, Acta Cryst. B 1970, 26, 8-15.

[73] Asbrink, S.; Waskowska, A., CuO: X-ray single-crystal structure determination at $196 \mathrm{~K}$ and room temperature, J. Phys.: Condens. Matter 1991, 3, 8173-8180. 
[74] Brockhouse, B. N., Antiferromagnetism in Cuprix Oxide, Phys. Rev. 1954, 94, 781.

[75] Forsyth, J. B.; Brown, P. J.; Wanklyn, B. M., Magnetism in cupric oxide, J. Phys. C: Solid State Phys. 1988, 21, 2917-2929.

[76] Brown, P. J. et al., Antiferromagnetism in CuO studied by neutron polarimetry, J. Phys.: Condens. Matter 1991, 3, 4281-4287.

[77] Ain, M. et al., Magnetic structure of $\mathrm{CuO}$ by neutron diffraction with polarization analysis, $J$. Phys.: Condens. Matter 1992, 4, 5327-5338.

[78] Yang, B. X.; Tranquada, J. M.; Shirane, G., Neutron scattering studies of the magnetic structure of cupric oxide, Phys. Rev. B 1988, 38, 174-178.

[79] Zheng, X.-G. et al., Evidence of Charge Stripes, Charge-Spin-Orbital Coupling and Phase Transition in a Simple Copper Oxide CuO, J. Phys. Soc. Jpn. 2001, 70, 1054-1063.

[80] Ohashi, M. et al., Effect of pressure on the magnetic phase transition in cupric oxide, Phys. Rev. B 2006, 73, 134421.

[81] Wang, F. et al., Persistent multiferroicity without magnetoelectric effects in CuO, J. Appl. Phys. 2011, 110, 54106.

[82] Villarreal, R. et al., Magnetic Phase Diagram of CuO via High-Resolution Ultrasonic Velocity Measurements, Phys. Rev. Lett. 2012, 109, 167206.

[83] Rebello, A. et al., Multiple phase transitions in CuO observed with thermal expansion, Phys. Rev. B 2013, 88.

[84] Chattopadhyay, T. et al., Anisotropic spin correlations in CuO above the Néel temperature, Physica C 1990, 170, 371-374.

[85] O'Keeffe, M.; Stone, F. S., The magnetic susceptibility of cupric oxide, J. Phys. Chem. Solids $1962,23,261-266$.

[86] Ain, M. et al., Magnetic excitations in CuO, Physica C 1989, 162-164, 1279-1280.

[87] Boothroyd, A. T. et al., High-energy magnetic excitations in CuO, Physica B 1997, 234-236, 731-733.

[88] Jacobsen, $\mathrm{H}$. et al., Spin dynamics and exchange interactions in $\mathrm{CuO}$ measured by neutron scattering, Phys. Rev. B 2018, 97, 1224.

[89] Yang, B. X. et al., Magnetic neutron scattering study of single-crystal cupric oxide, Phys. Rev. B 1989, 39, 4343-4349.

[90] Chattopadhyay, T. et al., Magnetic excitations and spin correlations in CuO, Physica B 1992, 180-181, 420-422.

[91] Babkevich, P. et al., Electric field control of chiral magnetic domains in the high-temperature multiferroic CuO, Phys. Rev. B 2012, 85, 134428. 
[92] Wang, Z. et al., Magnetoelectric effect and phase transitions in $\mathrm{CuO}$ in external magnetic fields, Nat. Commun. 2016, 7, 10295.

[93] Bertaut, E. F. et al., Structure magnetique et proprietes magnetiques de BiMn2O5, Solid State Commun. 1967, 5, 25-30.

[94] Niizeki, N.; Wachi, M., The crystal structures of Bi2Mn4O10, Bi2Al4O9 and Bi2Fe4O9, Z. Krist. $1968,127,173-187$.

[95] Burianek, M. et al., Single crystal growth and characterization of mullite-type Bi2Mn4O10, Int. J. Mat. Res. 2012, 103, 449-455.

[96] Nguyen, N. et al., Distribution of $\mathrm{Mn3}+$ and Mn4+ species between octahedral and square pyramidal sites in Bi2Mn4O10-type structure, J. Mater. Chem. 1999, 9, 731.

[97] Muñoz, A.; Fernández-Díaz, M. T., Magnetic structure and properties of BiMn2O5 oxide: A neutron diffraction study, Phys. Rev. B 2002, 65, 144423.

[98] Lopez-de-la-torre, L. et al., High-pressure behavior of the ternary bismuth oxides Bi2Al4O9Bi2Al4O9, Bi2Ga4O9Bi2Ga4O9 and Bi2Mn4O10, J. Sol. State Chem. 2009, 182, 767777.

[99] Grzechnik, A. et al., High-pressure behaviours of HoMn2O5 and BiMn2O5, J. Phys.: Condens. Matter 2010, 22, 275401.

[100] Curti, M. et al., Liebau density vector, Z. Krist. 2013, 228, 629.

[101] Liu, Q. et al., On the Anomalous Magnetic Behavior and the Multiferroic Properties in BiMn2O5, Inorg. Chem. 2013, 52, 7853-7861.

[102] Radaelli, P. G.; Chapon, L. C., A neutron diffraction study of RMn2O5 multiferroics, J. Phys.: Condens. Matter 2008, 20, 434213.

[103] Vecchini, C. et al., Commensurate magnetic structures of $\mathrm{RMn} 2 \mathrm{O} 5(\mathrm{R}=\mathrm{Y}, \mathrm{Ho}, \mathrm{Bi})$ determined by single-crystal neutron diffraction, Phys. Rev. B 2008, 77, 134434.

[104] Chapon, L. C. et al., Ferroelectricity induced by acentric spin-density waves in YMn2O5, Phys. Rev. Lett. 2006, 96, 97601.

[105] Granado, E. et al., Magnetoelastic and thermal effects in the BiMn2O5 lattice: A highresolution x-ray diffraction study, Phys. Rev. B 2008, 77, 134101.

[106] García-Flores, A. F. et al., Anomalous phonon shifts in the paramagnetic phase of multiferroic $\mathrm{RMn2O5}$ ( $\mathrm{R}=\mathrm{Bi}, \mathrm{Eu}, \mathrm{Dy})$ : Possible manifestations of unconventional magnetic correlations, Phys. Rev. B 2006, 73, 104411.

[107] Ramirez, F. et al., Magnetic and transport properties assisted by local distortions in Bi2Mn4O10 and Bi2Fe4O9 multiferroic compounds, J. Alloys Compd. 2015, 651, 405-413.

[108] Golovenchits, E. I., Magnetic and structural correlations in EuMnO and BiMnO crystals in the paramagnetic temperature range, J. Exp. Theor. Phys. 1997, 85, 156-162. 
[109] Fier, I. et al., Contribution of an extrinsic mechanism for the electrical polarization in BiMn2O5 ceramics, AIP Advances 2012, 2, 42165.

[110] Yin, L. H. et al., Dielectric relaxations and magnetodielectric response in BiMn2O5 single crystal, Appl. Phys. Lett. 2013, 103, 152908.

[111] Noda, Y. et al., Magnetic and ferroelectric properties of multiferroic RMn2O5, J. Phys.: Condens. Matter 2008, 20, 434206.

[112] Sobolev, O.; Park, J. T., PUMA, JLSRF 2015, 1, A13.

[113] Moon, R. M.; Riste, T.; Koehler, W. C., Polarization analysis of thermal-neutron scattering, Phys. Rev. 1969, 181, 920-931.

[114] Schwesig, S. et al., Novel type of neutron polarization analysis using the multianalyzerequipment of the three-axes spectrometer PUMA, Nucl. Instrum. Methods Phys. Res. A 2018, $877,124-130$.

[115] Cole, K. S.; Cole, R. H., Dispersion and Absorption in Dielectrics I. Alternating Current Characteristics, J. Chem. Phys. 1941, 9, 341-351.

[116] Toby, B. H.; Dreele, R. B. von, GSAS-II, J Appl Crystallogr 2013, 46, 544-549.

[117] Iliev, M. N.; Gospodinov, M. M.; Litvinchuk, A. P., Raman spectroscopy of MnWO4, Phys. Rev. B 2009, 80, 212302.

[118] Hoang, L. et al., Temperature-dependent Raman scattering study of multiferroic MnWO4, J. Raman Spectrosc. 2010, 41, 1005-1010.

[119] Ziegler, F. Ramanspektroskopie an multiferroischem Manganwolframat, Masterarbeit, GeorgAugust Universität, Göttingen, 2014.

[120] Young, A. P.; Schwartz, C. M., High-Pressure Synthesis of Molybdates with the Woiframite Structure, Science 1963, 141, 348-349.

[121] Ehrenberg, H.; Schwarz, B.; Weitzel, H., Magnetic phase diagrams of -MnMoO4, J. Magn. Magn. Mater. 2006, 305, 57-62.

[122] Katsura, H.; Balatsky, A. V.; Nagaosa, N., Dynamical magnetoelectric coupling in helical magnets, Phys. Rev. Lett. 2007, 98, 1-4.

[123] Senff, D. et al., Magnetic excitations in a cycloidal magnet: the magnon spectrum of multiferroic TbMnO3, J. Phys.: Condens. Matter 2008, 20, 434212.

[124] Cao, K.; Giustino, F.; Radaelli, P. G., Theory of Electromagnons in CuO, Phys. Rev. Lett. 2015, 114, 197201.

[125] Eckold, G. et al., Temperature-dependent investigation of the mechanical anisotropy of Bi2Mn4O10 in the multiferroic phase, Institut Laue-Langevin (ILL), 2017.

[126] Fier, I.; Walmsley, L.; Souza, J. A., Relaxor behavior in multiferroic BiMn2O5 ceramics, J. Appl. Phys. 2011, 110, 84101. 
[127] Sun, Z. H. et al., Effect of Ce substitution on magnetic and dielectric properties of BiMn2O5, J. Appl. Phys. 2006, 99, 084105.

[128] Lin, Y. Q. et al., Dielectric relaxation mechanisms of BiMn[sub 2]O[sub 5] ceramics, J. Appl. Phys. 2009, 105, 54109.

[129] Lin, Y. Q.; Chen, X. M., Dielectric relaxation and polaronic conduction in double perovskite La2MgMnO6, Appl. Phys. Lett. 2010, 96, 142902.

[130] Ang, C.; Yu, Z.; Cross, L. E., Oxygen-vacancy-related low-frequency dielectric relaxation and electrical conduction in Bi, Phys. Rev. B 2000, 62, 228-236.

[131] Khannanov, B. et al., Electric polarization induced by phase separation in magnetically ordered and paramagnetic states of RMn2O5 (R=Gd, Bi), J. Magn. Magn. Mater. 2017, 421, 326-335.

[132] Eckold, G.; Stein-Arsic, M.; Weber, H. J., UNISOFT - a program package for lattice-dynamical calculations, J App/ Crystallogr 1987, 20, 134-139.

[133] Ziegler, F. et al., Mechanical properties of multiferroic Bi2Mn4O10: Full set of elastic constants determined by inelastic neutron scattering, phys. stat. sol. (b) 2016, 253, 976-982. 


\section{Danksagung}

Abschließend möchte ich all jenen danken, die mir in den letzten Jahren geholfen haben, diese Arbeit anzufertigen.

Ganz besonders möchte ich mich bei Herrn Prof. Dr. Eckold bedanken, der mir mit der Aufnahme in seinen Arbeitskreis die vorliegende Arbeit erst ermöglichte. Bei allen Schritten stand er mir mit Rat zur Seite. Insbesondere für die Möglichkeit Forschung an Großgeräten wie PUMA durchzuführen bin ich ihm dankbar.

Herrn Prof. Dr. Stalke gilt mein Dank dafür, dass er Zweitbetreuer meiner Arbeit geworden ist. Weiterhin möchte ich mich bei allen Mitgliedern des Prüfungsausschusses bedanken.

Bei Prof. Dr. Gesing und Herrn Dr. Murshed möchte ich mich für die Kooperation bedanken, die die Messungen an $\mathrm{Bi}_{2} \mathrm{Mn}_{4} \mathrm{O}_{10}$ überhaupt erst ermöglicht hat. I would like to thank Dr. Piovano and Dr. Ivanov for the support during the experiment at IN8@ILL. Herrn Dr. Demeshko gilt mein Dank für die Magnetisierungsmessungen an Mo-dotiertem $\mathrm{MnWO}_{4}$.

Außerdem möchte ich mich bei allen jetzigen und ehemaligen Mitgliedern der Abteilung Physikalische Chemie fester Körper bedanken. Die vielen angeregten Diskussion sowie die gelegentlichen, gemeinsamen Unternehmungen haben eine angenehme Arbeitsatmosphäre geschaffen.

Mein Dank gilt dabei Holger Gibhardt für die gemeinsamen Messreisen nach Garching und Grenoble, für Unterstützung bei den Ramanmessungen und vieles mehr. Ohne Karsten Behrendt wären die Impedanzmessungen niemals möglich gewesen, wofür ich ihm dankbar bin. Jeannis Leist möchte ich für die technische Hilfe und Anleitung bei Probenpräparation und instrumentellem Aufbau danken. Friedrich Güthoff gilt mein Dank für seine Unterstützung bei der Röntgendiffraktion. Bei Patrick Kirscht möchte ich mich dafür bedanken, dass er jederzeit als Ansprechpartner bei unterschiedlichen, technischen Problemstellungen bereit stand. Genauso gilt mein Dank Olaf Wenzel für die vielfältige, technische Hilfe, die er geleistet hat. Steffen Schwesig bin ich für seine Zusammenarbeit bei allen Messreisen im Zusammenhang mit der Polarisationsanalyse dankbar.

Norbert Jünke gilt mein Dank für die technische Unterstützung am PUMA. Genauso möchte ich Oleg Sobolev für seine geduldige Hilfe bei den Messreisen am PUMA danken. Furthermore, I would like to thank Avishek Maity for his support on the polarization analysis setup.

Außerdem möchte ich den Bachelor- und MasterstudentInnen Lennart Köhler, Leonie Gomell und Zibo Zhao für ihren Beitrag zu dieser Arbeit danken. 
Abschließend möchte ich mich bei meinen Eltern bedanken, die mir mein Studium erst ermöglichten. 